\title{
Iridium(III)-catalyzed C(3)-H Alkylation of Isoquinolines via Metal Carbene Migratory Insertion
}

Neha Jha, Roushan Prakash Singh, Paridhi Saxena and Manmohan Kapur*

Department of Chemistry, Indian Institute of Science Education and Research Bhopal, Bhopal

Bypass Road, Bhauri, Bhopal 462066, Madhya Pradesh, India.

E-mail: mk@iiserb.ac.in

\section{Table of contents}

$\begin{array}{ll}\text { General Methods } & \text { S2 }\end{array}$

General procedures and analytical data of starting materials $\quad$ S2 - S14

$\begin{array}{ll}\text { Optimization tables } & \text { S14 - S17 }\end{array}$

General procedure for Ir(III)-catalyzed C(3)- alkylation and analytical data $\quad$ S17 - S27

$\begin{array}{lr}\text { Aromatization and synthetic applications } & \text { S27 - S32 }\end{array}$

$\begin{array}{lr}\text { Control experiments and mechanistic studies } & \text { S32 - S37 }\end{array}$

$\begin{array}{ll}\text { References } & \text { S37 }\end{array}$

${ }^{1} \mathrm{H}-,{ }^{13} \mathrm{C}-\mathrm{NMR}$ spectra of compounds $\quad$ S38 - S96

$\begin{array}{ll}\text { Variable Temperature NMR } & \text { S97 - S98 }\end{array}$

$\begin{array}{lr}\text { Mass Experiments } & \text { S99 }-\mathrm{S} 100\end{array}$

$\begin{array}{ll}\text { X-ray diffraction data } & \text { S101 - S103 }\end{array}$ 


\section{EXPERIMENTAL SECTION:}

\section{(i) General Methods:}

All commercially available compounds were used without purification. Unless otherwise noted, all reactions were performed in oven-dried glassware. All reactions were run under nitrogen (oxygen atmosphere only if indicated). All solvents used in the reactions were purified before use. Tetrahydrofuran and toluene were distilled from sodium and benzophenone; 1,1,1,3,3,3hexafluoroisopropanol (HFIP) was distilled and stored over $3 \AA$ molecular sieves. Petroleum ether with a boiling range of $40-60{ }^{\circ} \mathrm{C}$ was used. Melting points are uncorrected. ${ }^{1} \mathrm{H}-$ and ${ }^{13} \mathrm{C}$ NMR: Recorded on 400, 500 and $700 \mathrm{MHz}$ NMR Spectrometers; spectra were recorded at $295 \mathrm{~K}$ in $\mathrm{CDCl}_{3}$; chemical shifts are calibrated to the residual proton and carbon resonance of the solvent: $\mathrm{CDCl}_{3}\left({ }^{1} \mathrm{H} \delta 7.26 ;{ }^{13} \mathrm{C} \delta\right.$ 77.0). LC-HRMS: Recorded on a Q-ToF with Electron Spray Ionization (ESI) or Atmospheric Pressure Chemical Ionization (APCI). GC-LRMS: Performed GC-MS (EI $70 \mathrm{eV}$ ) using DB-5 column. Single-crystal X-ray diffraction data were collected using a Bruker SMART APEX II CCD diffractometer with graphite monochromated Mo K $\alpha(\lambda=$ $0.71073 \AA$ ) radiation at low temperatures.

\section{(ii) General Procedures and analytical data of starting materials}

Scheme S1: Synthesis of 5-bromoisoquinoline (1n): ${ }^{1}$

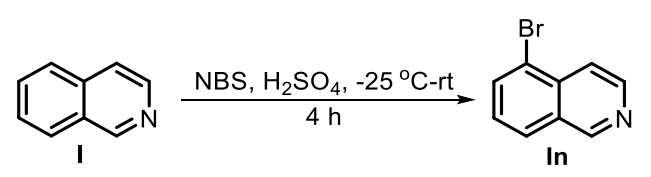

To the stirring solution of concentrated $\mathrm{H}_{2} \mathrm{SO}_{4}(10 \mathrm{ml})$ at $0{ }^{\circ} \mathrm{C}$, isoquinoline $\mathbf{I}(1 \mathrm{~g}, 7.75 \mathrm{mmol})$ was added slowly. The resulting mixture was cooled to $-25^{\circ} \mathrm{C}$ followed by the addition of $\mathrm{N}$ bromo succinimide $(1.37 \mathrm{~g}, 7.75 \mathrm{mmol})$, in portions, over a period of $1.5 \mathrm{~h}$ and the temperature was maintained between $-25{ }^{\circ} \mathrm{C}$ to $-20{ }^{\circ} \mathrm{C}$. The reaction mixture was stirred for an hour at that temperature and then allowed to warm to room temperature. Subsequently, the reaction mixture was poured onto ice and the $p \mathrm{H}$ was carefully adjusted to 7.0 using concentrated aqueous $\mathrm{NH}_{3}$. The resulting slurry was stirred for an hour at $0{ }^{\circ} \mathrm{C}$ after which it was filtered and washed with ice-cold water. The crude product obtained was purified by silica gel flash column chromatography to obtain 5-bromoisoquinoline $\mathbf{1 n}{ }^{1}$ 
Scheme S2: Synthesis of arylisoquinolines:

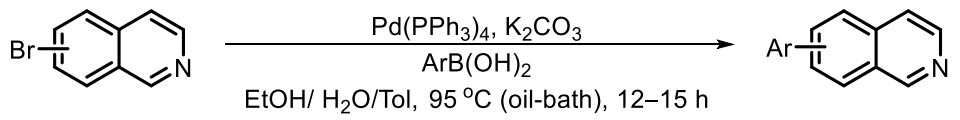

Bromoisoquinoline (250 mg, $1.2 \mathrm{mmol}, 1.0$ equiv) was dissolved in a mixture of $1.5 \mathrm{~mL} \mathrm{EtOH,} 3$ $\mathrm{mL}$ water and $6 \mathrm{~mL}$ toluene and the solution was degassed for $10 \mathrm{~min}$. To the resulting mixture, arylboronic acid (1.5 equiv), $\mathrm{K}_{2} \mathrm{CO}_{3}\left(662 \mathrm{mg}, 4.8 \mathrm{mmol}, 4.0\right.$ equiv) and $\mathrm{Pd}\left(\mathrm{PPh}_{3}\right)_{4}(65 \mathrm{mg}, 0.06$ mmol, 0.05 equiv) were added successively at room temperature. The reaction mixture was stirred at $95{ }^{\circ} \mathrm{C}$ (oil bath) under nitrogen for $12-15 \mathrm{~h}$. The progress of the transformation was monitored by TLC. Upon completion of the reaction, the reaction mixture was cooled to room temperature, passed through a pad of celite, followed by extraction with $\mathrm{CH}_{2} \mathrm{Cl}_{2}(2 \times 15 \mathrm{~mL})$. The organic layer was washed with brine and dried over anhydrous $\mathrm{Na}_{2} \mathrm{SO}_{4}$, filtered and concentrated under reduced pressure. The crude product was purified by silica gel flash column chromatography (eluted with 4:1, Petroleum ether: EtOAc).

5-(4-methoxyphenyl)isoquinoline (II): Prepared according to the general procedure and the title<smiles>COc1ccc(-c2cccc3cnccc23)cc1</smiles>
compound was isolated in $88 \%$ yield $(248 \mathrm{mg}$ ) as light-yellow oil. Spectral data obtained were in good agreement with those reported in the literature. ${ }^{2 a}{ }^{1} \mathbf{H}$ NMR (500 $\left.\mathrm{MHz}_{\mathrm{CDCl}}\right) \delta 9.31(\mathrm{~s}, 1 \mathrm{H}), 8.49(\mathrm{~s}, 1 \mathrm{H}), 8.02-7.91(\mathrm{~m}, 1 \mathrm{H}), 7.75(\mathrm{~d}, \mathrm{~J}=5.9 \mathrm{~Hz}$, 1H), $7.68-7.62(\mathrm{~m}, 2 \mathrm{H}), 7.44-7.38(\mathrm{~m}, 2 \mathrm{H}), 7.09-7.03(\mathrm{~m}, 2 \mathrm{H}), 3.90(\mathrm{~s}, 3 \mathrm{H}) ;{ }^{13} \mathrm{C}$

NMR (126 MHz, $\left.\mathrm{CDCl}_{3}\right) \delta 159.3,152.7,143.0,138.9,134.3,131.3,131.1,130.9,130.8,126.9$, $126.8,118.7,114.0,55.4$.

\section{5-(4-fluorophenyl)isoquinoline (Im):}

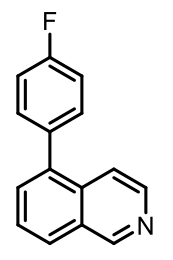

Prepared according to the general procedure and the title compound was isolated in $85 \%$ yield $(228 \mathrm{mg}$ ) as light-yellow oil. Spectral data obtained were in good agreement with those reported in the literature. ${ }^{2 b}{ }^{1} \mathbf{H} \mathbf{~ N M R}\left(400 \mathbf{M H z}, \mathrm{CDCl}_{3}\right) \delta 9.33$ (s, 1H), $8.52(\mathrm{~d}, \mathrm{~J}=6.0 \mathrm{~Hz}, 1 \mathrm{H}), 8.06-8.96(\mathrm{~m}, 1 \mathrm{H}), 7.73-7.61(\mathrm{~m}, 3 \mathrm{H}), 7.50-$ $7.42(\mathrm{~m}, 2 \mathrm{H}), 7.27-7.19(\mathrm{~m}, 2 \mathrm{H}) ;{ }^{13} \mathbf{C} \mathbf{N M R}\left(126 \mathrm{MHz}, \mathrm{CDCl}_{3}\right) \delta 163.5,161.5,152.9$, $143.5,138.1,134.9,134.9,134.1,131.4,131.4,130.9,128.9,127.3,126.8,118.2$, 115.6, $115.4,29.7$. 
5-phenylisoquinoline (Io): Prepared according to the general procedure and the title compound

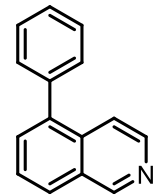
was isolated in $82 \%$ yield (208 mg) as light-yellow oil. Spectral data obtained were in good agreement with those reported in the literature. ${ }^{2 \mathrm{~d}}{ }^{\mathbf{1}} \mathbf{H} \mathbf{~ N M R}\left(500 \mathrm{MHz}, \mathrm{CDCl}_{3}\right) \delta$ $9.30(\mathrm{~s}, 1 \mathrm{H}), 8.48(\mathrm{~d}, \mathrm{~J}=6.0 \mathrm{~Hz}, 1 \mathrm{H}), 7.97-7.92(\mathrm{~m}, 1 \mathrm{H}), 7.74-7.69(\mathrm{~m}, 1 \mathrm{H}), 7.64$ - 7.60 (m, 2H), 7.52 - 7.47 (m, 2H), 7.46 - 7.41 (m, 3H); $\left.{ }^{13} \mathbf{C ~ N M R ~ ( 1 2 6 ~ M H z , ~ C D C l} 3\right) \delta 152.8$, $143.2,139.2,138.9,134.1,130.9,129.8,128.9,128.5,127.8,127.1,126.8,118.6$.

6-phenylisoquinoline (Ip): Prepared according to the general procedure and the title compound

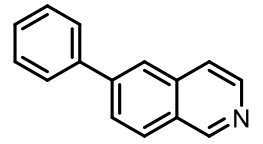
was isolated in $85 \%$ yield $(215 \mathrm{mg})$ as light-yellow oil. Spectral data obtained were in good agreement with those reported in the literature. ${ }^{2 e} \mathbf{1}^{1} \mathbf{H}$ NMR (500 $\mathrm{MHz}_{\mathrm{CDCl}} \mathrm{CD} 9.27(\mathrm{~s}, 1 \mathrm{H}), 8.55(\mathrm{~d}, \mathrm{~J}=5.8 \mathrm{~Hz}, 1 \mathrm{H}), 8.00(\mathrm{dd}, \mathrm{J}=8.5,2.5 \mathrm{~Hz}$, 1H), $7.97(\mathrm{~d}, \mathrm{~J}=1.9 \mathrm{~Hz}, 1 \mathrm{H}), 7.86-7.82(\mathrm{~m}, 1 \mathrm{H}), 7.72-7.68(\mathrm{~m}, 2 \mathrm{H}), 7.67(\mathrm{~d}, \mathrm{~J}=5.6 \mathrm{~Hz}, 1 \mathrm{H})$, $7.50(\mathrm{dd}, \mathrm{J}=8.3,6.9 \mathrm{~Hz}, 2 \mathrm{H}), 7.45-7.39(\mathrm{~m}, 1 \mathrm{H}) ;{ }^{13} \mathbf{C}$ NMR $\left(126 \mathrm{MHz}, \mathrm{CDCl}_{3}\right) \delta 152.2,143.3$, 143.0, 140.1, 136.1, 129.0, 128.2, 128.1, 127.7, 127.6, 127.1, 124.2, 120.7.

\section{6-(4-methoxyphenyl)isoquinoline (Iq):}

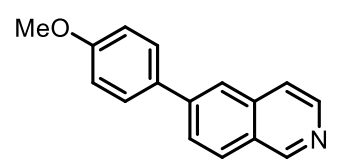

Prepared according to the general procedure and the title compound was isolated in $84 \%$ yield (237 mg) as light-yellow oil. Spectral data obtained were in good agreement with those reported in the literature. ${ }^{2 a}{ }^{1} \mathbf{H}$ NMR $\left(500 \mathrm{MHz}_{\mathrm{CDCl}}\right) \delta 9.24(\mathrm{~s}, 1 \mathrm{H}), 8.52(\mathrm{~d}, \mathrm{~J}=5.7 \mathrm{~Hz}, 1 \mathrm{H}), 7.98(\mathrm{~d}, \mathrm{~J}=8.5 \mathrm{~Hz}, 1 \mathrm{H}), 7.92(\mathrm{~d}, \mathrm{~J}=$ $1.8 \mathrm{~Hz}, 1 \mathrm{H}), 7.81(\mathrm{dd}, \mathrm{J}=8.6,1.8 \mathrm{~Hz}, 1 \mathrm{H}), 7.68-7.60(\mathrm{~m}, 3 \mathrm{H}), 7.06-6.99(\mathrm{~m}, 2 \mathrm{H}), 3.86(\mathrm{~s}$, 3H); ${ }^{13} \mathbf{C}$ NMR (126 MHz, $\left.\mathrm{CDCl}_{3}\right) \delta 159.8,152.2,143.4,142.6,136.2,132.5,128.6,128.0$, $127.4,126.8,123.2,120.5,114.4,55.4$.

\section{7-phenylisoquinoline (Is):}

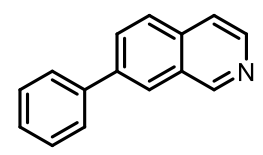

Prepared according to the general procedure and the title compound was isolated in $80 \%$ yield $(197 \mathrm{mg})$ as colorless oil. Spectral data obtained were in good agreement with those reported in the literature. ${ }^{2} \mathbf{H}$ NMR $\left(500 \mathrm{MHz}, \mathrm{CDCl}_{3}\right) \delta$ $9.31(\mathrm{~s}, 1 \mathrm{H}), 8.53(\mathrm{~d}, \mathrm{~J}=5.7 \mathrm{~Hz}, 1 \mathrm{H}), 8.18-8.13(\mathrm{~m}, 1 \mathrm{H}), 7.96(\mathrm{dd}, \mathrm{J}=8.5,1.8 \mathrm{~Hz}, 1 \mathrm{H}), 7.89$ $(\mathrm{d}, \mathrm{J}=8.5 \mathrm{~Hz}, 1 \mathrm{H}), 7.74-7.69(\mathrm{~m}, 2 \mathrm{H}), 7.67(\mathrm{dd}, \mathrm{J}=5.8,1.0 \mathrm{~Hz}, 1 \mathrm{H}), 7.51(\mathrm{dd}, \mathrm{J}=8.3,7.1 \mathrm{~Hz}$, 2H), 7.45 - $7.39(\mathrm{~m}, 1 \mathrm{H}) ;{ }^{13} \mathbf{C}$ NMR (126 MHz, $\left.\mathrm{CDCl}_{3}\right) \delta$ 152.8, 143.0, 140.2, 140.1, 134.9, $130.1,129.0,127.9,127.4,127.0,125.2,120.2$. 


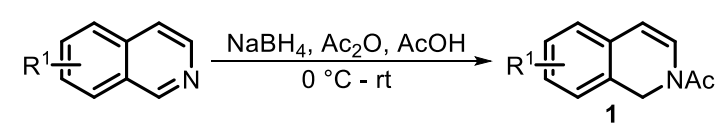

In a round-bottom flask, sodium borohydride (590 mg, $15.52 \mathrm{mmol}, 4.0$ equiv) was added portion-wise to a mixture of isoquinoline $(3.88 \mathrm{mmol})$, acetic anhydride $(2 \mathrm{~mL}, 20.56 \mathrm{mmol})$ and acetic acid $(6 \mathrm{~mL})$, over a period of $1.5 \mathrm{~h}$ at $0{ }^{\circ} \mathrm{C}$. After the addition was complete, the reaction mixture was warmed to room temperature over $1 \mathrm{~h}$. Upon completion of the reaction, it was diluted with water $(40 \mathrm{~mL})$, neutralized with sodium carbonate, and extracted with EtOAc $(2 \times 20$ $\mathrm{mL}$ ). The organic extract was dried over anhydrous $\mathrm{Na}_{2} \mathrm{SO}_{4}$ and concentrated under reduced pressure. The resulting crude mixture was purified by silica gel flash column chromatography (eluted with 4:1, Petroleum ether: EtOAc).

\section{1-(isoquinolin-2(1H)-yl)ethan-1-one (1b):}<smiles>C1=Cc2ccccc2CN1</smiles>

Prepared according to the general procedure and the title compound was isolated in $80 \%$ yield $(536 \mathrm{mg})$ as pale-yellow oil. Spectral data obtained were in good agreement with those reported in the literature. ${ }^{1}$

1-(5-(4-methoxyphenyl)isoquinolin-2(1H)-yl)ethan-1-one (mixture of rotamers) (11): Yield: 68\% ( 405 mg), Physical appearance: dark-yellow gel, TLC $R_{f} 0.40$ (4:1, Petroleum ether:

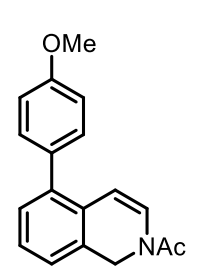
EtOAc); ${ }^{1} \mathbf{H}$ NMR (400 MHz, $\left.\mathrm{CDCl}_{3}\right) \delta 7.29$ - 7.27 (m, 2H), 7.23 - $7.20(\mathrm{~m}, 1 \mathrm{H})$, $7.16-7.14(\mathrm{~m}, 1 \mathrm{H}), 7.10-7.08(\mathrm{~m}, 1 \mathrm{H}), 6.99-6.95(\mathrm{~m}, 2 \mathrm{H}), 6.61(\mathrm{~d}, J=8.2 \mathrm{~Hz}$, $1 \mathrm{H}), 5.93(\mathrm{~d}, J=8.2 \mathrm{~Hz}, 1 \mathrm{H}), 4.98(\mathrm{~s}, 2 \mathrm{H}), 3.86(\mathrm{~s}, 3 \mathrm{H}), 2.20(\mathrm{~s}, 3 \mathrm{H}) ;{ }^{13} \mathbf{C}$ NMR $\left(126 \mathrm{MHz}, \mathrm{CDCl}_{3}\right) \delta 168.4,158.8,137.6,132.3,130.9,130.6,129.2,128.0,127.1$, 126.1, 124.8, 113.7, 108.3, 55.3, 44.6, 21.1; IR (KBr, $\left.\mathrm{cm}^{-1}\right): 2839,1768,1452,1341,1175$, 1064, 851, 701, 645; HRMS (ESI) $m / z:[\mathrm{M}+\mathrm{H}]^{+}$calcd. for $\mathrm{C}_{18} \mathrm{H}_{17} \mathrm{NO}_{2}$ 280.1332; found 280.1351 .

\section{1-(5-(4-fluorophenyl)isoquinolin-2(1H)-yl)ethan-1-one (1m):}

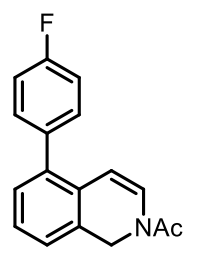

Prepared according to the general procedure and the title compound was isolated as a pale-yellow gel $59 \%$ yield $(590 \mathrm{mg})$. Spectral data obtained were in good agreement with those reported in the literature. ${ }^{2 c}$ 

Prepared according to the general procedure and the title compound was isolated in
$75 \%$ yield $(454 \mathrm{mg})$ as a pale-yellow gel. Spectral data obtained were in good
agreement with those reported in the literature. ${ }^{1}$

1-(5-phenylisoquinolin-2(1H)-yl)ethan-1-one (10): Prepared according to the general procedure and the title compound was isolated as a light yellow solid $60 \%$ yield $(579 \mathrm{mg})$. Spectral data obtained were in good agreement with those reported in the NAC iterature. ${ }^{2 \mathrm{~d}}$

1-(6-phenylisoquinolin-2(1H)-yl)ethan-1-one (mixture of rotamers) (1p):

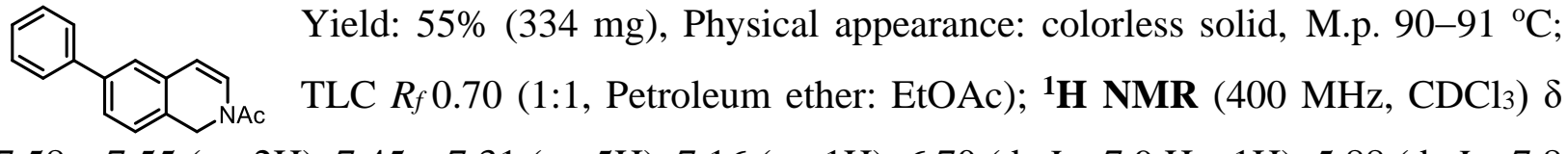
$7.58-7.55(\mathrm{~m}, 2 \mathrm{H}), 7.45-7.31(\mathrm{~m}, 5 \mathrm{H}), 7.16(\mathrm{~m}, 1 \mathrm{H}), 6.70(\mathrm{~d}, J=7.9 \mathrm{~Hz}, 1 \mathrm{H}), 5.88(\mathrm{~d}, J=7.8$ $\mathrm{Hz}, 1 \mathrm{H}), 4.98$ (s, 2H), 2.23 (s, 3H); ${ }^{13} \mathrm{C} \mathbf{N M R}\left(126 \mathrm{MHz}, \mathrm{CDCl}_{3}\right) \delta 168.5,140.8,130.8,129.0$, $128.8,128.5,128.2,127.6,127.4,126.9,126.4,126.4,126.1,123.4,109.6,44.2,21.2 ; \mathbf{I R}\left(\mathrm{cm}^{-1}\right)$ : 1773, 1665, 1625, 1427, 1378, 1339, 1300, 1136; HRMS (ESI) $m / z:[\mathrm{M}+\mathrm{Na}]^{+}$calcd. for $\mathrm{C}_{17} \mathrm{H}_{15} \mathrm{NONa} 272.1046$; found 272.1044.

1-(6-(4-methoxyphenyl)isoquinolin-2(1H)-yl)ethan-1-one (mixture of rotamers) (1q):

Yield: $78 \%$ (460 mg), Physical appearance: pale-yellow gel; TLC $R_{f} 0.60$ (1:1, Petroleum ether: EtOAc); ${ }^{1} \mathbf{H}$ NMR (500 MHz, $\left.\mathrm{CDCl}_{3}\right) \delta 7.53-$ $7.47(\mathrm{~m}, 2 \mathrm{H}), 7.35(\mathrm{~d}, J=7.8,1.9 \mathrm{~Hz}, 1 \mathrm{H}), 7.22(\mathrm{~d}, J=1.9 \mathrm{~Hz}, 1 \mathrm{H}), 7.14$ $(\mathrm{d}, J=7.8 \mathrm{~Hz}, 1 \mathrm{H}), 7.01-6.93(\mathrm{~m}, 2 \mathrm{H}), 6.70(\mathrm{~d}, J=7.8 \mathrm{~Hz}, 1 \mathrm{H}), 5.88(\mathrm{~d}, J=7.9 \mathrm{~Hz}, 1 \mathrm{H}), 4.97$ (s, 2H), 3.85 (s, 3H), 2.23 (s, 3H); $\left.{ }^{13} \mathrm{C} \mathrm{NMR} \mathrm{(126} \mathrm{MHz,} \mathrm{CDCl}_{3}\right) \delta$ 168.5, 159.2, 140.4, 133.1, 130.8, 127.9, 126.4, 126.3, 125.6, 122.9, 114.2, 109.6, 55.3, 44.2, 21.3; IR $\left(\mathrm{cm}^{-1}\right)$ : 2956, 2916, 2838, 2090, 1660, 1604, 1524, 1435, 1352, 1239; HRMS (ESI) $m / z:[\mathrm{M}+\mathrm{Na}]^{+}$calcd. for $\mathrm{C}_{18} \mathrm{H}_{17} \mathrm{NO}_{2} \mathrm{Na} 302.1151$; found 302.1136.

1-(6-bromoisoquinolin-2(1H)-yl)ethan-1-one (mixture of rotamers) (1r): Reaction set on 1 (1) mmol scale; Yield: 65\% (198 mg), Physical appearance: light-brown solid, M.p. 50-52 ${ }^{\circ} \mathrm{C}$; TLC $R_{f} 0.50$ (7:3, Petroleum ether: EtOAc); ${ }^{1} \mathbf{H}$ NMR (400 MHz, 
$\left.\mathrm{CDCl}_{3}\right) \delta 7.32-7.24(\mathrm{~m}, 3 \mathrm{H}), 7.17(\mathrm{~d}, \mathrm{~J}=2.1 \mathrm{~Hz}, 1 \mathrm{H}), 6.96(\mathrm{~d}, \mathrm{~J}=8.1 \mathrm{~Hz}, 1 \mathrm{H}), 6.70(\mathrm{~d}, \mathrm{~J}=7.9$ $\mathrm{Hz}, 1 \mathrm{H}), 5.77-5.71(\mathrm{~d}, 7.82 \mathrm{~Hz}, 1 \mathrm{H}), 4.88(\mathrm{~s}, 2 \mathrm{H}), 2.22(\mathrm{~s}, 3 \mathrm{H}) ;{ }^{13} \mathrm{C}$ NMR $\left(126 \mathrm{MHz}, \mathrm{CDCl}_{3}\right) \delta$ $168.4,132.5,129.9,128.1,127.4,127.3,121.3,119.4,108.2,43.9,21.2 ; \mathbf{I R}\left(\mathrm{cm}^{-1}\right): 2854,1745$, 1664, 1620, 1590, 1561, 1431, 1375, 1209; HRMS (ESI) $m / z:[\mathrm{M}+\mathrm{H}]^{+}$calcd. for $\mathrm{C}_{11} \mathrm{H}_{11} \mathrm{BrNO}$ 252.0019 and 253.9998; found 252.0016 and 253.9998.

\section{1-(7-phenylisoquinolin-2(1H)-yl)ethan-1-one (mixture of rotamers) $(1 \mathrm{~s})$ :}

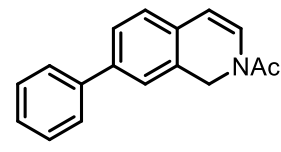

Yield: $71 \%$ (431 mg), Physical appearance: colorless gel; TLC $R_{f} 0.7$ (1:1,

Petroleum ether: EtOAc); ${ }^{1} \mathbf{H}$ NMR $\left(500 \mathrm{MHz}, \mathrm{CDCl}_{3}\right) \delta 7.59-7.55$ (m, 2H), $7.44(\mathrm{dd}, \mathrm{J}=8.5,6.8 \mathrm{~Hz}, 3 \mathrm{H}), 7.38-7.35(\mathrm{~m}, 1 \mathrm{H}), 7.35-7.32(\mathrm{~m}, 1 \mathrm{H}), 7.10$ $(\mathrm{d}, \mathrm{J}=7.8 \mathrm{~Hz}, 1 \mathrm{H}), 6.66(\mathrm{~d}, \mathrm{~J}=7.8 \mathrm{~Hz}, 1 \mathrm{H}), 5.86(\mathrm{~d}, \mathrm{~J}=7.8 \mathrm{~Hz}, 1 \mathrm{H}), 5.01(\mathrm{~s}, 2 \mathrm{H}), 2.22(\mathrm{~s}, 3 \mathrm{H})$; ${ }^{13} \mathrm{C}$ NMR $\left(126 \mathrm{MHz}, \mathrm{CDCl}_{3}\right) \delta 168.5,140.4,140.2,135.6,130.0,129.6,128.8,128.0,127.4$, 126.8, 126.3, 126.2, 125.1, 124.7, 109.3, 44.5, 21.3; IR $\left(\mathrm{cm}^{-1}\right): 2926,1660,1625,1487,1419$, 1383, 1260, 1074; HRMS (ESI) $m / z:[\mathrm{M}+\mathrm{H}]^{+}$calcd. for $\mathrm{C}_{17} \mathrm{H}_{16} \mathrm{NO} 250.1226$; found 250.1229.

\section{1-(7-bromoisoquinolin-2(1H)-yl)ethan-1-one (mixture of rotamers) (1t) :}

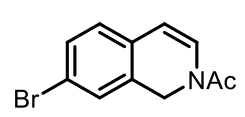

Yield: 88\% (532 mg), Physical appearance: dark-yellow solid, M.p. 110-112

${ }^{\circ} \mathrm{C}$, TLC $R_{f} 0.40$ (4:1, Petroleum ether: EtOAc); ${ }^{1} \mathbf{H}$ NMR $\left(500 \mathrm{MHz}, \mathrm{CDCl}_{3}\right) \delta$ $7.35-7.28(\mathrm{~m}, 1 \mathrm{H}), 7.25-7.20(\mathrm{~m}, 1 \mathrm{H}), 6.89(\mathrm{~d}, \mathrm{~J}=8.0 \mathrm{~Hz}, 1 \mathrm{H}), 6.67$ (d, J = 7.8 Hz, 1H), 5.77 $(\mathrm{d}, \mathrm{J}=7.8 \mathrm{~Hz}, 1 \mathrm{H}), 4.90(\mathrm{~s}, 2 \mathrm{H}), 2.21(\mathrm{~s}, 3 \mathrm{H}) ;{ }^{13} \mathbf{C ~ N M R}\left(126 \mathrm{MHz}, \mathrm{CDCl}_{3}\right) \delta$ 168.4, 131.3, 131.1, 130.7, 129.5, 128.9, 128.5, 126.6, 126.0, 120.6, 108.6, 108.6, 43.8, 21.2; IR $\left(\mathrm{KBr}, \mathrm{cm}^{-1}\right)$ : 2729, 1668, 1452, 1255, 1144, 1064; HRMS (ESI) $m / z:[\mathrm{M}+\mathrm{H}]^{+}$calcd. for $\mathrm{C}_{18} \mathrm{H}_{17} \mathrm{NO} 252.0019$ and 253.9998; found 252.0021 and 253.9999.

\section{1-(3-methylisoquinolin-2(1H)-yl)ethan-1-one (1u):}

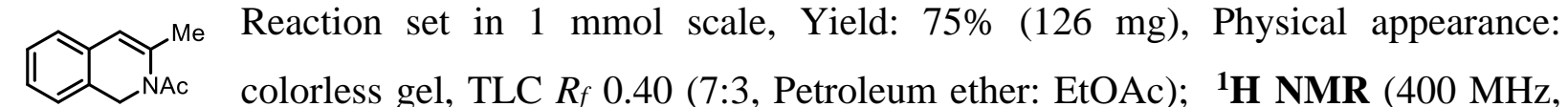
$\left.\mathrm{CDCl}_{3}\right) \delta 9.17(\mathrm{~s}, 1 \mathrm{H}), 7.92(\mathrm{~d}, J=8.2 \mathrm{~Hz}, 1 \mathrm{H}), 7.72(\mathrm{~d}, J=8.3 \mathrm{~Hz}, 1 \mathrm{H}), 7.67-7.60(\mathrm{~m}, 1 \mathrm{H})$, $7.52(\mathrm{t}, J=7.5 \mathrm{~Hz}, 1 \mathrm{H}), 7.48(\mathrm{~s}, 1 \mathrm{H}), 2.70(\mathrm{~s}, 3 \mathrm{H}) ;{ }^{13} \mathbf{C} \mathbf{N M R}\left(126 \mathrm{MHz}, \mathrm{CDCl}_{3}\right) \delta 151.9,151.6$, 136.6, 130.3, 127.5, 126.8, 126.2, 125.9, 118.4, 24.1; IR (cm $\left.{ }^{-1}\right): 2801,1685,1499,1358,1205$; HRMS (ESI) $m / z:[\mathrm{M}-\mathrm{Ac}]^{+}$calcd. for $\mathrm{C}_{10} \mathrm{H}_{10} \mathrm{~N}$ 144.0808; found 144.0802. 
Scheme S4: General procedure for synthesis of 1-alkyl/aryl- $N$-acetyl-1,2dihydroisoquinolines: ${ }^{1}$

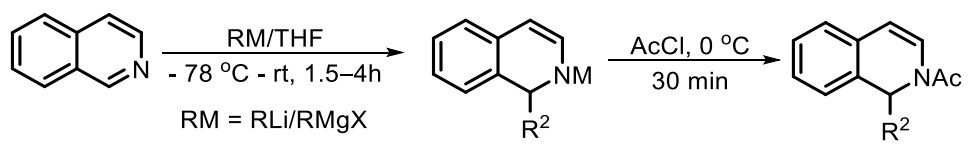

To the solution of isoquinoline $(2.0 \mathrm{mmol})$ in THF $(5 \mathrm{~mL})$ under an argon atmosphere, a solution of the Grignard reagent or organolithium reagent (1.5 equiv.) was added dropwise at $-78^{\circ} \mathrm{C}$. The temperature was gradually allowed to increase to $25{ }^{\circ} \mathrm{C}$ over 30 minutes and the reaction mixture was stirred for another hour, following which the resulting mixture was cooled to $0{ }^{\circ} \mathrm{C}$. Thereafter, acetyl chloride $(0.3 \mathrm{~mL}, 3.5 \mathrm{mmol})$ was added dropwise to the reaction mixture. The solution was stirred for another 30 minutes at room temperature and then diluted with saturated solution of $\mathrm{NH}_{4} \mathrm{Cl}$. This mixture was extracted with ethyl acetate $(3 \times 10 \mathrm{~mL})$ and washed successively with brine. The organic layer was dried over anhydrous $\mathrm{Na}_{2} \mathrm{SO}_{4}$, filtered and concentrated under reduced pressure. The crude product was purified by silica gel flash column chromatography (eluated with Petroleum ether: EtOAc).

\section{1-(1-phenylisoquinolin-2(1H)-yl)ethan-1-one (1a):}

Prepared according to the general procedure and the title compound was isolated in $85 \%$ yield (423 mg) as a pale-yellow solid. Spectral data obtained were in good agreement with those reported in the literature. ${ }^{1}$

\section{1-(1-methylisoquinolin-2(1H)-yl)ethan-1-one (1c):}<smiles>CN1C=Cc2ccccc2C1[N+](=O)[O-]</smiles>

Prepared according to the general procedure and the title compound was isolated in $85 \%$ yield $(318 \mathrm{mg})$ as colourless oil. Spectral data obtained were in good agreement with those reported in the literature. ${ }^{1}$

\section{(1-(1-isopropyl)isoquinolin-2(1H)-yl)ethan-1-one (1d):}

Prepared according to the general procedure and the title compound was isolated in
$78 \%$ yield $(335 \mathrm{mg})$ as a colorless solid. Spectral data obtained were in good agreement with those reported in the literature. ${ }^{1}$ 
1-(1-ethylisoquinolin-2(1H)-yl)ethan-1-one (1e):

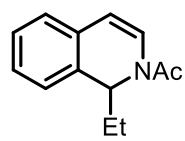

Prepared according to the general procedure and the title compound was isolated as $79 \%$ yield $(318 \mathrm{mg})$ as a pale-yellow solid. Spectral data obtained were in good agreement with those reported in the literature. ${ }^{1}$

\section{(1-(1-butyl)isoquinolin-2(1H)-yl)ethan-1-one (1f):}

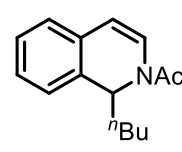

Prepared according to the general procedure and the title compound was isolated in $78 \%$ yield $(357 \mathrm{mg})$ as a pale-yellow oil. Spectral data obtained were in good agreement with those reported in the literature. ${ }^{1}$

\section{1-(1-(tert-butyl)isoquinolin-2(1H)-yl)ethan-1-one (1g):}

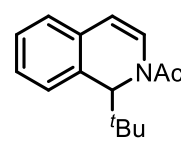

Yield: 62\% (284 mg), Physical appearance: pale-yellow oil, TLC $R_{f} 0.40$ (4:1,

Petroleum ether: EtOAc); ${ }^{1} \mathbf{H}$ NMR (400 MHz, $\left.\mathrm{CDCl}_{3}\right) \delta 7.27-7.20(\mathrm{~m}, 2 \mathrm{H}), 7.15$ $-7.09(\mathrm{~m}, 2 \mathrm{H}), 6.72(\mathrm{dd}, J=7.6,1.3 \mathrm{~Hz}, 1 \mathrm{H}), 5.98(\mathrm{~d}, J=7.6 \mathrm{~Hz}, 1 \mathrm{H}), 5.60(\mathrm{~d}, J=1.2 \mathrm{~Hz}, 1 \mathrm{H})$, 2.24 (s, 3H), 0.89 (s, 9H); ${ }^{13} \mathbf{C}$ NMR (126 MHz, $\left.\mathrm{CDCl}_{3}\right) \delta$ 169.5, 131.3, 130.3, 128.8, 127.4, 126.7, 126.5, 124.3, 112.7, 60.1, 39.0, 26.8, 21.8; IR (KBr, cm $\left.{ }^{-1}\right): 2894,1829,1664,1452,1164$, 1022, 854, 745; HRMS (ESI) $m / z:[\mathrm{M}+\mathrm{H}]^{+}$calcd. for $\mathrm{C}_{15} \mathrm{H}_{19} \mathrm{NO} 230.1539$; found 230.1560 .

\section{Scheme S5: General procedure for synthesis of $\alpha$-diazomalonates: ${ }^{3}$}

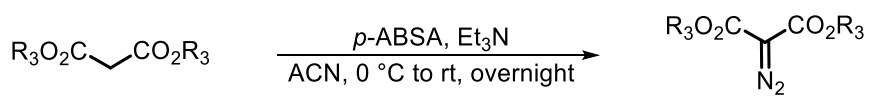

To a solution of the corresponding ester $(5 \mathrm{mmol})$ in acetonitrile $(6 \mathrm{~mL}), \mathrm{Et}_{3} \mathrm{~N}$ (3 equiv.) was added at $0{ }^{\circ} \mathrm{C}$. The resulting solution was stirred for $5 \mathrm{mins}$, followed by the addition of 4acetamidobenzenesulfonyl azide ( $p-\mathrm{ABSA})(5 \mathrm{mmol})$. Subsequently, the cooling bath was removed, and the mixture was stirred overnight at room temperature. The solvent was evaporated, and the resulting residue was diluted with water, extracted with ethyl acetate $(2 \times 10$ $\mathrm{mL}$ ) and the organic extract was dried over anhydrous $\mathrm{Na}_{2} \mathrm{SO}_{4}$. The solvent was removed under reduced pressure, and the resulting residue was purified by silica gel flash column chromatography. ${ }^{3}$

\section{Dimethyl 2-diazomalonate (2a):}

$\begin{array}{ll}\mathrm{MeO}_{2} \mathrm{C} & \mathrm{N}_{\mathrm{CO}_{2} \mathrm{Me}} \\ & \text { Prepared according to the general procedure and the title compound was isolated } \\ \text { in } 90 \% \text { yield }(711 \mathrm{mg}) \text { as a light-yellow oil. Spectral data obtained were in good }\end{array}$ 
agreement with those reported in the literature. ${ }^{3,5 \mathrm{c}}$

Diethyl 2-diazomalonate (2h):

${ }_{\mathrm{EtO}_{2} \mathrm{C}} \stackrel{\mathrm{N}_{2}}{\mathrm{CO}_{2} \mathrm{Et}} \begin{aligned} & \text { Prepared according to the general procedure on } 1 \mathrm{mmol} \text { scale and the title } \\ & \text { compound was isolated in } 86 \% \text { yield }(160 \mathrm{mg}) \text { as a light-yellow oil. Spectral data }\end{aligned}$ obtained were in good agreement with those reported in the literature. ${ }^{3,5 \mathrm{c}}$

\section{Diisopropyl 2-diazomalonate (2i):}

${ }_{\mathrm{PrO}_{2} \mathrm{C}} \stackrel{\mathrm{N}_{2}}{\stackrel{\text { Prepared according to the }}{\mathrm{N}} \text { compound was isolated in } 88 \% \text { yield }(200 \mathrm{mg}) \text { as a light-yellow oil. Spectral data }}$ obtained were in good agreement with those reported in the literature. ${ }^{3,5 \mathrm{c}}$

\section{Dibenzyl 2-diazomalonate (2j):}

$\mathrm{BnO}_{2} \mathrm{C}{ }_{\mathrm{CO}_{2} \mathrm{Bn}}^{\mathrm{N}_{2}} \begin{aligned} & \text { Prepared according to the general procedure on } 1 \mathrm{mmol} \text { scale and the title } \\ & \text { compound was isolated as a colorless solid } 88 \% \text { yield }(272 \mathrm{mg}) \text {. Spectral data }\end{aligned}$ obtained were in good agreement with those reported in the literature. ${ }^{3,5 \mathrm{c}}$

\section{Di-tert-butyl 2-diazomalonate (2k):}

$\mathrm{N}_{2} \quad$ Prepared according to the general procedure on $1 \mathrm{mmol}$ scale and the title ${ }_{\mathrm{BuO}_{2} \mathrm{C}} \mathrm{H}_{\mathrm{CO}_{2}{ }_{2} \mathrm{Bu}}$ compound was isolated in $85 \%$ yield $(200 \mathrm{mg})$ as a colorless solid. Spectral data obtained were in good agreement with those reported in the literature. ${ }^{5 \mathrm{c}}$

Scheme S6. General procedure for the synthesis of $\alpha$-diazotized Meldrum's acid (5-diazo-2, 2-dimethyl-1,3-dioxane-4,6-dione (4)): ${ }^{4}$
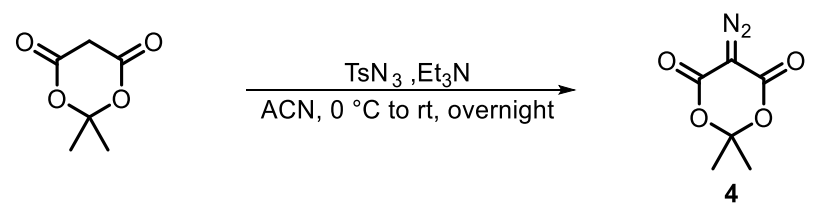

To a solution of Meldrum's acid (1g, $4.4 \mathrm{mmol})$ in acetonitrile $(12 \mathrm{~mL}), \mathrm{Et}_{3} \mathrm{~N}(0.92 \mathrm{ml}, 6.6$ mmol) was added at $0{ }^{\circ} \mathrm{C}$. The resulting solution was stirred for 5 minutes, followed by the addition of tosyl azide (1.73 g, $8.8 \mathrm{mmol})$. Subsequently, the cooling bath was removed, and the mixture was stirred overnight at room temperature. The solvent was evaporated, and the resulting residue was diluted with water, extracted with ethyl acetate $(2 \times 15 \mathrm{~mL})$ and the organic extract 
was dried over anhydrous $\mathrm{Na}_{2} \mathrm{SO}_{4}$. The organic solvent was removed under reduced pressure, and the resulting residue was purified by silica gel flash column chromatography (eluted with 8:2, Petroleum ether: EtOAc). Yield: $78 \%$ (924 mg), Physical appearance: pale-yellow solid, $\mathrm{R}_{f}$ $=0.3$. Spectral data obtained were in good agreement with those reported in the literature. ${ }^{4}$

\section{Scheme S7: General Procedure for the synthesis of $\beta$-keto ester: ${ }^{5}$}

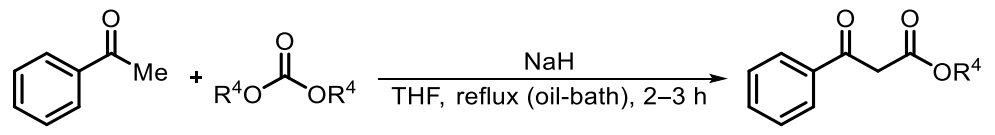

In a round bottom flask, $\mathrm{NaH}(384 \mathrm{mg}, 16.0 \mathrm{mmol}, 4.0$ equiv) was added along with dialkyl carbonate (16.0 mmol, 4.0 equiv), and THF $(100 \mathrm{~mL})$ and the reaction mixture was stirred at room temperature. A solution of corresponding ketone (4.0 mmol, 1.0 equiv) in THF (20 mL) was added dropwise over 30 mins. After the addition, the mixture was heated to reflux (oil bath) until the evolution of hydrogen ceased (15-20 min). Upon completion of the reaction and subsequent cooling to room temperature, THF was evaporated. The mixture was diluted with water $(20 \mathrm{~mL})$ and extracted with EtOAc $(15 \mathrm{~mL} \times 2)$. The extract was washed with brine, dried over anhydrous $\mathrm{Na}_{2} \mathrm{SO}_{4}$ and concentration under reduced pressure. The residue was purified by silica gel flash column chromatography (eluted with 9:1, Petroleum ether: EtOAc) and the desired $\beta$-keto ester was obtained with $80-95 \%$ yield.

\section{Scheme S8: General procedure for synthesis of $\alpha$-diazo- $\beta$-ketoesters: ${ }^{5}$}

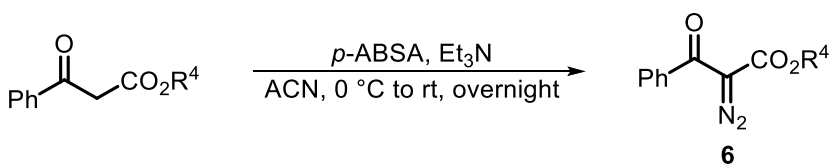

To a solution of $\beta$-keto ester $(1.3 \mathrm{mmol})$ in anhydrous $\mathrm{CH}_{3} \mathrm{CN}$ (5 mL), 4-toluenesulfonyl azide (343 $\mathrm{mg}, 1.43 \mathrm{mmol}$ ) was added and the reaction mixture was cooled to $0{ }^{\circ} \mathrm{C}$. The resulting mixture was stirred at $0{ }^{\circ} \mathrm{C}$ and then solution of $\mathrm{Et} 3 \mathrm{~N}(0.5 \mathrm{~mL}, 3.9 \mathrm{mmol})$ in $15 \mathrm{~mL} \mathrm{CH} 3 \mathrm{CN}$ was added dropwise. Next, the cooling bath was removed, and the mixture was stirred at room temperature for $4 \mathrm{~h}$. The solvent was evaporated, and the resulting residue was diluted with water and extracted with EtOAc $(2 \times 10 \mathrm{~mL})$. The combined extract was washed with brine, dried over anhydrous $\mathrm{Na}_{2} \mathrm{SO}_{4}$ and concentrated under reduced pressure. The residue was purified by silica gel flash column chromatography (eluted with 9:1, Petroleum ether: EtOAc) and the desired $\alpha$ diazo- $\beta$-keto compounds were obtained in good yields. 


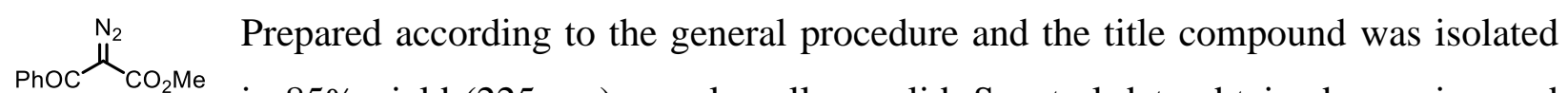
in $85 \%$ yield $(225 \mathrm{mg})$ as pale-yellow solid. Spectral data obtained were in good agreement with those reported in the literature. ${ }^{5}$

Ethyl-2-diazo-3-oxo-3-phenylpropanoate (6b): $\begin{array}{cl}\text { PhOC }^{\mathrm{N}_{2}} & \text { Prepared according to the general procedure and the title compound was isolated in } \\ \mathrm{CO}_{2} \mathrm{Et} & 87 \% \text { yield }(247 \mathrm{mg}) \text { as pale-yellow solid. Spectral data obtained were in good }\end{array}$ agreement with those reported in the literature. ${ }^{5}$

\section{Scheme S9: Synthesis of $\beta$-keto ester (benzyl 3-oxo-3-phenylpropanoate): ${ }^{6 a, b}$ \\ General Procedure:}

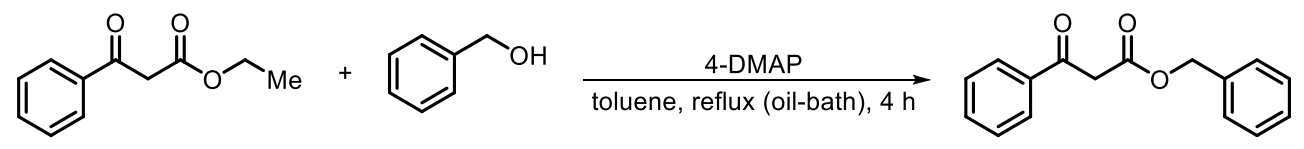

To a solution of ethyl 3-oxo-3-phenylpropanoate (500 mg, $2.27 \mathrm{mmol}, 1.0$ equiv) and phenylmethanol (295 mg, $2.72 \mathrm{mmol}, 1.2$ equiv) in toluene (10 mL) and the reaction mixture was stirred at room temperature. This was followed by the addition of DMAP (56 mg, 0.45 mmol, 0.2 equiv) and the resulting mixture was refluxed at $110^{\circ} \mathrm{C}$ in a preheated oil bath until the consumption of ethyl 3-oxo-3-phenylpropanoate (monitored by TLC). The mixture was diluted with water $(20 \mathrm{~mL})$ and extracted with EtOAc $(2 \times 15 \mathrm{~mL})$. The combined extract was washed with brine, dried over anhydrous $\mathrm{Na}_{2} \mathrm{SO}_{4}$ and concentrated under reduced pressure. The residue was purified by silica gel flash column chromatography (eluted with 9:1, Petroleum ether: EtOAc). The benzyl 3-oxo-3-phenylpropanoate was obtained as pale-yellow oil. Yield: $80 \%$ (405 mg).

Scheme S10: Synthesis of benzyl 2-diazo-3-oxo-3-phenylpropanoate ( $\alpha$-diazo- $\beta$-keto) (6c):

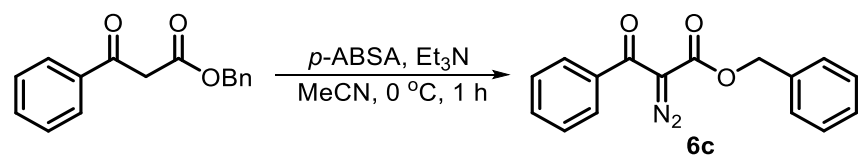

Procedure described in Scheme S8 was followed. Yield 78\% (348 mg), Physical appearance: dark yellow oil, TLC $R_{f} 0.50$ (9:1, Petroleum ether: EtOAc); ${ }^{1} \mathbf{H}$ NMR (400 MHz, $\left.\mathrm{CDCl}_{3}\right) \delta 7.65$ - $7.61(\mathrm{~m}, 2 \mathrm{H}), 7.55-7.50(\mathrm{~m}, 1 \mathrm{H}), 7.45-7.39(\mathrm{~m}, 2 \mathrm{H}), 7.3-7.33(\mathrm{~m}, 3 \mathrm{H}), 7.29-7.27$ (m, Page $\mathrm{S} 12$ of $\mathrm{S} 103$ 
2H), $5.22(\mathrm{~s}, 2 \mathrm{H}) ;{ }^{13} \mathbf{C}$ NMR $\left(126 \mathrm{MHz}, \mathrm{CDCl}_{3}\right) \delta 160.8,135.2,128.6,128.4,128.2$, 67.1; IR $\left(\mathrm{KBr}, \mathrm{cm}^{-1}\right): 2972,1870,1711,1657,1401,1189,1060$; HRMS (ESI) $m / z:[\mathrm{M}+\mathrm{H}]^{+}$calcd. for $\mathrm{C}_{16} \mathrm{H}_{12} \mathrm{~N}_{2} \mathrm{O}_{3} 281.0921$; found 281.0926.

Scheme S11: General procedure for the synthesis of $\alpha$-diazodiketones:
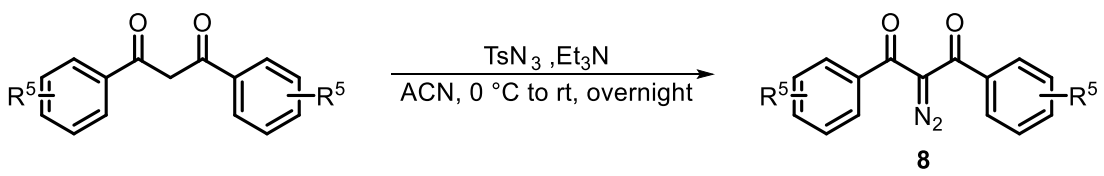

To a solution of the corresponding 1,3-diaryl-1,3-propanediones ${ }^{7}(1.0 \mathrm{mmol})$ in acetonitrile (3 $\mathrm{mL}), \mathrm{Et}_{3} \mathrm{~N}(0.2 \mathrm{ml}, 1.5 \mathrm{mmol})$ was added at $0{ }^{\circ} \mathrm{C}$. The resulting solution was stirred for 5 minutes, followed by the addition of tosyl azide $(280 \mathrm{mg}, 1.5 \mathrm{mmol})$. Subsequently, the cooling bath was removed, and the mixture was stirred overnight at room temperature. The solvent was evaporated, and the resulting residue was diluted with water, extracted with ethyl acetate $(2 \times 5$ $\mathrm{mL}$ ) and the organic extract was dried over anhydrous $\mathrm{Na}_{2} \mathrm{SO}_{4}$. The organic solvent was removed under reduced pressure, and the resulting residue was purified by silica gel flash column chromatography (eluted with Petroleum ether: EtOAc).

\section{2-diazo-1,3-diphenylpropane-1,3-dione (8a):}

Phoc $\stackrel{\mathrm{N}}{\mathrm{NOPh}}_{\text {COP }}$ in $80 \%$ yield $(200 \mathrm{mg})$ as a pale-yellow solid. Spectral data obtained were in good agreement with those reported in the literature. ${ }^{7} \mathbf{~} \mathbf{H}$ NMR $\left(400 \mathrm{MHz}, \mathrm{CDCl}_{3}\right) \delta 7.57(\mathrm{dd}, \mathrm{J}=8.2$, $1.3 \mathrm{~Hz}, 4 \mathrm{H}), 7.48-7.40(\mathrm{~m}, 2 \mathrm{H}), 7.32(\mathrm{t}, \mathrm{J}=7.7 \mathrm{~Hz}, 4 \mathrm{H}) ;{ }^{13} \mathbf{C} \mathbf{N M R}\left(126 \mathrm{MHz}, \mathrm{CDCl}_{3}\right) \delta 186.4$, $136.9,132.6,128.4,128.3$.

\section{2-diazo-1,3-bis(4-methoxyphenyl)propane-1,3-dione(8c):}

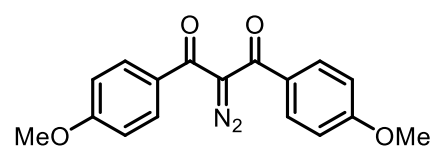

Yield: 62\% (172 mg), Physical appearance: yellow solid, M.p. 94-95 ${ }^{\circ} \mathrm{C}$; TLC $\mathrm{R}_{f} 0.3$ (1:1, Petroleum ether: EtOAc); ${ }^{1} \mathbf{H}$ NMR $(500 \mathrm{MHz}$, $\left.\mathrm{CDCl}_{3}\right) \delta 7.60(\mathrm{~d}, \mathrm{~J}=8.8 \mathrm{~Hz}, 4 \mathrm{H}), 6.83(\mathrm{~d}, \mathrm{~J}=8.8 \mathrm{~Hz}, 4 \mathrm{H}), 3.82$ (s, $6 \mathrm{H}) ;{ }^{13} \mathbf{C}$ NMR $\left(126 \mathrm{MHz}, \mathrm{CDCl}_{3}\right) \delta 185.2,163.2,130.8,129.5,113.6,55.4 ; \mathbf{I R}\left(\mathrm{cm}^{-1}\right): 2939$, 2845, 2162, 1639, 1598, 1295, 1174; HRMS (ESI) $m / z:[\mathrm{M}+\mathrm{Na}]^{+}$calcd. for $\mathrm{C}_{17} \mathrm{H}_{14} \mathrm{~N}_{2} \mathrm{O}_{4} \mathrm{Na}$ 333.0846; found 333.0862 . 
Scheme S12: Procedure for the synthesis of 2-diazo-5,5-dimethylcyclohexane-1,3-dione-5,5dimethylcyclohexane-1,3-dione (8f): ${ }^{8}$
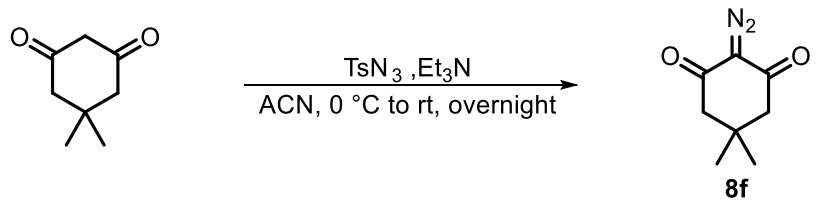

To a solution of dimedone $(0.2 \mathrm{~g}, 1.428 \mathrm{mmol})$ in acetonitrile $(3 \mathrm{~mL}), \mathrm{Et}_{3} \mathrm{~N}(0.3 \mathrm{ml}, 2.142 \mathrm{mmol})$ was added at $0{ }^{\circ} \mathrm{C}$. The resulting solution was stirred for 5 minutes, followed by the addition of tosyl azide (400 mg, $2.142 \mathrm{mmol}$ ). Subsequently, the cooling bath was removed, and the mixture was stirred overnight at room temperature. The solvent was evaporated, and the resulting residue was diluted with water, extracted with ethyl acetate $(2 \times 5 \mathrm{~mL})$ and the organic extract was dried over anhydrous $\mathrm{Na}_{2} \mathrm{SO}_{4}$. The organic solvent was removed under reduced pressure, and the resulting residue was purified by silica gel flash column chromatography (8:2, Petroleum ether: EtOAc). Compound was isolated as yellow solid 82\% yield (194 mg). Spectral data obtained were in good agreement with those reported in the literature. ${ }^{8}$

\section{iii) Optimization Studies:}

Table S1: Optimization of reaction conditions for $\alpha$-diazomalonates:

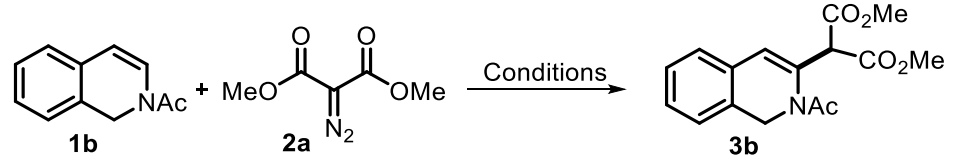

\begin{tabular}{|c|c|c|}
\hline Entry & Conditions & $\begin{array}{l}\text { Yield of 3b } \\
\qquad(\%)^{a}\end{array}$ \\
\hline 1 & {$\left[\mathrm{Cp}^{*} \mathrm{RhCl}_{2}\right]_{2}(1 \mathrm{~mol} \%) / \mathrm{AgBF}_{4}(4 \mathrm{~mol} \%) / \mathrm{DCE} / 40^{\circ} \mathrm{C} / 6 \mathrm{~h}$} & NR \\
\hline 2 & $\begin{array}{l}{\left[\mathrm{Cp}^{*} \mathrm{RhCl}_{2}\right]_{2}(3 \mathrm{~mol} \%) / \mathrm{AgSbF}_{6}(12 \mathrm{~mol} \%) / \mathrm{AcOH}(0.5 \text { equiv }) / \mathrm{DCE} / 40} \\
{ }^{\circ} \mathrm{C} / 6 \mathrm{~h}\end{array}$ & NR \\
\hline 3 & $\begin{array}{l}{\left[\mathrm{Cp}^{*} \mathrm{RhCl}_{2}\right]_{2}(2.5 \mathrm{~mol} \%) / \mathrm{AgSbF}_{6}(10 \mathrm{~mol} \%) / \mathrm{PivOH}(0.5} \\
\text { equiv }) / \mathrm{MeOH} / 40{ }^{\circ} \mathrm{C} / 10 \mathrm{~h}\end{array}$ & NR \\
\hline 4 & $\mathrm{Cp}^{*} \mathrm{Co}(\mathrm{CO}) \mathrm{I}_{2}(5 \mathrm{~mol} \%) / \mathrm{AgSbF}_{6}(10 \mathrm{~mol} \%) / \mathrm{DCE} / 100{ }^{\circ} \mathrm{C} / 15 \mathrm{~h}$ & NR \\
\hline 5 & {$\left[\mathrm{Cp}^{*} \mathrm{RhCl}_{2}\right]_{2}(5 \mathrm{~mol} \%) / \mathrm{AgOAc}(20 \mathrm{~mol} \%) / \mathrm{DCE} / 40-60{ }^{\circ} \mathrm{C} / 6 \mathrm{~h}$} & NR \\
\hline 6 & {$\left[\mathrm{Cp}^{*} \mathrm{RhCl}_{2}\right]_{2}(1 \mathrm{~mol} \%) / \mathrm{AgOAc}(7.5 \mathrm{~mol} \%) / \mathrm{MeOH} / 40-60^{\circ} \mathrm{C} / 6 \mathrm{~h}$} & NR \\
\hline
\end{tabular}




\begin{tabular}{|c|c|c|}
\hline 7 & $\begin{array}{l}{\left[\mathrm{Cp}^{*} \mathrm{IrCl}_{2}\right]_{2}(3 \mathrm{~mol} \%) / \mathrm{AgBF}_{4}(12 \mathrm{~mol} \%) / \mathrm{TMBzOH}(0.2} \\
\text { equiv }) / \mathrm{HFIP} / 60^{\circ} \mathrm{C} / 6 \mathrm{~h}\end{array}$ & 66 \\
\hline 8 & $\begin{array}{l}{\left[\mathrm{Cp}^{*} \mathrm{IrCl}_{2}\right]_{2}(3 \mathrm{~mol} \%) / \mathrm{AgSbF}_{6}(12 \mathrm{~mol} \%) / \mathrm{TMBzOH}(0.2} \\
\text { equiv)/HFIP/60 }{ }^{\circ} \mathrm{C} / 10 \mathrm{~h}\end{array}$ & $<5^{b}$ \\
\hline 9 & $\begin{array}{l}{\left[\mathrm{Cp}^{*} \mathrm{IrCl}_{2}\right]_{2}(3 \mathrm{~mol} \%) / \mathrm{AgBF}_{4}(12 \mathrm{~mol} \%) / \mathrm{Boc}-\mathrm{Leu}-\mathrm{OH}(0.2} \\
\text { equiv)/HFIP/60 }{ }^{\circ} \mathrm{C} / 10 \mathrm{~h}\end{array}$ & 43 \\
\hline 10 & $\begin{array}{l}{\left[\mathrm{Cp}^{*} \mathrm{IrCl}_{2}\right]_{2}(3 \mathrm{~mol} \%) / \mathrm{AgBF}_{4}(12 \mathrm{~mol} \%) / \mathrm{Boc}-\mathrm{Val}-\mathrm{OH}(0.2} \\
\text { equiv)/HFIP/60 }{ }^{\circ} \mathrm{C} / 10 \mathrm{~h}\end{array}$ & 43 \\
\hline 11 & $\begin{array}{l}{\left[\mathrm{Cp}^{*} \mathrm{IrCl}_{2}\right]_{2}(3 \mathrm{~mol} \%) / \mathrm{AgBF}_{4}(12 \mathrm{~mol} \%) / \mathrm{Ac}-\mathrm{Leu}-\mathrm{OH}(0.2} \\
\text { equiv }) / \mathrm{HFIP} / 60^{\circ} \mathrm{C} / 10 \mathrm{~h}\end{array}$ & 51 \\
\hline 12 & $\begin{array}{l}{\left[\mathrm{Cp}^{*} \mathrm{IrCl}_{2}\right]_{2}(3 \mathrm{~mol} \%) / \mathrm{AgBF}_{4}(12 \mathrm{~mol} \%) / \mathrm{Boc}-\mathrm{Pro}-\mathrm{OH}(0.2} \\
\text { equiv }) / \mathrm{HFIP} / 60^{\circ} \mathrm{C} / 6 \mathrm{~h}\end{array}$ & 77 \\
\hline 13 & $\begin{array}{l}{\left[\mathrm{Cp}^{*} \mathrm{IrCl}_{2}\right]_{2}(3 \mathrm{~mol} \%) / \mathrm{AgBF}_{4}(12 \mathrm{~mol} \%) / \mathrm{Boc}-\mathrm{Gly}-\mathrm{OH}(0.2} \\
\text { equiv)/HFIP/60 }{ }^{\circ} \mathrm{C} / 6 \mathrm{~h}\end{array}$ & 30 \\
\hline 14 & $\mathrm{AgBF}_{4}(12 \mathrm{~mol} \%) / \mathrm{Boc}-\mathrm{Pro}-\mathrm{OH}(20 \mathrm{~mol} \%) / \mathrm{HFIP} / 60^{\circ} \mathrm{C} / 6 \mathrm{~h}$ & NR \\
\hline 15 & {$\left[\mathrm{Cp} * \mathrm{IrCl}_{2}\right]_{2}(3 \mathrm{~mol} \%) / \mathrm{AgBF}_{4}(12 \mathrm{~mol} \%) / \mathrm{HFIP} / 60^{\circ} \mathrm{C} / 6 \mathrm{~h}$} & NR \\
\hline 16 & $\begin{array}{l}{\left[\mathrm{Cp}^{*} \mathrm{IrCl}_{2}\right]_{2}(3 \mathrm{~mol} \%) / \mathrm{AgBF}_{4}(12 \mathrm{~mol} \%) / \mathrm{Boc}-\mathrm{Pro}-\mathrm{OH}(0.2} \\
\text { equiv)/HFIP } / 40^{\circ} \mathrm{C} / 6 \mathrm{~h}\end{array}$ & 45 \\
\hline 17 & $\begin{array}{l}{\left[\mathrm{Cp}^{*} \mathrm{IrCl}_{2}\right]_{2}(3 \mathrm{~mol} \%) / \mathrm{AgBF}_{4}(12 \mathrm{~mol} \%) / \mathrm{Boc}-\mathrm{Pro}-\mathrm{OH}(0.2} \\
\text { equiv)/HFIP/rt/ } 10 \mathrm{~h}\end{array}$ & 38 \\
\hline 18 & $\begin{array}{l}{\left[\mathrm{Cp}^{*} \mathrm{IrCl}_{2}\right]_{2}(3 \mathrm{~mol} \%) / \mathrm{AgBF}_{4}(12 \mathrm{~mol} \%) / \mathrm{Boc}-\mathrm{Pro}-\mathrm{OH}(0.2} \\
\text { equiv)/DCE} / 60^{\circ} \mathrm{C} / 6 \mathrm{~h}\end{array}$ & NR \\
\hline 19 & $\begin{array}{l}{\left[\mathrm{Cp}^{*} \mathrm{IrCl}_{2}\right]_{2}(3 \mathrm{~mol} \%) / \mathrm{AgBF}_{4}(12 \mathrm{~mol} \%) / \text { Boc-Pro-OH }(0.2 \text { equiv })} \\
/ 1,4-\text { dioxane } / 60^{\circ} \mathrm{C} / 6 \mathrm{~h}\end{array}$ & $\begin{array}{l}\text { Complex } \\
\text { mixture }\end{array}$ \\
\hline 20 & $\begin{array}{l}{\left[\mathrm{Cp}^{*} \mathrm{IrCl}_{2}\right]_{2}(3 \mathrm{~mol} \%) / \mathrm{AgBF}_{4}(12 \mathrm{~mol} \%) / \mathrm{Boc}-\mathrm{Pro}-\mathrm{OH}(0.2} \\
\text { equiv)/toluene } / 60^{\circ} \mathrm{C} / 6 \mathrm{~h}\end{array}$ & NR \\
\hline 21 & $\begin{array}{l}{\left[\mathrm{Ru}(p \text {-cym }) \mathrm{Cl}_{2}\right]_{2}(3 \mathrm{~mol} \%) / \mathrm{AgBF}_{4}(12 \mathrm{~mol} \%) / \mathrm{Boc}-\mathrm{Pro}-\mathrm{OH}(0.2} \\
\text { equiv }) / \mathrm{HFIP} / 60^{\circ} \mathrm{C} / 6 \mathrm{~h}\end{array}$ & NR \\
\hline 22 & $\begin{array}{l}\mathrm{Cp}^{*} \mathrm{Co}(\mathrm{CO}) \mathrm{I}_{2}(5 \mathrm{~mol} \%) / \mathrm{AgBF}_{4}(12 \mathrm{~mol} \%) / \mathrm{Boc}-\mathrm{Pro}-\mathrm{OH}(0.2 \\
\text { equiv)/HFIP/ } 60^{\circ} \mathrm{C} / 6 \mathrm{~h}\end{array}$ & NR \\
\hline
\end{tabular}

${ }^{a}$ Isolated yields after column chromatography. NR $=$ No Reaction.

Table S2: Optimization of reaction conditions for $\alpha$-diazo- $\beta$-ketoesters:

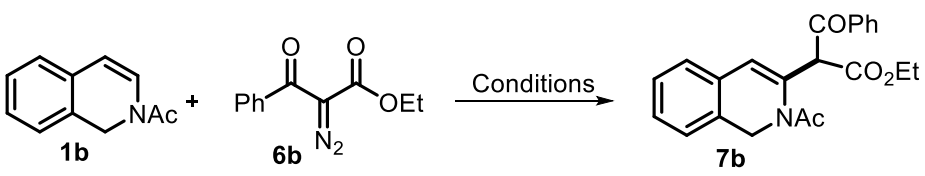




\begin{tabular}{|c|c|c|}
\hline Entry & Conditions & $\begin{array}{l}\text { Yield of } 7 \mathbf{b} \\
(\%)^{a}\end{array}$ \\
\hline 1 & $\begin{array}{l}{\left[\mathrm{Cp}^{*} \mathrm{RhCl}_{2}\right]_{2}(3 \mathrm{~mol} \%) / \mathrm{AgSbF}_{6}(9 \mathrm{~mol} \%) / \mathrm{PivOH}(0.5 \text { equiv) } \mathrm{DCE} / 40} \\
{ }^{\circ} \mathrm{C} / 6 \mathrm{~h}\end{array}$ & 41 \\
\hline 2 & $\begin{array}{l}{\left[\mathrm{Cp}^{*} \mathrm{RhCl}_{2}\right]_{2}(3 \mathrm{~mol} \%) / \mathrm{AgSbF}_{6}(9 \mathrm{~mol} \%) / \mathrm{PivOH}(1.0 \text { equiv }) / \mathrm{DCE} / 40} \\
{ }^{\circ} \mathrm{C} / 4 \mathrm{~h}\end{array}$ & 74 \\
\hline 3 & $\begin{array}{l}{\left[\mathrm{Cp}^{*} \mathrm{RhCl}_{2}\right]_{2}(3 \mathrm{~mol} \%) / \mathrm{AgSbF}_{6}(9 \mathrm{~mol} \%) / \mathrm{PivOH}(1.0 \text { equiv }) /} \\
\mathrm{DCE} / 25^{\circ} \mathrm{C} / 8 \mathrm{~h}\end{array}$ & 69 \\
\hline 4 & $\begin{array}{l}{\left[\mathrm{Cp}^{*} \mathrm{RhCl}_{2}\right]_{2}(3 \mathrm{~mol} \%) / \mathrm{AgSbF}_{6}(9 \mathrm{~mol} \%) / \mathrm{PivOH}(0.5 \text { equiv }) /} \\
\mathrm{AgOAc}(1.0 \text { equiv }) / \mathrm{DCE} / 40^{\circ} \mathrm{C} / 6 \mathrm{~h}\end{array}$ & 33 \\
\hline 5 & $\begin{array}{l}{\left[\mathrm{Cp}^{*} \mathrm{RhCl}_{2}\right]_{2}(1 \mathrm{~mol} \%) / \mathrm{AgSbF}_{6}(3 \mathrm{~mol} \%) / \mathrm{PivOH}(1.0 \text { equiv }) /} \\
\mathrm{DCE} / 40^{\circ} \mathrm{C} / 6 \mathrm{~h}\end{array}$ & 77 \\
\hline 6 & $\begin{array}{l}{\left[\mathrm{Cp}^{*} \mathrm{RhCl}_{2}\right]_{2}(3 \mathrm{~mol} \%) / \mathrm{AgSbF}_{6}(9 \mathrm{~mol} \%) / \mathrm{PivOH}(1.0 \text { equiv)/ THF } / 60} \\
{ }^{\circ} \mathrm{C} / 6 \mathrm{~h}\end{array}$ & Trace \\
\hline 7 & $\begin{array}{l}{\left[\mathrm{Cp}^{*} \mathrm{RhCl}_{2}\right]_{2}(3 \mathrm{~mol} \%) / \mathrm{AgSbF}_{6}(9 \mathrm{~mol} \%) / \mathrm{PivOH}(1.0 \text { equiv }) / \mathrm{DCM} / 50} \\
{ }^{\circ} \mathrm{C} / 6 \mathrm{~h}\end{array}$ & 30 \\
\hline 8 & $\begin{array}{l}{\left[\mathrm{Cp}^{*} \mathrm{RhCl}_{2}\right]_{2}(3 \mathrm{~mol} \%) / \mathrm{AgSbF}_{6}(9 \mathrm{~mol} \%) / \mathrm{PivOH}(1.0 \text { equiv }) / \mathrm{EtOH} / 40} \\
{ }^{\circ} \mathrm{C} / 6 \mathrm{~h}\end{array}$ & 15 \\
\hline 9 & $\begin{array}{l}{\left[\mathrm{Cp}^{*} \mathrm{RhCl}_{2}\right]_{2}(3 \mathrm{~mol} \%) / \mathrm{AgSbF}_{6}(9 \mathrm{~mol} \%) / \mathrm{PivOH}(1.0 \text { equiv }) /} \\
\text { toluene } / 110^{\circ} \mathrm{C} / 6 \mathrm{~h}\end{array}$ & NR \\
\hline 10 & $\begin{array}{l}{\left[\mathrm{Cp}^{*} \mathrm{RhCl}_{2}\right]_{2}(3 \mathrm{~mol} \%) / \mathrm{AgSbF}_{6}(9 \mathrm{~mol} \%) / \mathrm{PivOH}(1.0 \text { equiv }) / \mathrm{MeCN}} \\
/ 60^{\circ} \mathrm{C} / 6 \mathrm{~h}\end{array}$ & Trace \\
\hline 11 & $\begin{array}{l}{\left[\mathrm{Cp}^{*} \mathrm{RhCl}_{2}\right]_{2}(3 \mathrm{~mol} \%) / \mathrm{AgSbF}_{6}(9 \mathrm{~mol} \%) / \mathrm{PivOH}(1.0 \text { equiv }) / 1,4-} \\
\text { dioxane } / 100^{\circ} \mathrm{C} / 6 \mathrm{~h}\end{array}$ & NR \\
\hline 12 & $\begin{array}{l}{\left[\mathrm{Cp}^{*} \mathrm{RhCl}_{2}\right]_{2}(3 \mathrm{~mol} \%) / \mathrm{AgSbF}_{6}(9 \mathrm{~mol} \%) / \mathrm{PivOH} \text { (1.0 equiv)/ TFE} / 70} \\
{ }^{\circ} \mathrm{C} / 6 \mathrm{~h}\end{array}$ & 26 \\
\hline 13 & $\begin{array}{l}{\left[\mathrm{Cp}^{*} \mathrm{RhCl}_{2}\right]_{2}(3 \mathrm{~mol} \%) / \mathrm{AgSbF}_{6}(9 \mathrm{~mol} \%) / \mathrm{PivOH}(1.0 \text { equiv)/ }} \\
\mathrm{PhCF}_{3} / 100^{\circ} \mathrm{C} / 6 \mathrm{~h}\end{array}$ & $\begin{array}{l}\text { Complex } \\
\text { mixture }\end{array}$ \\
\hline 14 & {$\left[\mathrm{Cp}^{*} \mathrm{RhCl}_{2}\right]_{2}(3 \mathrm{~mol} \%) / \mathrm{AgOAc}\left(1.0\right.$ equiv)/DCE$/ 60^{\circ} \mathrm{C} / 6 \mathrm{~h}$} & NR \\
\hline 15 & $\mathrm{AgSbF}_{6}(9 \mathrm{~mol} \%) / \mathrm{PivOH}\left(1.0\right.$ equiv)/DCE/ $40^{\circ} \mathrm{C} / 6 \mathrm{~h}$ & NR \\
\hline 16 & {$\left[\mathrm{Cp}^{*} \mathrm{RhCl}_{2}\right]_{2}(3 \mathrm{~mol} \%) / \mathrm{PivOH}\left(1.0\right.$ equiv)/DCE$/ 40{ }^{\circ} \mathrm{C} / 6 \mathrm{~h}$} & NR \\
\hline 17 & {$\left[\mathrm{Cp}^{*} \mathrm{RhCl}_{2}\right]_{2}(3 \mathrm{~mol} \%) / \mathrm{AgSbF}_{6}(9 \mathrm{~mol} \%) / \mathrm{DCE} / 40{ }^{\circ} \mathrm{C} / 6 \mathrm{~h}$} & 11 \\
\hline 18 & $\begin{array}{l}{\left[\mathrm{Cp}^{*} \mathrm{RhCl}_{2}\right]_{2}(3 \mathrm{~mol} \%) / \mathrm{AgSbF}_{6}(9 \mathrm{~mol} \%) / \mathrm{AcOH}(50 \mathrm{~mol} \%) / \mathrm{DCE} / 40} \\
{ }^{\circ} \mathrm{C} / 6 \mathrm{~h}\end{array}$ & Trace \\
\hline 19 & {$\left[\mathrm{Cp}^{*} \mathrm{RhCl}_{2}\right]_{2}(3 \mathrm{~mol} \%) / \mathrm{AcOH}(1.0$ equiv $) / \mathrm{DCE} / 60^{\circ} \mathrm{C} / 4 \mathrm{~h}$} & $\begin{array}{l}\text { Complex } \\
\text { mixture }\end{array}$ \\
\hline 20 & $\begin{array}{l}{\left[\mathrm{Cp}^{*} \mathrm{RhCl}_{2}\right]_{2}(3 \mathrm{~mol} \%) / \mathrm{AgOTf}(10 \mathrm{~mol} \%) / \mathrm{PivOH}(1.0 \text { equiv)/DCE/60 }} \\
{ }^{\circ} \mathrm{C} / 6 \mathrm{~h}\end{array}$ & Trace \\
\hline
\end{tabular}




\begin{tabular}{|c|c|c|}
\hline 21 & {$\left[\mathrm{Ru}(p-\mathrm{cym}) \mathrm{Cl}_{2}\right]_{2}(3 \mathrm{~mol} \%) / \mathrm{AgSbF}_{6}(9 \mathrm{~mol} \%) / \mathrm{DCE} / 70{ }^{\circ} \mathrm{C} / 12 \mathrm{~h}$} & NR \\
\hline 22 & $\begin{array}{l}{\left[\mathrm{Ru}(p-\mathrm{cym}) \mathrm{Cl}_{2}\right]_{2}(3 \mathrm{~mol} \%) / \mathrm{AgSbF}_{6}(9 \mathrm{~mol} \%) / \mathrm{PivOH}(1.0 \text { equiv }) /} \\
\mathrm{DCE} / 70^{\circ} \mathrm{C} / 12 \mathrm{~h}\end{array}$ & NR \\
\hline 23 & $\begin{array}{l}{\left[\mathrm{Ru}(p-\mathrm{cym}) \mathrm{Cl}_{2}\right]_{2}(3 \mathrm{~mol} \%) / \mathrm{AgSbF}_{6}(9 \mathrm{~mol} \%) / \mathrm{PivOH}(1.0 \text { equiv }) /} \\
\mathrm{MeOH} / 60{ }^{\circ} \mathrm{C} / 6 \mathrm{~h}\end{array}$ & NR \\
\hline 24 & $\begin{array}{l}{\left[\mathrm{Ru}(p-\mathrm{cym}) \mathrm{Cl}_{2}\right]_{2}(3 \mathrm{~mol} \%) / \mathrm{AgSbF}_{6}(9 \mathrm{~mol} \%) / \mathrm{PivOH}(1.0 \text { equiv }) /} \\
\text { toluene } / 100{ }^{\circ} \mathrm{C} / 6 \mathrm{~h}\end{array}$ & NR \\
\hline 25 & $\begin{array}{l}{\left[\mathrm{Ru}(p-\mathrm{cym}) \mathrm{Cl}_{2}\right]_{2}(3 \mathrm{~mol} \%) / \mathrm{AgSbF}_{6}(9 \mathrm{~mol} \%) / \mathrm{CsOAc}(1.0} \\
\text { equiv }) /(\mathrm{PhO})_{2} \mathrm{P}(\mathrm{O}) \mathrm{OH}(1.5 \text { equiv }) / \mathrm{DCE} / 80^{\circ} \mathrm{C} / 12 \mathrm{~h}\end{array}$ & NR \\
\hline 26 & $\begin{array}{l}\mathrm{Cp}^{*} \mathrm{Co}(\mathrm{CO}) \mathrm{I}_{2}(5 \mathrm{~mol} \%) / \mathrm{AgSbF}_{6}(10 \mathrm{~mol} \%) / \mathrm{AgOAc}(1.0 \text { equiv }) / \\
\mathrm{DCE} / 80^{\circ} \mathrm{C} / 12 \mathrm{~h}\end{array}$ & NR \\
\hline 27 & $\begin{array}{l}\mathrm{Cp}^{*} \mathrm{Co}(\mathrm{CO}) \mathrm{I}_{2}(5 \mathrm{~mol} \%) / \mathrm{AgSbF}_{6}(10 \mathrm{~mol} \%) / \mathrm{PivOH}(1.0 \text { equiv }) / \\
\mathrm{DCE} / 80^{\circ} \mathrm{C} / 6 \mathrm{~h}\end{array}$ & $\begin{array}{l}\text { Complex } \\
\text { mixture }\end{array}$ \\
\hline 28 & $\begin{array}{l}\mathrm{Cp}^{*} \mathrm{Co}(\mathrm{CO}) \mathrm{I}_{2}(5 \mathrm{~mol} \%) / \mathrm{AgSbF}_{6}(10 \mathrm{~mol} \%) / \mathrm{PivOH}(1.0 \text { equiv }) / \\
\text { toluene } / 100{ }^{\circ} \mathrm{C} / 12 \mathrm{~h}\end{array}$ & NR \\
\hline 29 & $\mathrm{Cp} * \mathrm{Co}(\mathrm{CO}) \mathrm{I}_{2}(5 \mathrm{~mol} \%) /$ toluene $/ 100^{\circ} \mathrm{C} / 12 \mathrm{~h}$ & NR \\
\hline 30 & $\begin{array}{l}\mathrm{Co}(\mathrm{acac})_{2}(5 \mathrm{~mol} \%) / \mathrm{AgSbF}_{6}(10 \mathrm{~mol} \%) / \mathrm{PivOH}(1.0 \text { equiv }) / \mathrm{DCE} / 80 \\
{ }^{\circ} \mathrm{C} / 12 \mathrm{~h}\end{array}$ & NR \\
\hline 31 & $\begin{array}{l}{\left[\mathrm{Cp}^{*} \mathrm{IrCl}_{2}\right]_{2}(3 \mathrm{~mol} \%) / \mathrm{AgBF}_{4}(12 \mathrm{~mol} \%) / \mathrm{Boc}-\mathrm{Pro}-\mathrm{OH}(0.2} \\
\text { equiv }) / \mathrm{HFIP} / 60^{\circ} \mathrm{C} / 6 \mathrm{~h}\end{array}$ & $\begin{array}{l}\text { Complex } \\
\text { mixture }\end{array}$ \\
\hline 32 & $\begin{array}{l}{\left[\mathrm{Cp}^{*} \mathrm{IrCl}_{2}\right]_{2}(3 \mathrm{~mol} \%) / \mathrm{AgBF}_{4}(12 \mathrm{~mol} \%) / \mathrm{Ac}-\mathrm{Gly}-\mathrm{OH}(0.2} \\
\text { equiv)/HFIP } / 60^{\circ} \mathrm{C} / 6 \mathrm{~h}\end{array}$ & 51 \\
\hline 33 & $\begin{array}{l}{\left[\mathrm{Cp}^{*} \mathrm{IrCl}_{2}\right]_{2}(3 \mathrm{~mol} \%) / \mathrm{AgBF}_{4}(12 \mathrm{~mol} \%) / \text { Boc-Leu-OH }(0.2} \\
\text { equiv }) / \mathrm{HFIP} / 60^{\circ} \mathrm{C} / 6 \mathrm{~h}\end{array}$ & 77 \\
\hline
\end{tabular}

\section{iv) General procedure for the $\operatorname{Ir}(\mathrm{III})$-catalyzed $\mathrm{C}(3)$-alkylation.}

In a pressure tube equipped with a stir bar, $N$-acetyl-1,2-dihydroisoquinolines $1(0.1 \mathrm{mmol})$, diazo compound (0.12 mmol), [Cp* $\left.\mathrm{IrCl}_{2}\right]_{2}(2.4 \mathrm{mg}, 3 \mathrm{~mol} \%), \mathrm{AgBF}_{4}(2.4 \mathrm{mg}, 12 \mathrm{~mol} \%)$ and Boc-Pro-OH (4.3 mg, 0.2 equiv) were added simultaneously under argon flow. This was followed by the addition of distilled HFIP $(1.0 \mathrm{~mL})$. The reaction tube was sealed and immersed in an oil bath preheated to $60^{\circ} \mathrm{C}$, with stirring. Thereafter, progress of the reaction was checked by TLC. Upon completion, the reaction mixture was cooled to room temperature, filtered through a pad of Celite and eluted with EtOAc. The filtrate was washed with brine, and dried over anhydrous $\mathrm{Na}_{2} \mathrm{SO}_{4}$. After concentration of the organic extract under reduced pressure, the crude product was purified by a silica gel flash column chromatography to yield the desired C(3)-alkylated product. 


\section{Analytical data:}

Dimethyl-2-(2-acetyl-1-phenyl-1,2-dihydroisoquinolin-3-yl)malonate (mixture of rotamers)<smiles>CC(=O)C(C(C)=O)C1=Cc2ccccc2C(c2ccccc2)N1</smiles>

(3a): Yield: 86\% (33 mg), Physical appearance: yellow solid; M.p. 115-116

${ }^{\circ} \mathrm{C}$; TLC $R_{f} 0.4$ (1:1, Petroleum ether: EtOAc); ${ }^{1} \mathbf{H}$ NMR $\left(500 \mathrm{MHz}, \mathrm{CDCl}_{3}\right)$ $\delta 7.40-7.31(\mathrm{~m}, 6 \mathrm{H}), 7.30-7.24(\mathrm{~m}, 8 \mathrm{H}), 7.15-7.06(\mathrm{~m}, 3 \mathrm{H}), 6.61(\mathrm{~s}, 1 \mathrm{H})$, $6.06(\mathrm{~s}, 1 \mathrm{H}), 4.64(\mathrm{~s}, 1 \mathrm{H}), 3.83(\mathrm{~s}, 6 \mathrm{H}), 3.40(\mathrm{~s}, 5 \mathrm{H}), 2.44(\mathrm{~s}, 4 \mathrm{H}) ;{ }^{13} \mathbf{C} \mathbf{N M R}\left(176 \mathrm{MHz}, \mathrm{CDCl}_{3}\right)$ $\delta 170.3,168.0,167.3,138.8,132.3,130.8,130.5,128.4,128.2,127.9,127.7,126.7,126.5,126.4$, 120.7, 60.9, 55.5, 52.8, 52.7, 22.4; IR( $\left.\mathrm{cm}^{-1}\right):$ 2954, 177431712, 1651, 1448, 1400, 1329, 1260, 1179; HRMS (ESI) $m / z:[\mathrm{M}+\mathrm{Na}]^{+}$calcd. for $\mathrm{C}_{22} \mathrm{H}_{21} \mathrm{NO}_{5} \mathrm{Na} 402.1312$; found 402.1294 .

Dimethyl 2-(2-acetyl-1,2-dihydroisoquinolin-3-yl)malonate (3b): Yield: $77 \%$ (23 mg),

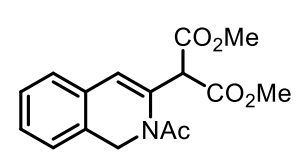

Physical appearance: pale-yellow solid, M. p. 96-98 ${ }^{\circ}$ C, TLC $R_{f} 0.40$ (1:1, Petroleum ether: EtOAc); ${ }^{1} \mathbf{H}$ NMR $\left(500 \mathrm{MHz} \mathrm{CDCl}_{3}\right) \delta 7.28-7.15(\mathrm{~m}$, $3 \mathrm{H}), 7.12(\mathrm{~d}, \mathrm{~J}=7.3 \mathrm{~Hz}, 1 \mathrm{H}), 6.54(\mathrm{~s}, 1 \mathrm{H}), 4.94$ (s, 1H), 4.64 (s, 2H), 3.79 (s, 6H), $2.23(\mathrm{~s}, 3 \mathrm{H}) ;{ }^{13} \mathrm{C}$ NMR (176 MHz, $\left.\mathrm{CDCl}_{3}\right) \delta 169.8,167.9,133.8,131.3,131.0,130.9$, 128.1, 128.0, 127.9, 125.7, 124.4, 120.3, 55.1, 52.9, 49.9, 22.6; IR $\left(\mathrm{cm}^{-1}\right): 2954,1732,1654$, 1621, 1504, 1269; HRMS (ESI) $m / z:[\mathrm{M}+\mathrm{Na}]^{+}$calcd. for $\mathrm{C}_{16} \mathrm{H}_{17} \mathrm{NO}_{5} \mathrm{Na}$ 326.0999; found 326.1025 .

Dimethyl 2-(2-acetyl-1-methyl-1,2-dihydroisoquinolin-3-yl)malonate (3c): Yield: 68\% (22 ether: EtOAc); ${ }^{1} \mathbf{H}$ NMR $\left(500 \mathrm{MHz}, \mathrm{CDCl}_{3}\right) \delta 7.27-7.16(\mathrm{~m}, 2 \mathrm{H}), 7.07(\mathrm{~d}, \mathrm{~J}$
$=6.9 \mathrm{~Hz}, 1 \mathrm{H}), 6.52(\mathrm{~s}, 1 \mathrm{H}), 4.99(\mathrm{~s}, 1 \mathrm{H}), 4.76(\mathrm{~s}, 1 \mathrm{H}), 3.84(\mathrm{~s}, 2 \mathrm{H}), 3.76(\mathrm{~s}$, 2H), $2.27(\mathrm{~s}, 2 \mathrm{H}) ;{ }^{13} \mathbf{C} \mathbf{N M R}\left(176 \mathrm{MHz} \mathrm{CDCl}_{3}\right) \delta 169.7,168.4,167.5,139.6,139.5,135.7$, 135.6, 135.6, 129.9, 129.3, 128.5, 128.5, 128.0, 127.8, 126.2, 124.4, 119.5, 55.4, 54.9, 52.8, 22.4, 20.4; IR $\left(\mathrm{cm}^{-1}\right): 2975,1661,1623,1493,1455,1349,1327,1238 ;$ HRMS (ESI) $m / z:[\mathrm{M}+\mathrm{Na}]^{+}$ calcd. for $\mathrm{C}_{17} \mathrm{H}_{19} \mathrm{NO}_{5} \mathrm{Na} 340.1155$; found 340.1161 .

Dimethyl 2-(2-acetyl-1-isopropyl-1,2-dihydroisoquinolin-3-yl)malonate (3d): Yield: 67\% (23

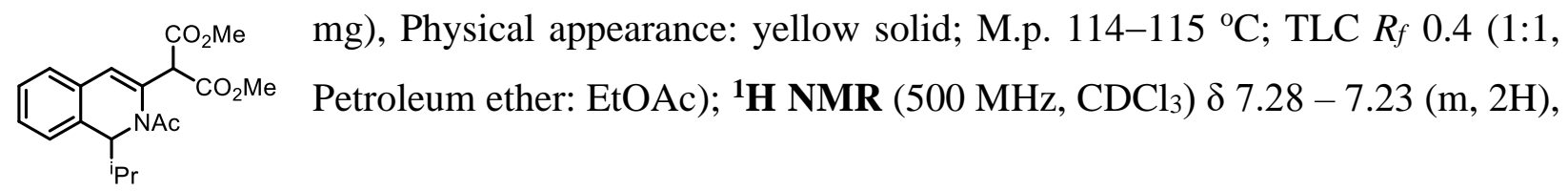


$7.23-7.17(\mathrm{~m}, 2 \mathrm{H}), 7.05(\mathrm{~d}, J=7.3 \mathrm{~Hz}, 1 \mathrm{H}), 6.59(\mathrm{~s}, 1 \mathrm{H}), 4.68(\mathrm{~s}, 1 \mathrm{H}), 4.34-4.19(\mathrm{~m}, 1 \mathrm{H})$, $3.83(\mathrm{~s}, 3 \mathrm{H}), 3.75(\mathrm{~s}, 3 \mathrm{H}), 2.23(\mathrm{~s}, 3 \mathrm{H}), 1.97(\mathrm{~s}, 1 \mathrm{H}), 1.05$ (d, J=6.5 Hz, 3H), 0.79 (d, J = 6.7 Hz, 3H); ${ }^{13} \mathbf{C}$ NMR $\left(176 \mathrm{MHz}, \mathrm{CDCl}_{3}\right) \delta 170.6,168.5,167.5,133.2,130.8,129.9,127.8,127.2$, 126.5, 126.2, 120.6, 65.8, 55.7, 52.9, 52.8, 29.7, 29.5, 22.5, 19.7, 19.5; IR $\left(\mathrm{cm}^{-1}\right): 2953,1765$, 1735, 1627, 1641, 1405, 1295, 1255, 1174; HRMS (ESI) $m / z:[\mathrm{M}+\mathrm{Na}]^{+}$calcd. for $\mathrm{C}_{19} \mathrm{H}_{23} \mathrm{NO}_{5} \mathrm{Na} 368.1468$; found 368.1495.

\section{Dimethyl-2-(2-acetyl-1-ethyl-1,2-dihydroisoquinolin-3-yl)malonate (3e):}

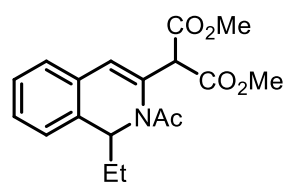

Yield: 65\% (21 mg), Physical appearance: yellow solid; M.p. 95-96 ${ }^{\circ}$ C; TLC $R_{f} 0.4$ (1:1, Petroleum ether: EtOAc); ${ }^{1} \mathbf{H}$ NMR (400 $\left.\mathrm{MHz}, \mathrm{CDCl}_{3}\right) \delta 7.26$ $(\mathrm{dd}, J=7.5,1.4 \mathrm{~Hz}, 1 \mathrm{H}), 7.23(\mathrm{~d}, J=2.1 \mathrm{~Hz}, 1 \mathrm{H}), 7.22-7.18(\mathrm{~m}, 2 \mathrm{H}), 7.07$ $(\mathrm{d}, J=6.4 \mathrm{~Hz}, 1 \mathrm{H}), 6.56(\mathrm{~s}, 1 \mathrm{H}), 4.71(\mathrm{~s}, 2 \mathrm{H}), 3.84(\mathrm{~s}, 3 \mathrm{H}), 3.75(\mathrm{~s}, 3 \mathrm{H}), 2.27$ (d, $J=10.8 \mathrm{~Hz}$, 3H), 1.78 (br s, 1H), $1.62-1.55(\mathrm{~m}, 1 \mathrm{H}), 0.96(\mathrm{t}, J=7.4 \mathrm{~Hz}, 3 \mathrm{H}) ;{ }^{13} \mathbf{C} \mathbf{N M R}\left(176 \mathrm{MHz}, \mathrm{CDCl}_{3}\right)$ $\delta 170.5,168.5,167.5,135.7,134.8,130.3,129.6,128.6,127.8,127.7,126.3,125.0,120.1,60.9$, 55.5, 52.8, 26.8, 22.5, 21.1, 20.1, 10.5; IR (cm $\left.{ }^{-1}\right): 2922,2852,1731,1651,1640,1403,1286$, 1286; HRMS (ESI) $m / z:[\mathrm{M}+\mathrm{Na}]^{+}$calcd. for $\mathrm{C}_{18} \mathrm{H}_{21} \mathrm{NO}_{5} \mathrm{Na} 354.1312$; found 354.1315.

\section{Dimethyl-2-(2-acetyl-1-butyl-1,2-dihydroisoquinolin-3-yl)malonate (3f):}

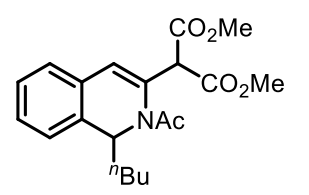

Yield: 40\% (14 mg), Physical appearance: yellow solid; M.p. $122-124{ }^{\circ} \mathrm{C}$;

TLC $R_{f} 0.4$ (1:1, Petroleum ether: EtOAc); ${ }^{1} \mathbf{H}$ NMR (400 $\left.\mathrm{MHz} \mathrm{CDCl}_{3}\right) \delta$ $7.25-7.15(\mathrm{~m}, 3 \mathrm{H}), 7.06(\mathrm{~d}, J=6.1 \mathrm{~Hz}, 1 \mathrm{H}), 6.56(\mathrm{~s}, 1 \mathrm{H}), 4.76(\mathrm{~s}, 1 \mathrm{H}), 4.67$ (s, 1H), $3.84(\mathrm{~s}, 3 \mathrm{H}), 3.75(\mathrm{~s}, 3 \mathrm{H}), 2.24(\mathrm{~s}, 3 \mathrm{H}), 1.76(\mathrm{~s}, 1 \mathrm{H}), 1.50(\mathrm{~d}, J=13.2 \mathrm{~Hz}, 1 \mathrm{H}), 1.31-1.28$ $(\mathrm{m}, 4 \mathrm{H}), 0.90(\mathrm{t}, J=6.9 \mathrm{~Hz}, 3 \mathrm{H}) ;{ }^{13} \mathbf{C} \mathbf{N M R}\left(176 \mathrm{MHz}, \mathrm{CDCl}_{3}\right) \delta$ 170.4, 168.5, 167.5, 135.1, $130.3,129.6,127.8,126.3,124.9,120.2,59.5,55.6,52.8,33.6,28.2,22.5,14.0,13.9$; IR $\left(\mathrm{cm}^{-1}\right)$ : 2955, 2933, 2853, 1758, 1731, 1654, 1406, 1289, 1142; HRMS (ESI) $m / z:[\mathrm{M}+\mathrm{Na}]^{+}$calcd. for $\mathrm{C}_{20} \mathrm{H}_{25} \mathrm{NO}_{5} \mathrm{Na} 382.1625$; found 382.1642.

\section{Diethyl-2-(2-acetyl-1, 2-dihydroisoquinolin-3-yl)malonate (3h):}

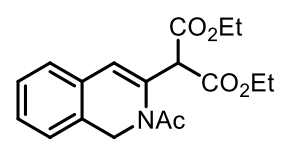

Yield: 71\% (23 mg); Physical appearance: yellow gel; TLC $R_{f} 0.4$ (1:1,

Petroleum ether: EtOAc); ${ }^{\mathbf{1}} \mathbf{H}$ NMR $\left(500 \mathrm{MHz} \mathrm{CDCl}_{3}\right) \delta 7.27-7.22(\mathrm{~m}, 1 \mathrm{H})$, $7.22-7.18(\mathrm{~m}, 1 \mathrm{H}), 7.17(\mathrm{dd}, \mathrm{J}=7.2,1.5 \mathrm{~Hz}, 1 \mathrm{H}), 7.12(\mathrm{~d}, \mathrm{~J}=7.4 \mathrm{~Hz}, 1 \mathrm{H})$, $6.56(\mathrm{~s}, 1 \mathrm{H}), 4.94(\mathrm{~s}, 1 \mathrm{H}), 4.63(\mathrm{~s}, 2 \mathrm{H}), 4.33-4.18(\mathrm{~m}, 4 \mathrm{H}), 2.23(\mathrm{~s}, 3 \mathrm{H}), 1.29(\mathrm{t}, \mathrm{J}=7.1 \mathrm{~Hz}$, 6H); ${ }^{13} \mathrm{C}$ NMR $\left(176 \mathrm{MHz}, \mathrm{CDCl}_{3}\right) \delta 169.7,167.5,134.2,131.4,130.9,128.1,127.8,125.7$, 
124.4, 120.1, 61.8, 55.4, 50.0, 22.6, 14.0; IR $\left(\mathrm{cm}^{-1}\right): 2945,1734,1260,1032$; HRMS (ESI) $\mathrm{m} / z$ : $[\mathrm{M}+\mathrm{H}]^{+}$calcd. for $\mathrm{C}_{18} \mathrm{H}_{22} \mathrm{NO}_{5} 332.1492$; found 332.1500.

Diisopropyl-2-(2-acetyl-1, 2-dihydroisoquinolin-3-yl)malonate (3i):

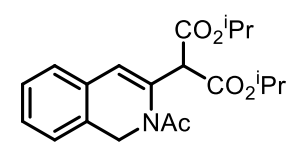

Yield: 70\% (25 mg); Physical appearance: yellow gel; TLC $R_{f} 0.4$ (1:1,

Petroleum ether: EtOAc); ${ }^{1} \mathbf{H}$ NMR $\left(500 \mathrm{MHz}, \mathrm{CDCl}_{3}\right) \delta 7.24$ (dd, $J=7.4,1.5$ $\mathrm{Hz}, 1 \mathrm{H}), 7.22-7.18(\mathrm{~m}, 1 \mathrm{H}), 7.17(\mathrm{dd}, J=7.3,1.5 \mathrm{~Hz}, 1 \mathrm{H}), 7.12(\mathrm{~d}, J=7.4$ $\mathrm{Hz}, 1 \mathrm{H}), 6.57$ (s, 1H), 5.17- $5.05(\mathrm{~m}, 2 \mathrm{H}), 4.90(\mathrm{~s}, 1 \mathrm{H}), 4.63(\mathrm{~s}, 2 \mathrm{H}), 2.23(\mathrm{~s}, 3 \mathrm{H}), 1.29$ (d, $J=$ $2.4 \mathrm{~Hz}, 6 \mathrm{H}), 1.27(\mathrm{~d}, J=2.4 \mathrm{~Hz}, 6 \mathrm{H}) ;{ }^{13} \mathrm{C} \mathbf{N M R}\left(126 \mathrm{MHz}, \mathrm{CDCl}_{3}\right) \delta 169.6,167.0,134.5,131.5$, 131.0, 128.0, 127.7, 125.7, 124.4, 119.9, 69.3, 55.7, 50.0, 22.7, 22.6, 21.6, 21.5; IR (cm $\left.{ }^{-1}\right): 2982$, 2936, 1723, 1654, 1455, 1346, 1285, 1173; HRMS (ESI) $m / z:\left[\mathrm{M}+\mathrm{Na}^{+}\right.$calcd. for $\mathrm{C}_{20} \mathrm{H}_{25} \mathrm{NO}_{5} \mathrm{Na} 382.1625$; found 382.1643.

Dibenzyl-2-(2-acetyl-1,2-dihydroisoquinolin-3-yl)malonate $\quad(3 \mathbf{3 j}): \quad$ Yield: $45 \% \quad(20 \mathrm{mg})$; Physical appearance: pale-yellow solid; M.p. 84-85 ${ }^{\circ} \mathrm{C}$; TLC $R_{f} 0.4(1: 1$,
Petroleum ether: EtOAc); ${ }^{1} \mathbf{H}$ NMR $\left(500 \mathrm{MHz}, \mathrm{CDCl}_{3}\right) \delta 7.36-7.31(\mathrm{~m}$, $10 \mathrm{H}), 7.28-7.19(\mathrm{~m}, 3 \mathrm{H}), 7.11(\mathrm{~d}, J=7.2 \mathrm{~Hz}, 1 \mathrm{H}), 7.07$ (dd, $J=7.1,1.6 \mathrm{~Hz}, 1 \mathrm{H}), 6.45(\mathrm{~s}, 1 \mathrm{H})$, $5.28-5.19(\mathrm{~m}, 4 \mathrm{H}), 5.14(\mathrm{~s}, 1 \mathrm{H}), 4.58(\mathrm{~s}, 2 \mathrm{H}), 2.20(\mathrm{~s}, 3 \mathrm{H}) ;{ }^{13} \mathbf{C} \mathbf{N M R}\left(126 \mathrm{MHz}, \mathrm{CDCl}_{3}\right) \delta$ 169.7 , 167.2, 135.3, 133.8, 131.3, 130.9, 128.5, 128.3, 128.2, 128.1, 127.9, 125.7, 124.4, 120.4, 67.5, 55.4, 49.9, 22.6; IR ( $\left.\mathrm{cm}^{-1}\right): 2962,1771,1722,1647,1410,1223,1185$; HRMS (ESI) $m / z$ : $[\mathrm{M}+\mathrm{Na}]^{+}$calcd. for $\mathrm{C}_{28} \mathrm{H}_{25} \mathrm{NO}_{5} \mathrm{Na} 478.1625$; found 478.1637.

Dimethyl 2-(2-acetyl-5-(4-methoxyphenyl)-1,2-dihydroisoquinolin-3-yl)malonate (3l): Yield:

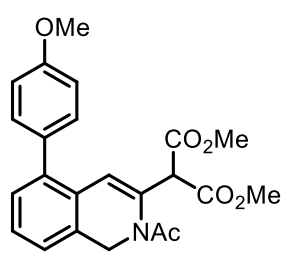
$67 \%(27 \mathrm{mg})$, Physical appearance: dark-yellow semi-solid, TLC $R_{f} 0.2$ (7:3, Petroleum ether: EtOAc); ${ }^{1} \mathbf{H}$ NMR $\left(400 \mathrm{MHz}^{\left.\mathrm{CDCl}_{3}\right)} \delta 7.38(\mathrm{~d}, J=8.1 \mathrm{~Hz}\right.$, $2 \mathrm{H}), 7.30(\mathrm{~d}, J=4.4 \mathrm{~Hz}, 2 \mathrm{H}), 7.13(\mathrm{t}, J=4.4 \mathrm{~Hz}, 1 \mathrm{H}), 7.00(\mathrm{~d}, J=8.6 \mathrm{~Hz}$, 2H), 6.54 (s, 1H), 5.08 (s, 1H), 4.69 (s, 2H), 3.88 (s, 3H), 3.77 (s, 6H), 2.29 (s, 3H); ${ }^{13} \mathrm{C}$ NMR $\left(126 \mathrm{MHz}, \mathrm{CDCl}_{3}\right) \delta 169.8,167.9,159.1,138.5,133.7,132.4,131.6,130.8$, 129.3, 128.8, 128.4, 127.7, 123.0, 119.6, 113.7, 55.3, 55.1, 52.8, 50.6, 22.5; IR $\left(\mathrm{cm}^{-1}\right): 2945$, 2850, 1763, 1715,1626, 1544, 1392, 1258; HRMS (ESI) $m / z:[\mathrm{M}+\mathrm{Na}]^{+}$calcd. for $\mathrm{C}_{23} \mathrm{H}_{23} \mathrm{NO}_{6} \mathrm{Na}$ 432.1438; found 432.1418 . 
Dimethyl-2-(2-acetyl-5-(4-fluorophenyl)-1,2-dihydroisoquinolin-3-yl)malonate (mixture of

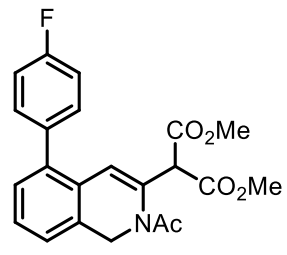
rotamers) (3m): Yield: 65\% (26 mg), Physical appearance: yellow semisolid, TLC $R_{f} 0.3$ (7:3, Petroleum ether: EtOAc); ${ }^{1} \mathbf{H}$ NMR (500 MHz, $\left.\mathrm{CDCl}_{3}\right) \delta 7.39(\mathrm{dd}, \mathrm{J}=8.4,5.4 \mathrm{~Hz}, 2 \mathrm{H}), 7.33-7.23(\mathrm{~m}, 2 \mathrm{H}), 7.18-7.09(\mathrm{~m}$, 3H), $6.46(\mathrm{~s}, 1 \mathrm{H}), 5.04(\mathrm{~s}, 1 \mathrm{H}), 4.68(\mathrm{~s}, 2 \mathrm{H}), 3.74(\mathrm{~s}, 6 \mathrm{H}) ;{ }^{13} \mathrm{C}$ NMR (126 $\mathrm{MHz}_{\mathrm{CDCl}} \mathrm{CD} \delta 169.9,168.1,167.9,162.4\left(\mathrm{~d}, J_{\mathrm{C}-\mathrm{F}}=247.3\right), 137.7,135.5,135.2,134.2,132.4$, $131.3\left(\mathrm{~d}, J_{\mathrm{C}-\mathrm{F}}=8.0\right), 129.3,128.9,127.8,126.8,125.8,123.6,120.4,119.2,115.1\left(\mathrm{~d}, J_{\mathrm{C}-\mathrm{F}}=\right.$ 21.6), 55.0, 52.8, 50.5, 22.5; ${ }^{19} \mathbf{F}$ NMR $\left(376 \mathrm{MHz}, \mathrm{CDCl}_{3}\right) \delta-114.87$; IR $\left(\mathrm{cm}^{-1}\right): 2995,2952$, 1754, 1731, 1658, 1399, 1220, 1207; HRMS (ESI) $m / z:[\mathrm{M}+\mathrm{Na}]^{+}$calcd. for $\mathrm{C}_{22} \mathrm{H}_{20} \mathrm{FNO}_{5} \mathrm{Na}$ 420.1218 ; found 420.1240 .

Dimethyl-2-(2-acetyl-5-bromo-1,2-dihydroisoquinolin-3-yl)malonate (3n): Yield: 52\% (20

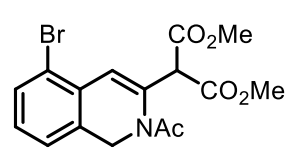

mg), Physical appearance: white solid, M.p. $112-113{ }^{\circ} \mathrm{C}$; TLC $R_{f} 0.3$ (7:3, Petroleum ether: EtOAc); ${ }^{1} \mathbf{H}$ NMR (500 $\left.\mathrm{MHz} \mathrm{CDCl}_{3}\right) \delta 7.50-7.42(\mathrm{~m}$, $1 \mathrm{H}), 7.11-7.03(\mathrm{~m}, 2 \mathrm{H}), 6.80(\mathrm{~s}, 1 \mathrm{H}), 5.05(\mathrm{~s}, 1 \mathrm{H}), 4.64(\mathrm{~s}, 2 \mathrm{H}), 3.81(\mathrm{~s}$, 6H), $2.23(\mathrm{~s}, 3 \mathrm{H}) ;{ }^{13} \mathrm{C}$ NMR (176 $\left.\mathrm{MHz}, \mathrm{CDCl}_{3}\right) \delta 169.7,167.7,135.7,132.9,132.2,131.1$, 128.9, 123.6, 121.4, 119.3, 55.2, 53.0, 50.3, 22.7; IR $\left(\mathrm{cm}^{-1}\right): 2951,1757,1733,1663,1584$, 1436, 1394, 1205; HRMS (ESI) $m / z:[\mathrm{M}+\mathrm{Na}]^{+}$calcd. for $\mathrm{C}_{16} \mathrm{H}_{16} \mathrm{BrNO}_{5} \mathrm{Na} 404.0104$ and 406.0084; found 404.0138 and 406.0084 .

Dimethyl 2-(2-acetyl-5-phenyl-1,2-dihydroisoquinolin-3-yl)malonate (mixture of rotamers)

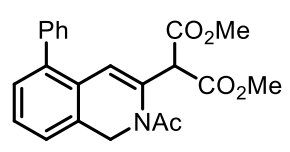

(3o): Yield: 64\% (24 mg); Physical appearance: yellow gel; TLC $R_{f} 0.3$ (7:3, Petroleum ether: EtOAc); ${ }^{1} \mathbf{H}$ NMR $\left(500 \mathrm{MHz}^{\mathrm{CDCl}} 3\right) \delta 7.46-7.39$ $(\mathrm{m}, 4 \mathrm{H}), 7.39-7.34(\mathrm{~m}, 1 \mathrm{H}), 7.32-7.28(\mathrm{~m}, 2 \mathrm{H}), 7.18-7.12(\mathrm{~m}, 1 \mathrm{H})$, $6.48(\mathrm{~s}, 1 \mathrm{H}), 5.10(\mathrm{~s}, 1 \mathrm{H}), 4.68(\mathrm{~s}, 2 \mathrm{H}), 3.73(\mathrm{~s}, 6 \mathrm{H}), 2.28(\mathrm{~s}, 3 \mathrm{H}) ;{ }^{13} \mathbf{C} \mathbf{N M R}\left(126 \mathrm{MHz}, \mathrm{CDCl}_{3}\right)$ $\delta 169.9,167.9,139.2,138.8,134.0,132.4,129.7,129.4,128.9,128.2,127.8,127.5,125.8,123.5$, 119.4, 55.1, 52.8, 50.6, 22.6; IR $\left(\mathrm{cm}^{-1}\right):$ 2952, 1735, 1655, 1434, 1398, 1259, 1216, 1148; HRMS (ESI) $m / z:[\mathrm{M}+\mathrm{Na}]^{+}$calcd. for $\mathrm{C}_{22} \mathrm{H}_{21} \mathrm{NO}_{5} \mathrm{Na} 402.1312$; found 402.1325 .

Dimethyl 2-(2-acetyl-6-phenyl-1,2-dihydroisoquinolin-3-yl)malonate (3p): Yield: $89 \%$<smiles>CC(=O)C(C(C)=O)C1=Cc2cc(-c3ccccc3)ccc2CN1</smiles>
(34mg), Physical appearance: white solid, M.p. $125-126{ }^{\circ} \mathrm{C}$; TLC $R_{f} 0.3$ (7:3, Petroleum ether: EtOAc); ${ }^{1} \mathbf{H}$ NMR $\left(500 \mathrm{MHz}^{\left.\mathrm{CDCl}_{3}\right)} \delta 7.58(\mathrm{dd}, J\right.$ $=8.3,1.3 \mathrm{~Hz}, 2 \mathrm{H}), 7.51-7.43(\mathrm{~m}, 4 \mathrm{H}), 7.41-7.35(\mathrm{~m}, 1 \mathrm{H}), 7.24(\mathrm{~d}, J=$ 
$7.7 \mathrm{~Hz}, 1 \mathrm{H}), 6.65(\mathrm{~s}, 1 \mathrm{H}), 5.01(\mathrm{~s}, 1 \mathrm{H}), 4.73(\mathrm{~s}, 2 \mathrm{H}), 3.85(\mathrm{~s}, 6 \mathrm{H}), 2.30(\mathrm{~s}, 3 \mathrm{H}) ;{ }^{13} \mathrm{C}$ NMR $(126$

$\left.\mathrm{MHz}, \mathrm{CDCl}_{3}\right) \delta 169.8,167.9,141.3,140.4,134.2,131.8,129.8,128.8,127.5,127.0,126.6$, 124.9, 124.5, 120.4, 55.2, 53.0, 49.8, 25.3, 22.6; IR (cm-1): 2950, 1758, 1726, 1655, 1409, 1397 , 1287, 1199; HRMS (ESI) $m / z:[\mathrm{M}+\mathrm{Na}]^{+}$calcd. for $\mathrm{C}_{22} \mathrm{H}_{21} \mathrm{NO}_{5} \mathrm{Na} 402.1312$; found 402.1334 .

Dimethyl 2-(2-acetyl-6-(4-methoxyphenyl)-1,2-dihydroisoquinolin-3-yl)malonate (3q):

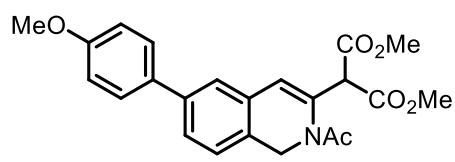

Yield: 78\% (32 mg); Physical appearance: pale-yellow solid; M.p. 53-54 ${ }^{\circ} \mathrm{C}$; TLC $R_{f} 0.3$ (7:3, Petroleum ether: EtOAc); ${ }^{1} \mathbf{H}$ NMR (500 $\left.\mathrm{MHz}, \mathrm{CDCl}_{3}\right) \delta 7.51(\mathrm{~d}, J=8.7 \mathrm{~Hz}, 2 \mathrm{H}), 7.43(\mathrm{dd}, J=7.7,1.8 \mathrm{~Hz}$ 1H), $7.41(\mathrm{~s}, 1 \mathrm{H}), 7.21(\mathrm{~d}, J=7.7 \mathrm{~Hz}, 1 \mathrm{H}), 7.00(\mathrm{~d}, J=8.7 \mathrm{~Hz}, 2 \mathrm{H}), 6.64(\mathrm{~s}, 1 \mathrm{H}), 5.00(\mathrm{~s}, 1 \mathrm{H})$, $4.71(\mathrm{~s}, 2 \mathrm{H}), 3.88(\mathrm{~s}, 3 \mathrm{H}), 3.84(\mathrm{~s}, 6 \mathrm{H}), 2.29(\mathrm{~s}, 3 \mathrm{H}) ;{ }^{13} \mathrm{C}$ NMR (176 MHz, CDCl 3 ) $\delta 169.8$, 167.9 , 159.3, 140.9, 134.1, 132.9, 131.7, 129.2, 128.0, 126.2, 124.8, 124.1, 120.4, 114.2, 55.3, 55.2, 53.0, 49.8, 22.6; IR (cm $\left.{ }^{-1}\right):$ 2940, 2144, 2128, 1757, 1708, 1689, 1452, 1324, 1272; HRMS (ESI) $m / z:[\mathrm{M}+\mathrm{Na}]^{+}$calcd. for $\mathrm{C}_{23} \mathrm{H}_{23} \mathrm{NO}_{6} \mathrm{Na} 432.1418$; found 432.1414 .

Dimethyl 2-(2-acetyl-6-bromo-1,2-dihydroisoquinolin-3-yl)malonate (3r): Yield: 58\% (22

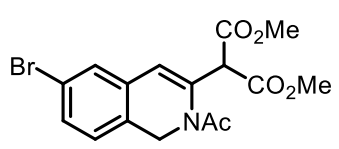
mg), Physical appearance: white solid, M.p. $133-134{ }^{\circ} \mathrm{C}$; TLC $R_{f} 0.3$ (7:3,

Petroleum ether: EtOAc); ${ }^{1} \mathbf{H}$ NMR $\left(500 \mathrm{MHz}^{\mathrm{CDCl}} 3\right) \delta 7.38-7.30(\mathrm{~m}$, 2H), $7.01(\mathrm{~d}, J=7.8 \mathrm{~Hz}, 1 \mathrm{H}), 6.48(\mathrm{~s}, 1 \mathrm{H}), 4.94(\mathrm{~s}, 1 \mathrm{H}), 4.60$ (s, 2H), 3.80 (s, 6H), 2.23 (s, 3H); ${ }^{13} \mathrm{C}$ NMR (126 MHz, $\left.\mathrm{CDCl}_{3}\right) \delta 169.8,167.7,135.3,133.2,130.6,129.5$, 128.4, 126.0, 121.7, 119.0, 55.1, 53.0, 49.5, 22.6; IR $\left(\mathrm{cm}^{-1}\right): 2950,1758,1726,1655,1409$, 1397, 1287, 1199; HRMS (ESI) $m / z:\left[\mathrm{M}+\mathrm{Na}^{+}\right.$calcd. for $\mathrm{C}_{16} \mathrm{H}_{16} \mathrm{BrNO}_{5} \mathrm{Na} 404.0104$ and 406.0084; found 404.0130 and 406.0110 .

\section{Dimethyl 2-(2-acetyl-7-phenyl-1,2-dihydroisoquinolin-3-yl)malonate (3s):}

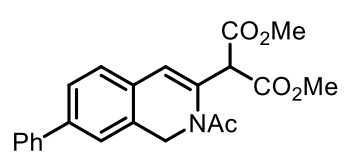

Yield: 78\% (30 mg); Physical appearance: white solid; M.p. 115-116 ${ }^{\circ} \mathrm{C}$; TLC $R_{f} 0.3$ (7:3, Petroleum ether: EtOAc); ${ }^{1} \mathbf{H}$ NMR $(500 \mathrm{MHz}$, $\left.\mathrm{CDCl}_{3}\right) \delta 7.60(\mathrm{dd}, J=8.3,1.3 \mathrm{~Hz}, 2 \mathrm{H}), 7.53(\mathrm{dd}, J=7.9,1.8 \mathrm{~Hz}, 1 \mathrm{H})$, $7.47(\mathrm{t}, J=7.7 \mathrm{~Hz}, 2 \mathrm{H}), 7.43-7.35(\mathrm{~m}, 2 \mathrm{H}), 7.29$ (d, J = $7.8 \mathrm{~Hz}, 2 \mathrm{H}), 6.62(\mathrm{~s}, 1 \mathrm{H}), 5.00(\mathrm{~s}, 1 \mathrm{H})$, 4.75 (s, 2H), 3.85 (s, 6H), 2.30 (s, 3H); $\left.{ }^{13} \mathbf{C ~ N M R ~ ( 1 7 6 ~ M H z , ~} \mathrm{CDCl}_{3}\right) \delta$ 169.8, 167.9, 140.9, $140.3,133.9,131.4,130.4,128.8,127.6,126.9,126.8,126.2$, 123.2, 120.0, 55.2, 53.0, 50.1, 22.6; IR $\left(\mathrm{cm}^{-1}\right): 2919,1702,1653,1618,1588,1471,1363,1197$; HRMS (ESI) $m / z: \mathrm{C}_{22} \mathrm{H}_{21} \mathrm{NO}_{5} \mathrm{Na}$ $[\mathrm{M}+\mathrm{Na}]^{+}$402.1312, found 402.1317 . 
Dimethyl 2-(2-acetyl-7-bromo-1,2-dihydroisoquinolin-3-yl)malonate (3t): Yield: 48\% (18

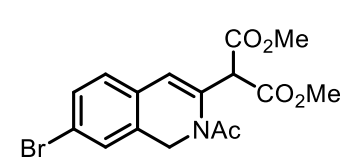

mg); Physical appearance: white solid; M.p. $112-113{ }^{\circ} \mathrm{C}$; TLC $R_{f} 0.3$ (7:3, Petroleum ether: EtOAc); ${ }^{1} \mathbf{H}$ NMR $\left(500 \mathrm{MHz} \mathrm{CDCl}_{3}\right) \delta 7.40(\mathrm{dd}, J=$ 8.1, $2.0 \mathrm{~Hz}, 1 \mathrm{H}), 7.30(\mathrm{~d}, J=1.9 \mathrm{~Hz}, 1 \mathrm{H}), 7.05(\mathrm{~d}, J=8.1 \mathrm{~Hz}, 1 \mathrm{H}), 6.52$

$(\mathrm{s}, 1 \mathrm{H}), 4.94(\mathrm{~s}, 1 \mathrm{H}), 4.62(\mathrm{~s}, 2 \mathrm{H}), 3.81(\mathrm{~s}, 6 \mathrm{H}), 2.24(\mathrm{~s}, 3 \mathrm{H}) ;{ }^{13} \mathbf{C} \mathbf{N M R}\left(176 \mathrm{MHz}, \mathrm{CDCl}_{3}\right) \delta$ $169.8,167.8$, 134.5, 132.6, 131.2, 130.3, 129.6, 127.6, 127.2, 121.4, 119.5, 55.1, 53.0, 49.4, 22.6; IR (cm $\left.{ }^{-1}\right): 2993,2953,1749,1732,1654,1480,1396,1279$; HRMS (ESI) m/z: [M + Na $]^{+}$ calcd. for $\mathrm{C}_{16} \mathrm{H}_{16} \mathrm{BrNO}_{5} \mathrm{Na} 404.0104$ and 406.0084; found 404.0112 and 406.0091 .

5-(1,4-dihydroisoquinolin-3(2H)-ylidene)-2,2-dimethyl-1,3-dioxane-4,6-dione (5a): Yield:

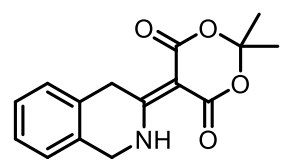

76\% (20 mg); Physical appearance: yellow solid, M.p. $155-157{ }^{\circ} \mathrm{C}$; TLC $R_{f}$ 0.3 (1:1, Petroleum ether: EtOAc); ${ }^{1} \mathbf{H}$ NMR (500 $\left.\mathrm{MHz}, \mathrm{CDCl}_{3}\right)$ : ${ }^{1} \mathrm{H} \mathrm{NMR}$ $\left(400 \mathrm{MHz}, \mathrm{CDCl}_{3}\right) \delta 11.89(\mathrm{~s}, 1 \mathrm{H}), 7.38-7.29(\mathrm{~m}, 3 \mathrm{H}), 7.23$ (d, J = 7.0 Hz,

$1 \mathrm{H}), 4.64(\mathrm{q}, J=2.6 \mathrm{~Hz}, 2 \mathrm{H}), 4.56(\mathrm{~d}, J=2.4 \mathrm{~Hz}, 2 \mathrm{H}), 1.72(\mathrm{~s}, 6 \mathrm{H}) ;{ }^{13} \mathbf{C ~ N M R}(101 \mathrm{MHz}$, $\left.\mathrm{CDCl}_{3}\right): \delta 172.4,167.5,163.1,129.8,129.8,128.3,128.2,127.3,125.1,102.8,83.5,45.1,33.4$, 26.4; IR (cm $\left.{ }^{-1}\right): 2994,1701,1648,1622,1594,1487,1391,1372,1292,1198 ;$ HRMS (ESI) $m / z:[\mathrm{M}+\mathrm{Na}]^{+}$calcd. for $\mathrm{C}_{15} \mathrm{H}_{15} \mathrm{NO}_{4} \mathrm{Na} 296.0898$; found 296.0893 .

2,2-dimethyl-5-(1-methyl-1,4-dihydroisoquinolin-3(2H)-ylidene)-1,3-dioxane-4,6-dione (5b):

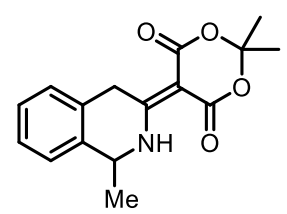

Yield: 68\% (19 mg); Physical appearance: pale-yellow solid, M.p. 157-158

${ }^{\circ} \mathrm{C}$; TLC R $\mathrm{R}_{f} 0.3$ (1:1, Petroleum ether: EtOAc); ${ }^{1} \mathbf{H}$ NMR $\left(400 \mathrm{MHz}, \mathrm{CDCl}_{3}\right) \delta$ $11.84(\mathrm{~s}, 1 \mathrm{H}), 7.32(\mathrm{~d}, J=3.1 \mathrm{~Hz}, 3 \mathrm{H}), 7.23-7.21(\mathrm{~m}, 1 \mathrm{H}), 4.86-4.68(\mathrm{~m}$, 1H), $4.67-4.40(\mathrm{~m}, 2 \mathrm{H}), 1.69(\mathrm{~s}, 6 \mathrm{H}), 1.66(\mathrm{~d}, J=6.8 \mathrm{~Hz}, 3 \mathrm{H}) ;{ }^{13} \mathbf{C}$ NMR $\left(101 \mathrm{MHz} \mathrm{CDCl}_{3}\right) \delta 171.7,167.4,163.2,134.9,129.3,128.2,128.0,127.5,124.2,102.7,83.2$, 50.9, 33.2, 26.6, 26.2, 22.1; IR (cm $\left.{ }^{-1}\right):$ 2922, 1704, 1647, 1610, 1522, 1482, 1390, 1292, 1152; HRMS (ESI) $m / z:[\mathrm{M}+\mathrm{K}]^{+}$calcd. for $\mathrm{C}_{16} \mathrm{H}_{17} \mathrm{NO}_{4} \mathrm{~K} 326.0789$; found 326.0800 .

5-(5-(4-methoxyphenyl)-1,4-dihydroisoquinolin-3(2H)-ylidene)-2,2-dimethyl-1,3-dioxane-

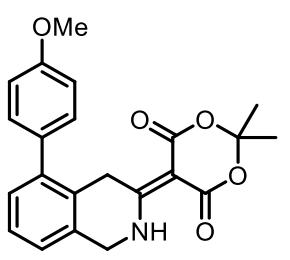

4,6-dione (5c): Yield: 60\% (22 mg); Physical appearance: pale-yellow solid, M.p. $179-180{ }^{\circ} \mathrm{C}$; TLC $R_{f} 0.3$ (1:1, Petroleum ether: EtOAc); ${ }^{1} \mathbf{H}$ NMR (500 $\left.\mathrm{MHz} \mathrm{CDCl}_{3}\right) \delta 11.85(\mathrm{~s}, 1 \mathrm{H}), 7.38-7.31(\mathrm{~m}, 2 \mathrm{H}), 7.30(\mathrm{~d}, J=8.6 \mathrm{~Hz}, 2 \mathrm{H})$, $7.22(\mathrm{dd}, J=7.3,1.6 \mathrm{~Hz}, 1 \mathrm{H}), 7.02(\mathrm{~d}, J=8.6 \mathrm{~Hz}, 2 \mathrm{H}), 4.66(\mathrm{q}, J=2.4 \mathrm{~Hz}$, 2H), $4.55(\mathrm{~d}, J=2.0 \mathrm{~Hz}, 2 \mathrm{H}), 3.87(\mathrm{~s}, 3 \mathrm{H}), 1.66(\mathrm{~s}, 6 \mathrm{H}) ;{ }^{13} \mathbf{C} \mathbf{N M R}\left(126 \mathrm{MHz}, \mathrm{CDCl}_{3}\right) \delta 172.8$, 
$167.4,162.9,159.2,141.4,131.5,130.9,130.3,129.9,127.7,127.1,124.0,114.0,102.6,83.4$, 55.2, 45.5, 31.4, 26.4; IR ( $\left.\mathrm{cm}^{-1}\right):$ 2997, 1704, 1654, 1593, 1472, 1440, 1362, 1267, 1197; HRMS (ESI) $m / z:[\mathrm{M}+\mathrm{Na}]^{+}$calcd. for $\mathrm{C}_{22} \mathrm{H}_{21} \mathrm{NO}_{5} \mathrm{Na}$ 402.1312; found 402.1337 .

2,2-dimethyl-5-(6-phenyl-1,4-dihydroisoquinolin-3(2H)-ylidene)-1,3-dioxane-4,6-dione (5d):

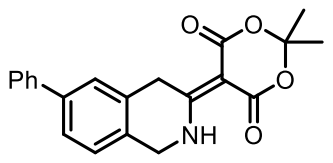

Yield: 82\% (28 mg); Physical appearance: white solid, M.p. $145-146{ }^{\circ} \mathrm{C}$;

TLC $\mathrm{R}_{f} 0.3$ (1:1, Petroleum ether: EtOAc); ${ }^{\mathbf{1}} \mathbf{H} \mathbf{N M R}\left(500 \mathrm{MHz}, \mathrm{CDCl}_{3}\right) \delta$ $11.91(\mathrm{~s}, 1 \mathrm{H}), 7.58$ (d, $J=7.2 \mathrm{~Hz}, 3 \mathrm{H}), 7.53(\mathrm{dd}, J=7.9,1.9 \mathrm{~Hz}, 1 \mathrm{H}), 7.46$ (t, $J=7.7 \mathrm{~Hz}, 2 \mathrm{H}), 7.40-7.35(\mathrm{~m}, 1 \mathrm{H}), 7.29(\mathrm{~d}, J=7.9 \mathrm{~Hz}, 1 \mathrm{H}), 4.68-4.66(\mathrm{~m}, 2 \mathrm{H}), 4.62(\mathrm{~d}, J$ $=2.4 \mathrm{~Hz}, 2 \mathrm{H}), 1.71(\mathrm{~s}, 6 \mathrm{H}) ;{ }^{13} \mathbf{C} \mathbf{N M R}\left(126 \mathrm{MHz}, \mathrm{CDCl}_{3}\right) \delta 172.4,167.5,163.2,141.5,140.1$, $130.3,128.9,128.7,127.7,127.0,126.9,126.8,126.2,125.6,102.8,83.6,44.9$, 33.5, 26.5; IR $\left(\mathrm{cm}^{-1}\right):$ 2994, 1701, 1648, 1622, 1594, 1487, 1391, 1372, 1292, 1198; HRMS (ESI) m/z: [M + $\mathrm{H}]^{+}$calcd. for $\mathrm{C}_{21} \mathrm{H}_{20} \mathrm{NO}_{4} 350.1387$; found 350.1414 .

5-(7-bromo-1,4-dihydroisoquinolin-3(2H)-ylidene)-2,2-dimethyl-1,3-dioxane-4,6-dione (5e):

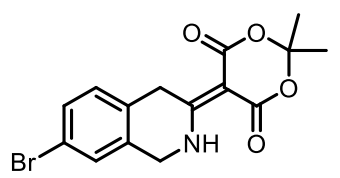

Yield:77\% (27 mg); Physical appearance: yellow solid; M.p. 170-172 ${ }^{\circ} \mathrm{C}$;

TLC $R_{f} 0.3$ (7:3, Petroleum ether: EtOAc); ${ }^{1} \mathbf{H}$ NMR $\left(400 \mathrm{MHz}, \mathrm{CDCl}_{3}\right) \delta$ 11.89 (s, 1H), 7.48 (dd, $J=8.2,2.0 \mathrm{~Hz}, 1 \mathrm{H}), 7.39$ (s, 1H), 7.23 (d, $J=8.2$ $\mathrm{Hz}, 1 \mathrm{H}), 4.62(\mathrm{q}, J=2.6 \mathrm{~Hz}, 2 \mathrm{H}), 4.52(\mathrm{~d}, J=2.4 \mathrm{~Hz}, 2 \mathrm{H}), 1.72(\mathrm{~s}, 6 \mathrm{H}) ;{ }^{13} \mathbf{C} \mathbf{N M R}(126 \mathrm{MHz}$, $\left.\mathrm{CDCl}_{3}\right) \delta 171.8,167.4,163.0,131.9,131.4,129.8,128.8,128.1,121.0,102.9,83.6,44.5,32.8$, 26.4; IR (cm-1): 2976, 1742, 1645, 1601, 1563, 1491, 1327, 1206; HRMS (ESI) m/z: [M + Na] ${ }^{+}$ calcd. for $\mathrm{C}_{15} \mathrm{H}_{14} \mathrm{BrNO}_{4} \mathrm{Na} 373.9998$ and 375.9979; found 373.9996 and 375.9979.

Methyl 2-(2-acetyl-1,2-dihydroisoquinolin-3-yl)-3-oxo-3-phenylpropanoate (7a): Yield 75\%

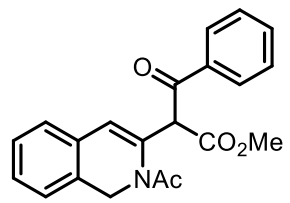

(25 mg), Physical appearance: pale-yellow solid, M.p. 89-90 ${ }^{\circ} \mathrm{C}$, TLC $R_{f} 0.40$ (7:3, Petroleum ether: EtOAc); ${ }^{1} \mathbf{H}$ NMR $\left(500 \mathrm{MHz}, \mathrm{CDCl}_{3}\right) \delta 8.16(\mathrm{~d}, J=$ $7.7 \mathrm{~Hz}, 2 \mathrm{H}), 7.58$ (t, $J=7.4 \mathrm{~Hz}, 1 \mathrm{H}), 7.48(\mathrm{t}, J=7.7 \mathrm{~Hz}, 2 \mathrm{H}), 7.24-7.17$ (m, 2H), $7.14-7.04(\mathrm{~m}, 2 \mathrm{H}), 6.34(\mathrm{~s}, 1 \mathrm{H}), 6.14(\mathrm{~s}, 1 \mathrm{H}), 4.72$ (d, $J=15.0 \mathrm{~Hz}, 1 \mathrm{H}), 4.57$ (d, J= 15.0 $\mathrm{Hz}, 1 \mathrm{H}), 3.81$ (s, 3H), 2.30 (s, 3H); ${ }^{13} \mathrm{C}$ NMR (126 MHz, $\left.\mathrm{CDCl}_{3}\right) \delta$ 193.1, 170.2, 169.0, 135.4, 134.5, 133.7, 131.3, 131.1, 129.1, 128.8, 128.8, 128.1, 128.0, 125.8, 124.4, 121.8, 57.7, 52.7, 50.1, 31.5, 22.8, 22.6, 14.1; IR (KBr, cm $\left.{ }^{-1}\right):$ 2986, 1783, 1647, 1338, 1250, 1051; HRMS (ESI) $m / z:[\mathrm{M}+\mathrm{Na}]^{+}$calcd. for $\mathrm{C}_{21} \mathrm{H}_{19} \mathrm{NO}_{4} \mathrm{Na} 372.1206$; found 372.1215 . 
Ethyl 2-(2-acetyl-1,2-dihydroisoquinolin-3-yl)-3-oxo-3-phenylpropanoate (7b): Yield: 77\%

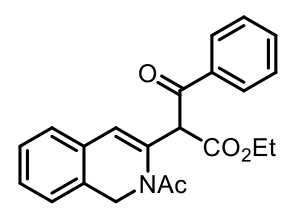

(28 mg), Physical appearance: colorless solid, M.p. 89-91 ${ }^{\circ} \mathrm{C}$, TLC $R_{f} 0.40$ (7:3, Petroleum ether: EtOAc); ${ }^{1} \mathbf{H}$ NMR $\left(500 \mathrm{MHz}, \mathrm{CDCl}_{3}\right) \delta 8.17(\mathrm{~d}, \mathrm{~J}=7.7$ $\mathrm{Hz}, 2 \mathrm{H}), 7.62-7.54(\mathrm{~m}, 1 \mathrm{H}), 7.48(\mathrm{dd}, \mathrm{J}=8.4,7.1 \mathrm{~Hz}, 2 \mathrm{H}), 7.24-7.18(\mathrm{~m}$, 2H), $7.14-7.05(\mathrm{~m}, 2 \mathrm{H}), 6.34(\mathrm{~s}, 1 \mathrm{H}), 6.19-6.07(\mathrm{~m}, 1 \mathrm{H}), 4.71(\mathrm{~d}, \mathrm{~J}=15.0 \mathrm{~Hz}, 1 \mathrm{H}), 4.58$ (d, J $=15.1 \mathrm{~Hz}, 1 \mathrm{H}), 4.34-4.21(\mathrm{~m}, 2 \mathrm{H}), 2.30(\mathrm{~s}, 3 \mathrm{H}), 1.28(\mathrm{t}, \mathrm{J}=7.1 \mathrm{~Hz}, 3 \mathrm{H}) ;{ }^{13} \mathbf{C ~ N M R}(176 \mathrm{MHz}$, $\left.\mathrm{CDCl}_{3}\right) \delta 193.2,170.2,168.5,135.5,134.6,133.6,131.4,131.1,129.1,128.8,128.1,127.9$, 125.8, 124.3, 121.7, 61.8, 57.8, 50.2, 22.9, 14.0; IR $\left(\mathrm{KBr}, \mathrm{cm}^{-1}\right): 2979,1781,1665,1495,1258$, 1175; HRMS (ESI) $m / z:[\mathrm{M}+\mathrm{H}]^{+}$calcd. for $\mathrm{C}_{22} \mathrm{H}_{22} \mathrm{NO}_{4} 364.1543$; found 364.1527.

Benzyl 2-(2-acetyl-1,2-dihydroisoquinolin-3-yl)-3-oxo-3-phenylpropanoate (mixture of

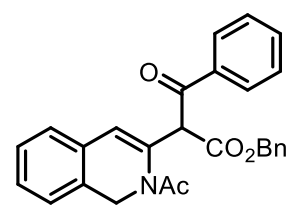
rotamers) (7c): Yield: $73 \%$ (30 mg), Physical appearance: yellow gel, TLC $R_{f} 0.40$ (7:3, Petroleum ether: EtOAc); ${ }^{1} \mathbf{H}$ NMR $(500 \mathrm{MHz}, \mathrm{CDCl} 3) \delta 8.17$ $(\mathrm{dd}, \mathrm{J}=13.9,7.7 \mathrm{~Hz}, 2 \mathrm{H}), 7.60-7.53(\mathrm{~m}, 1 \mathrm{H}), 7.51-7.44(\mathrm{~m}, 2 \mathrm{H}), 7.41-$ $7.38(\mathrm{~m}, 1 \mathrm{H}), 7.37-7.30(\mathrm{~m}, 5 \mathrm{H}), 7.24-7.18(\mathrm{~m}, 2 \mathrm{H}), 7.13-7.08(\mathrm{~m}, 1 \mathrm{H})$, $7.07-7.02(\mathrm{~m}, 1 \mathrm{H}), 6.35(\mathrm{~d}, \mathrm{~J}=9.4 \mathrm{~Hz}, 1 \mathrm{H}), 6.22(\mathrm{~s}, 1 \mathrm{H}), 5.31(\mathrm{~d}, \mathrm{~J}=12.3 \mathrm{~Hz}, 1 \mathrm{H}), 5.24(\mathrm{~d}, \mathrm{~J}=$ $12.4 \mathrm{~Hz}, 1 \mathrm{H}), 4.70(\mathrm{dd}, \mathrm{J}=23.2,15.2 \mathrm{~Hz}, 1 \mathrm{H}), 4.55(\mathrm{~d}, \mathrm{~J}=15.0 \mathrm{~Hz}, 1 \mathrm{H}), 2.32-2.24(\mathrm{~m}, 3 \mathrm{H})$; ${ }^{13} \mathrm{C}$ NMR (176 MHz, $\left.\mathrm{CDCl}_{3}\right) \delta 192.9,170.2,135.5,133.7,131.3,131.1,129.1,128.8,128.6$, $128.5,128.5,128.2$, 128.2, 128.1, 128.0, 125.8, 124.4, 121.8, 67.4, 57.8, 50.1, 22.9, 22.8, 20.1; IR $\left(\mathbf{c m}^{-1}\right): 2975,1768,1653,1418,1286,1155 ;$ HRMS (ESI) $m / z:[\mathrm{M}+\mathrm{Na}]^{+}$calcd. for $\mathrm{C}_{27} \mathrm{H}_{23} \mathrm{NO}_{4} \mathrm{Na} 448.1519$; found 448.1546 .

\section{Methyl-(2S)-2-(2-acetyl-1-methyl-1,2-dihydroisoquinolin-3-yl)-3-oxo-3-phenylpropanoate}

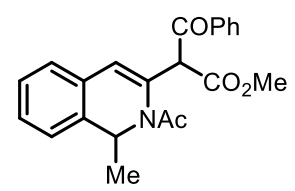

(7d): Yield 68\% (24 mg), Physical appearance: pale-yellow gel, TLC $R_{f} 0.40$ (7:3, Petroleum ether: EtOAc) (mixture of rotamers and inseparable diastereomers); ${ }^{1} \mathbf{H}$ NMR (400 MHz, $\left.\mathrm{CDCl}_{3}\right) \delta 8.23(\mathrm{~d}, J=7.7 \mathrm{~Hz}, 1 \mathrm{H}), 7.96$ - $7.88(\mathrm{~m}, 2 \mathrm{H}), 7.57-7.53(\mathrm{~m}, 2 \mathrm{H}), 7.48-7.42(\mathrm{~m}, 4 \mathrm{H}), 7.21-7.16(\mathrm{~m}$,

3H), $7.15-7.11(\mathrm{~m}, 1 \mathrm{H}), 7.08-7.04(\mathrm{~m}, 1 \mathrm{H}), 7.02-7.0(\mathrm{~m}, 1 \mathrm{H}), 6.4(\mathrm{~d}, J=9.8 \mathrm{~Hz}, 2 \mathrm{H}), 5.67$ (s, 1H), $5.03(\mathrm{~d}, J=10.0 \mathrm{~Hz}, 1 \mathrm{H}), 3.84(\mathrm{~s}, 3 \mathrm{H}), 3.74$ (s, 2H), 2.33 (s, 3H), 2.31 (s, 2H), 1.43 (d, $J$ $=6.8 \mathrm{~Hz}, 2 \mathrm{H}), 1.06(\mathrm{~d}, J=6.8 \mathrm{~Hz}, 3 \mathrm{H}) ;{ }^{13} \mathbf{C ~ N M R}\left(126 \mathrm{MHz}, \mathrm{CDCl}_{3}\right): \delta 192.6,170.1,168.5$, 135.7, 133.7, 129.3, 129.2, 128.8, 128.0, 128.0, 127.8, 127.8, 126.1, 126.1, 124.5, 77.3, 58.2, 52.7, 27.1, 23.06, 21.3, 20.7; IR (KBr, cm $\left.{ }^{-1}\right): 2979,1751,1667,1490,1258,1175$; HRMS (ESI) $m / z:[\mathrm{M}+\mathrm{Na}]^{+}$calcd. for $\mathrm{C}_{22} \mathrm{H}_{21} \mathrm{NO}_{4} \mathrm{Na} 386.1363$; found 386.1370. 


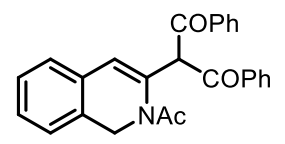

rotamers) (9a): Yield: 76\% (29 mg), Physical appearance: pale-yellow solid,

M.p. $148-150{ }^{\circ} \mathrm{C}$ TLC $R_{f} 0.30$ (7:3, Petroleum ether: EtOAc); ${ }^{1} \mathbf{H}$ NMR (500 $\mathrm{MHz}_{\mathrm{CDCl}} \mathrm{CD}_{3} \delta .13(\mathrm{~d}, \mathrm{~J}=7.7 \mathrm{~Hz}, 4 \mathrm{H}), 7.57(\mathrm{t}, \mathrm{J}=7.4 \mathrm{~Hz}, 2 \mathrm{H}), 7.47$ (t, J = 7.8 Hz, 4H), 7.38 $(\mathrm{s}, 1 \mathrm{H}), 7.21(\mathrm{dd}, \mathrm{J}=5.6,3.2 \mathrm{~Hz}, 2 \mathrm{H}), 7.18-7.12(\mathrm{~m}, 1 \mathrm{H}), 7.08-7.01(\mathrm{~m}, 1 \mathrm{H}), 6.24(\mathrm{~s}, 1 \mathrm{H})$, $4.74(\mathrm{~s}, 2 \mathrm{H}), 2.28$ (s, 3H); ${ }^{13} \mathrm{C}$ NMR (176 MHz, $\left.\mathrm{CDCl}_{3}\right) \delta$ 194.3, 170.4, 135.9, 135.3, 133.6, 131.5, 131.5, 128.9, 128.9, 128.0, 127.9, 125.8, 124.4, 122.5, 59.7, 50.2, 23.0; IR $\left(\mathrm{cm}^{-1}\right): 2918$, 2851, 1741, 1682, 1660, 1579, 1486, 1391, 1291, 1200; HRMS (ESI) $m / z:[\mathrm{M}+\mathrm{Na}]^{+}$calcd. for $\mathrm{C}_{26} \mathrm{H}_{21} \mathrm{NO}_{3} \mathrm{Na} 418.1422$; found 418.1414 .

2-(2-acetyl-1-methyl-1,2-dihydroisoquinolin-3-yl)-1,3-diphenylpropane-1,3-dione

(9b): Yield: 78\% (32 mg), Physical appearance: pale-yellow solid, M.p. 170-171

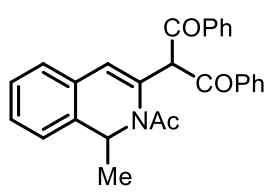

${ }^{\circ} \mathrm{C}$; TLC $R_{f} 0.3$ (7:3, Petroleum ether: EtOAc); ${ }^{1} \mathbf{H}$ NMR $\left(500 \mathrm{MHz}, \mathrm{CDCl}_{3}\right) \delta$ $8.16(\mathrm{~d}, J=7.7 \mathrm{~Hz}, 2 \mathrm{H}), 8.01-7.96(\mathrm{~m}, 2 \mathrm{H}), 7.59-7.51(\mathrm{~m}, 2 \mathrm{H}), 7.49-7.42$ $(\mathrm{m}, 4 \mathrm{H}), 7.25(\mathrm{~s}, 1 \mathrm{H}), 7.22-7.16(\mathrm{~m}, 2 \mathrm{H}), 7.11-7.07(\mathrm{~m}, 1 \mathrm{H}), 7.07-7.02$

$(\mathrm{m}, 1 \mathrm{H}), 6.25(\mathrm{~s}, 1 \mathrm{H}), 5.05(\mathrm{~s}, 1 \mathrm{H}), 2.32(\mathrm{~s}, 3 \mathrm{H}), 1.31(\mathrm{~d}, \mathrm{~J}=7.0 \mathrm{~Hz}, 3 \mathrm{H}) ;{ }^{13} \mathbf{C ~ N M R}(126 \mathrm{MHz}$, $\left.\mathrm{CDCl}_{3}\right) \delta 193.2,193.1,170.3,136.3,136.2,135.3,133.6,133.2,129.9,129.3,129.0,128.9$, 128.8, 128.8, 128.6, 127.9, 127.8, 127.8, 126.0, 124.5, 120.8, 58.8, 55.3, 23.2, 21.3; IR (cm $\left.{ }^{-1}\right)$ : 2974, 1687, 1659, 1579, 1447, 1402, 1312, 1279, 1200; HRMS (ESI) $m / z:[\mathrm{M}+\mathrm{Na}]^{+}$calcd. for $\mathrm{C}_{27} \mathrm{H}_{23} \mathrm{NO}_{3} \mathrm{Na}$ 432.1570; found 432.1541.

\section{2-(2-acetyl-1,2-dihydroisoquinolin-3-yl)-1,3-bis(4-methoxyphenyl)propane-1,3-dione}

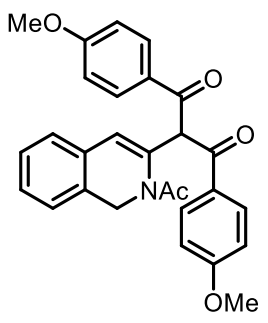

(mixture of rotamers) (9c): Yield: 68\% (30 mg), Physical appearance: yellow gel, TLC $R_{f} 0.30$ (7:3, Petroleum ether: EtOAc); ${ }^{1} \mathbf{H}$ NMR $\left(500 \mathrm{MHz}, \mathrm{CDCl}_{3}\right)$ $\delta 8.13(\mathrm{~m}, 4 \mathrm{H}), 7.32(\mathrm{~s}, 1 \mathrm{H}), 7.22-7.18(\mathrm{~m}, 2 \mathrm{H}), 7.16-7.13(\mathrm{~m}, 1 \mathrm{H}), 7.03$ $-7.01(\mathrm{~m}, 1 \mathrm{H}), 6.93(\mathrm{~m}, 4 \mathrm{H}), 6.24(\mathrm{~s}, 1 \mathrm{H}), 4.75(\mathrm{~s}, 2 \mathrm{H}), 3.85(\mathrm{~s}, 6 \mathrm{H}), 2.26(\mathrm{~s}$, $3 \mathrm{H}) ;{ }^{13} \mathrm{C}$ NMR $\left(126 \mathrm{MHz}, \mathrm{CDCl}_{3}\right) \delta 193.2,170.4,163.9,131.7,131.3,129.0$, 127.9, 127.7, 125.76, 124.3, 122.3, 114.0, 99.9, 59.3, 55.4, 31.5, 23.0; IR ( $\left.\mathrm{cm}^{-1}\right):$ 2922, 2850, 1660, 1598, 1574, 1510, 1419, 1396, 1258, 1168; HRMS (ESI) $m / z:[\mathrm{M}+\mathrm{H}]^{+}$calcd. for $\mathrm{C}_{28} \mathrm{H}_{26} \mathrm{NO}_{5} 456.1805$; found 456.1807 .

2-(2-acetyl-5-phenyl-1,2-dihydroisoquinolin-3-yl)-1,3-diphenylpropane-1,3-dione (mixture<smiles>O=C(c1ccccc1)C(C(=O)c1ccccc1)c1cc2ccccc2c2ccccc12</smiles>
of rotamers) (9d): Yield: 65\% (31 mg), Physical appearance: yellow gel, TLC $R_{f} 0.3$ (7:3, Petroleum ether: EtOAc); ${ }^{1} \mathbf{H} \mathbf{N M R}\left(500 \mathrm{MHz}, \mathrm{CDCl}_{3}\right) \delta$ 
$8.18(\mathrm{~d}, J=7.8 \mathrm{~Hz}, 4 \mathrm{H}), 7.64(\mathrm{~d}, J=6.6 \mathrm{~Hz}, 2 \mathrm{H}), 7.53(\mathrm{t}, J=7.6 \mathrm{~Hz}, 4 \mathrm{H}), 7.34-7.24(\mathrm{~m}, 3 \mathrm{H})$, $7.18(\mathrm{dd}, J=13.2,7.2 \mathrm{~Hz}, 2 \mathrm{H}), 6.98(\mathrm{t}, J=7.5 \mathrm{~Hz}, 2 \mathrm{H}), 6.92(\mathrm{~d}, J=7.6 \mathrm{~Hz}, 2 \mathrm{H}), 6.07$ (s, 1H), $4.81(\mathrm{~s}, 2 \mathrm{H}), 2.33$ (s, 3H); ${ }^{13} \mathrm{C}$ NMR (126 MHz, $\left.\mathrm{CDCl}_{3}\right) \delta$ 195.0, 170.6, 138.7, 138.7, 135.9, 133.6, 133.3, 129.2, 129.1, 129.0, 128.8, 128.0, 127.8, 127.0, 123.4, 122.3, 99.9, 59.8, 50.7, 31.6, 23.0; IR $\left(\mathrm{cm}^{-1}\right): 2921,2851,1743,1660,1580,1461,1393,1257,1103$; HRMS (ESI) $m / z:[\mathrm{M}+$ $\mathrm{H}]^{+}$calcd. for $\mathrm{C}_{32} \mathrm{H}_{26} \mathrm{NO}_{3} 472.1907$; found 472.1894 .

2-(2-acetyl-1-phenyl-1,2-dihydroisoquinolin-3-yl)-1,3-diphenylpropane-1,3-dione

$(9 \mathrm{e})$ :

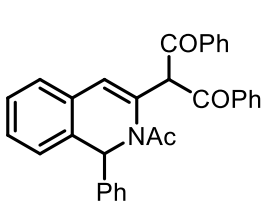

Yield: 54\% (26 mg), Physical appearance: pale-yellow gel, TLC $R_{f} 0.3$ (7:3,

Petroleum ether: EtOAc); ${ }^{1} \mathbf{H}$ NMR $\left(500 \mathrm{MHz}^{\left.\mathrm{CDCl}_{3}\right)} \delta 7.88(\mathrm{~d}, J=7.7 \mathrm{~Hz}\right.$, 2H), $7.51(\mathrm{~d}, J=7.8 \mathrm{~Hz}, 2 \mathrm{H}), 7.49-7.44(\mathrm{~m}, 1 \mathrm{H}), 7.44-7.39(\mathrm{~m}, 1 \mathrm{H}), 7.36(\mathrm{t}$, $J=7.6 \mathrm{~Hz}, 2 \mathrm{H}), 7.33(\mathrm{~d}, J=4.3 \mathrm{~Hz}, 2 \mathrm{H}), 7.25-7.21(\mathrm{~m}, 1 \mathrm{H}), 7.18-7.05(\mathrm{~m}, 6 \mathrm{H}), 6.96(\mathrm{~d}, J=$ $7.5 \mathrm{~Hz}, 2 \mathrm{H}), 6.59(\mathrm{~s}, 1 \mathrm{H}), 6.49(\mathrm{~s}, 1 \mathrm{H}), 6.19(\mathrm{~s}, 1 \mathrm{H}), 2.53(\mathrm{~s}, 3 \mathrm{H}) ;{ }^{13} \mathbf{C ~ N M R}\left(176 \mathrm{MHz}, \mathrm{CDCl}_{3}\right) \delta$ 191.2 , 170.5, 139.4, 133.0, 130.9, 128.7, 128.6, 128.5, 128.4, 128.2, 127.4, 126.3, 59.7, 31.6, 23.6, 22.6, 14.1; IR $\left(\mathrm{cm}^{-1}\right): 2918,1708,1666,1449,1268$; HRMS (ESI) $m / z:[\mathrm{M}+\mathrm{H}]^{+} \mathrm{calcd}$. for $\mathrm{C}_{32} \mathrm{H}_{26} \mathrm{NO}_{3} 472.1907$; found 472.1892.

2-(2-acetyl-1,2-dihydroisoquinolin-3-yl)-5,5-dimethylcyclohexane-1,3-dione (9f): Yield: $48 \%$

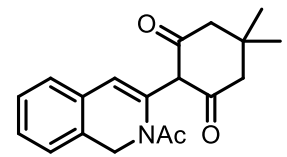

(15 mg), Physical appearance: yellow gel, TLC $R_{f} 0.2$ (7:3, Petroleum ether: EtOAc); ${ }^{1} \mathbf{H}$ NMR (enol form)(500 MHz, $\left.\mathrm{CDCl}_{3}\right) \delta 14.01(\mathrm{~s}, 1 \mathrm{H}), 7.45$ (d, $J=$ $6.8 \mathrm{~Hz}, 1 \mathrm{H}), 7.40-7.36(\mathrm{~m}, 1 \mathrm{H}), 7.35-7.32(\mathrm{~m}, 1 \mathrm{H}), 7.22(\mathrm{~d}, J=7.4 \mathrm{~Hz}$, 1H), $6.24(\mathrm{~s}, 1 \mathrm{H}), 4.68(\mathrm{~d}, J=16.8 \mathrm{~Hz}, 1 \mathrm{H}), 4.52(\mathrm{dd}, J=16.8,4.8 \mathrm{~Hz}, 1 \mathrm{H}), 2.47$ (s, 3H), $2.42(\mathrm{~s}$, 2H), $2.40-2.28(\mathrm{~m}, 2 \mathrm{H}), 1.04(\mathrm{~d}, J=6.1 \mathrm{~Hz}, 6 \mathrm{H}) ;{ }^{13} \mathbf{C}$ NMR $\left(126 \mathrm{MHz}, \mathrm{CDCl}_{3}\right) \delta$ 201.1, 199.2, 196.7, 169.8, 131.2, 128.8, 128.5, 128.4, 128.1, 126.0, 106.3, 58.0, 52.8, 51.9, 44.6, 30.3, 28.4, 28.3, 28.2; IR $\left(\mathrm{cm}^{-1}\right): 2955,2868,1768,1713,1634,1577,1465,1320,1289,1143$; HRMS (ESI) $m / z:[\mathrm{M}+\mathrm{H}]^{+}$calcd. for $\mathrm{C}_{19} \mathrm{H}_{22} \mathrm{NO}_{3}$ 312.1594; found 312.1590.

\section{v) Aromatization and Synthetic Applications:}

Scheme S13: Removal of activating tether and aromatization of C(3)-H alkylated product: 


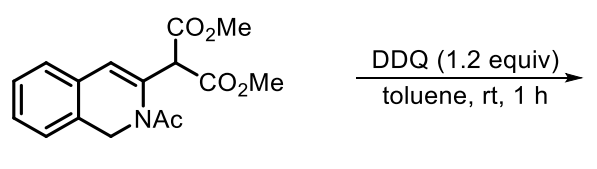

3b

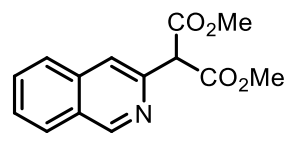

10

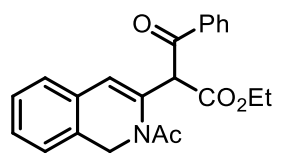

$7 b$ $\underset{\text { 1, 4-dioxane, } \mathrm{rt}, 1 \mathrm{~h}}{\stackrel{\mathrm{D}}{\mathrm{N}}}$

1, 4-dioxane, $\mathrm{rt}, 1 \mathrm{~h}$

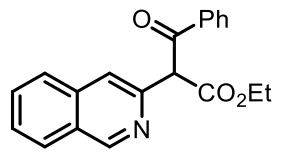

11

In a reaction tube charged with C(3)-alkylated product $\mathbf{3 b} / 7 \mathbf{b}(0.1 \mathrm{mmol})$ in toluene (for $\mathbf{3 b}) / 1,4$ dioxane (for 7b) (1 mL) at room temperature, DDQ (1.2 equiv) was added. The resulting solution was stirred vigorously for an hour at room temperature. The reaction was monitored by TLC. The mixture was then quenched with water $(10 \mathrm{~mL})$, extracted with DCM and dried over $\mathrm{Na}_{2} \mathrm{SO}_{4}$. After concentration of the organic extract under reduced pressure, the crude product was purified by a silica gel flash column chromatography (eluted with 7:3, Petroleum ether: EtOAc).

\section{Dimethyl-2-(isoquinolin-3-yl)malonate (10):}

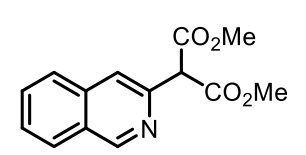

Yield: 80\% (28 mg); Physical appearance: pale-yellow semi-solid; TLC $R_{f}$ 0.50 (1:3, Petroleum ether: EtOAc); ${ }^{1} \mathbf{H}$ NMR $\left(500 \mathrm{MHz}, \mathrm{CDCl}_{3}\right) \delta 9.26(\mathrm{~s}$, 1H), $8.01(\mathrm{~d}, J=8.2 \mathrm{~Hz}, 1 \mathrm{H}), 7.87(\mathrm{~d}, J=7.2 \mathrm{~Hz}, 2 \mathrm{H}), 7.75-7.72(\mathrm{~m}, 1 \mathrm{H})$, $7.67-7.63(\mathrm{~m}, 1 \mathrm{H}), 5.16(\mathrm{~s}, 1 \mathrm{H}), 3.84(\mathrm{~s}, 6 \mathrm{H}) ;{ }^{13} \mathrm{C} \mathrm{NMR}\left(176 \mathrm{MHz}, \mathrm{CDCl}_{3}\right) \delta 168.2,152.4$, 146.1, 136.2, 130.7, 127.9, 127.7, 127.5, 126.8, 120.3, 59.8, 53.0; IR $\left(\mathrm{cm}^{-1}\right): 2986,2907,1716$, 1625, 1497, 1355, 1210; HRMS (ESI) $m / z:[\mathrm{M}+\mathrm{Na}]^{+}$calcd. for $\mathrm{C}_{14} \mathrm{H}_{13} \mathrm{NO}_{4} \mathrm{Na} 282.0737$; found 282.0718 .

Ethyl 2-(isoquinolin-3-yl)-3-oxo-3-phenylpropanoate (11): Yield: 41\% (16 mg), Physical appearance: colorless gel, TLC $R_{f} 0.50\left(7: 3\right.$, Petroleum ether: EtOAc); ${ }^{1} \mathbf{H}$
$\mathrm{NMR}\left(400 \mathrm{MHz}, \mathrm{CDCl}_{3}\right) \delta 9.22(\mathrm{~d}, \mathrm{~J}=1.0 \mathrm{~Hz}, 1 \mathrm{H}), 8.14-8.06(\mathrm{~m}, 2 \mathrm{H}), 7.98$ - $7.92(\mathrm{~m}, 1 \mathrm{H}), 7.85(\mathrm{~d}, \mathrm{~J}=0.9 \mathrm{~Hz}, 1 \mathrm{H}), 7.84-7.79(\mathrm{~m}, 1 \mathrm{H}), 7.70-7.65(\mathrm{~m}$, 1H), $7.64-7.56(\mathrm{~m}, 1 \mathrm{H}), 7.12-7.04(\mathrm{~m}, 2 \mathrm{H}), 6.05(\mathrm{~s}, 1 \mathrm{H}), 4.24-4.23(\mathrm{~m}, 2 \mathrm{H}), 1.28(\mathrm{t}, \mathrm{J}=7.1$ $\mathrm{Hz}, 3 \mathrm{H}) ;{ }^{13} \mathbf{C} \mathbf{N M R}\left(176 \mathrm{MHz}, \mathrm{CDCl}_{3}\right): \delta$ 187.8, 165.6, 152.7, 144.2, 135.1, 128.7, 127.8, 123.2, 62.2, 29.7, 14.1; IR (KBr, cm $\left.{ }^{-1}\right): 2979,2871,1734,1656,1404,1255,1027$; HRMS (ESI) m/z: $[\mathrm{M}+\mathrm{H}]^{+}$calcd. for $\mathrm{C}_{20} \mathrm{H}_{17} \mathrm{NO}_{3} 320.1281$; found 320.1304 . 
Scheme S14: Selective decarbonylation:

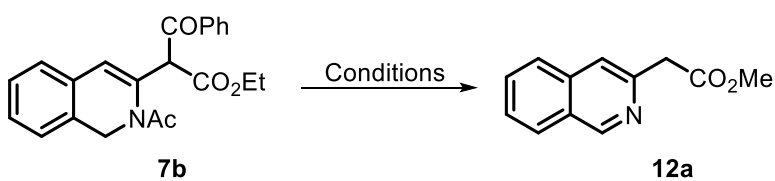

Table S3: Optimization of reaction conditions for decarbonylation:

\begin{tabular}{|c|c|c|}
\hline Entry & Conditions & Yield of 12a (\%) $^{\boldsymbol{a}}$ \\
\hline 1 & $p$-TsOH $(1.1$ equiv)/ethylene glycol/benzene/reflux/6 h & $\mathrm{NR}$ \\
\hline 2 & $p$-TsOH $\left(1.1\right.$ equiv)/ethylene glycol/ toluene $/ 110^{\circ} \mathrm{C} / 6 \mathrm{~h}$ & $\mathrm{NR}$ \\
\hline 3 & conc. $\mathrm{HCl}(10.0$ equiv $) / \mathrm{MeOH} / \mathrm{rt} / 6 \mathrm{~h}$ & 18 \\
\hline 4 & conc. $\mathrm{HCl}(10.0$ equiv $) / \mathrm{MeOH} / \mathrm{reflux} / 6 \mathrm{~h}$ & 51 \\
\hline $\mathbf{5}$ & conc. $\mathbf{H C l}(\mathbf{2 0 . 0}$ equiv)/MeOH/reflux/12 h & $\mathbf{7 5}$ \\
\hline
\end{tabular}

${ }^{a}$ Isolated yields after column chromatography; NR = No Reaction.

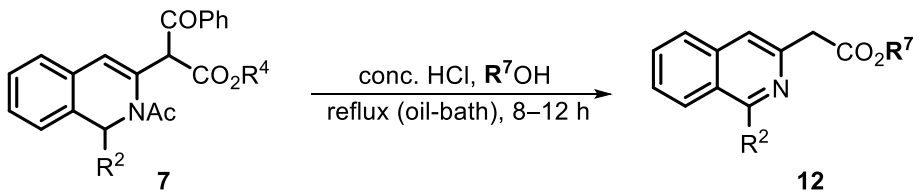

C(3)-alkylated product 7 (0.03 mmol) was dissolved in $\mathrm{MeOH}$ (2 $\mathrm{mL}$ or other protic solvent such as $\left.\mathrm{EtOH},{ }^{i} \mathrm{PrOH}\right)$ in a round bottom flask. Conc. $\mathrm{HCl}$ solution $(20 \mu \mathrm{L}, 20.0$ equiv) was then added to acidify the solution and the resulting mixture was refluxed for $8-12 \mathrm{~h}$ using a preheated oil bath. The progress of the reaction was checked by TLC. Upon completion of the reaction and subsequent cooling to room temperature, the reaction mixture was quenched with water and extracted with EtOAc $(2 \times 5 \mathrm{~mL})$. The organic extract was washed with water, brine and dried over anhydrous $\mathrm{Na}_{2} \mathrm{SO}_{4}$. The solution was filtered, concentrated under reduced pressure and the crude product was purified by silica gel flash column chromatography (eluted with Petroleum ether: EtOAc) to afford the desired decarbonylated product 12.

\section{Analytical data:}

Methyl 2-(isoquinolin-3-yl)acetate (12a): Yield 75\% (4 mg), Physical appearance: yellow gel,<smiles>CC(=O)Cc1cc2ccccc2cn1</smiles>

TLC $R_{f} 0.50$ (4:1, Petroleum ether: EtOAc); ${ }^{1} \mathrm{H}$ NMR $\left(500 \mathrm{MHz}, \mathrm{CDCl}_{3}\right) \delta$ $9.23(\mathrm{~s}, 1 \mathrm{H}), 7.97(\mathrm{dd}, \mathrm{J}=8.2,1.1 \mathrm{~Hz}, 1 \mathrm{H}), 7.80(\mathrm{~d}, \mathrm{~J}=8.2 \mathrm{~Hz}, 1 \mathrm{H}), 7.72$

- $7.67(\mathrm{~m}, 1 \mathrm{H}), 7.65(\mathrm{~s}, 1 \mathrm{H}), 7.61-7.56(\mathrm{~m}, 1 \mathrm{H}), 4.01(\mathrm{~s}, 2 \mathrm{H}), 3.75(\mathrm{~s}, 3 \mathrm{H}) ;{ }^{13} \mathrm{C}$ NMR $(176$ $\left.\mathrm{MHz}_{\mathrm{CDCl}}\right) \delta 171.4,152.4,147.5,136.4,130.6,127.5,127.5,127.1,126.3,120.3,120.0,53.4$, 
52.2, 43.4; IR (KBr, cm $\left.{ }^{-1}\right): 3410,2981,2365,1730,1628,1441,1260,990,750$; HRMS (ESI) $m / z:[\mathrm{M}+\mathrm{H}]^{+}$calcd. for $\mathrm{C}_{12} \mathrm{H}_{11} \mathrm{NO}_{2} 202.0863$; found 202.0866 .

Ethyl 2-(isoquinolin-3-yl)acetate (12b): Yield 72\% (4 mg), Physical appearance: colorless gel,

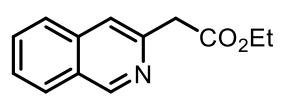

TLC $R_{f} 0.50$ (4:1, Petroleum ether: EtOAc); ${ }^{1} \mathbf{H}$ NMR (400 $\left.\mathrm{MHz} \mathrm{CDCl}_{3}\right) \delta$ $9.23(\mathrm{~s}, 1 \mathrm{H}), 7.96(\mathrm{~d}, J=8.1 \mathrm{~Hz}, 1 \mathrm{H}), 7.79(\mathrm{~d}, J=8.3 \mathrm{~Hz}, 1 \mathrm{H}), 7.71-7.66$ (m, 1H), $7.64(\mathrm{~s}, 1 \mathrm{H}), 7.60-7.56(\mathrm{~m}, 1 \mathrm{H}), 4.21(\mathrm{q}, J=7.1 \mathrm{~Hz}, 2 \mathrm{H}), 3.99(\mathrm{~s}, 2 \mathrm{H}), 1.27$ (t, $J=7.1$ $\mathrm{Hz}, 3 \mathrm{H}) .{ }^{13} \mathbf{C}$ NMR $\left(126 \mathrm{MHz}, \mathrm{CDCl}_{3}\right): \delta 171.0,152.3,147.8,136.4,130.5,127.5,127.0,126.3$, 119.9, 61.0, 43.6, 14.2; IR (KBr, cm $\left.{ }^{-1}\right): 3310,2872,1750,1610,1264,1186,1035,891,761$; HRMS (ESI) $m / z:[\mathrm{M}+\mathrm{H}]^{+}$calcd. for $\mathrm{C}_{13} \mathrm{H}_{13} \mathrm{NO}_{2} 216.1019$; found 216.1025.

Isopropyl 2-(isoquinolin-3-yl)acetate (12c): Yield 72\% (6 mg), Physical appearance: pale( $\mathrm{CO}_{2}{ }^{i \mathrm{Pr}}$ yellow gel, TLC $R_{f} 0.50$ (4:1, Petroleum ether: EtOAc); ${ }^{1} \mathbf{H}$ NMR $(400 \mathrm{MHz}$, $\left.\mathrm{CDCl}_{3}\right): \delta 9.22(\mathrm{~s}, 1 \mathrm{H}), 7.96(\mathrm{~d}, J=8.2 \mathrm{~Hz}, 1 \mathrm{H}), 7.79(\mathrm{~d}, J=8.2 \mathrm{~Hz}, 1 \mathrm{H}), 7.70$ - $7.66(\mathrm{~m}, 1 \mathrm{H}), 7.64(\mathrm{~s}, 1 \mathrm{H}), 7.60-7.56(\mathrm{~m}, 1 \mathrm{H}), 5.09(\mathrm{~m}, 1 \mathrm{H}), 3.96(\mathrm{~s}, 2 \mathrm{H}), 1.26(\mathrm{~d}, J=6.3 \mathrm{~Hz}$, 6H); ${ }^{13} \mathrm{C}$ NMR $\left(126 \mathrm{MHz}, \mathrm{CDCl}_{3}\right): \delta$ 170.6, 152.3, 147.9, 136.4, 130.4, 127.5, 127.4, 127.0, 126.3, 119.8, 68.4, 43.9, 21.8; IR (KBr, cm $\left.{ }^{-1}\right): 3390,2927,1781,1609,1438,1289,1096,833$, 779; HRMS (ESI) m/z: [M + H] $]^{+}$calcd. for $\mathrm{C}_{14} \mathrm{H}_{15} \mathrm{NO}_{2} 230.1176$; found 230.1189.

Methyl 2-(1-methylisoquinolin-3-yl)acetate (12d): Yield 70\% (7 mg), Physical appearance:

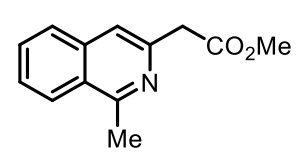
colorless solid, M.P. $70-72{ }^{\circ} \mathrm{C}$, TLC $R_{f} 0.50$ (4:1, Petroleum ether: EtOAc); ${ }^{1} \mathbf{H}$ NMR $\left(400 \mathrm{MHz}_{\mathrm{CDCl}}\right) \delta 8.10(\mathrm{~d}, J=8.3 \mathrm{~Hz}, 1 \mathrm{H}), 7.77(\mathrm{~d}, J=8.1 \mathrm{~Hz}$, 1H), $7.69-7.62(\mathrm{~m}, 1 \mathrm{H}), 7.60-7.53(\mathrm{~m}, 1 \mathrm{H}), 7.49(\mathrm{~s}, 1 \mathrm{H}), 3.96(\mathrm{~s}, 2 \mathrm{H}), 3.74$ (s, 3H), 2.95 (s, 3H). ${ }^{13} \mathbf{C}$ NMR (126 MHz, $\left.\mathrm{CDCl}_{3}\right): \delta$ 171.6, 158.7, 146.3, 136.4, 130.1, 127.0, 126.8, 126.3, 125.5, 118.6, 52.1, 43.4, 22.3; IR (KBr, $\left.\mathrm{cm}^{-1}\right): 3406,2925,2352,1721,1601$, 1423, 1289, 763, 733; HRMS (ESI) $m / z:[\mathrm{M}+\mathrm{H}]^{+}$calcd. for $\mathrm{C}_{13} \mathrm{H}_{13} \mathrm{NO}_{2}$ 216.1019; found 216.1025 .

\section{Scheme S15: Selective Decarboxylation:}

Table S4: Optimization of reaction conditions for decarboxylation:

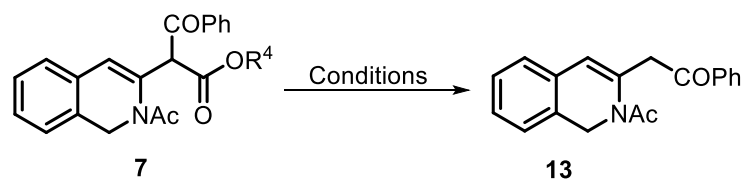




\begin{tabular}{|c|c|c|c|}
\hline Entry & $\mathbf{R}^{4}$ & Conditions & Yield of $13(\%)^{a}$ \\
\hline 1 & $\mathrm{Et}$ & $\mathrm{LiOH}\left(4.0\right.$ equiv)/THF/ $\mathrm{H}_{2} \mathrm{O}(1: 1) / \mathrm{rt} / 6 \mathrm{~h}$ & NR \\
\hline 2 & $\mathrm{Et}$ & $\mathrm{LiOH}\left(4.0\right.$ equiv)/THF/ $\mathrm{H}_{2} \mathrm{O}(1: 1) / 60^{\circ} \mathrm{C} / 6 \mathrm{~h}$ & NR \\
\hline 3 & $\mathrm{Et}$ & $\mathrm{LiOH}\left(4.0\right.$ equiv)/MeOH/ $/ \mathrm{H}_{2} \mathrm{O}(5: 1) / \mathrm{rt} / 6 \mathrm{~h}$ & NR \\
\hline 4 & $\mathrm{Et}$ & $\mathrm{K}_{2} \mathrm{CO}_{3}\left(4.0\right.$ equiv)/MeOH$/ 60{ }^{\circ} \mathrm{C} / 8 \mathrm{~h}$ & $\begin{array}{c}\text { Compound } \mathbf{X} \text { obtained } \\
\text { (shown below) }\end{array}$ \\
\hline 5 & $\mathrm{Et}$ & $\mathrm{LiCl}\left(5.0\right.$ equiv)/DMSO/ $\mathrm{H}_{2} \mathrm{O}(4: 1) / 120^{\circ} \mathrm{C} / 8 \mathrm{~h}$ & NR \\
\hline 6 & $\mathrm{Et}$ & $\mathrm{NaCl}\left(1.5\right.$ equiv)/DMSO/ $\mathrm{H}_{2} \mathrm{O}(4: 1) / 100^{\circ} \mathrm{C} / 8 \mathrm{~h}$ & NR \\
\hline 7 & $\mathrm{Et}$ & $\mathrm{LiI}\left(1.5\right.$ equiv)/DMSO/ $\mathrm{H}_{2} \mathrm{O}(4: 1) / 100^{\circ} \mathrm{C} / 8 \mathrm{~h}$ & NR \\
\hline 8 & $\mathrm{Et}$ & $\mathrm{KOH}(4.0$ equiv $) / \mathrm{EtOH} / \mathrm{H}_{2} \mathrm{O}(5: 1) / \mathrm{rt} / 6 \mathrm{~h}$ & Complex mixture \\
\hline 9 & Et & $6 \mathrm{M} \mathrm{HCl} /$ dioxane $/ 60^{\circ} \mathrm{C} / 8 \mathrm{~h}$ & NR \\
\hline 10 & Bn & $10 \mathrm{~mol} \% \mathrm{Pd} / \mathrm{C} / \mathrm{H}_{2}(1 \mathrm{~atm}) / \mathrm{EtOH} / \mathrm{rt} / 4 \mathrm{~h}$ & 89 \\
\hline 11 & $\mathrm{Bn}$ & $10 \mathrm{~mol} \% \mathrm{Pd} / \mathrm{C} / \mathrm{H}_{2}(1 \mathrm{~atm}) / \mathrm{MeOH} / \mathrm{rt} / 4 \mathrm{~h}$ & 80 \\
\hline
\end{tabular}

${ }^{a}$ Isolated yields after column chromatography. NR = No Reaction.

Compound X: Methyl 2-(2-acetyl-1,2-dihydroisoquinolin-3-yl)acetate:

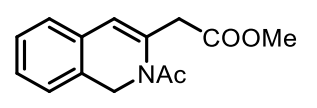

Yield: 31\% (2 mg), Physical appearance: pale-yellow oil, TLC $R_{f} 0.40$ (7:3, Petroleum ether: EtOAc); ${ }^{1} \mathbf{H}$ NMR $\left(400 \mathrm{MHz}, \mathrm{CDCl}_{3}\right) \delta 7.25-7.23(\mathrm{dd}, J$ $=7.4,1.5 \mathrm{~Hz}, 1 \mathrm{H}), 7.22-7.18(\mathrm{~m}, 1 \mathrm{H}), 7.14-7.10(\mathrm{~m}, 2 \mathrm{H}), 6.24(\mathrm{~s}, 1 \mathrm{H}), 4.67(\mathrm{~s}, 2 \mathrm{H}), 3.78(\mathrm{br}$ s, 2H), $3.72(\mathrm{~s}, 3 \mathrm{H}), 2.24(\mathrm{~s}, 3 \mathrm{H})$; HRMS (ESI) $\mathrm{m} / z$ : $[\mathrm{M}+\mathrm{H}]^{+}$calcd. for $\mathrm{C}_{14} \mathrm{H}_{15} \mathrm{NO}_{3} 246.1125$; found 246.1138 .

\section{Procedure for decarboxylation:}

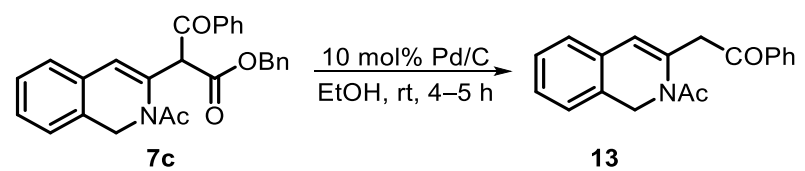

To a stirred solution of substituted benzyl 2-(2-acetyl-1,2-dihydroisoquinolin-3-yl)-3-oxo-3phenylpropanoate $(10 \mathrm{mg}, 0.023 \mathrm{mmol})$ in $\mathrm{EtOH}(2 \mathrm{~mL})$ was added $10 \% \mathrm{Pd} / \mathrm{C}(2 \mathrm{mg}, 10 \mathrm{~mol} \%)$ at room temperature. The reaction mixture was stirred at room temperature under $\mathrm{H}_{2}$ balloon for 4-5 h. The progress of the reaction was monitored by TLC, and upon completion of the reaction, the solution was degassed with nitrogen and filtered through a pad of Celite, and the filtrate was concentrated under reduced pressure. The crude product was purified by silica gel flash column 
chromatography (eluting with Petroleum ether: EtOAc (7:3)) to yield the desired decarboxylated product 13 .

2-(2-acetyl-1,2-dihydroisoquinolin-3-yl)-1-phenylethan-1-one (13): Yield $89 \%$ (4 mg),

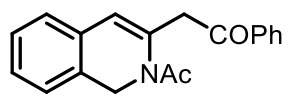
Physical appearance: colorless gel, TLC $R_{f} 0.50$ (4:1, Petroleum ether: EtOAc); ${ }^{1} \mathbf{H}$ NMR (400 MHz, $\left.\mathrm{CDCl}_{3}\right) \delta 8.04$ - 7.98 (m, 2H), 7.61 - 7.54 (m, 1H), $7.47(\mathrm{t}, J=7.6 \mathrm{~Hz}, 2 \mathrm{H}), 7.28-7.23(\mathrm{~m}, 2 \mathrm{H}), 7.23-7.18(\mathrm{~m}, 1 \mathrm{H}), 7.17-7.13(\mathrm{~m}, 1 \mathrm{H}), 7.12$ $(\mathrm{dd}, J=7.2,1.5 \mathrm{~Hz}, 1 \mathrm{H}), 6.23(\mathrm{~s}, 1 \mathrm{H}), 4.72(\mathrm{~s}, 2 \mathrm{H}), 4.53(\mathrm{~s}, 2 \mathrm{H}), 2.21(\mathrm{~s}, 3 \mathrm{H}) ;{ }^{13} \mathbf{C}$ NMR $(176$ $\left.\mathrm{MHz}_{\mathrm{CDCl}}\right): \delta 196.8,169.5,136.6,136.4,132.0,131.2,128.6,128.2,128.0,127.2,124.8$, 124.5, 119.9, 50.0, 43.8, 23.1; IR (KBr, $\left.\mathrm{cm}^{-1}\right): 3065,2981,1734,1662,1397,1265,1159$; HRMS (ESI) $m / z[\mathrm{M}+\mathrm{H}]^{+}$: calcd. for $\mathrm{C}_{19} \mathrm{H}_{17} \mathrm{NO}_{2} 292.1332$, found 292.1341.

vi) General method for large scale transformation $(2 \mathrm{mmol})$ of substrate $1 \mathrm{c}$ :

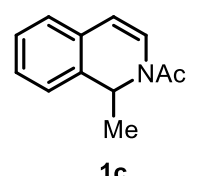

$1 \mathrm{c}$

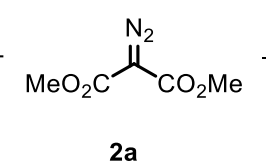

$\underset{60^{\circ} \mathrm{C} \text { (oil-bath), HFIP, } 4 \mathrm{~h}}{\frac{\left[\mathrm{Cp}^{*} \mathrm{IrCl}_{2}\right]_{2}, \mathrm{AgBF}_{4}, \text { Boc-Pro-OH }}{\longrightarrow}}$

$2 \mathbf{a}$

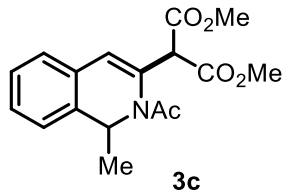

In a pressure tube equipped with a stir bar, 1-(1-methylisoquinolin-2(1H)-yl)ethan-1-one 1c (374 mg, $2.0 \mathrm{mmol}$ ), dimethyl 2-diazomalonate $\mathbf{2 a}(379.2 \mathrm{mg}, 2.4 \mathrm{mmol}),\left[\mathrm{Cp}^{*} \mathrm{IrCl}_{2}\right]_{2}(48 \mathrm{mg}, 3$ mol\%), $\mathrm{AgBF}_{4}(48 \mathrm{mg}, 12 \mathrm{~mol} \%$ ) and Boc-Pro-OH (86 mg, 0.2 equiv) were added simultaneously under argon flow. This was followed by the addition of distilled HFIP (20.0 mL). The reaction tube was sealed and immersed in an oil bath preheated to $60{ }^{\circ} \mathrm{C}$, with stirring. Thereafter, progress of the reaction was checked by TLC. Upon completion, the reaction mixture was cooled to room temperature, filtered through a pad of Celite and eluted with EtOAc. The filtrate was washed with brine, and dried over anhydrous $\mathrm{Na}_{2} \mathrm{SO}_{4}$. After concentration of the organic extract under reduced pressure, the crude product was purified by a silica gel flash column chromatography to yield the desired C(3)-alkylated product 3c in 62\% (393 mg) yield.

\section{vii) Control experiments and mechanistic studies:}

\section{Scheme S16: Control Experiments:}

A) i) Control reaction with cyclohexene: In presence of $\mathrm{N}$-acetyl-1,2-dihydroisoquinoline (4A): 

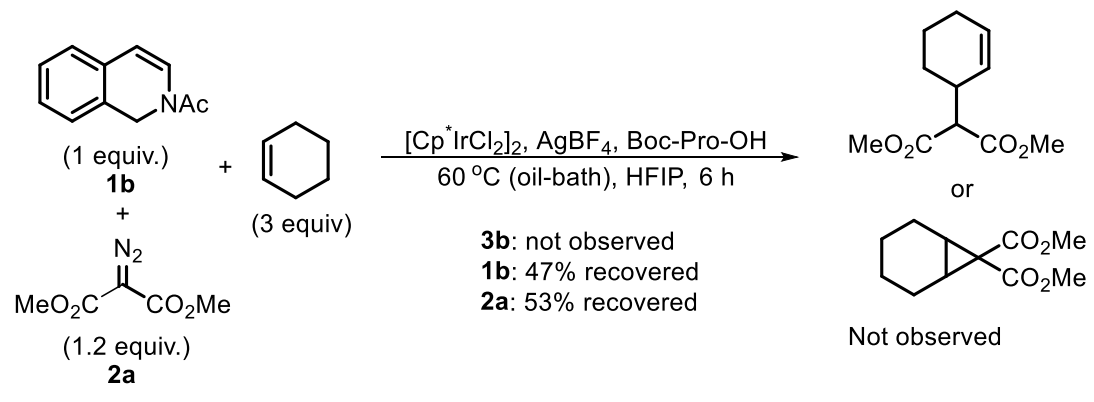

In a pressure tube equipped with a stir bar, $N$-acetyl-1,2-dihydroisoquinoline $\mathbf{1 b}(0.1 \mathrm{mmol})$, dimethyl 2-diazomalonate $2 \mathbf{2 a}(0.12 \mathrm{mmol})$ and cyclohexene $(0.3 \mathrm{mmol})$ were added simultaneously. This was followed by the addition of $\left[\mathrm{Cp}^{*} \mathrm{IrCl}_{2}\right]_{2}(2.4 \mathrm{mg}, 3 \mathrm{~mol} \%), \mathrm{AgBF}_{4}(2.4$ $\mathrm{mg}, 12 \mathrm{~mol} \%)$ and Boc-Pro-OH (4.3 mg, 0.2 equiv) under an argon flow. Distilled HFIP (1.0 $\mathrm{mL}$ ) was subsequently added, and the reaction tube was sealed and immersed into an oil bath pre-heated to $60{ }^{\circ} \mathrm{C}$, with stirring. After 6 hours, the reaction mixture was cooled to room temperature, filtered through a pad of Celite and eluted with EtOAc. The filtrate was washed with brine and dried over anhydrous $\mathrm{Na}_{2} \mathrm{SO}_{4}$. After concentration of the organic extract under reduced pressure, the crude mixture was analysed by ${ }^{1} \mathrm{H}$ NMR which showed that cyclopropanation or allylic $\mathrm{C}-\mathrm{H}$ insertion of the cyclohexene did not occur and formation of $\mathbf{3 b}$ was completely inhibited. $\mathbf{1 b}$ and $\mathbf{2 a}$ was recovered in $47 \%$ and $53 \%$ yields, respectively, after silica gel flash column chromatography (eluted with 7:3, Petroleum ether: EtOAc).

\section{ii) Control reaction with cyclohexene: In absence of $N$-acetyl-1,2-dihydroisoquinoline (4A):}

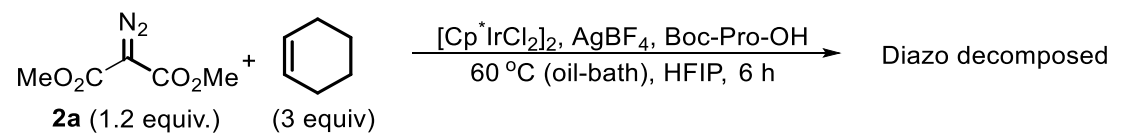

In a pressure tube equipped with a stir bar, dimethyl 2-diazomalonate $\mathbf{2 a}(0.12 \mathrm{mmol})$ and cyclohexene $(0.3 \mathrm{mmol})$ were added simultaneously. This was followed by the addition of $\left[\mathrm{Cp}^{*} \mathrm{IrCl}_{2}\right]_{2}(2.4 \mathrm{mg}, 3 \mathrm{~mol} \%), \mathrm{AgBF}_{4}(2.4 \mathrm{mg}, 12 \mathrm{~mol} \%$ ) and Boc-Pro-OH (4.3 mg, 0.2 equiv) under an argon flow. Distilled HFIP $(1.0 \mathrm{~mL})$ was subsequently added, and the reaction tube was sealed and immersed into an oil bath pre-heated to $60{ }^{\circ} \mathrm{C}$, with stirring. After 6 hours, the reaction mixture was cooled to room temperature, filtered through a pad of Celite and eluted with EtOAc. TLC indicated complete decomposition of the diazo coupling partner. No cyclopropanation or allylic $\mathrm{C}-\mathrm{H}$ insertion on the cyclohexene was observed by crude ${ }^{1} \mathrm{H}$ NMR analysis. 
B) i) Intermolecular competition reaction: In presence of $N$-acetyl-1,2-dihydroisoquinoline (4B):

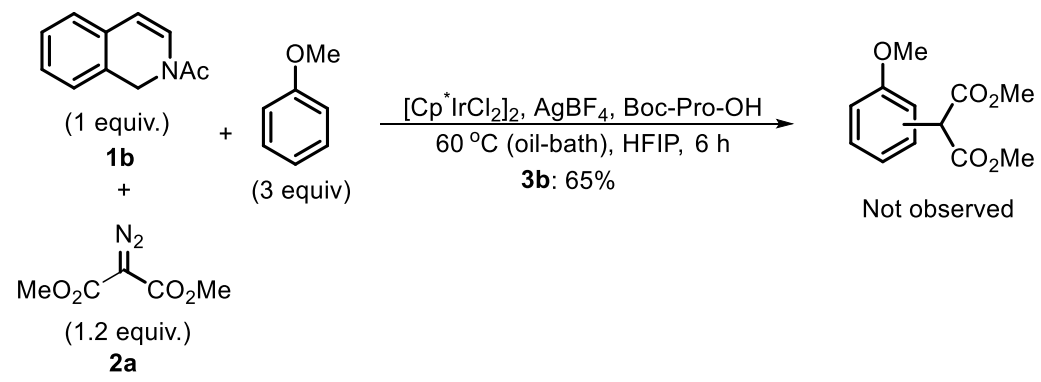

In a pressure tube equipped with a stir bar, $N$-acetyl-1,2-dihydroisoquinoline $\mathbf{1 b}(0.1 \mathrm{mmol})$, dimethyl 2-diazomalonate $\mathbf{2 a}(0.12 \mathrm{mmol})$ and anisole $(0.3 \mathrm{mmol})$ was added simultaneously. This was followed by the addition of $\left[\mathrm{Cp}^{*} \mathrm{IrCl}_{2}\right]_{2}$ (2.4 mg, $\left.3 \mathrm{~mol} \%\right), \mathrm{AgBF}_{4}(2.4 \mathrm{mg}, 12 \mathrm{~mol} \%$ ) and Boc-Pro-OH (4.3 mg, 0.2 equiv) under an argon flow. Distilled HFIP (1.0 mL) was subsequently added, and the reaction tube was sealed and immersed into an oil bath pre-heated to $60{ }^{\circ} \mathrm{C}$, with stirring. After 6 hours, the reaction mixture was cooled to room temperature, filtered through a pad of Celite and eluted with EtOAc. The filtrate was washed with brine and dried over anhydrous $\mathrm{Na}_{2} \mathrm{SO}_{4}$. After concentration of the organic extract under reduced pressure, the crude product was purified by a silica gel flash column chromatography (eluting with 7:3, Petroleum ether: EtOAc). ${ }^{1} \mathrm{H}$ NMR analysis showed formation of $\mathrm{C}-\mathrm{H}$ alkylation product $\mathbf{3 b}$ in $65 \%$ yield and the $\mathrm{C}-\mathrm{H}$ insertion product of the electron-rich anisole with diazo compound $2 \mathbf{a}$ was not observed.

\section{ii) Intermolecular competition reactions: In presence of $\mathbf{N}$-acetyl-1,2-dihydroisoquinoline} (4B):
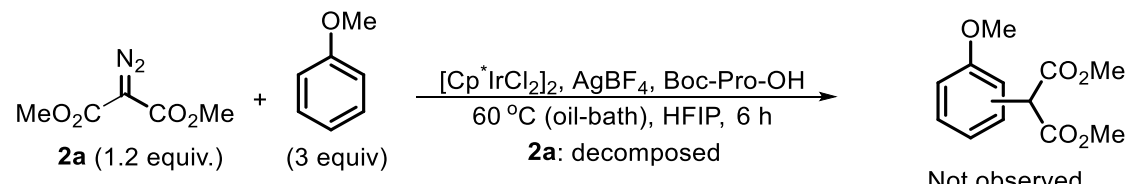

In a pressure tube equipped with a stir bar, dimethyl 2-diazomalonate $2 \mathbf{a}(0.12 \mathrm{mmol})$ and anisole $(0.3 \mathrm{mmol})$ were added simultaneously. This was followed by the addition of $\left[\mathrm{Cp}^{*} \mathrm{IrCl}_{2}\right]_{2}$ (2.4 mg, $3 \mathrm{~mol} \%$ ), $\mathrm{AgBF}_{4}(2.4 \mathrm{mg}, 12 \mathrm{~mol} \%$ ) and Boc-Pro-OH (4.3 mg, 0.2 equiv) under an argon flow. Distilled HFIP (1.0 mL) was subsequently added, and the reaction tube was sealed and immersed into an oil bath pre-heated to $60{ }^{\circ} \mathrm{C}$, with stirring. After 6 hours, the reaction mixture was cooled to room temperature. A TLC analysis showed complete decomposition of the diazo coupling partner. No $\mathrm{C}-\mathrm{H}$ insertion product was observed. 


\section{C) Reaction to test for the site-selectivity of alkylation (4C):}

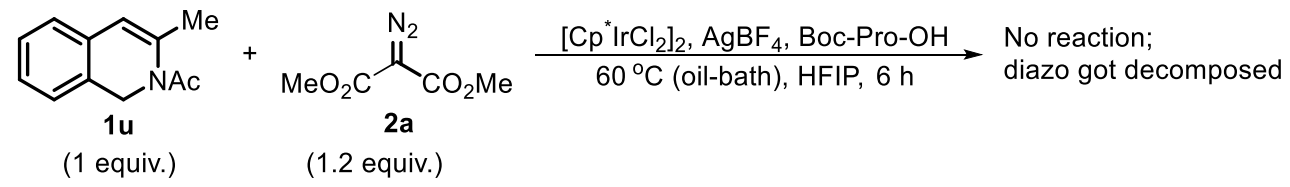

In a pressure tube equipped with a stir bar, 1-(3-methylisoquinolin-2(1H)-yl)ethan-1-one 1u $(0.1$ mmol), dimethyl 2-diazomalonate $2 \mathbf{a}(0.12 \mathrm{mmol}),\left[\mathrm{Cp}^{*} \mathrm{IrCl}_{2}\right]_{2}(2.4 \mathrm{mg}, 3 \mathrm{~mol} \%), \mathrm{AgBF}_{4}(2.4$ $\mathrm{mg}, 12 \mathrm{~mol} \%)$ and Boc-Pro-OH (4.3 mg, 0.2 equiv) were added simultaneously under argon flow. Distilled HFIP (1.0 mL) was subsequently added, and the reaction tube was sealed and immersed into an oil bath pre-heated to $60^{\circ} \mathrm{C}$, with stirring. TLC indicated no product formation even after $6 \mathrm{~h}$ with the starting material (1u) remaining unreacted. The reaction mixture was cooled to room temperature, filtered through a pad of Celite and eluted with EtOAc. The filtrate was washed with brine and dried over anhydrous $\mathrm{Na}_{2} \mathrm{SO}_{4}$. After concentration of the organic extract under reduced pressure, the crude product was purified by silica gel flash column chromatography (eluting with 7:3, Petroleum ether: EtOAc). No C(3)-alkylation product was formed and the starting material (1u) was recovered back along with the decomposition of diazo compound.

\section{D. (i) Reversibility study in C(3)-alkylation in absence of coupling partner (4D):}

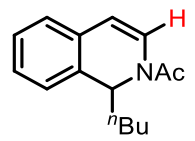

$1 f$

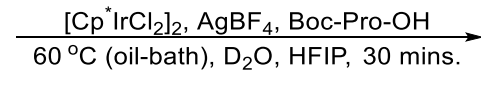

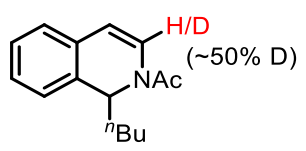

1f'

In a pressure tube equipped with a stir bar, 1-(1-butylisoquinolin-2(1H)-yl)ethan-1-one 1f $(0.1$ $\mathrm{mmol}),\left[\mathrm{IrCp}^{*} \mathrm{Cl}_{2}\right]_{2}(2.4 \mathrm{mg}, 3 \mathrm{~mol} \%), \mathrm{AgBF}_{4}(2.4 \mathrm{mg}, 12 \mathrm{~mol} \%)$ and Boc-Pro-OH (4.3 mg, 0.2 equiv) were added simultaneously under argon flow. Distilled HFIP (1.0 mL) and $\mathrm{D}_{2} \mathrm{O}(1 \mathrm{mmol})$ were subsequently added, and the reaction tube was sealed and immersed into an oil bath preheated to $60{ }^{\circ} \mathrm{C}$, with stirring. After 30 minutes, the reaction mixture was cooled to room temperature, filtered through a pad of Celite and eluted with EtOAc. The filtrate was transferred to a separatory funnel and washed with brine solution. The organic extract was dried over anhydrous $\mathrm{Na}_{2} \mathrm{SO}_{4}$, filtered and concentrated under reduced pressure. The crude product was purified by a silica gel flash column chromatography (eluted with 7:3, Petroleum ether: EtOAc). 
The isolated compound was analyzed by ${ }^{1} \mathrm{H}$ NMR, which showed $\sim 50 \%$ deuterium incorporation at the $\mathrm{C}(3)-$ position.

\section{(ii) Reversibility study in C(3)-alkylation in presence of coupling partner:}

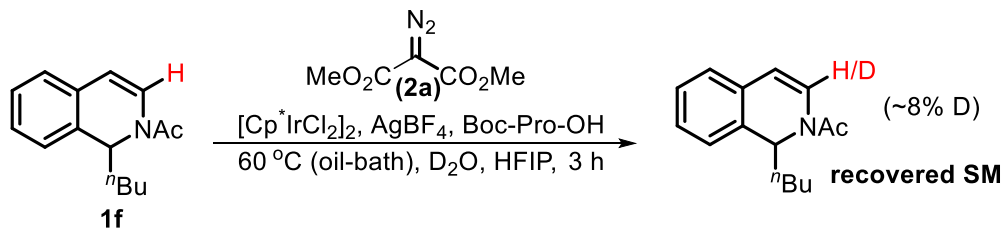

In a pressure tube equipped with a stir bar, $N$-acetyl-1,2-dihydroisoquinolines $1 \mathrm{a}(0.1 \mathrm{mmol})$, dimethyl 2-diazomalonate $\mathbf{2 a}(0.12 \mathrm{mmol})$, [ $\left.\mathrm{IrCp}^{*} \mathrm{Cl}_{2}\right]_{2}(2.4 \mathrm{mg}, 3 \mathrm{~mol} \%), \mathrm{AgBF}_{4}(2.4 \mathrm{mg}, 12$ $\mathrm{mol} \%$ ) and Boc-Pro-OH (4.3 mg, 0.2 equiv) were added simultaneously under argon flow. Distilled HFIP $(1.0 \mathrm{~mL})$ and $\mathrm{D}_{2} \mathrm{O}(1 \mathrm{mmol})$ were subsequently added, and the reaction tube was sealed and immersed into an oil bath pre-heated to $60{ }^{\circ} \mathrm{C}$, with stirring. The reaction was stirred for another 3 hours and was cooled to room temperature, filtered through a pad of Celite and eluted with EtOAc. The filtrate was transferred to a separatory funnel and washed with brine. The organic extract was dried over anhydrous $\mathrm{Na}_{2} \mathrm{SO}_{4}$, filtered and concentrated under reduced pressure. The crude product was purified by a silica gel flash column chromatography (eluting with 7:3, Petroleum ether: EtOAc). The isolated starting material was analyzed by ${ }^{1} \mathrm{H}$ NMR, indicating $\sim 8 \%$ deuteration at the $\mathrm{C}(3)$-position. This indicated that the steps following the $\mathrm{C}-\mathrm{H}$ activation may be faster than the reverse step.

\section{E. Mass spectrometry Study:}

In a pressure tube equipped with a stir bar, $N$-acetyl-1,2-dihydroisoquinolines $\mathbf{1 b}(0.1 \mathrm{mmol})$, dimethyl 2-diazomalonate $\mathbf{2 a}(0.12 \mathrm{mmol}),\left[\mathrm{IrCp}^{*} \mathrm{Cl}_{2}\right]_{2}(2.4 \mathrm{mg}, 3 \mathrm{~mol} \%), \mathrm{AgBF}_{4}(2.4 \mathrm{mg}, 12$ $\mathrm{mol} \%$ ) and Boc-Pro-OH (4.3 mg, 0.2 equiv) were added simultaneously under argon flow. Distilled HFIP (1.0 mL) was subsequently added, and the reaction tube was sealed and stirred at $60{ }^{\circ} \mathrm{C}$ (preheated oil bath). After 2 mins, the reaction mixture was subjected to mass analysis. Similarly for another reaction in which no coupling partner was added, same procedure was followed at room temperature. 


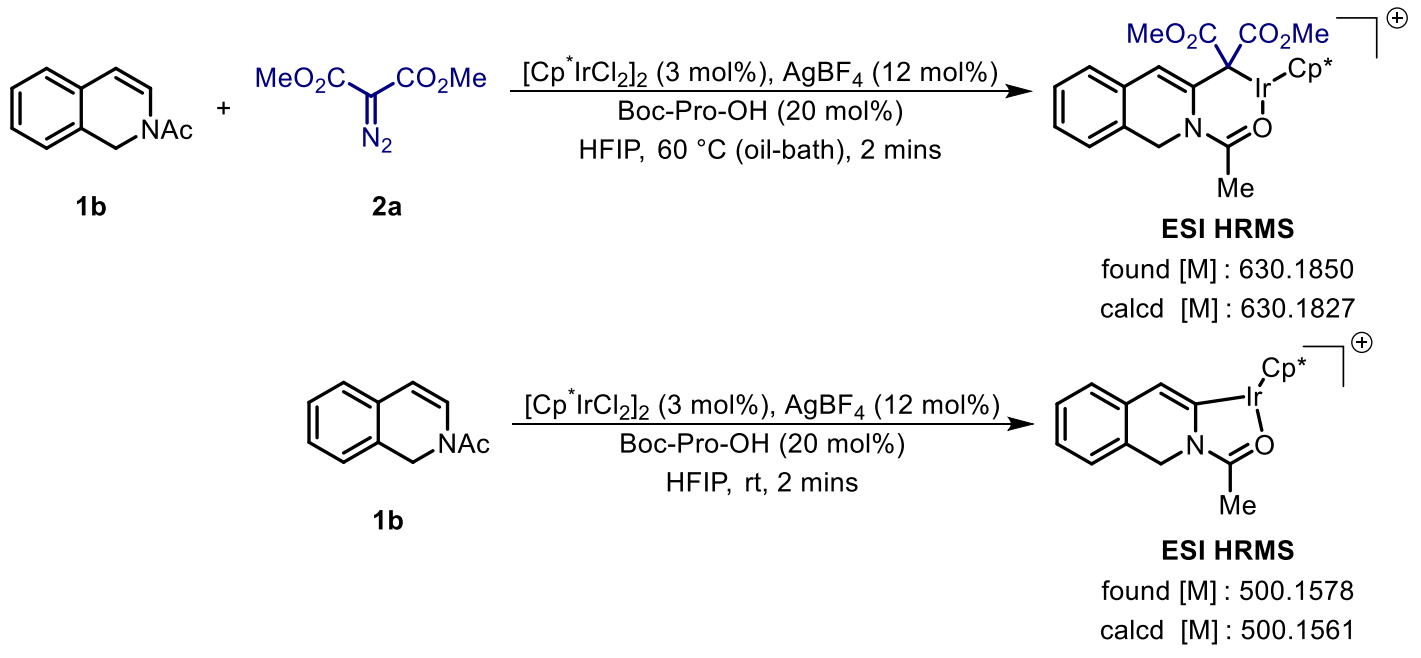

\section{References:}

(1) Tiwari,V. K.; Kamal, N.; Kapur, M. Org. Lett. 2017, 19, 262.

(2) (a) Jin, Y.; Makida,Y.; Uchida, T.; Kuwano, R. J. Org. Chem. 2018, 83, 3829. (b) Jakab, A.; Dalicsek, Z.; Holczbauer, T.; Hamza, T.; Pápai, I.; Finta, Z.; Timári, G.; Soós, T. Eur. J. Org. Chem. 2015, 2015, 60. (c) Das, R.; Khot, N. P.; Kapur, M. Synthesis. 2019, 51, 2515. (d) Choudhury, A. R.; Mukherjee, S. Chem. Sci. 2016, 7, 6940. (e) Cao, V. D.; Jo, D. G.; Kim, H.; Kim, C.; Yun, S.; Joung, S. Synthesis. 2021, 53, 754.

(3) Wu, Y.; Chen, Z.; Yang, Y.; Zhu, W.; Zhou, B. J. Am. Chem. Soc. 2018, 140, 42.

(4) Patel, P.; Borah, G. Chem. Commun. 2017, 53, 443.

(5) (a) Tang, G. -D.; Pan, C. -L.; Li, X. Org. Chem. Front. 2016, 3, 87. (b) Guo, S.; Sun, L.; Wang, F.; Zhang, X.; Fan, X. J. Org. Chem. 2018, 83, 12034. (c) Chen, X.; Zheng, G.; Li, Y.; Song, G.; Li, X. Org. Lett. 2017, 19, 6184.

(6) (a) Fang, S.; Zhao, Y.; Li, H.; Zheng,Y.; Lian, P.; Wan, X. Org. Lett. 2019, 21, 2356. (b) Jiang, Y.; Chen, X.; Zheng, Y.; Xue, Z.; Shu, C.; Yuan, W.; Zhang, X. Angew. Chem., Int. Ed. 2011, 50, 7304.

(7) Tsai, Y. -T.; Zhu, J. -T. J. Org. Chem. 2021, 86, 813.

(8) Hu, W.; He, X.; Zhou, T.; Zuo, Y.; Zhang, S.; Yang, T.; Shang, Y. Org. Biomol. Chem. 2021, 19, 552. 


\section{Copies of ${ }^{1} \mathrm{H}$ and ${ }^{13} \mathrm{C}$ NMR spectra:}
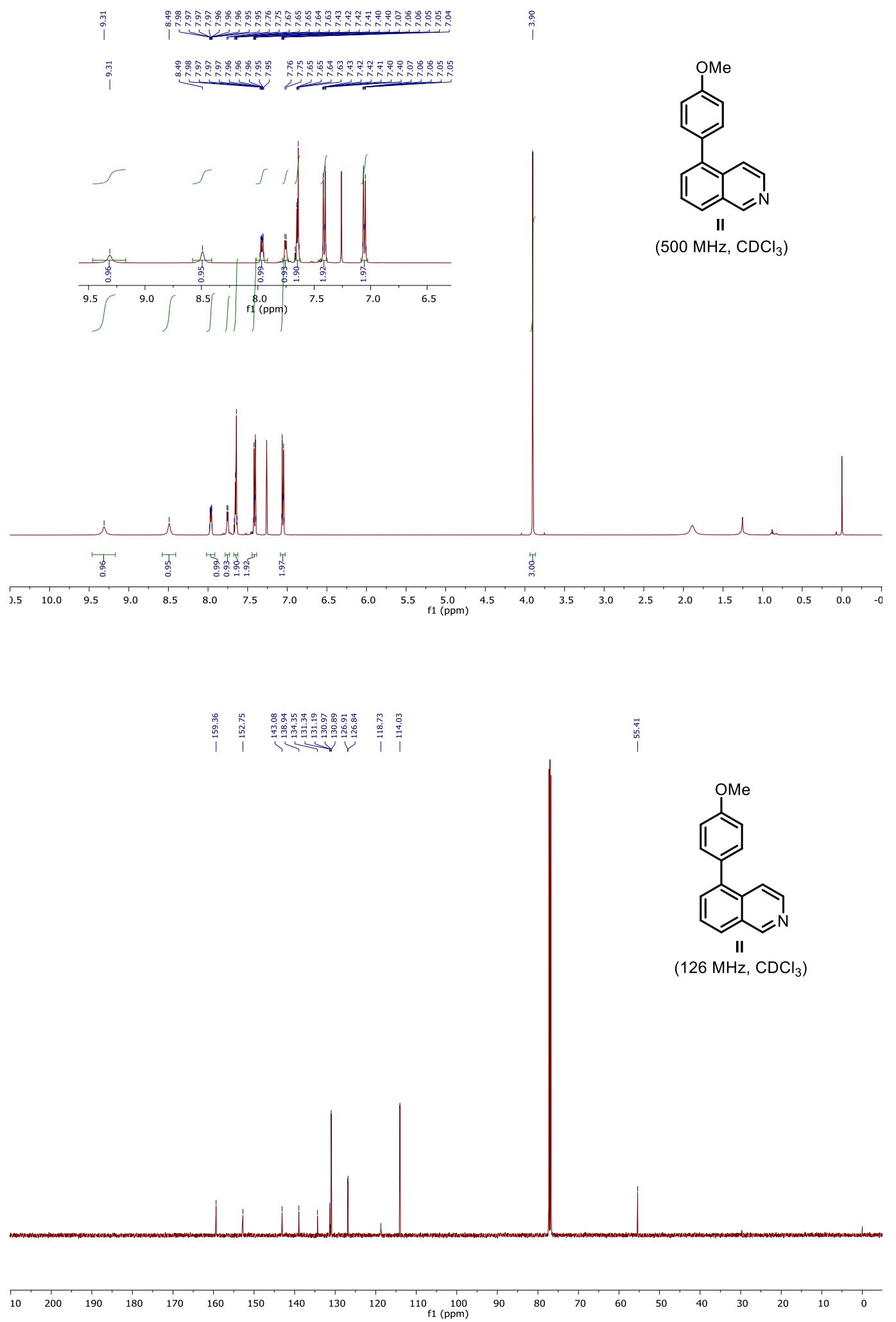


\section{啚}
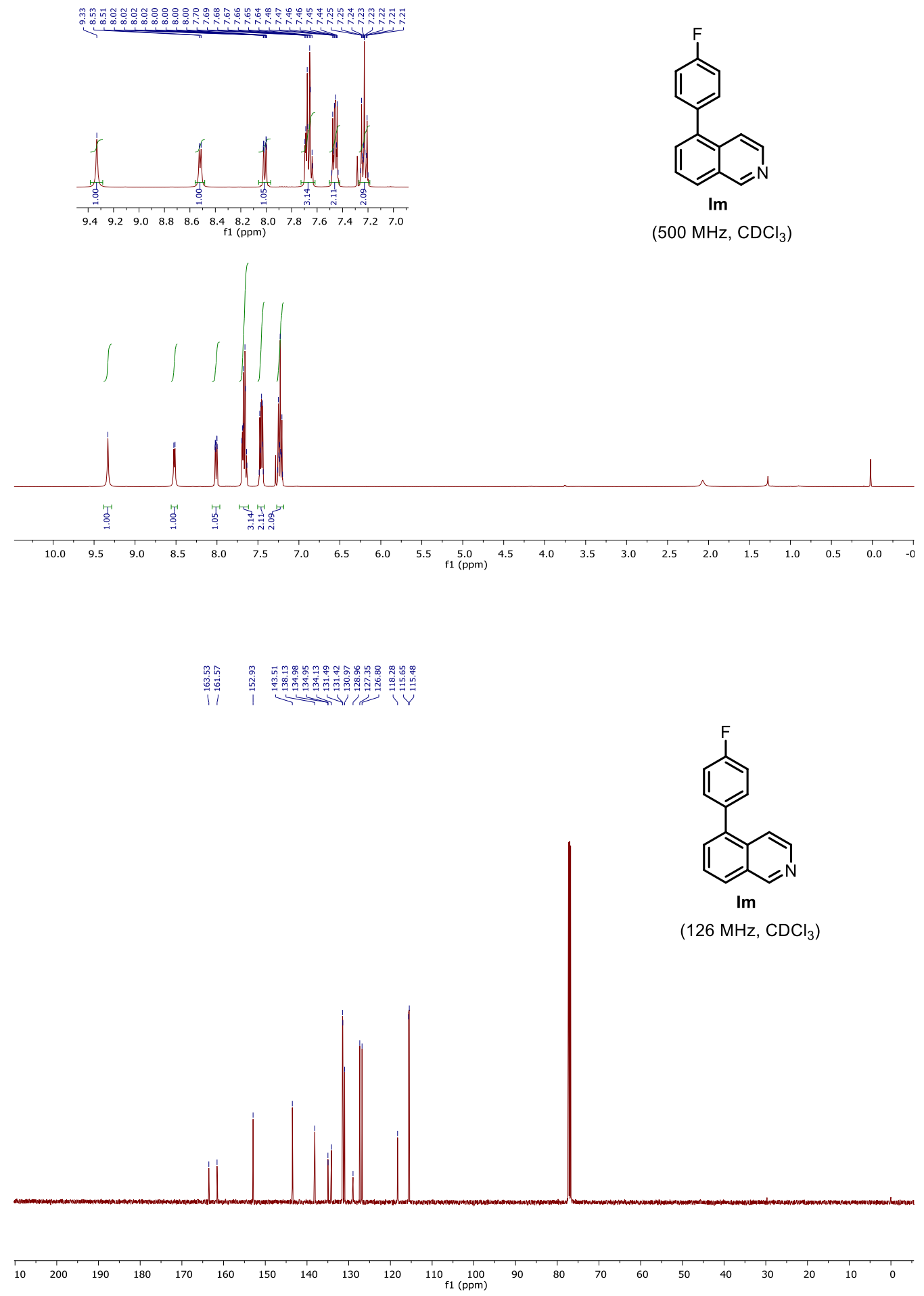


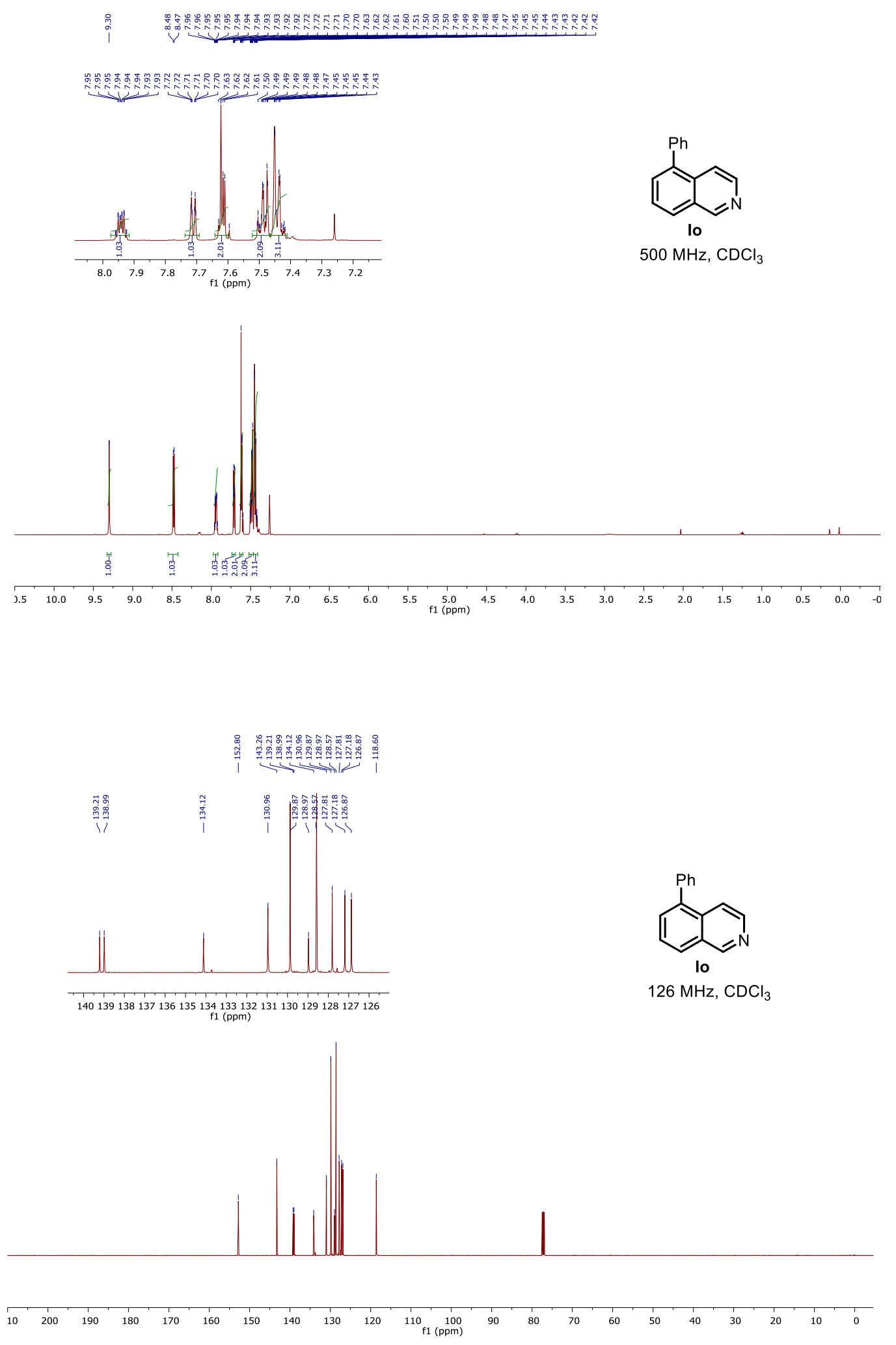


前

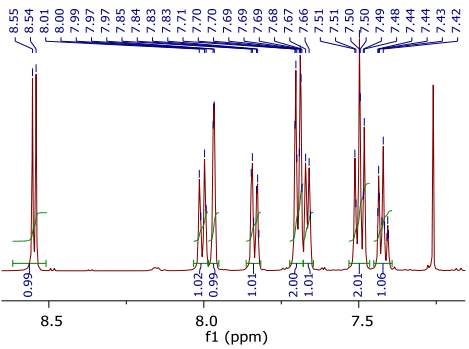

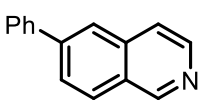

Ip

(500 MHz, $\mathrm{CDCl}_{3}$ )

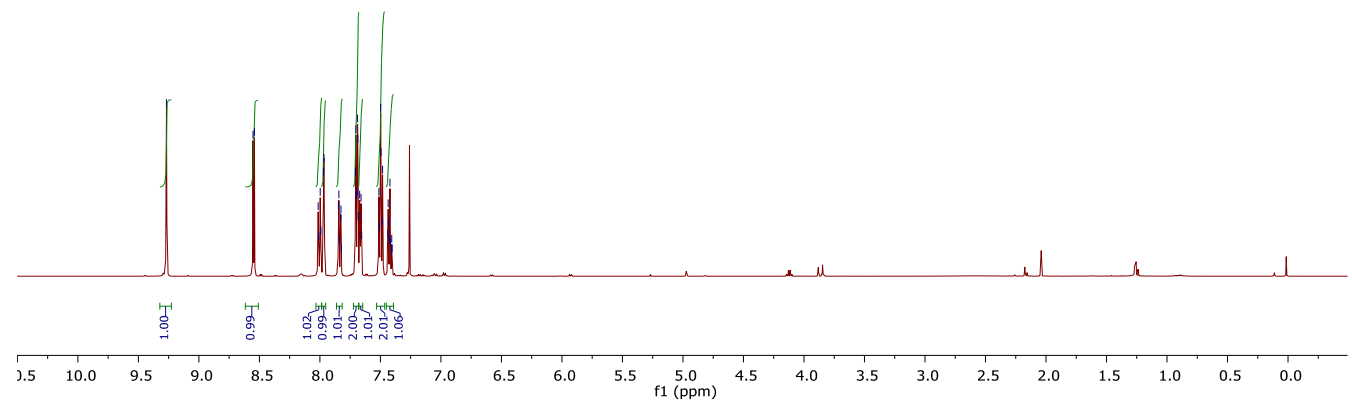

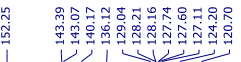
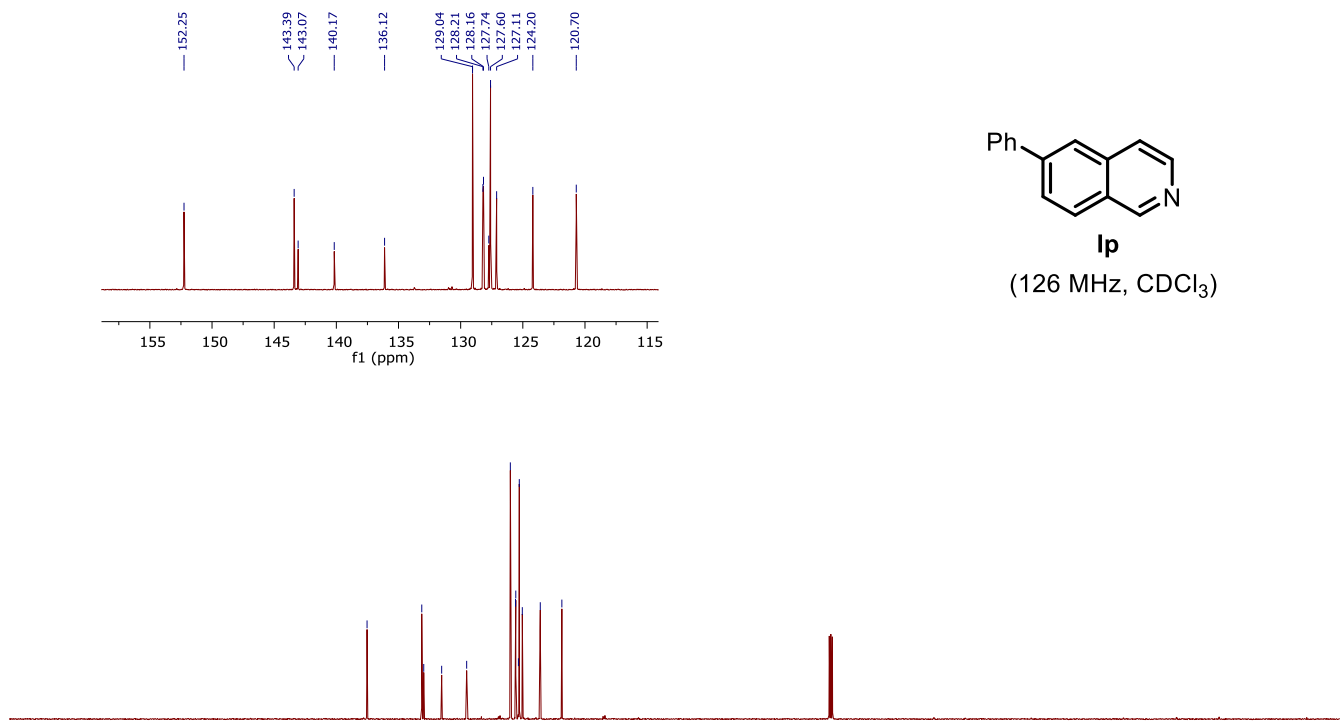

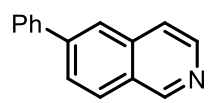

Ip

$\left(126 \mathrm{MHz}, \mathrm{CDCl}_{3}\right.$ )

10

$\begin{array}{lllll}180 & 170 & 160 & 150\end{array}$

$140 \quad 130$

$110 \begin{array}{r}100 \\ \text { f1 }(\mathrm{ppm})\end{array}$ 


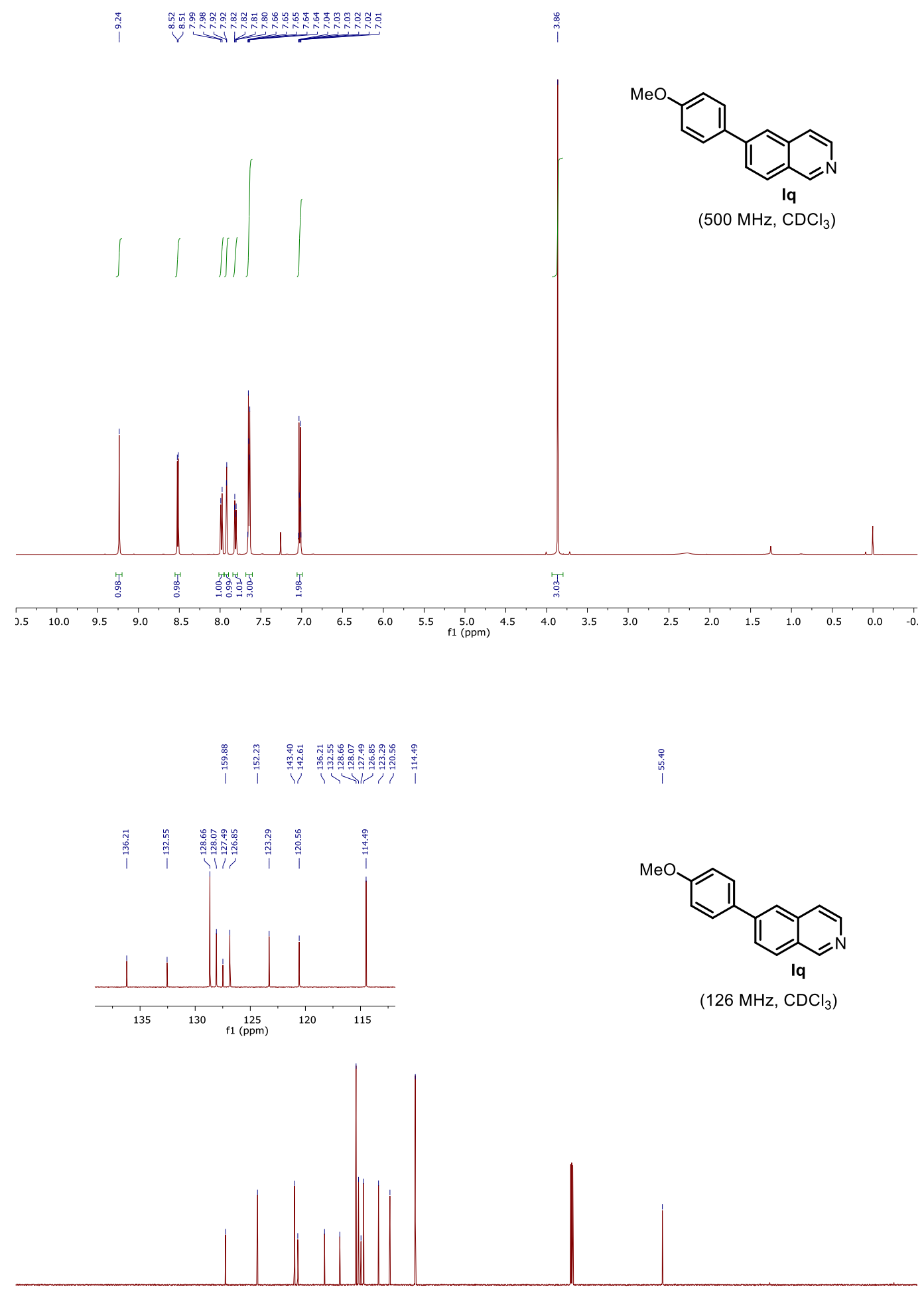

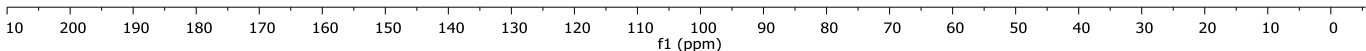




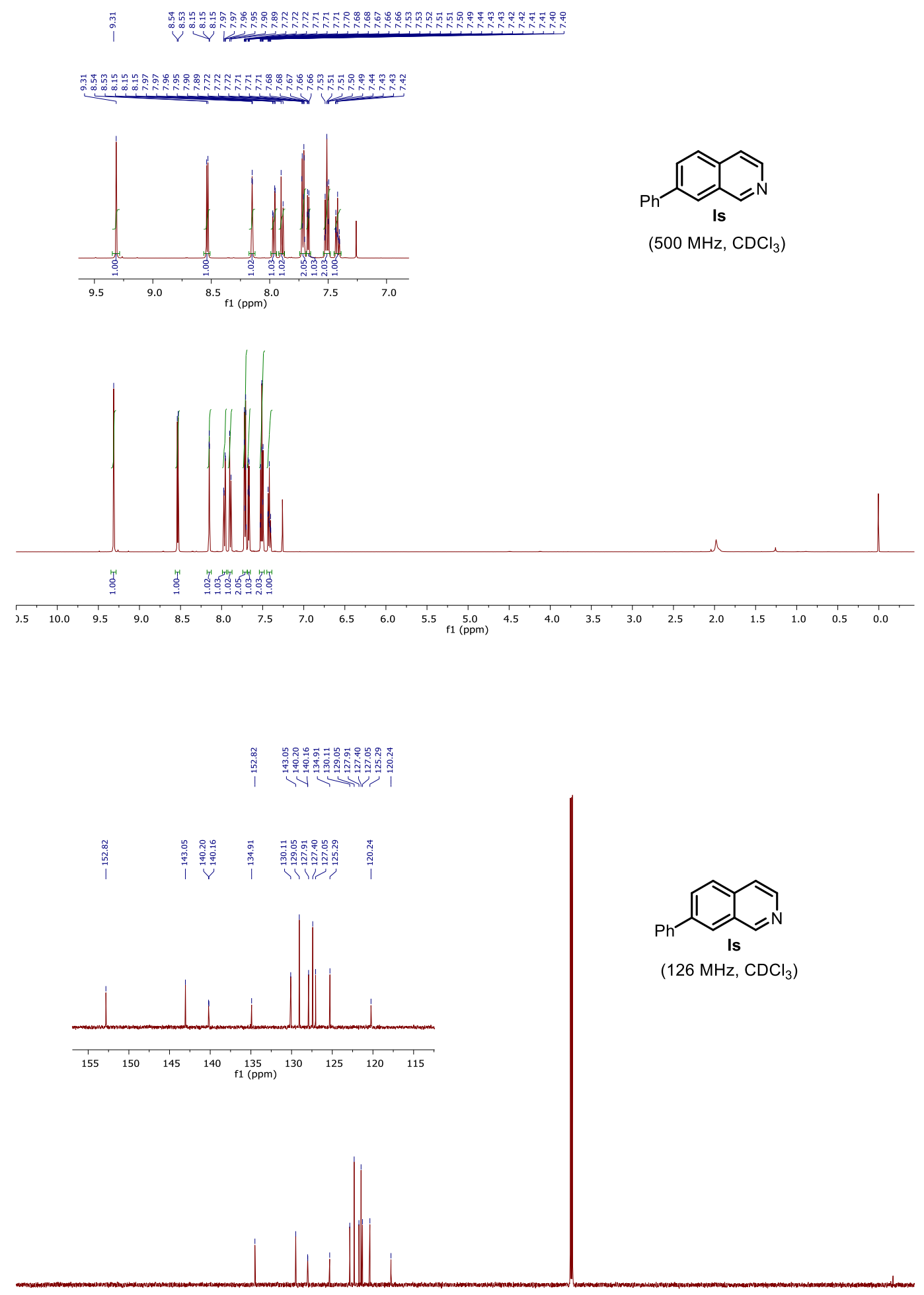

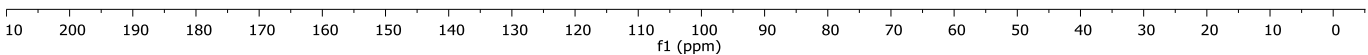



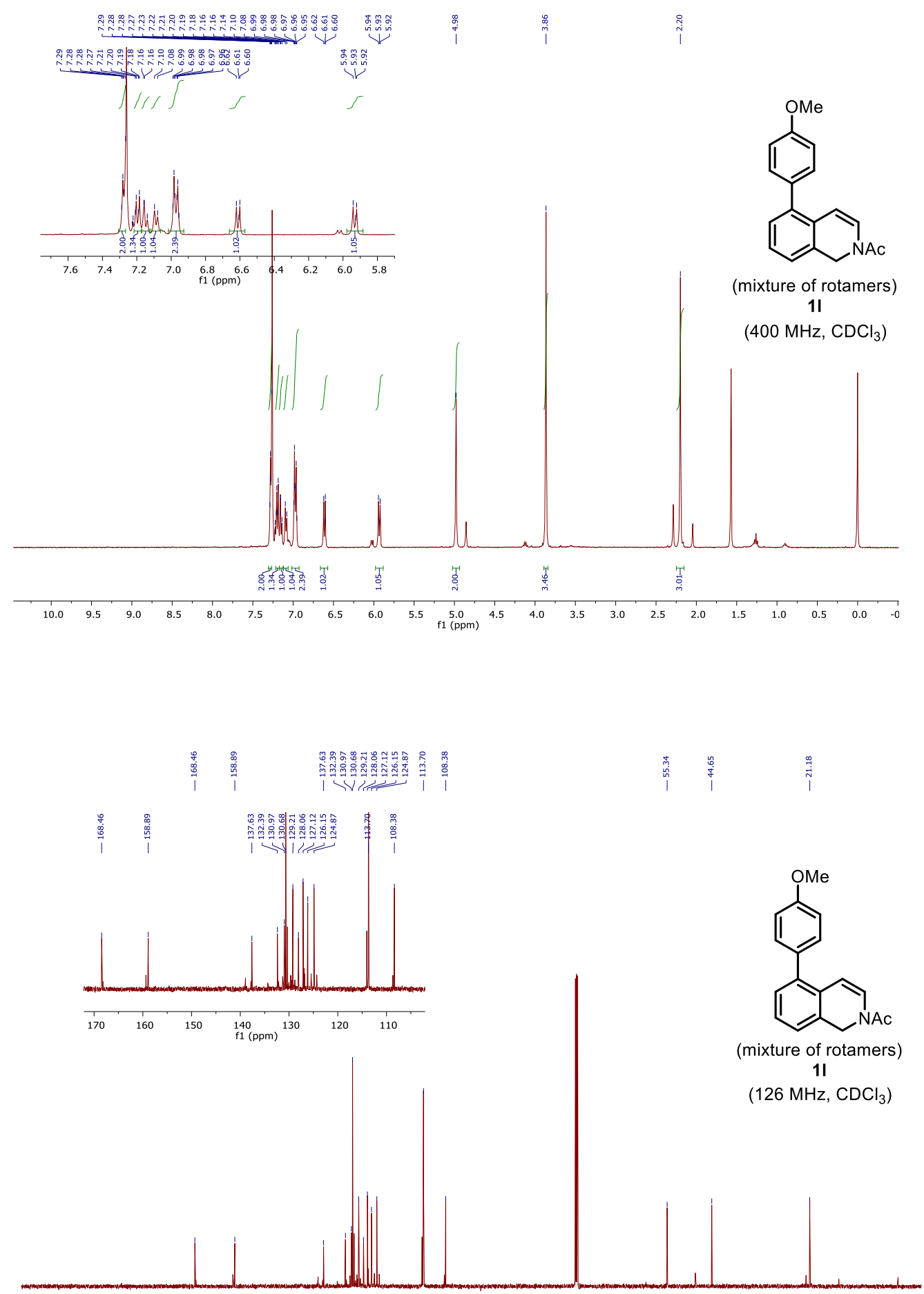

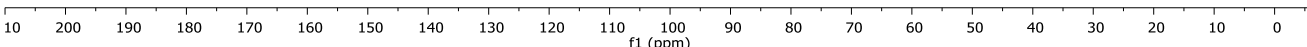



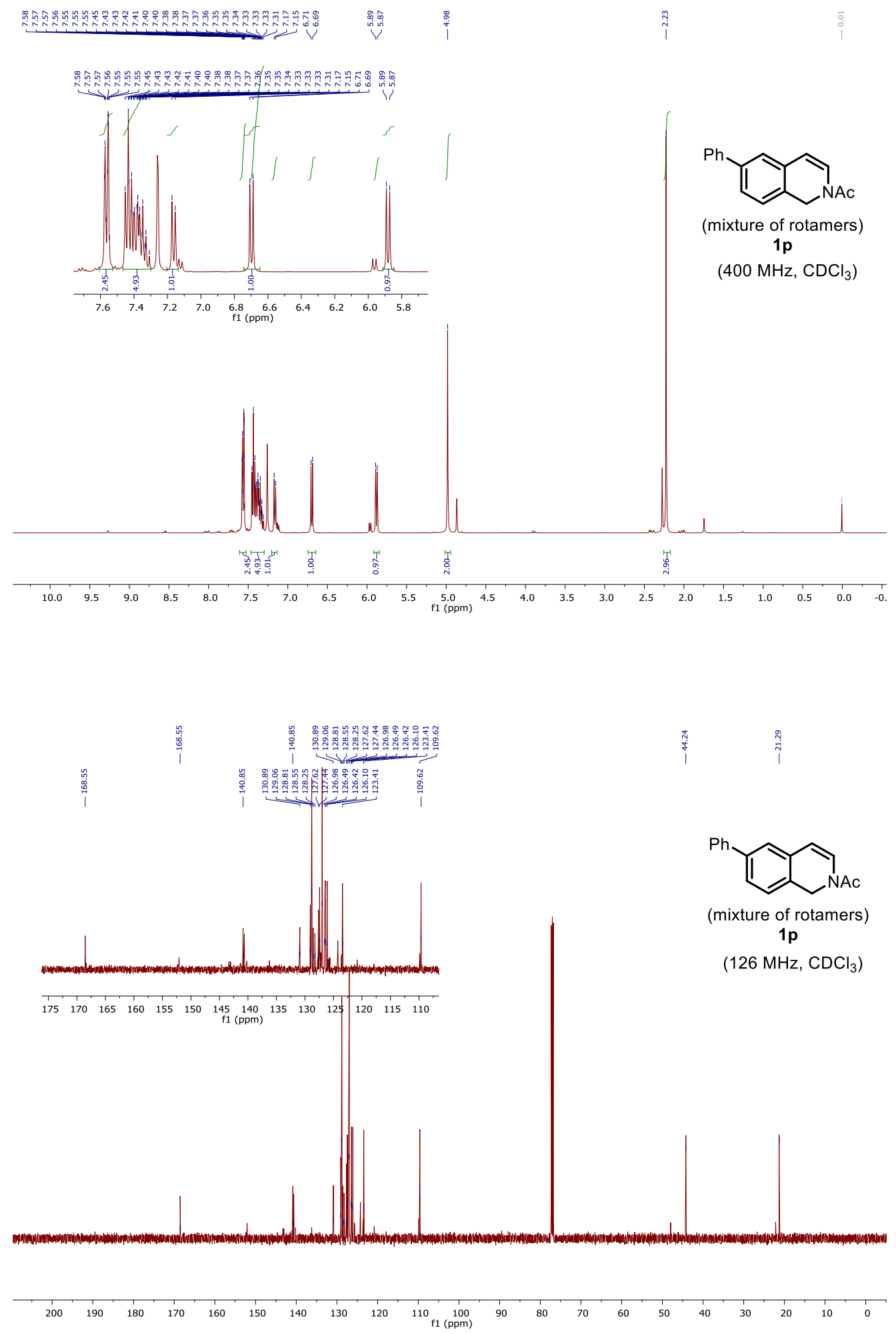

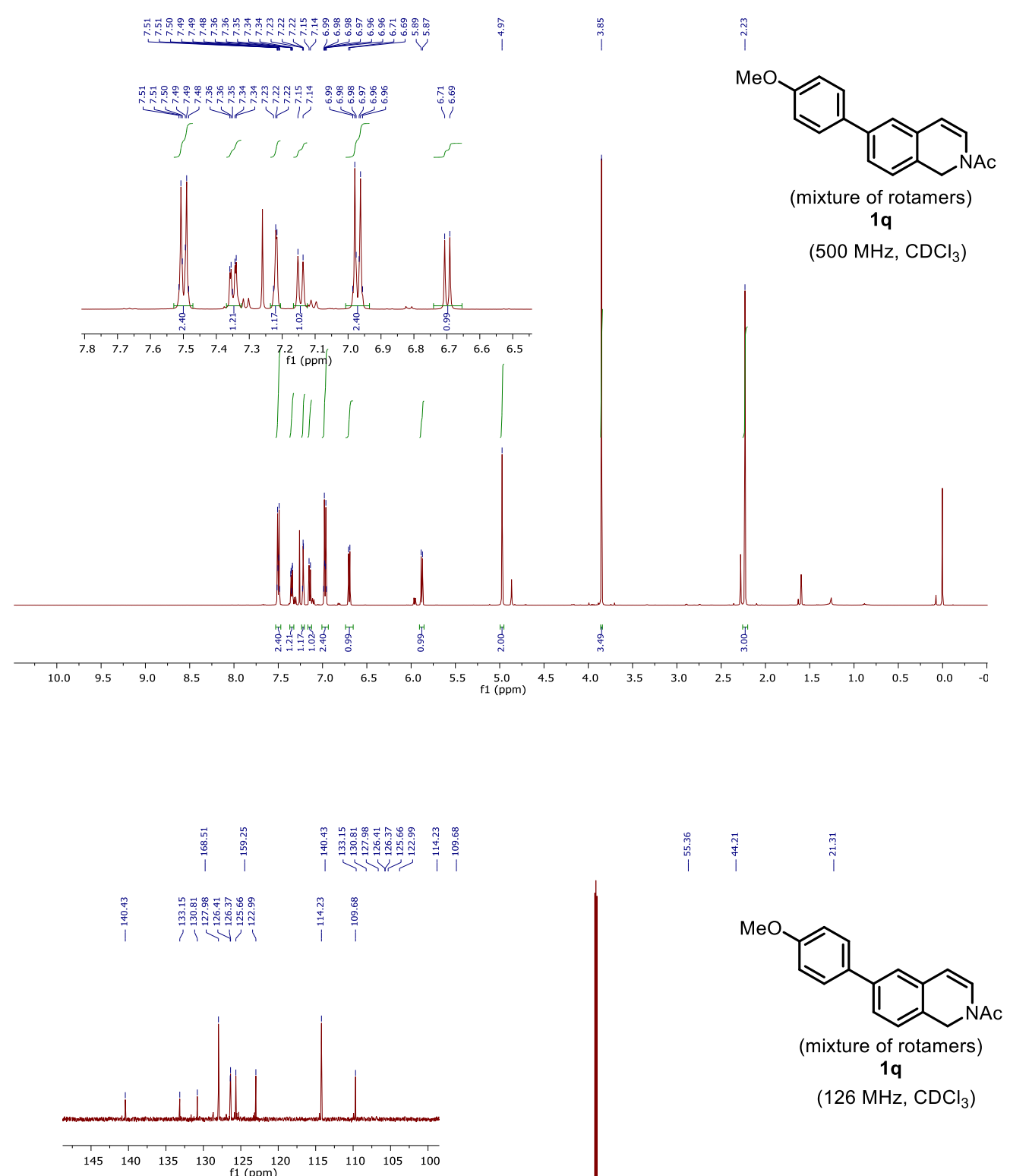

(mixture of rotamers)

19

(126 $\mathrm{MHz} \mathrm{CDCl}_{3}$ )
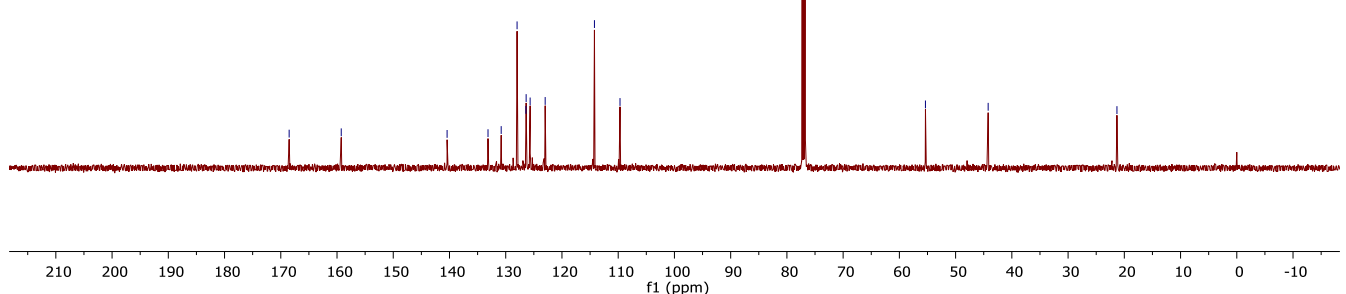


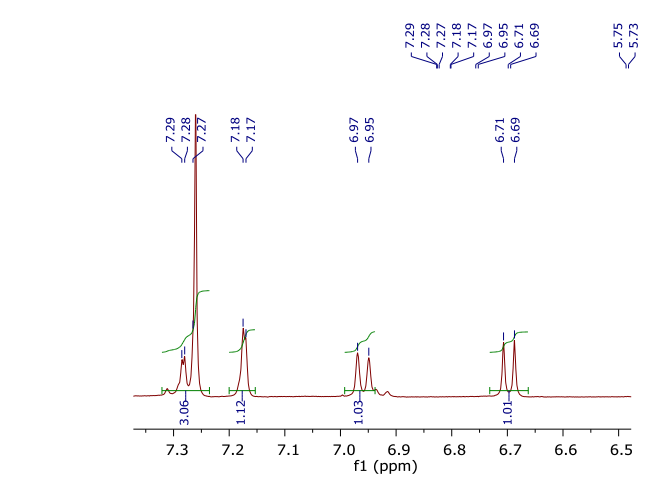

$\underset{\sim}{*}$
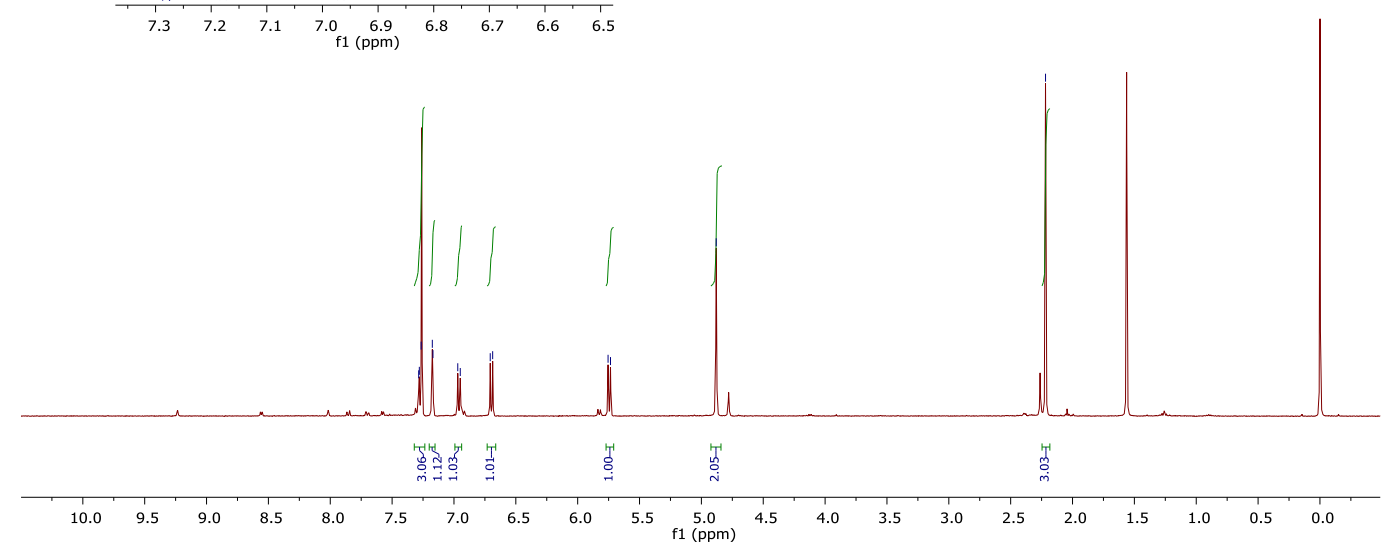

(mixture of rotamers)

$\left(500 \mathrm{MHz}, \mathrm{CDCl}_{3}\right.$ )
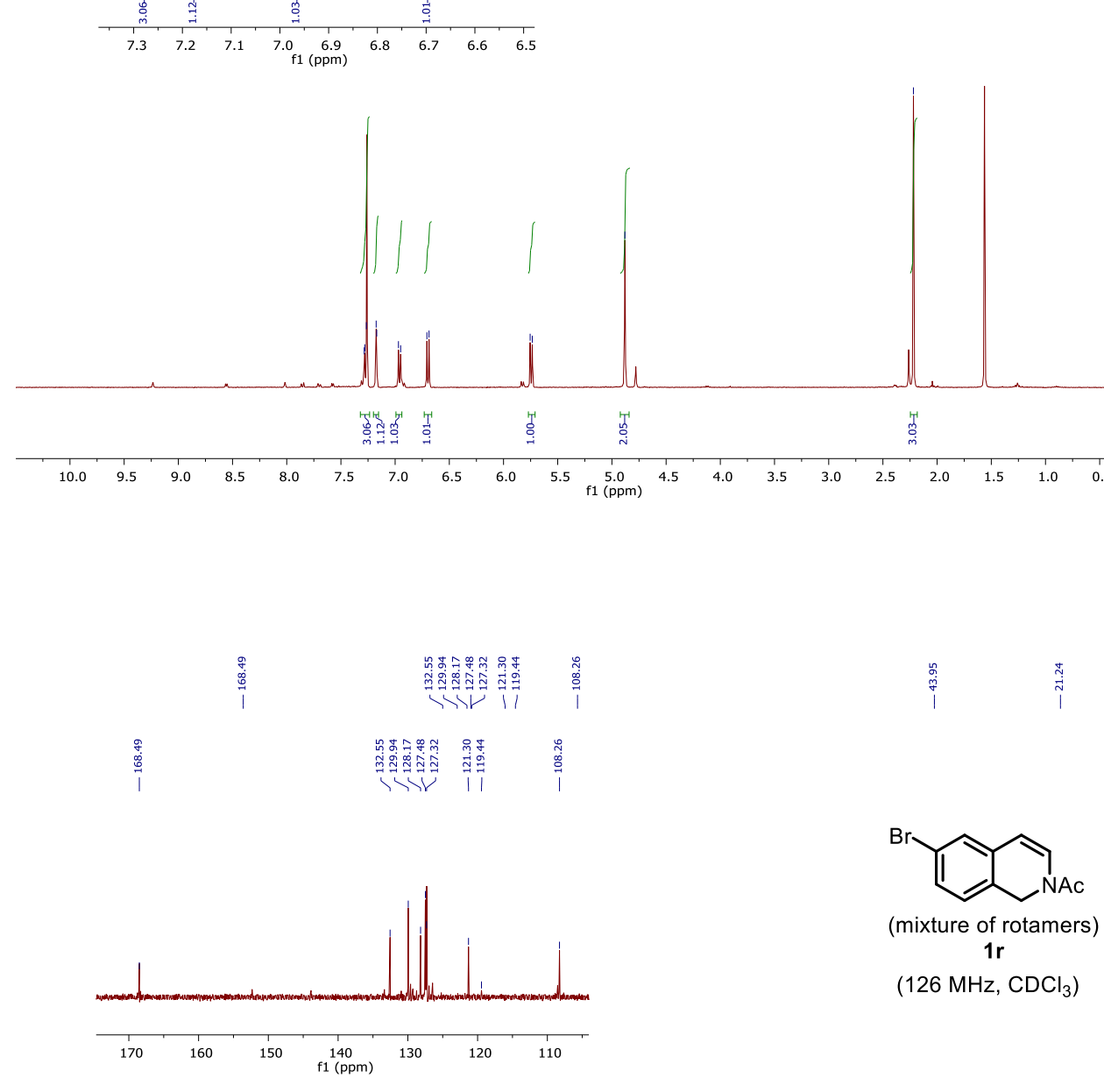

(mixture of rotamers)

$1 \mathrm{r}$

(126 MHz, $\mathrm{CDCl}_{3}$ )

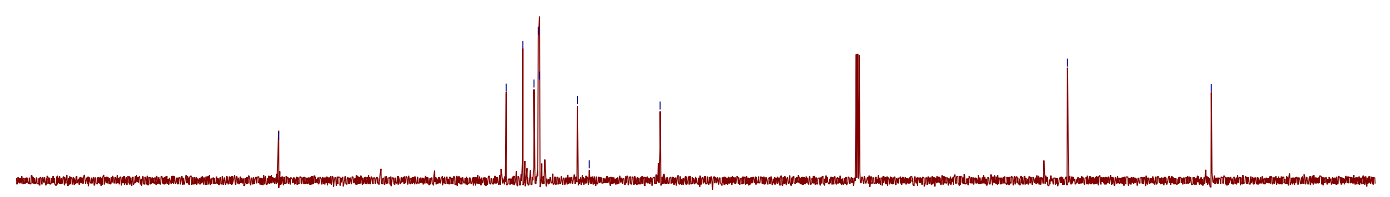



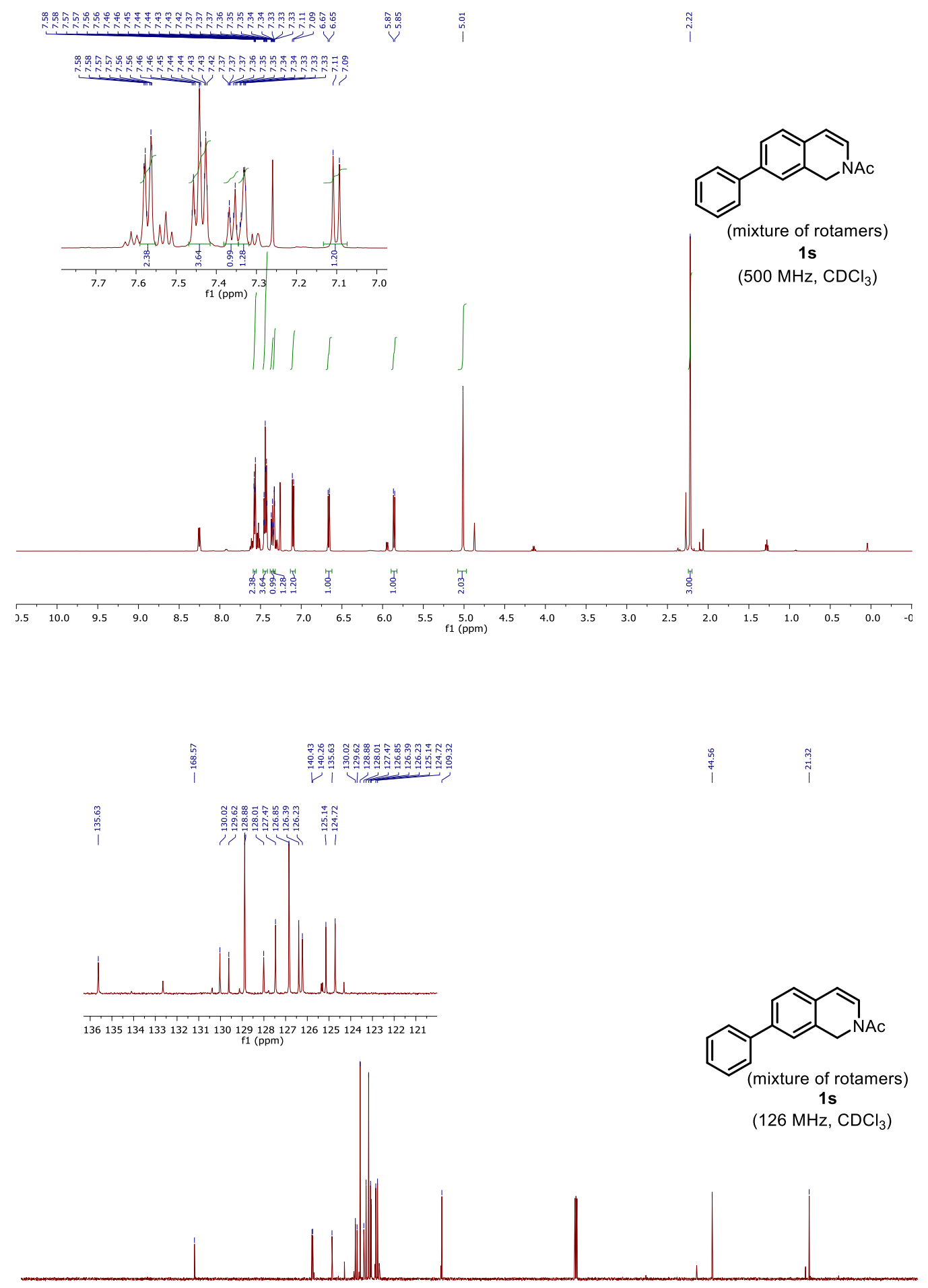

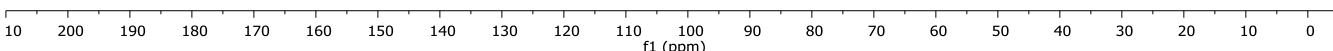




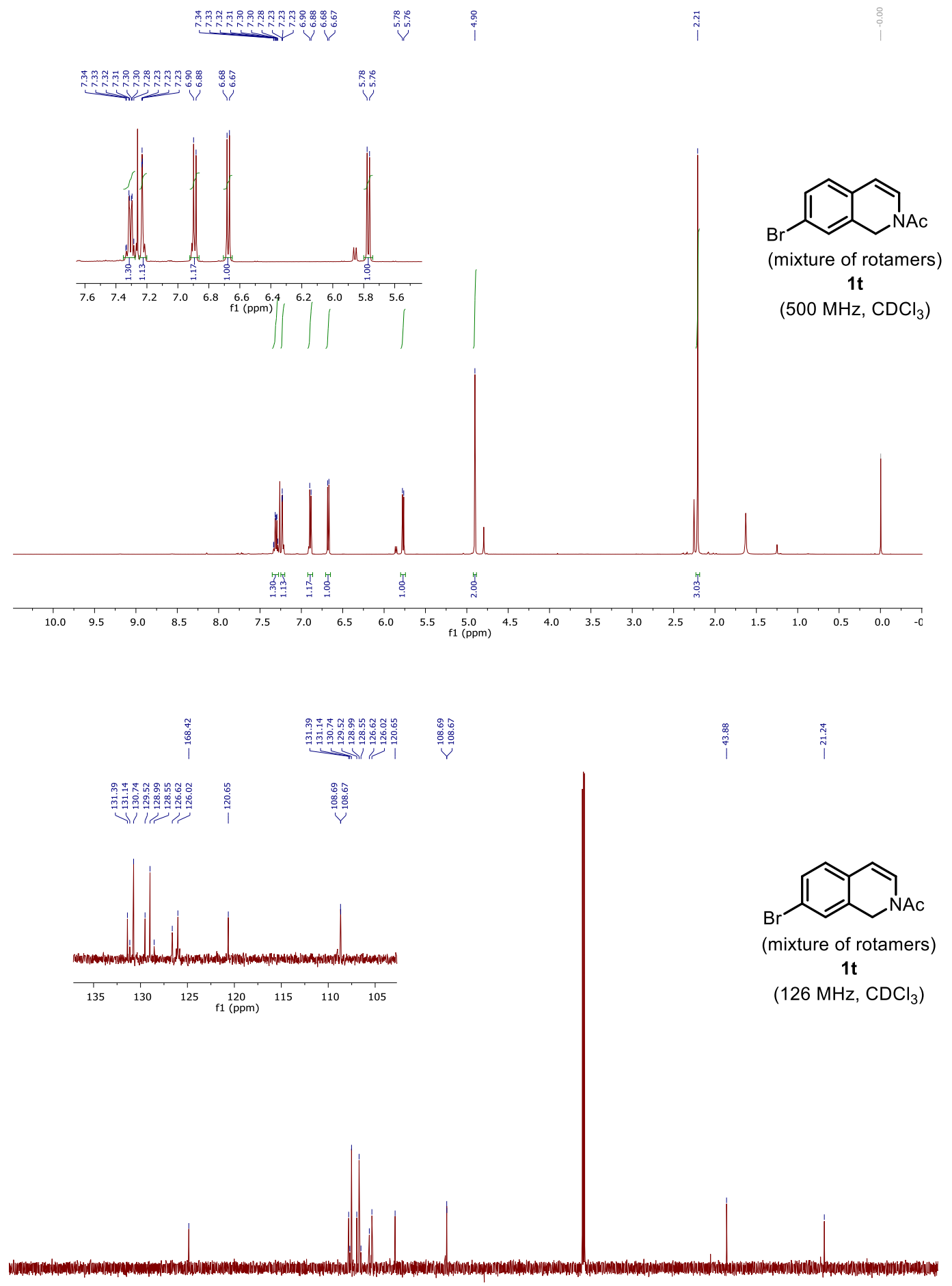

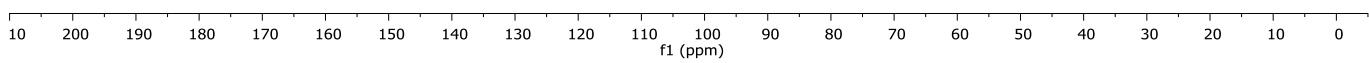




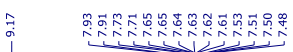
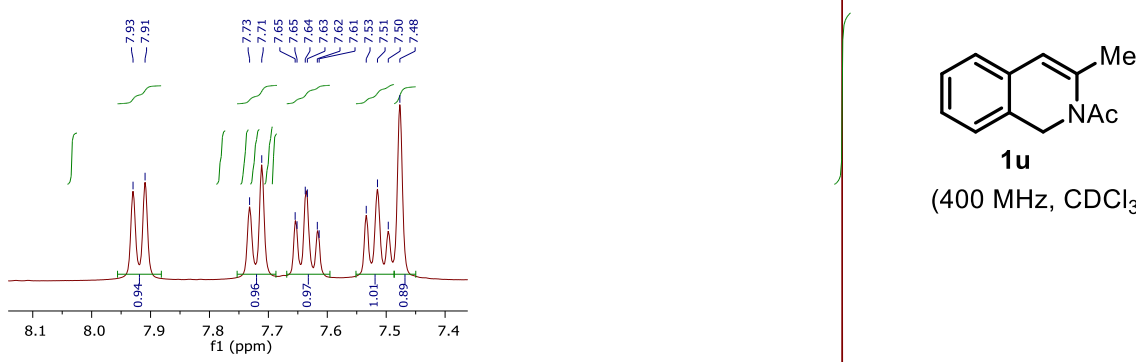

$1 \mathrm{u}$

(400 MHz, $\mathrm{CDCl}_{3}$ )

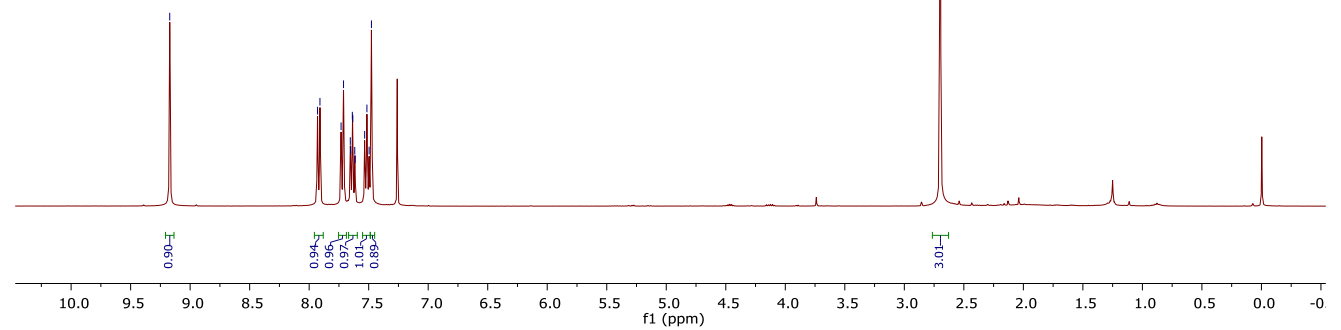

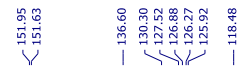
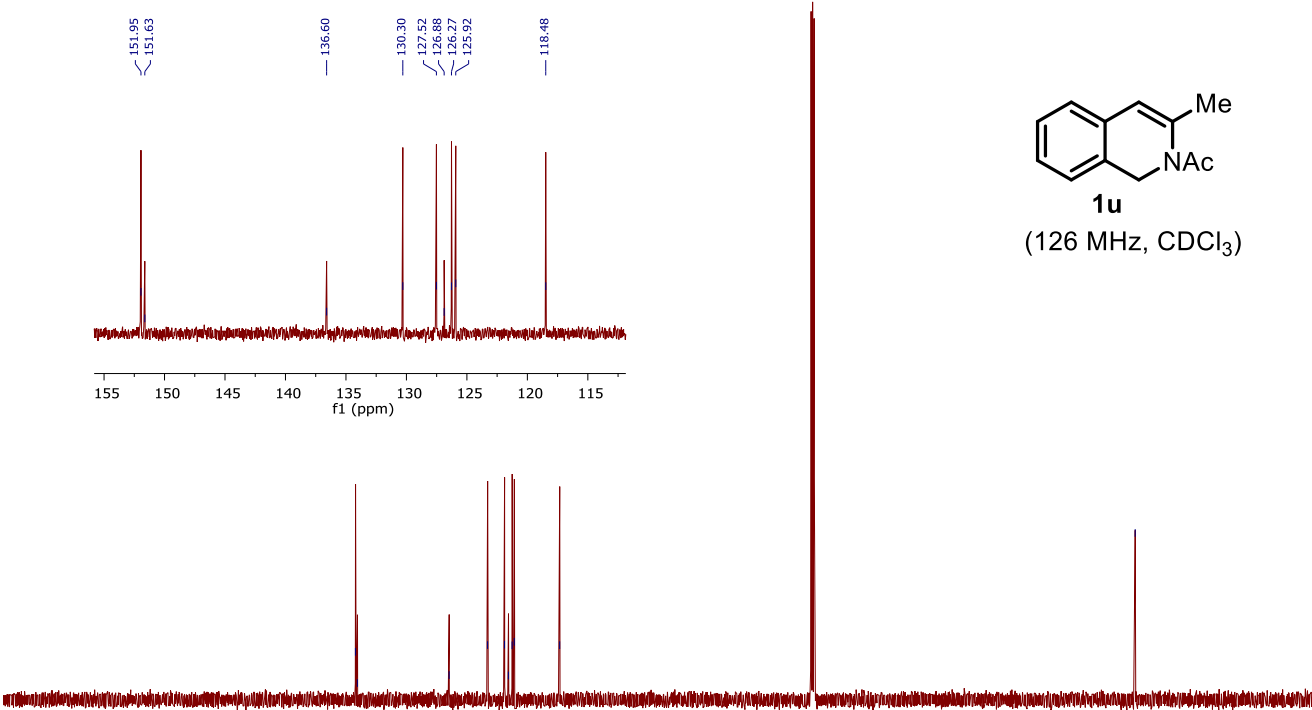

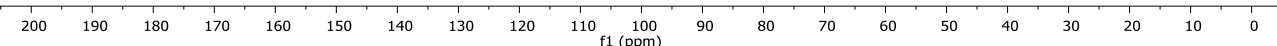




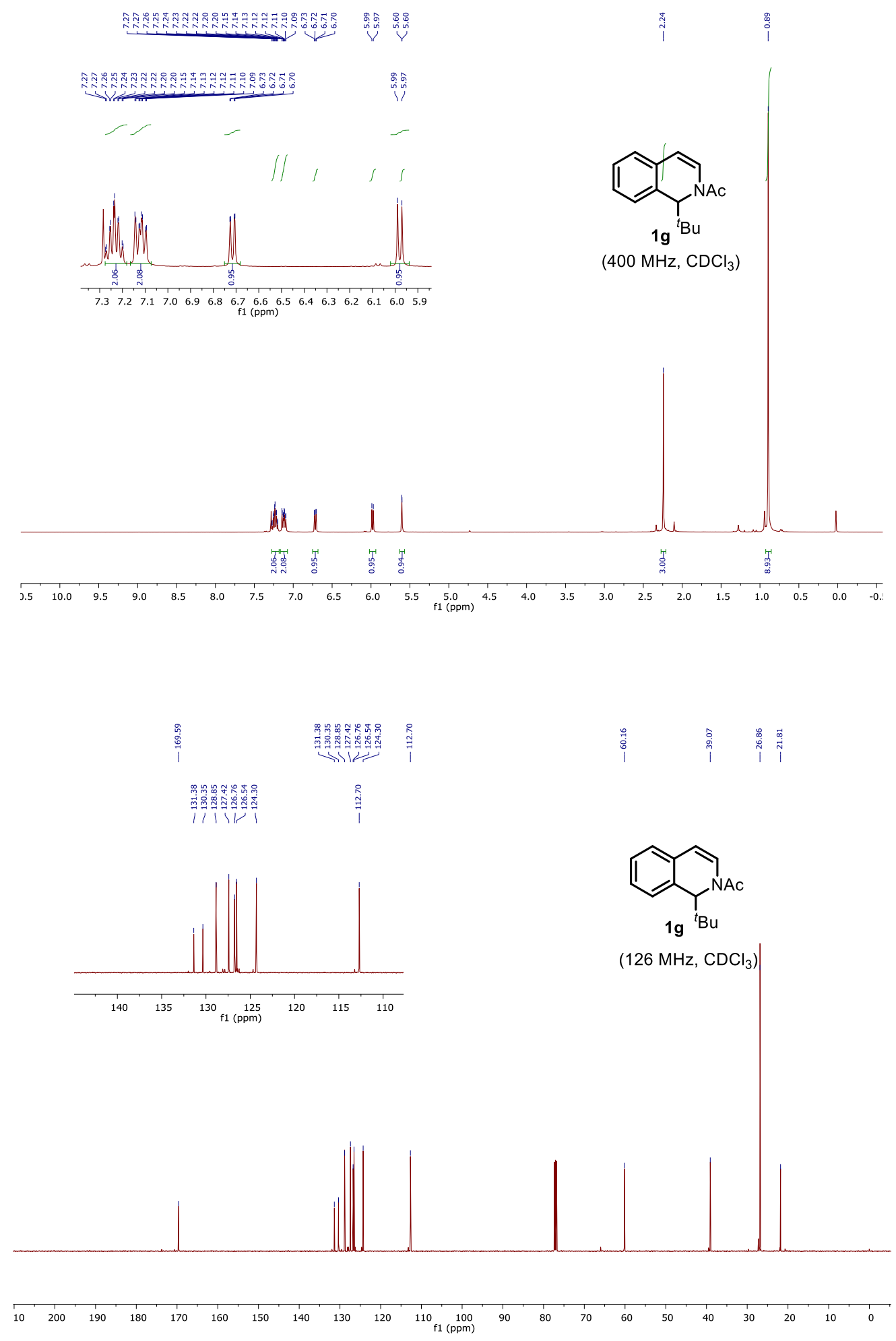

Page S51 of S103 

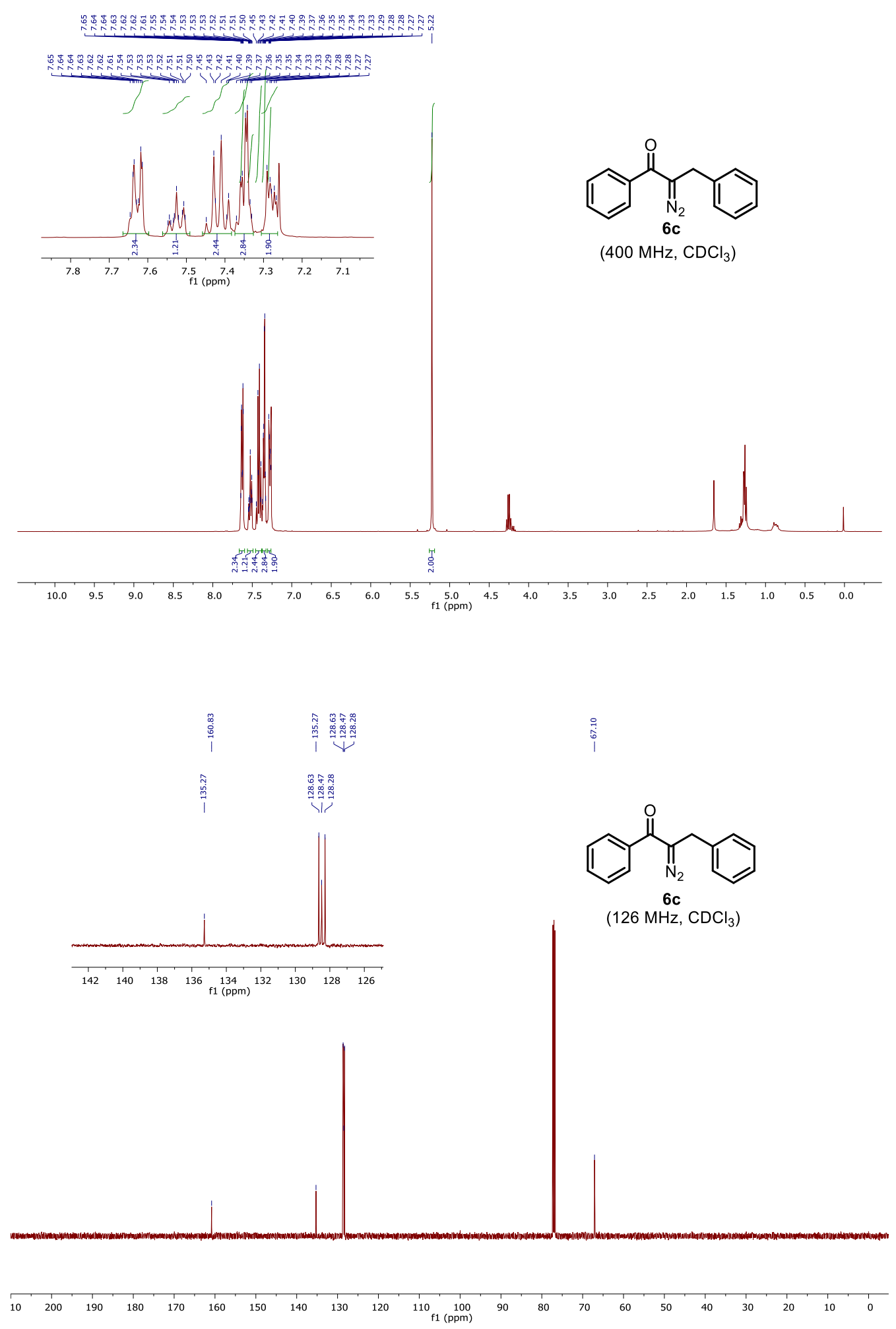


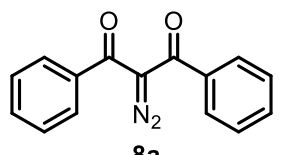

$\left(500 \mathrm{MHz}, \mathrm{CDCl}_{3}\right)$

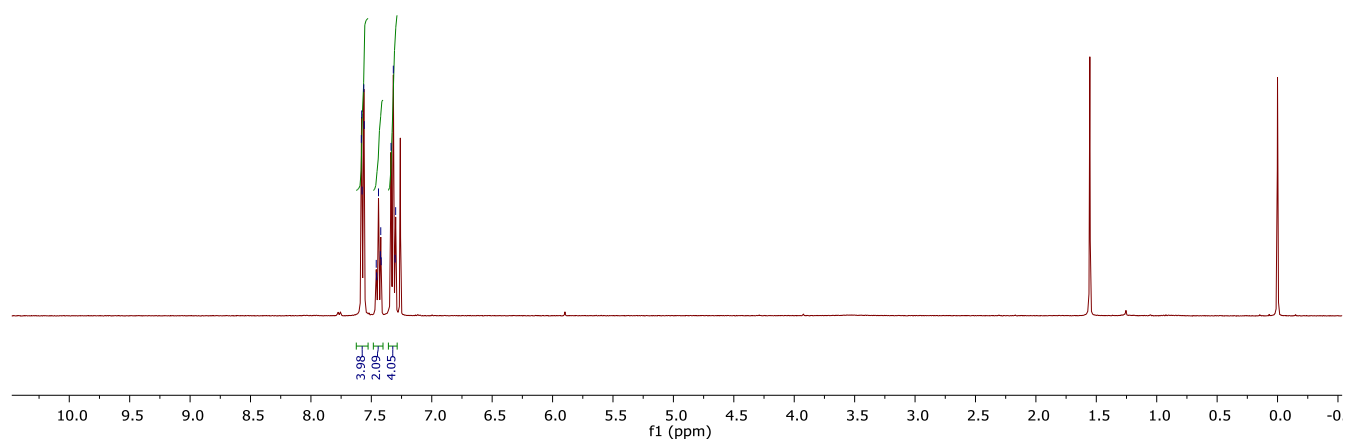

\begin{tabular}{|c|c|}
\hline 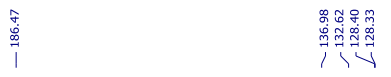 & 每 \\
\hline
\end{tabular}

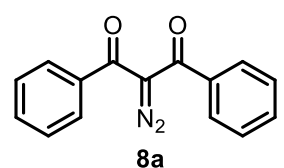

$\left(126 \mathrm{MHz}, \mathrm{CDCl}_{3}\right)$

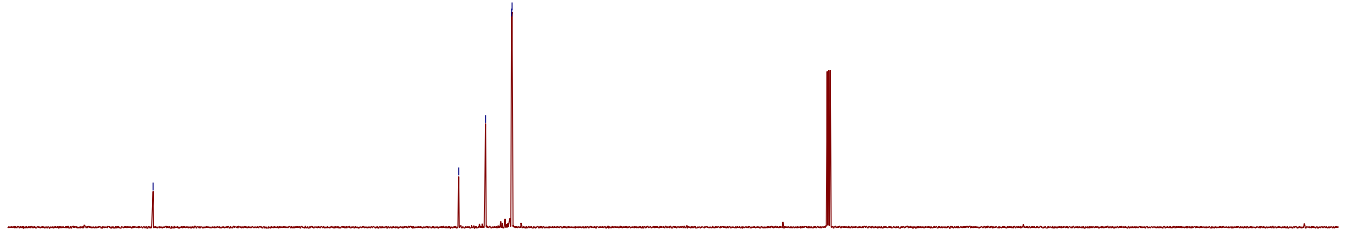

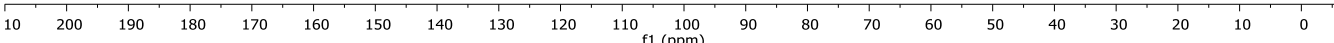




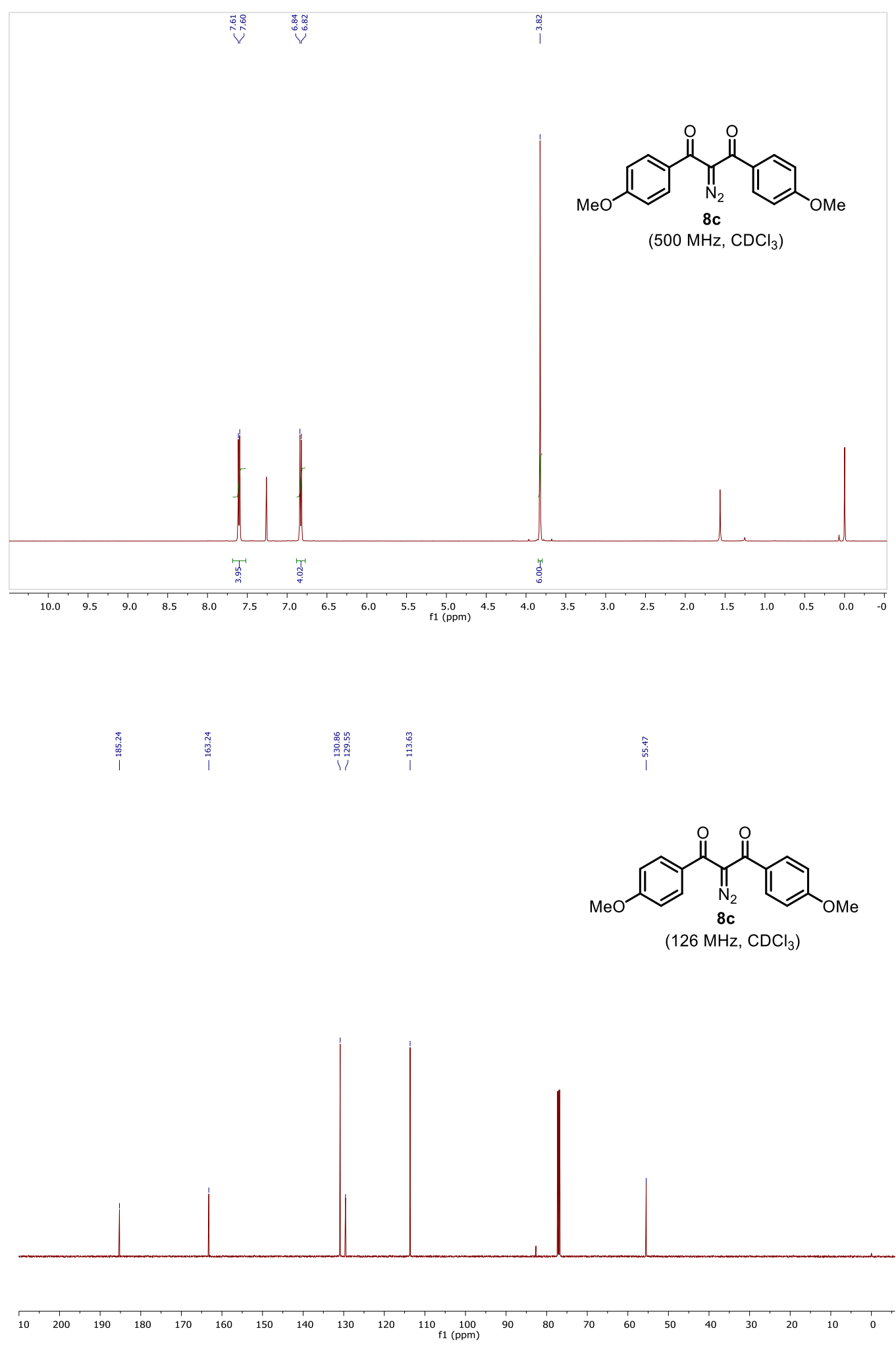



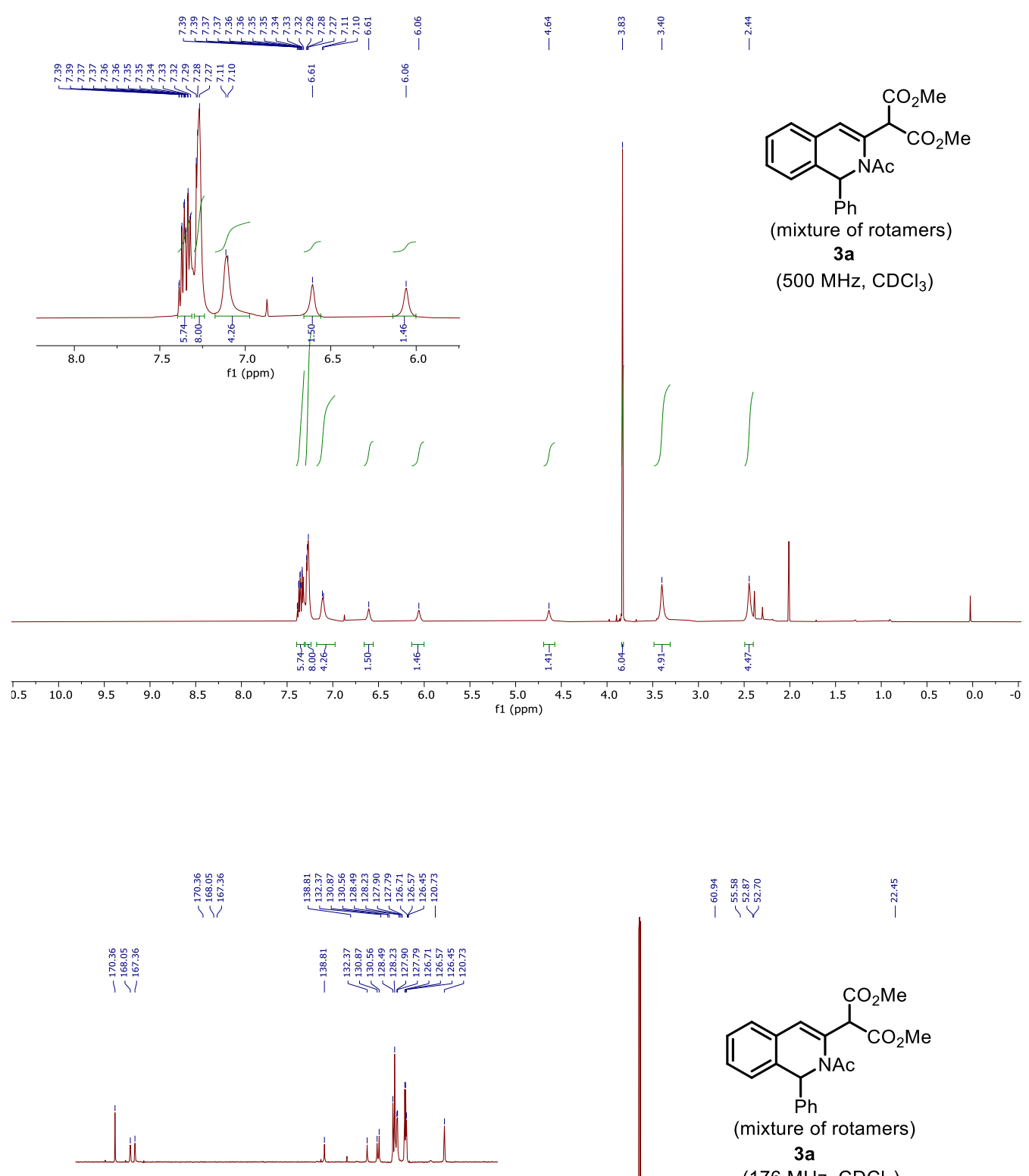

$\begin{array}{lllllllllllllllll}175 & 170 & 165 & 160 & 155 & 150 & 145 & 140 & 135 & 130 & 125 & 120 & 115\end{array}$
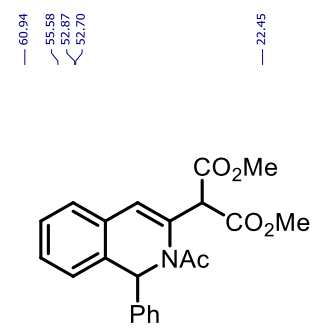

(mixture of rotamers)

$3 a$

$\left.\left(176 \mathrm{MHz}^{\mathrm{CDCl}}\right)_{3}\right)$

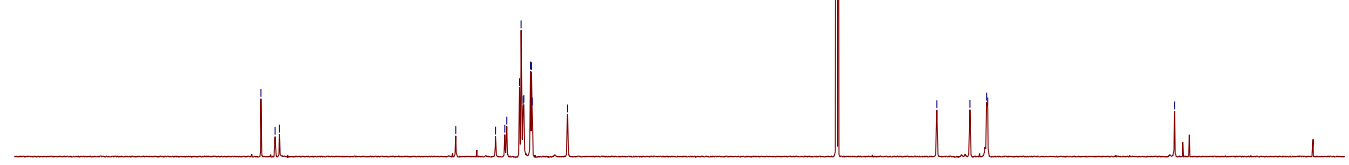

$\begin{array}{llllllllllllllllllllll}1 & 200 & 190 & 180 & 170 & 160 & 150 & 140 & 130 & 120 & 110 & 100 & 90 & 80 & 70 & 60 & 50 & 40 & 30 & 20 & 10 & 0\end{array}$ 


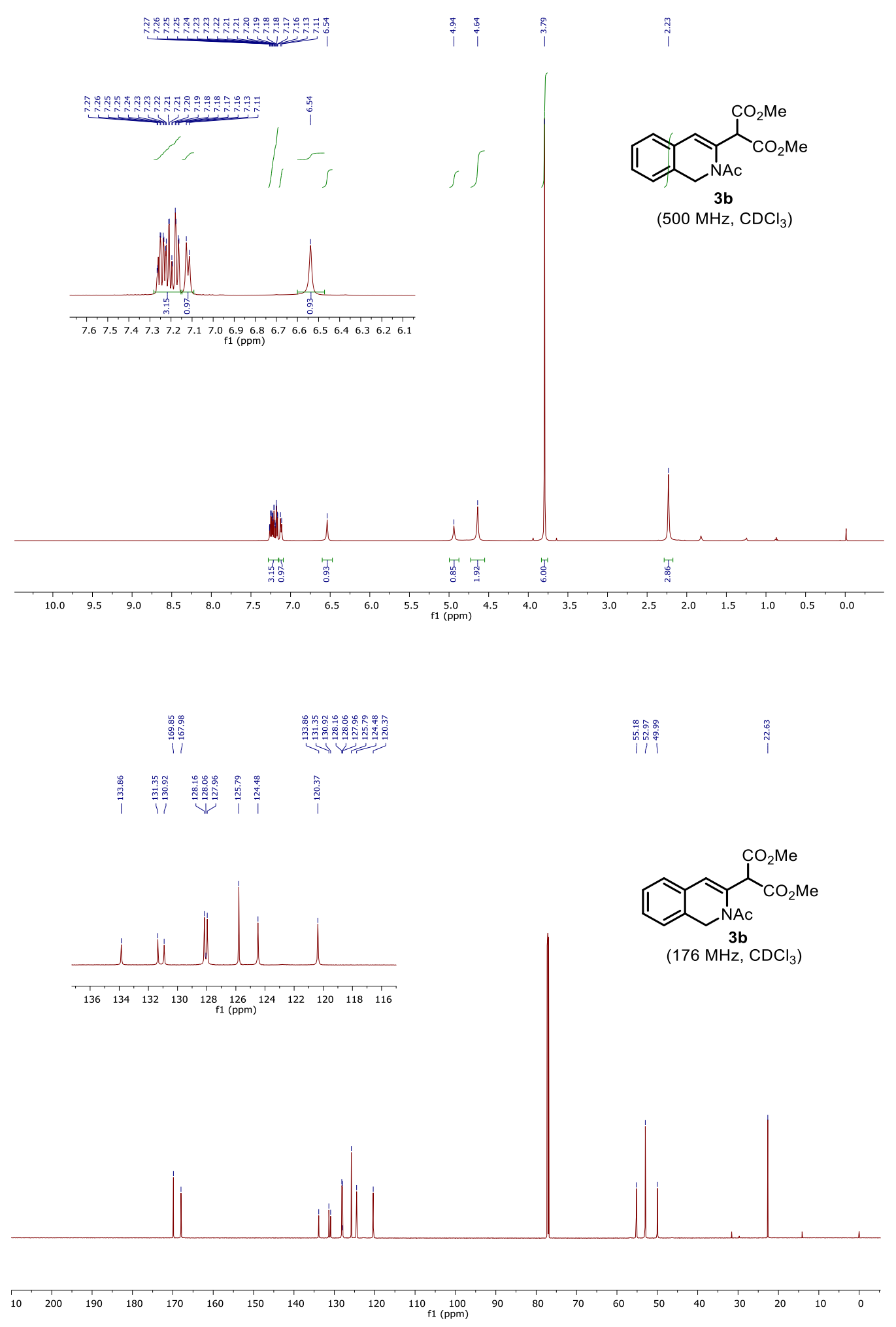

Page S56 of S103 

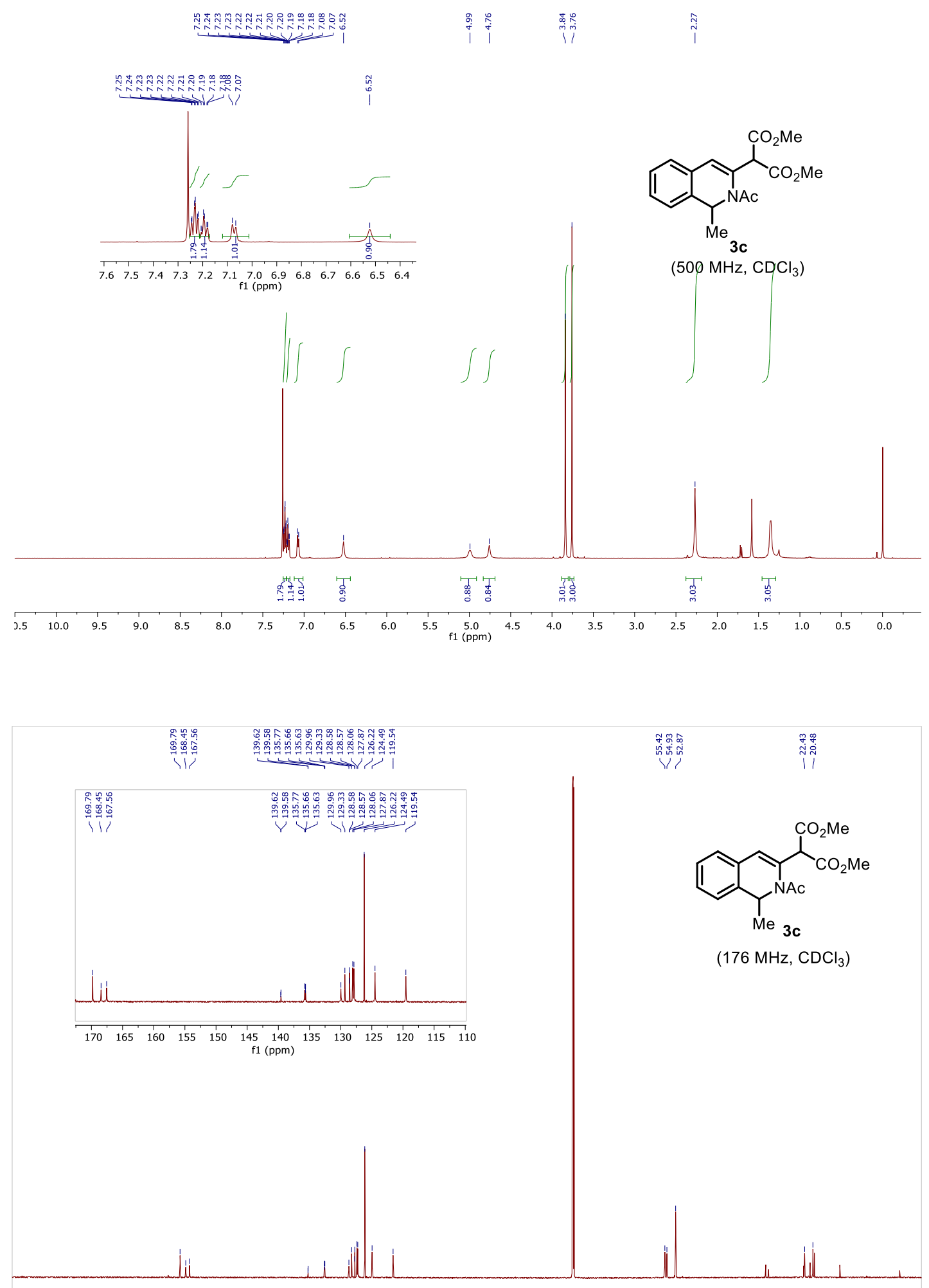

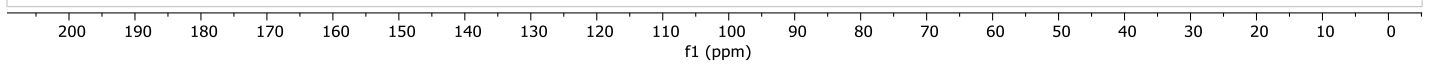




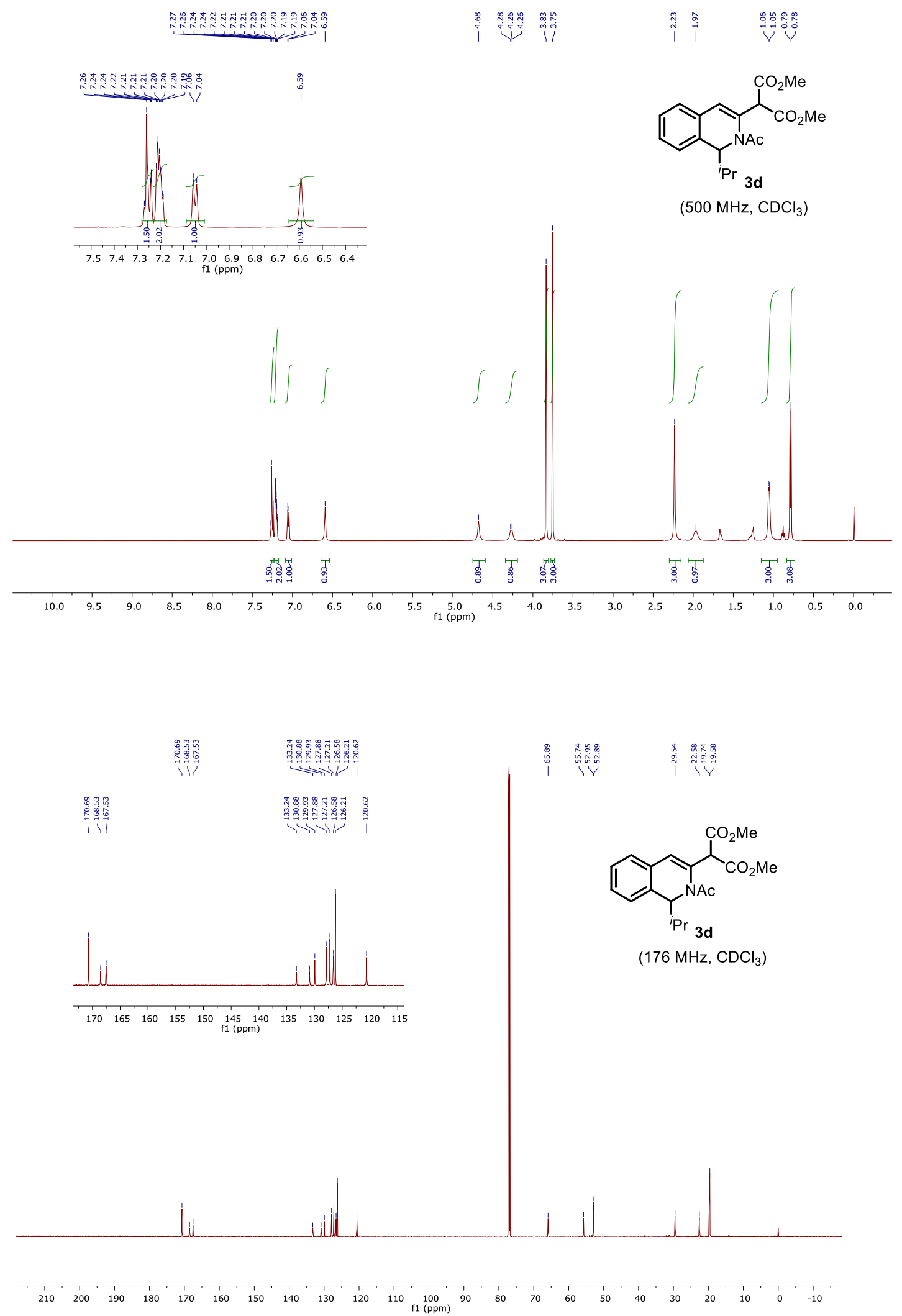

Page S58 of S103 

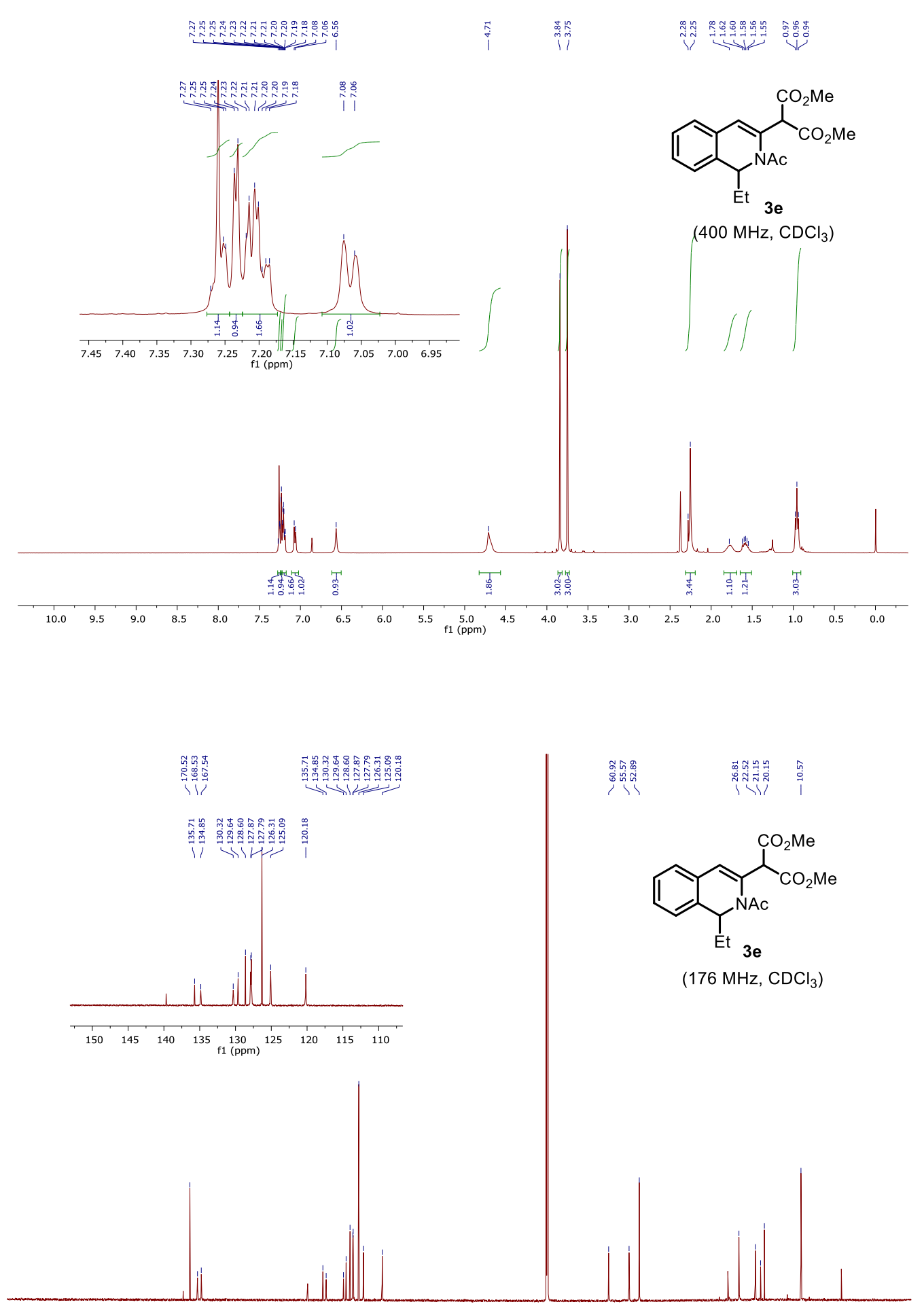

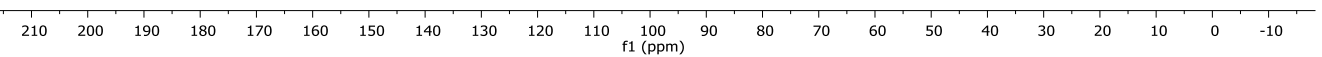



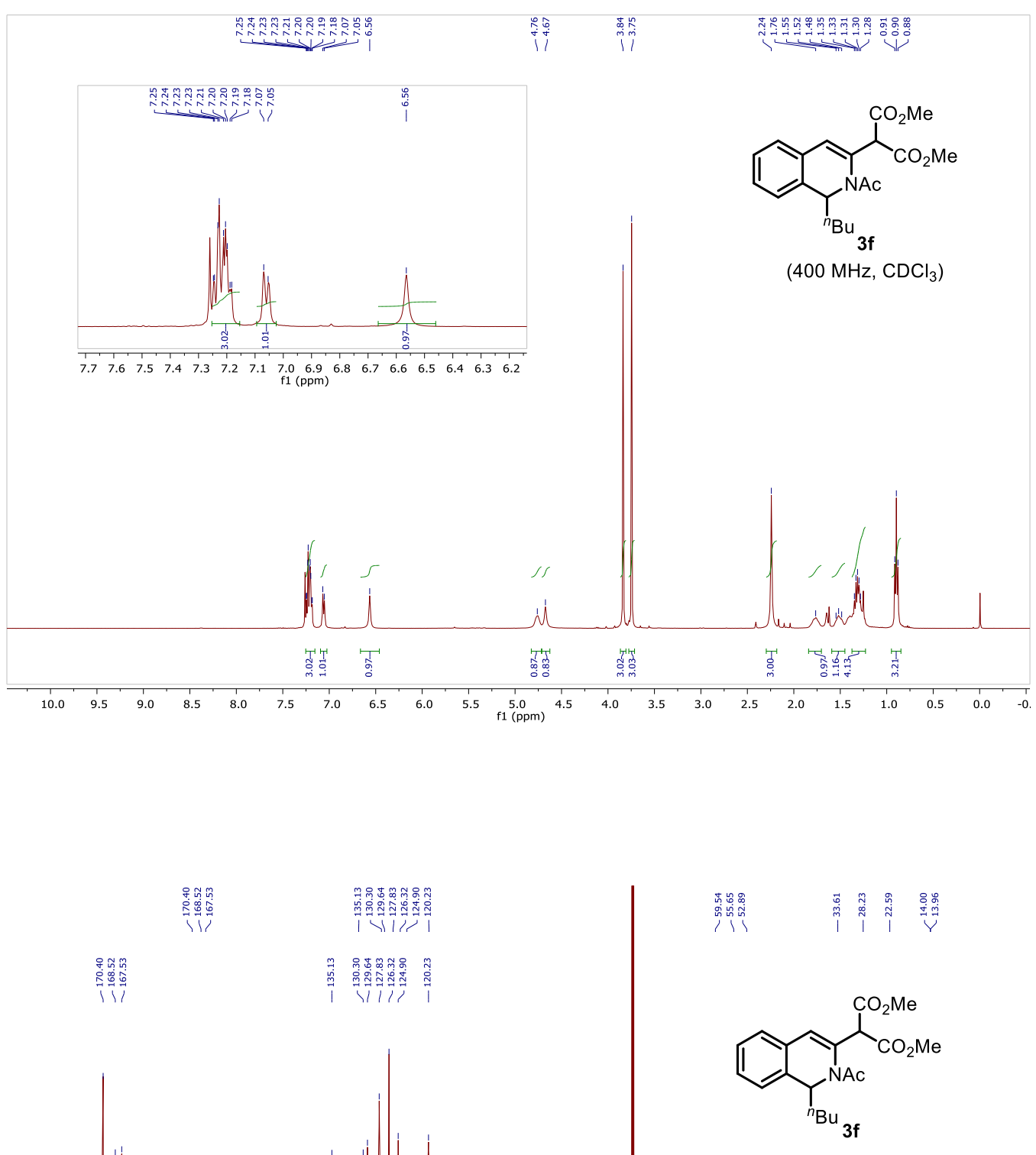

(176 MHz, $\mathrm{CDCl}_{3}$ )
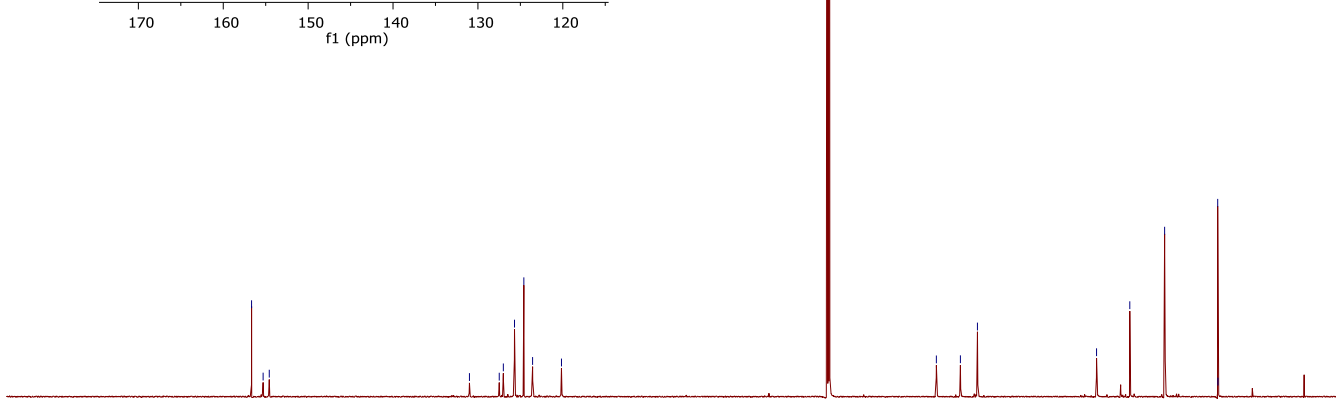

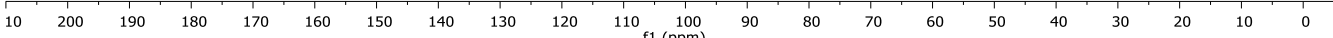




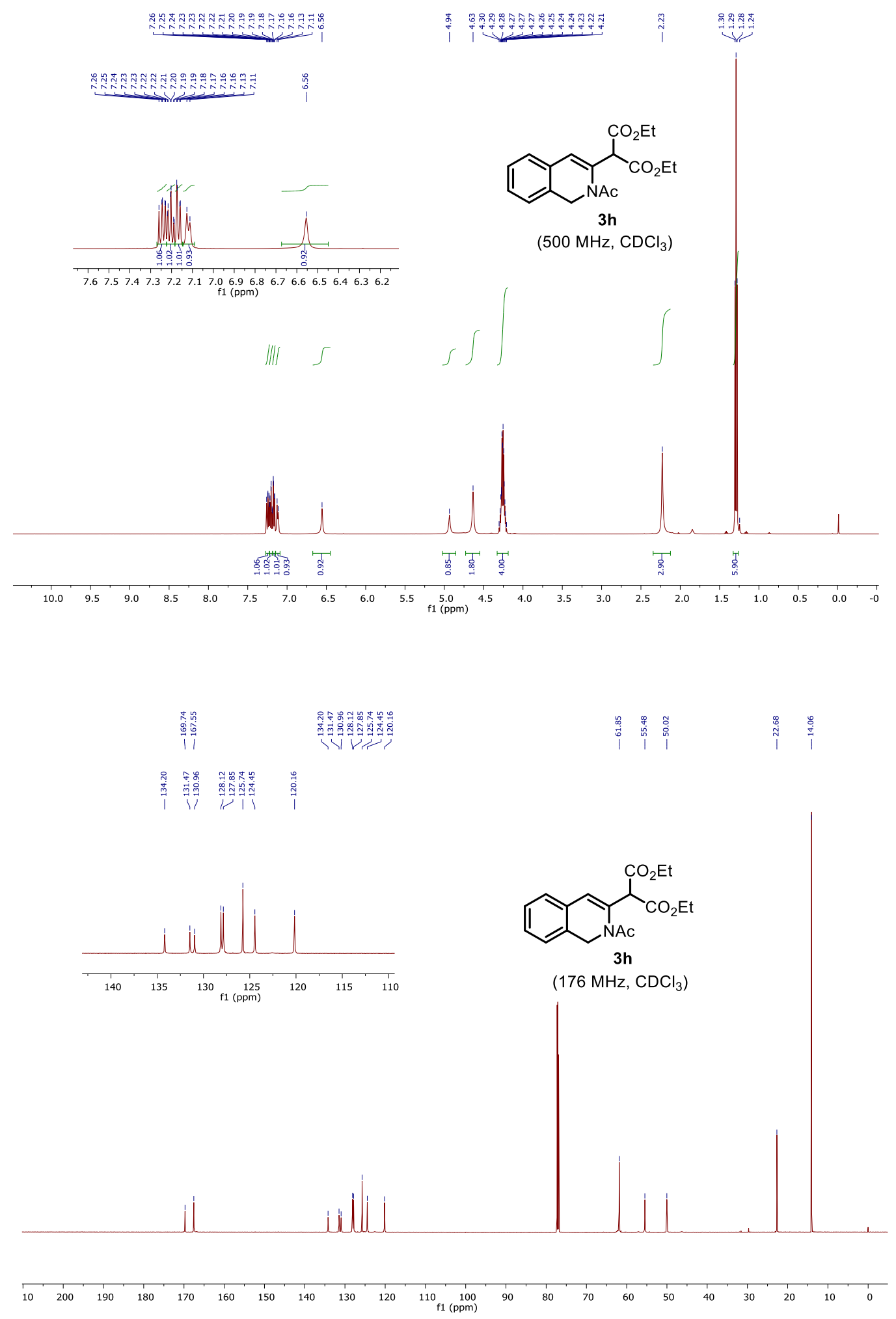

Page S61 of S103 


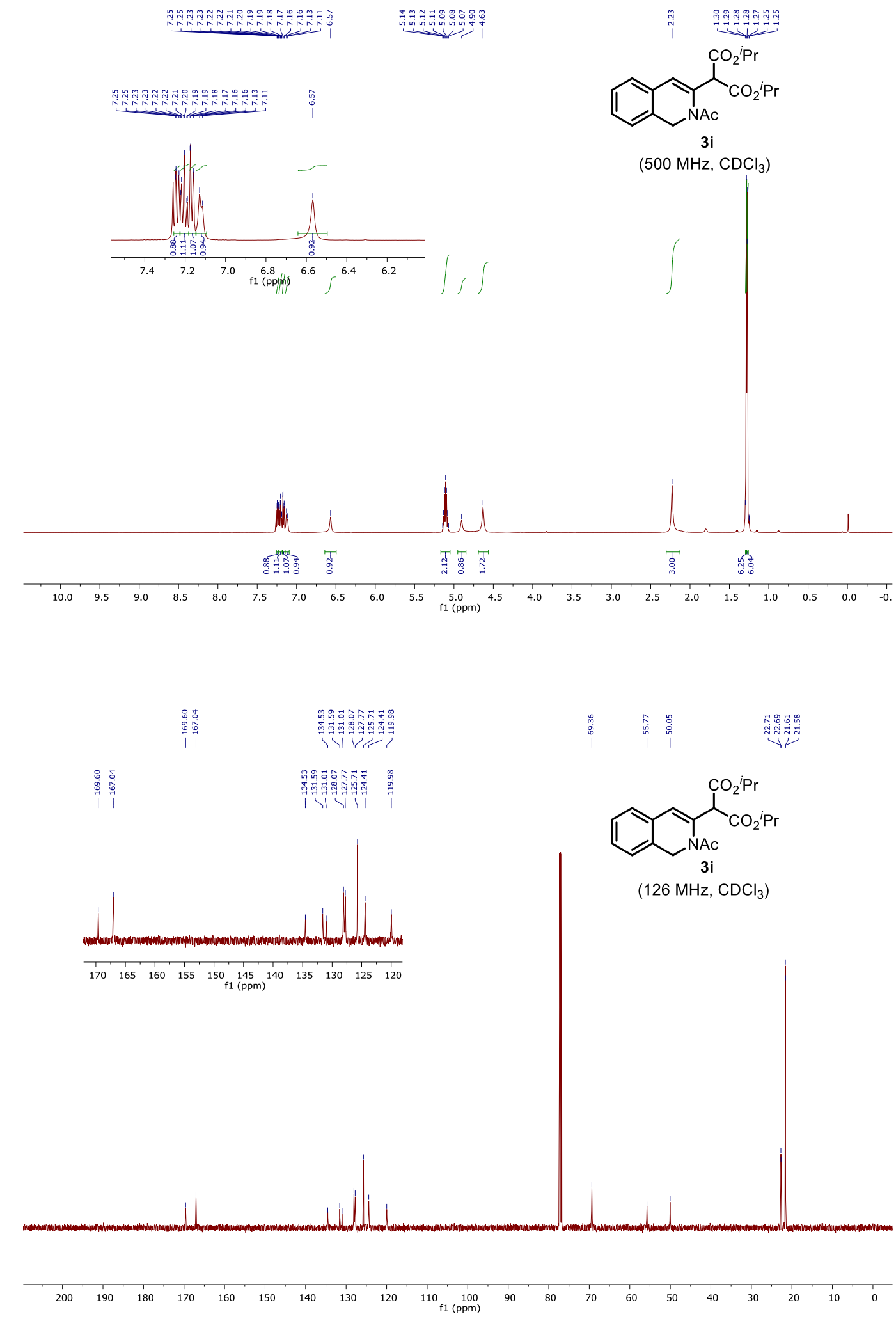

Page S62 of S103 

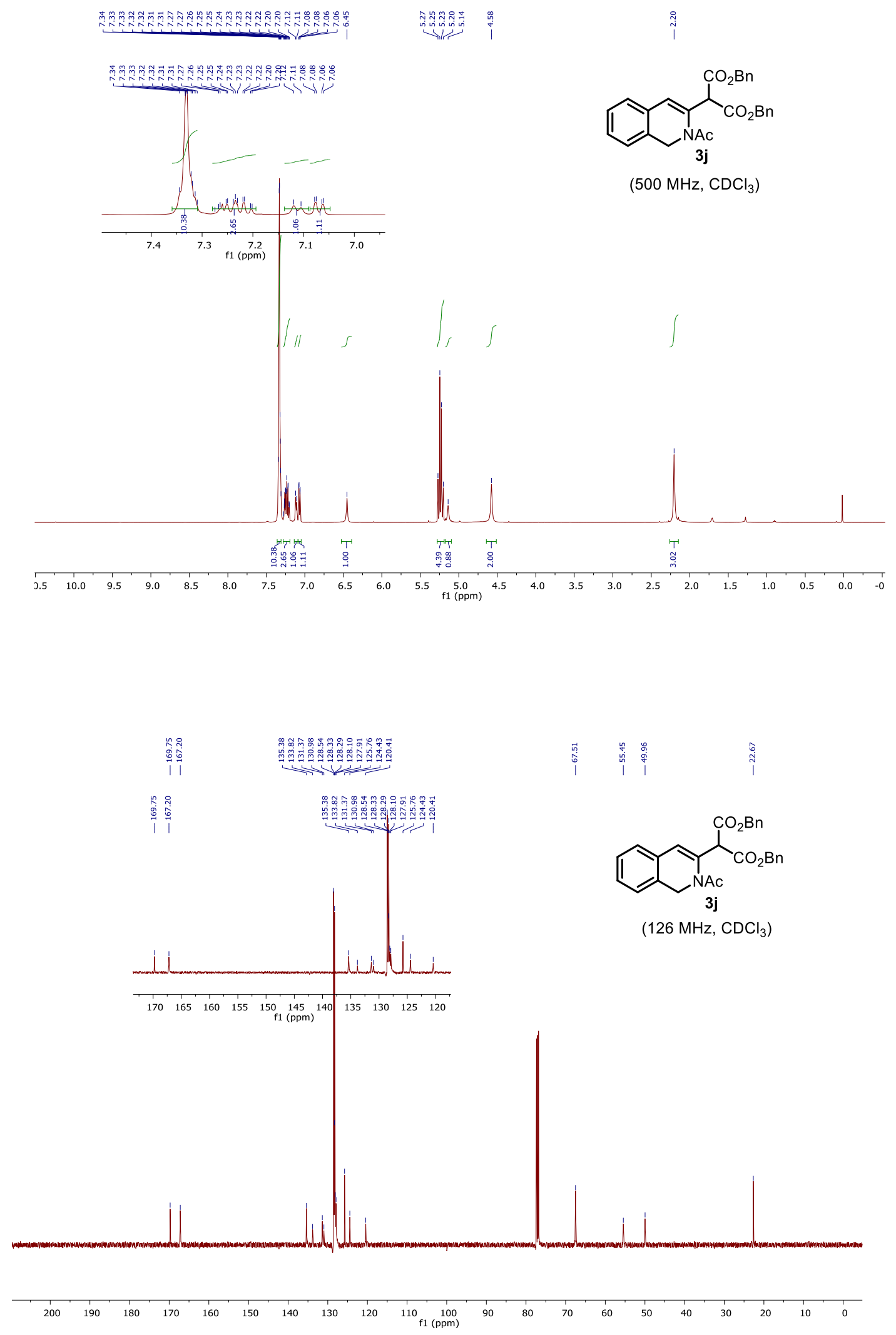

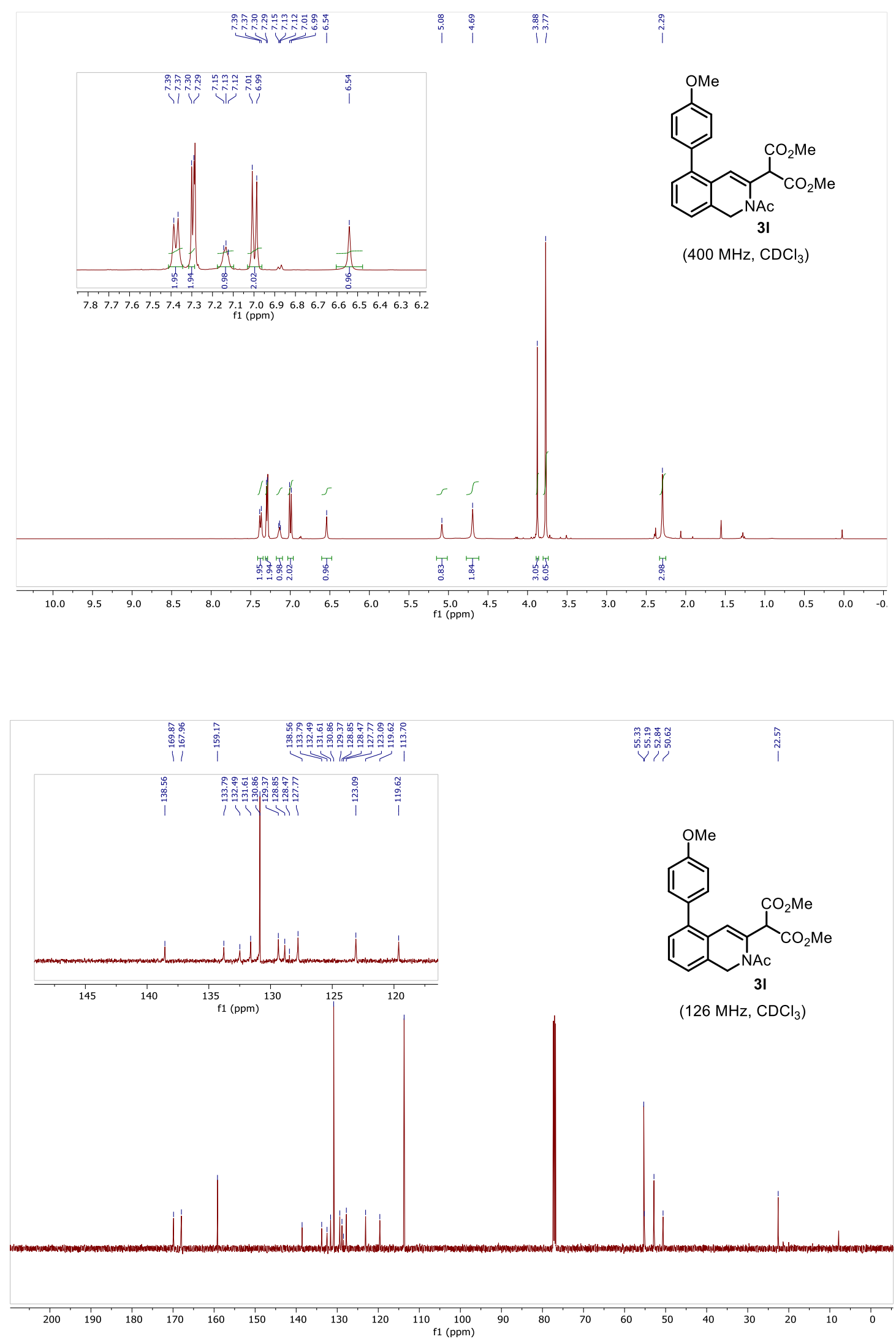


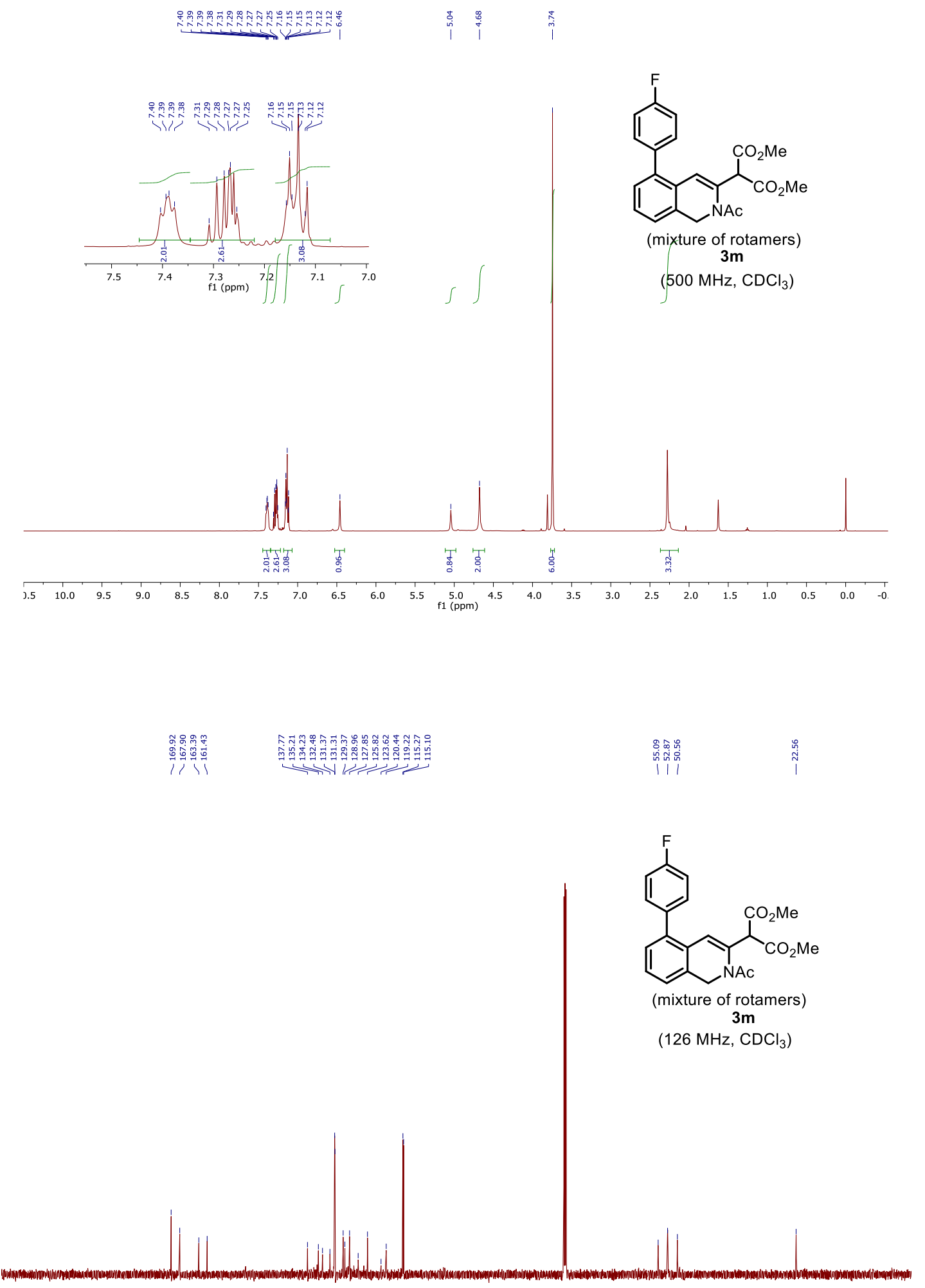

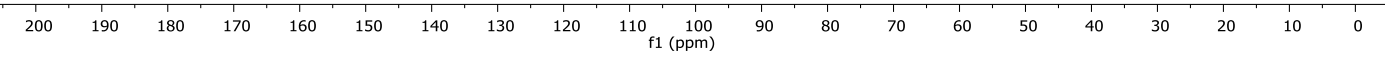



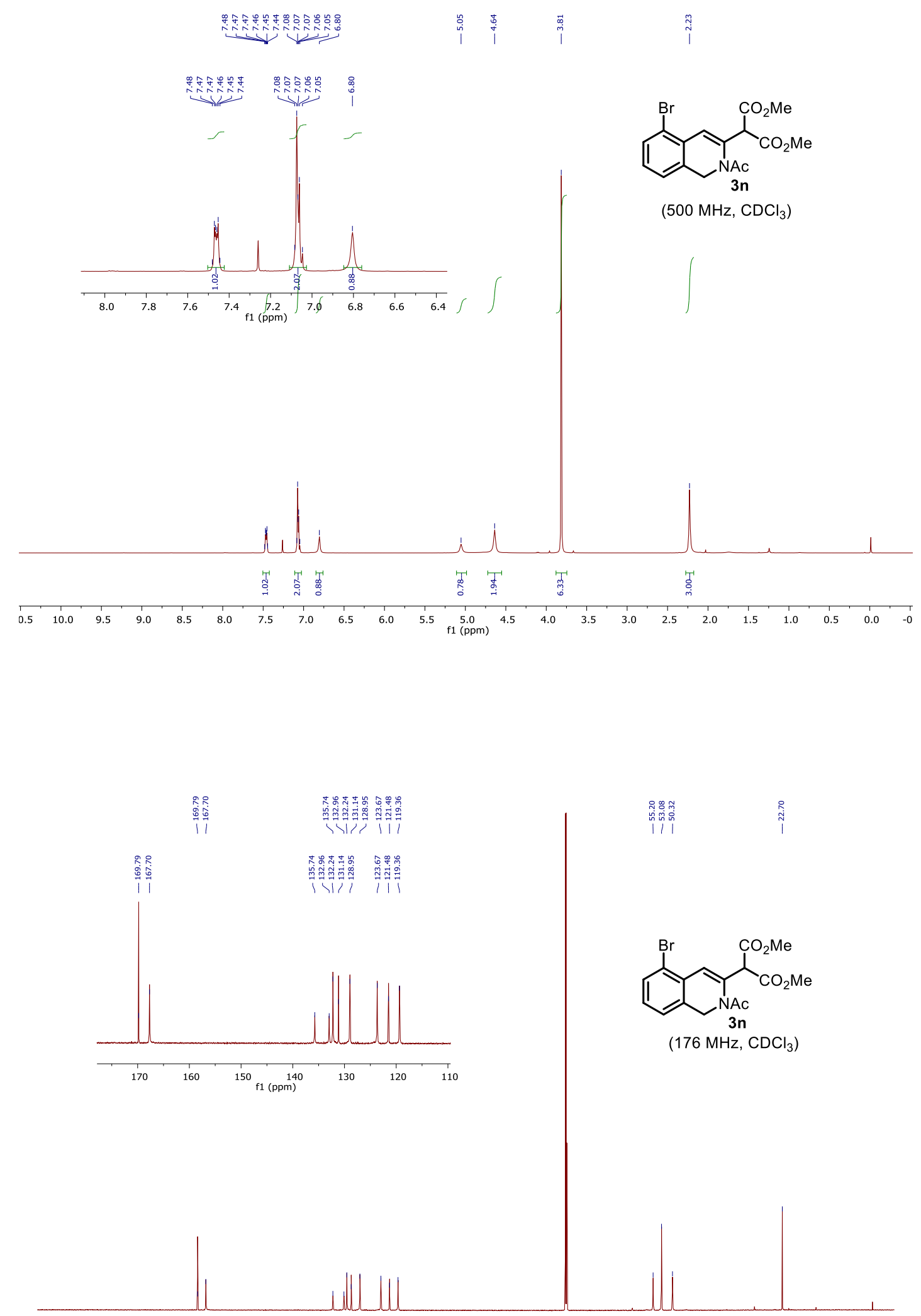

Page S66 of S103 

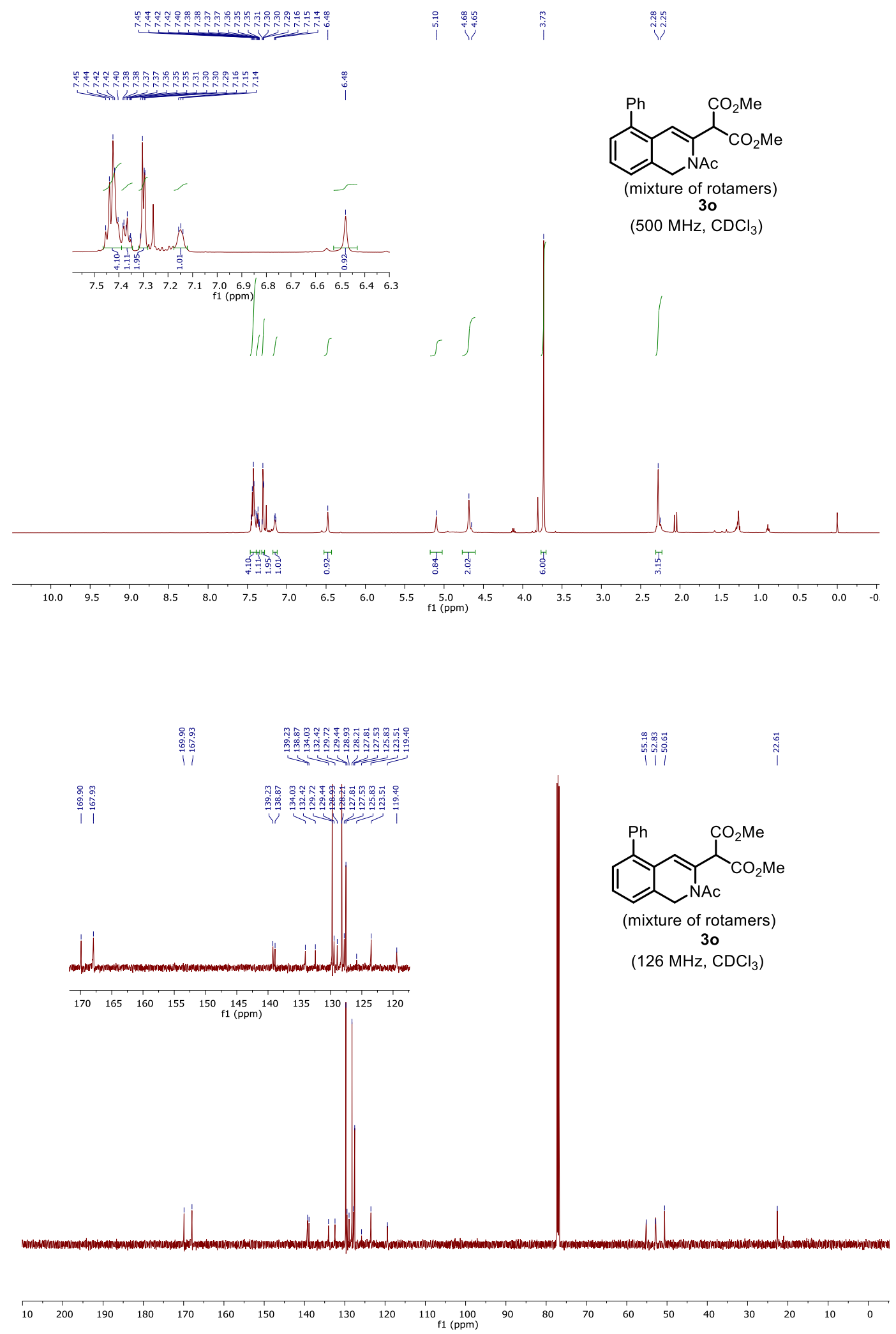

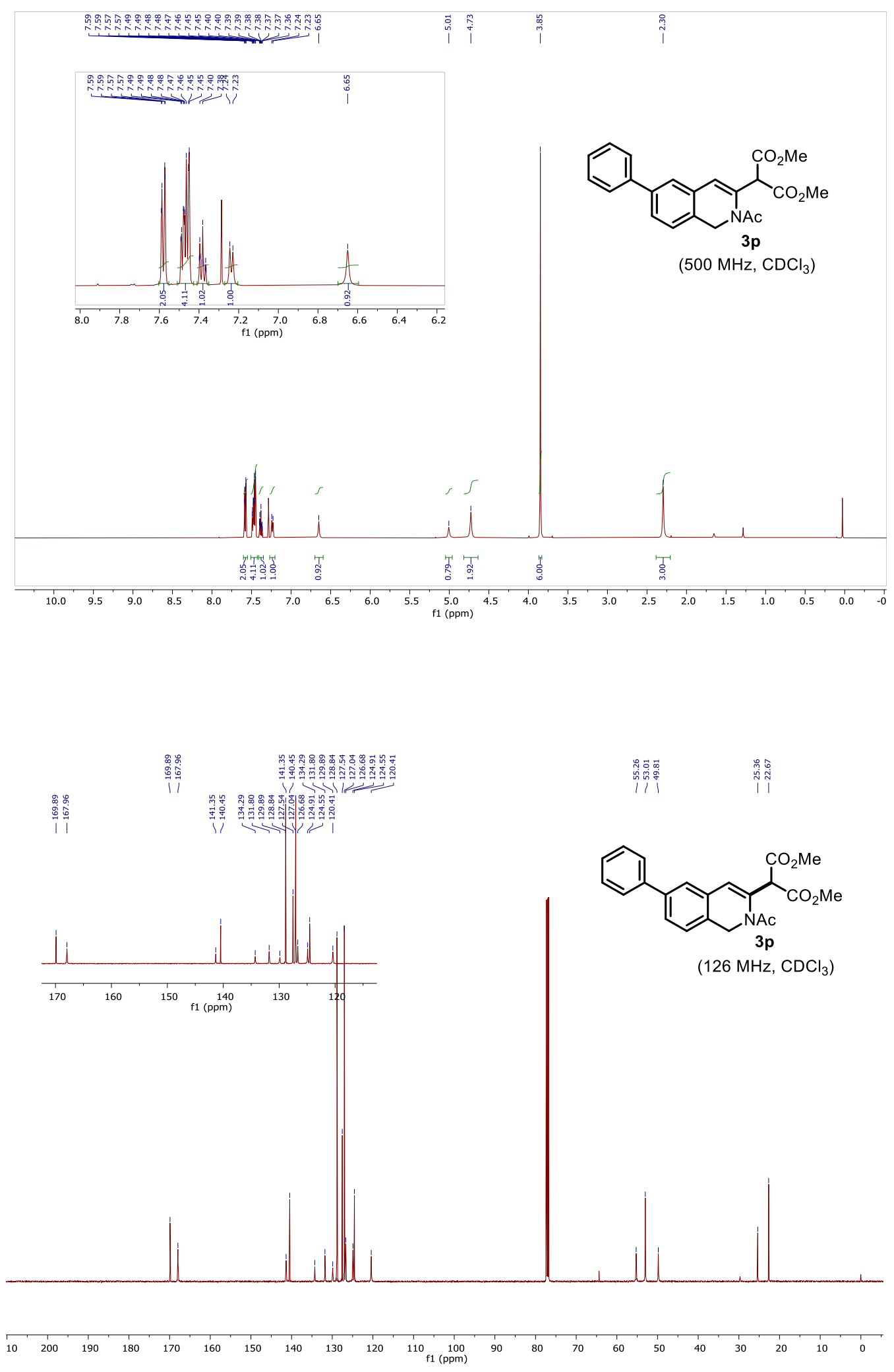

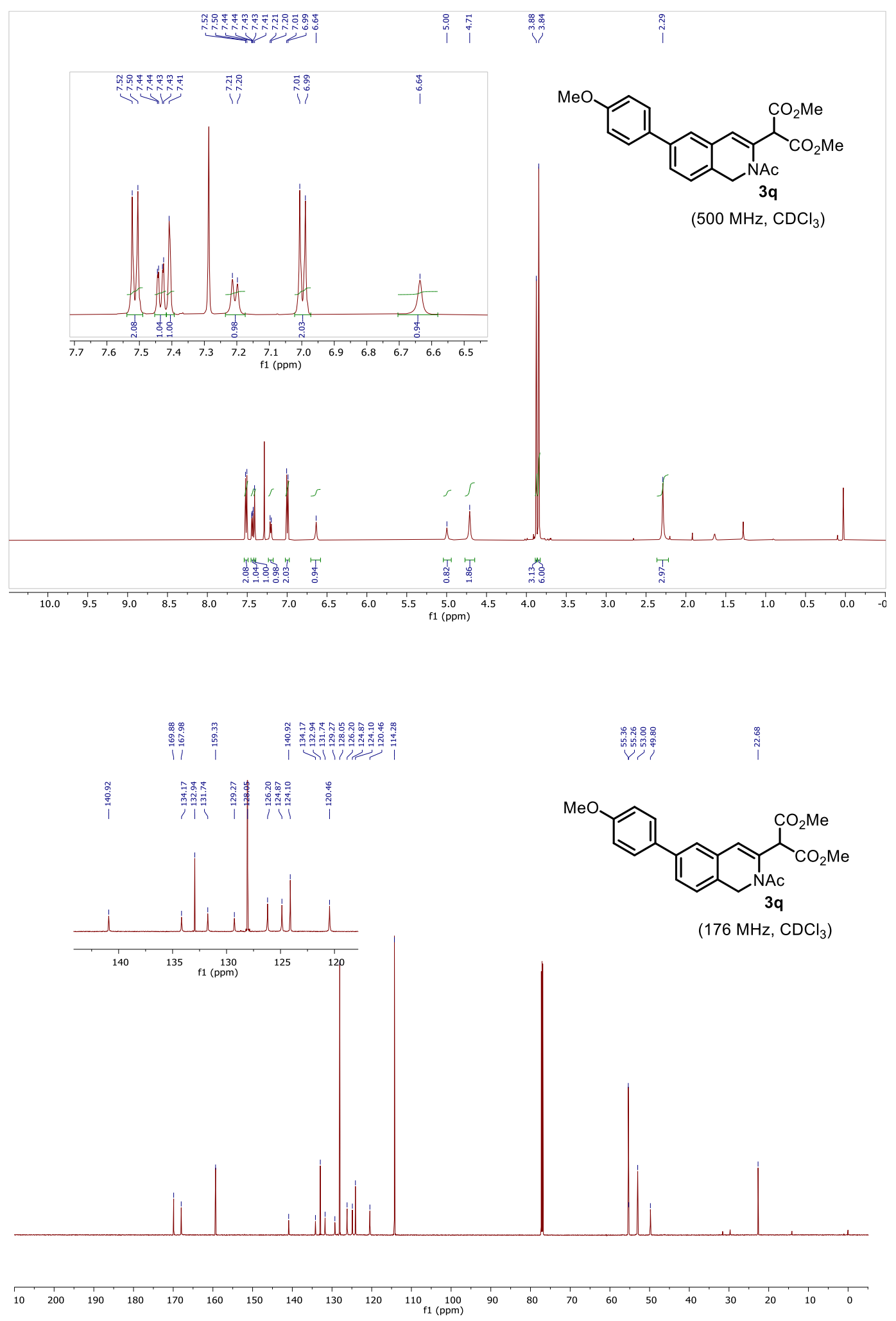


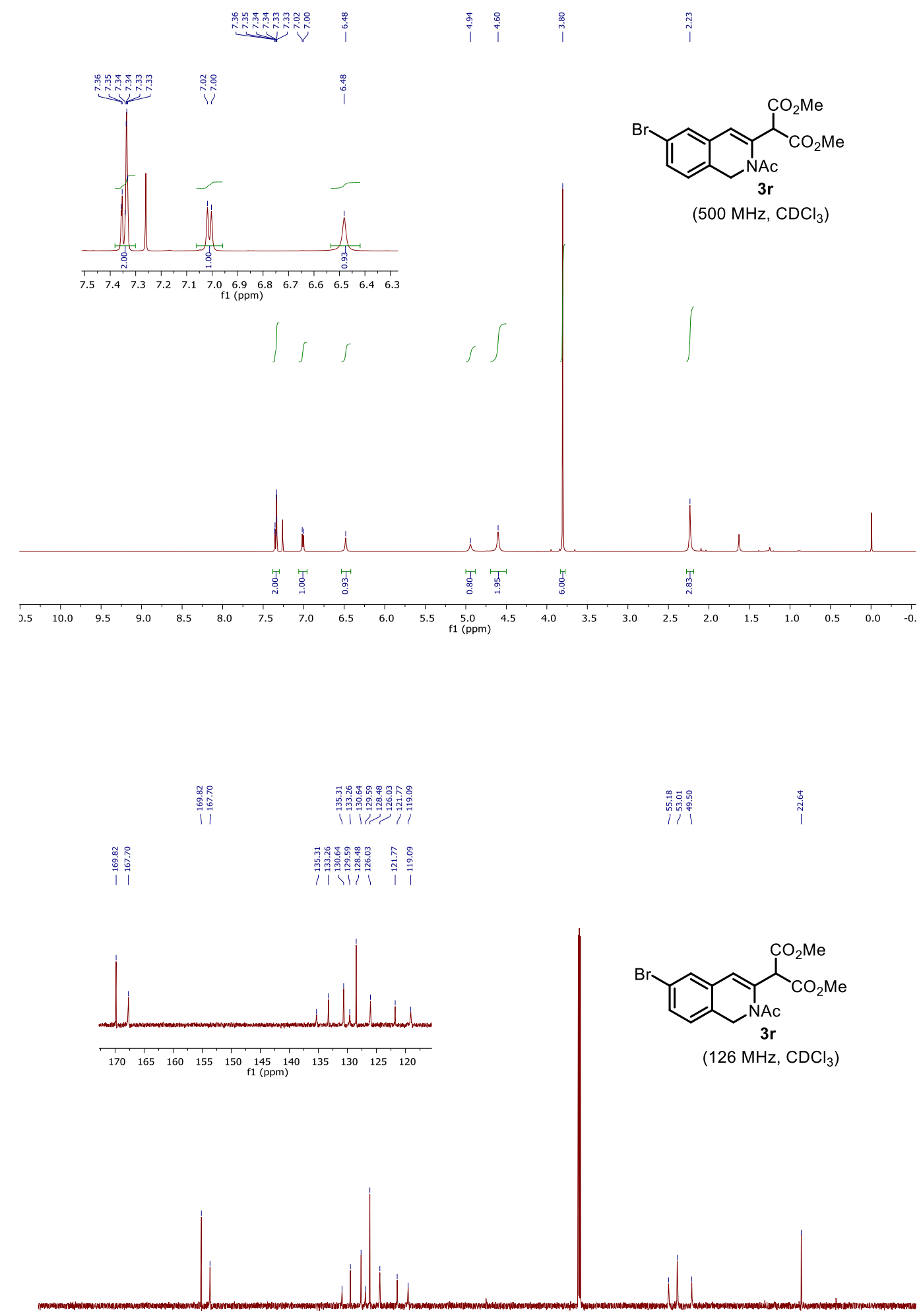

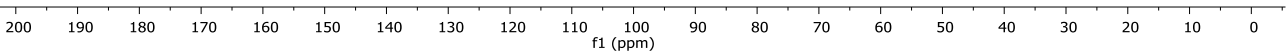



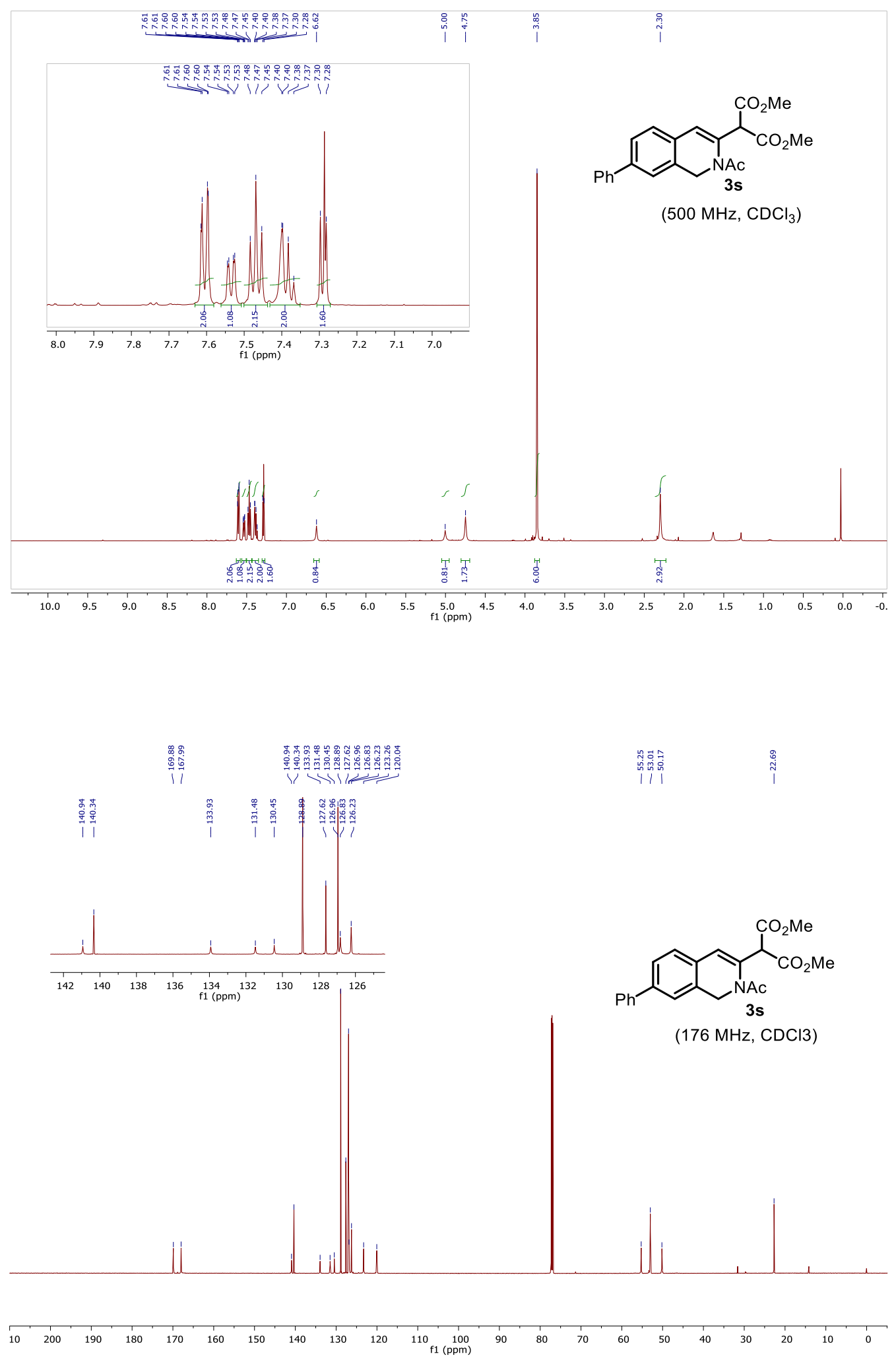

Page S71 of S103 

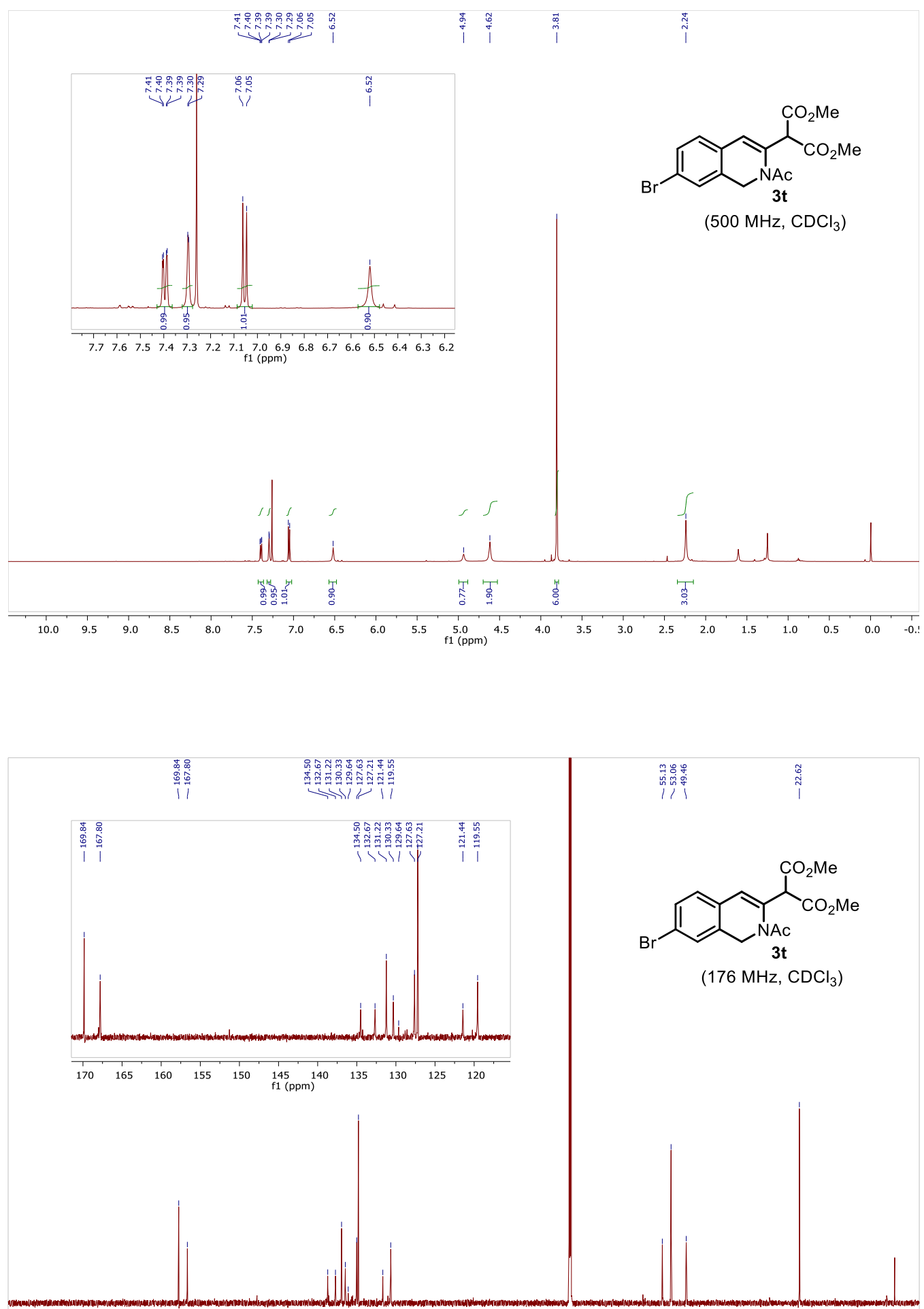

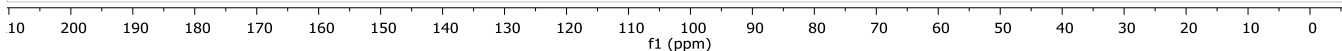



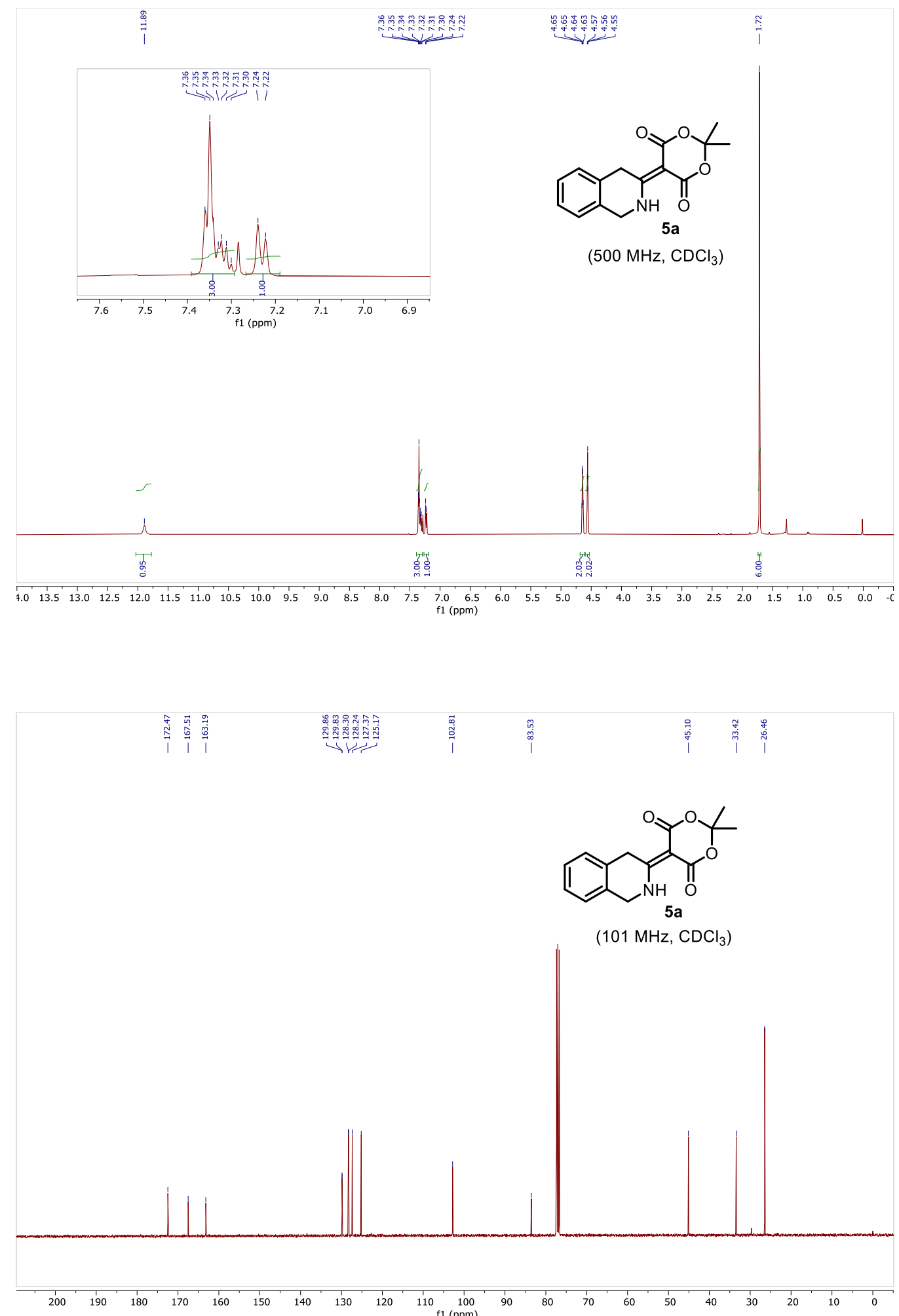

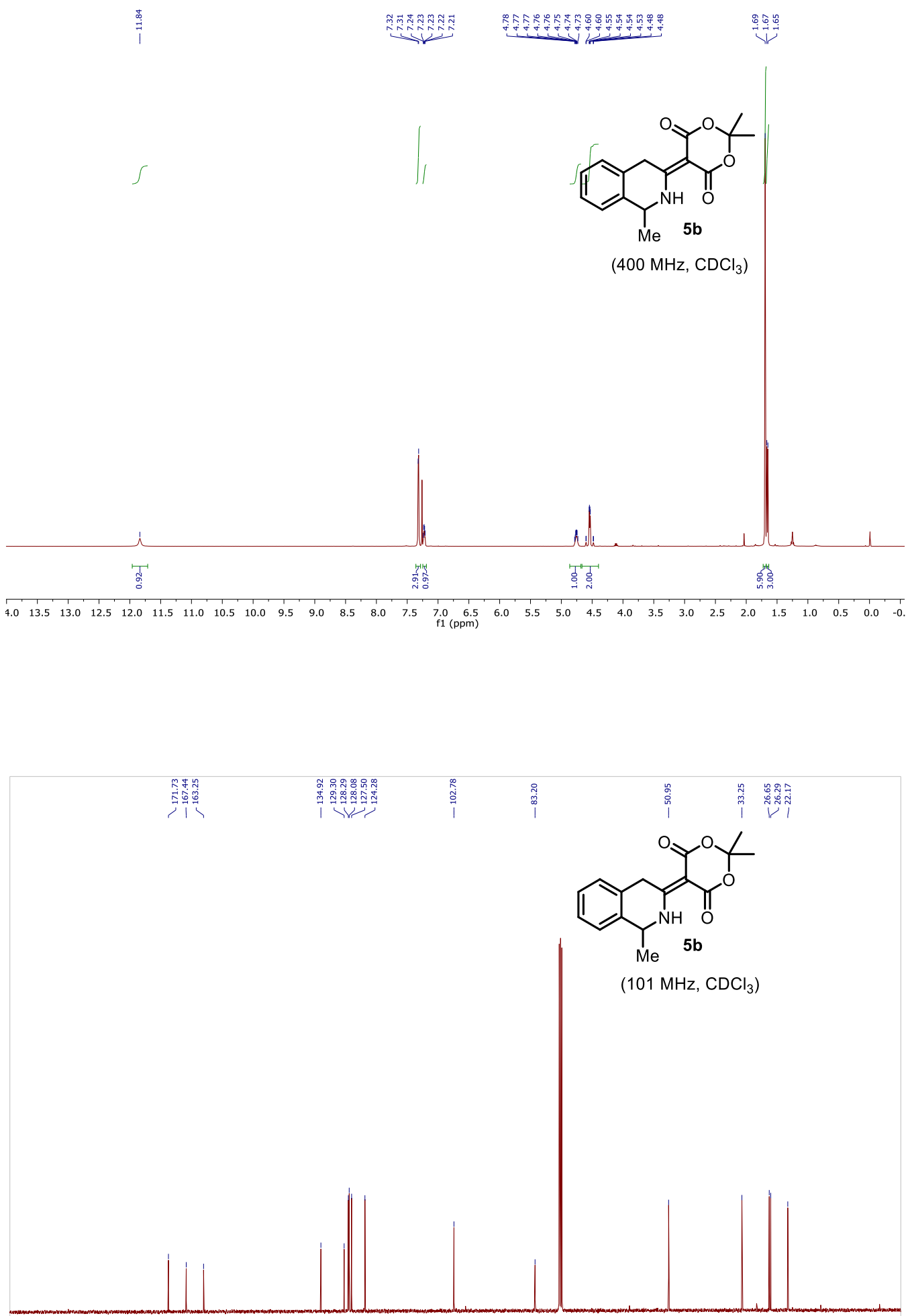

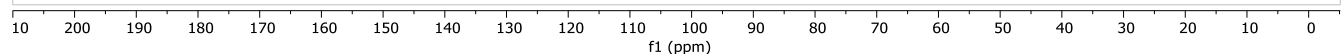




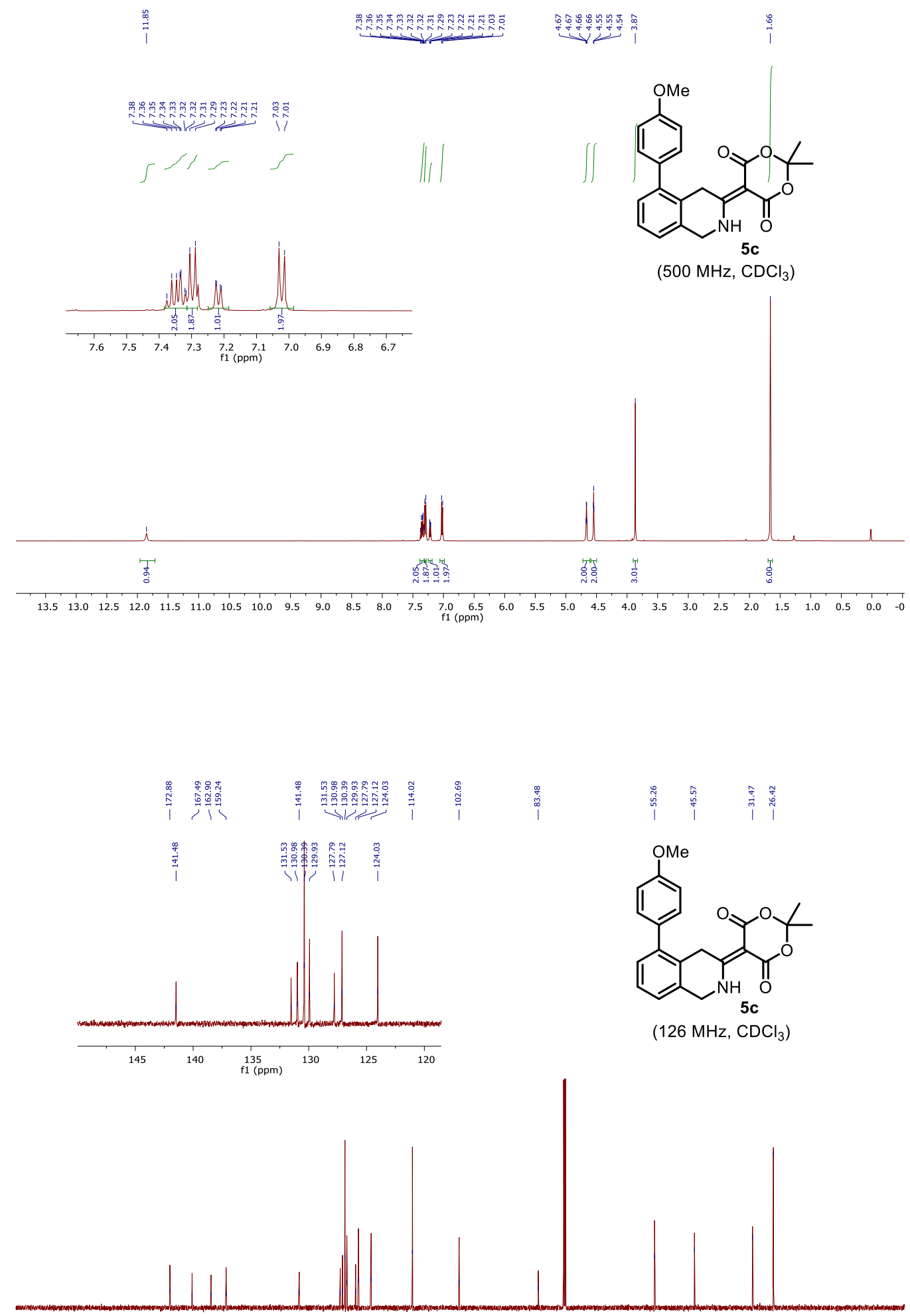

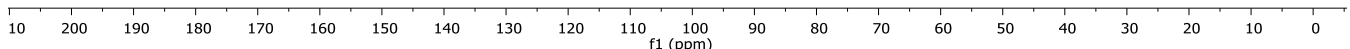



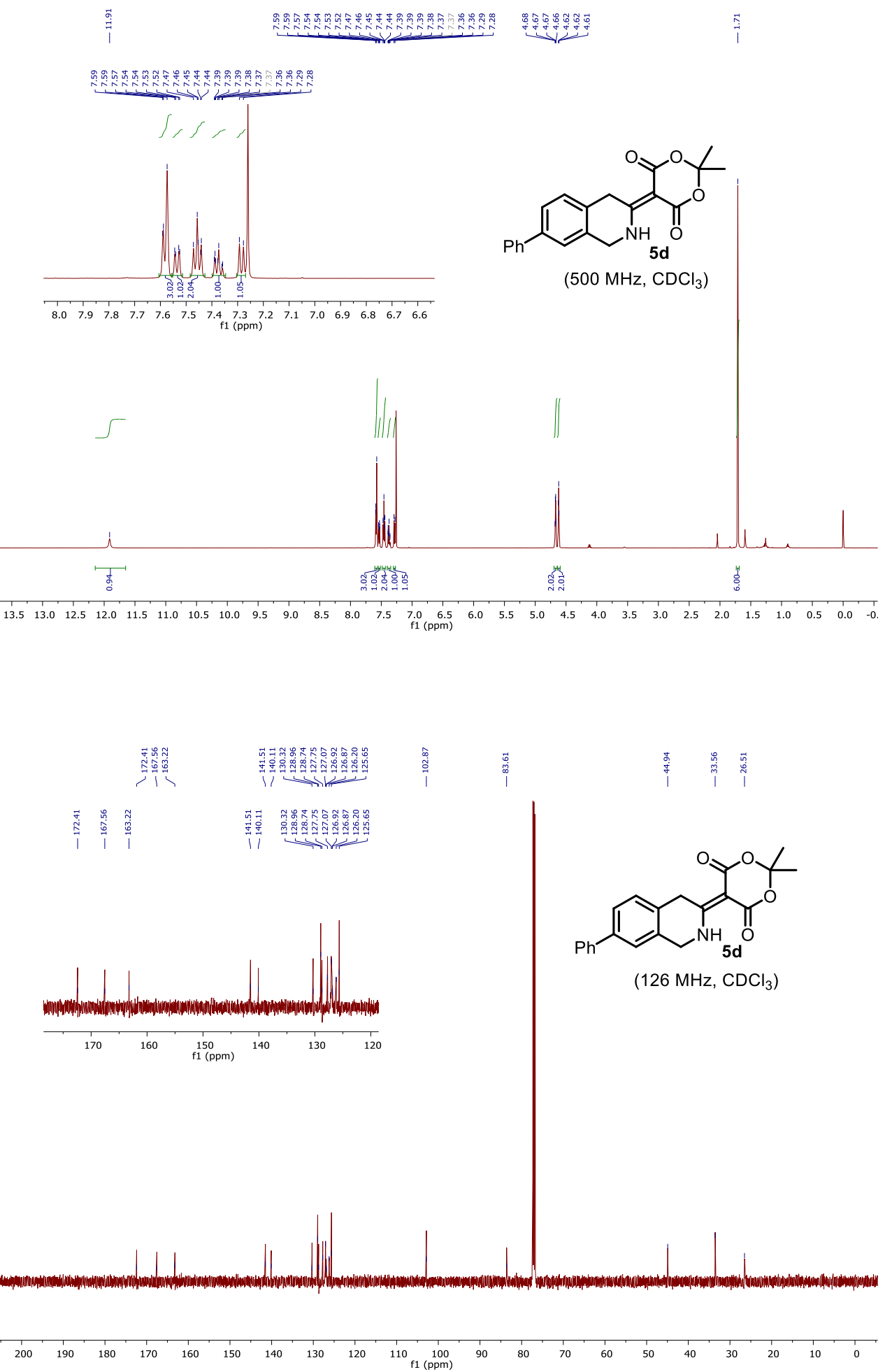

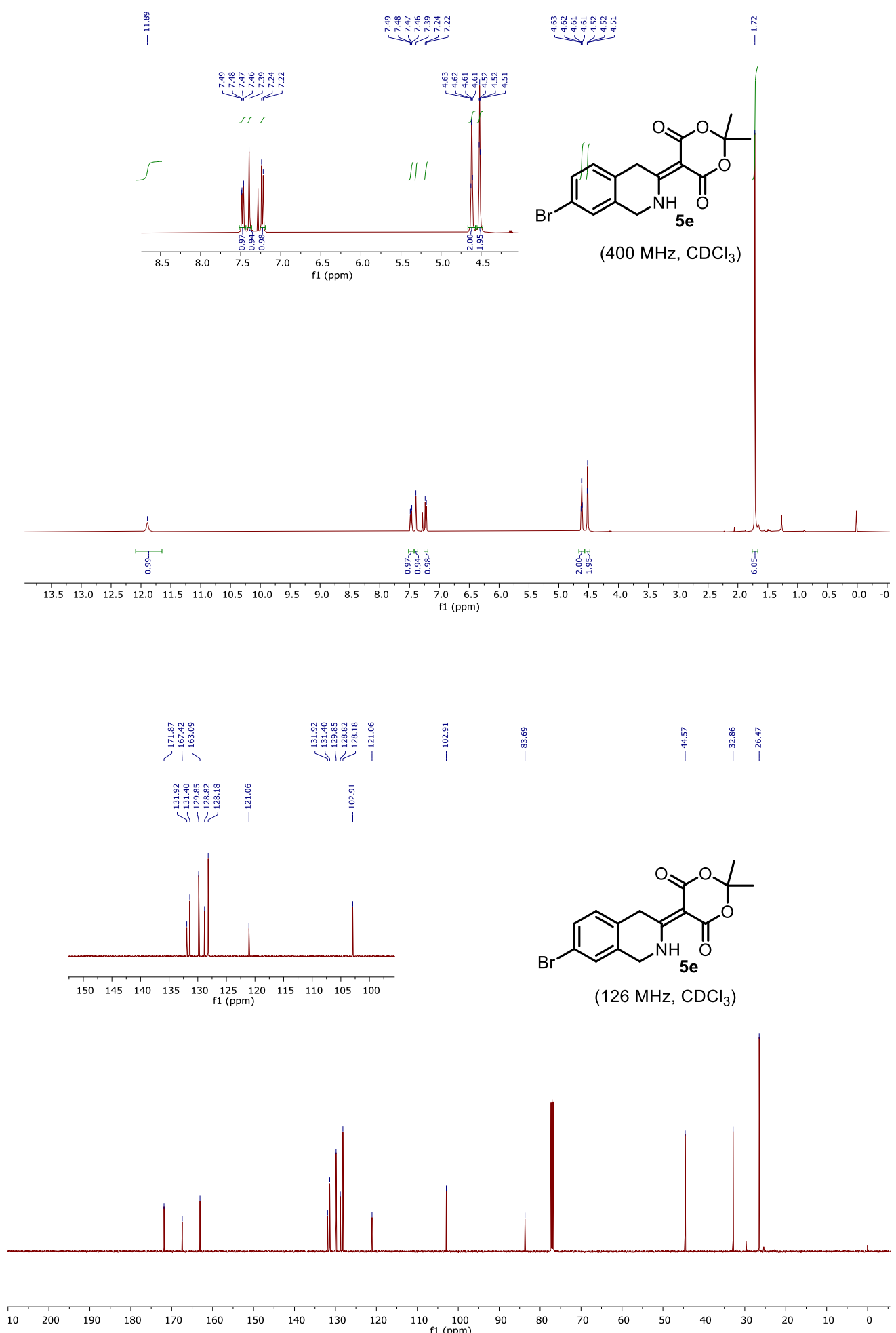


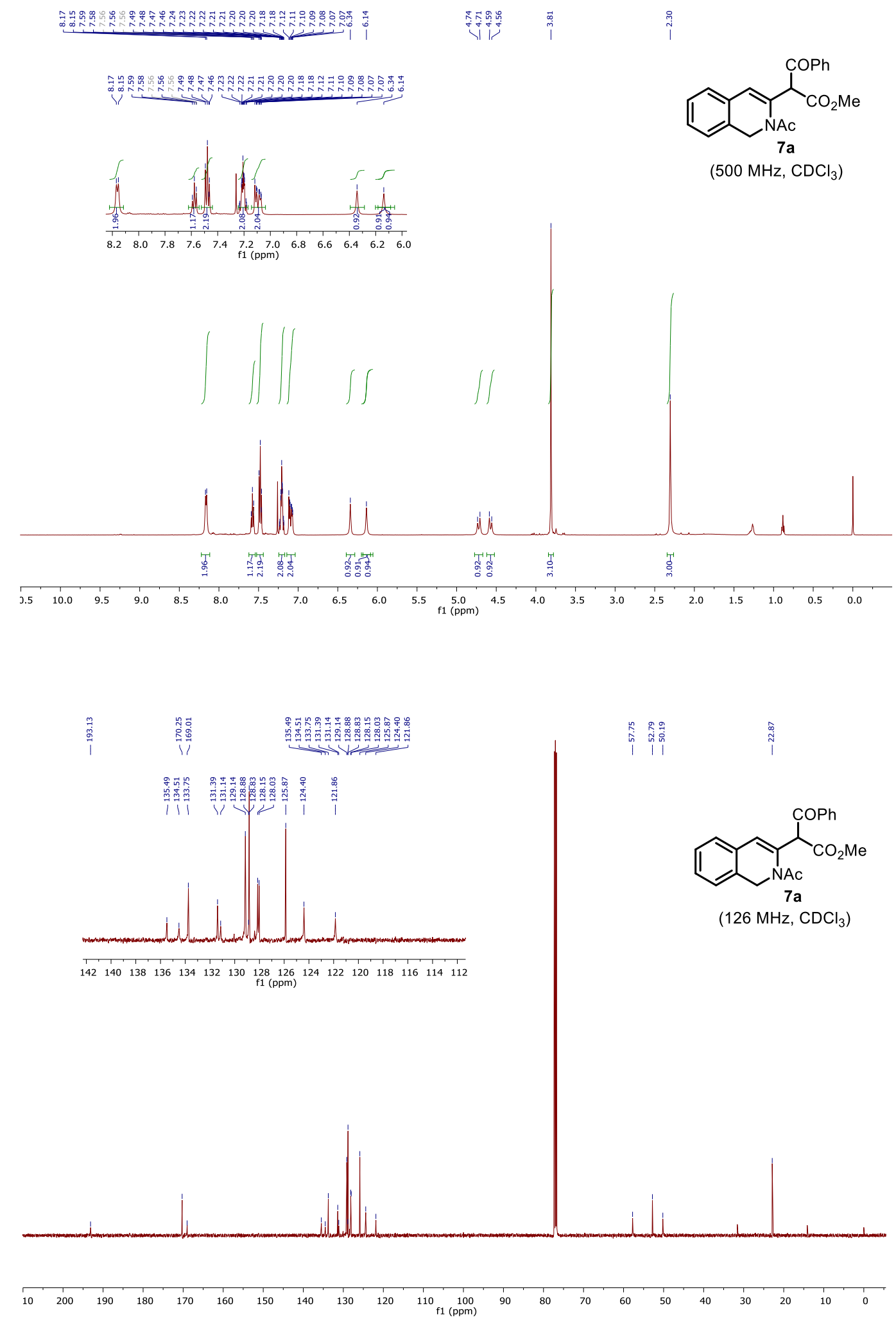



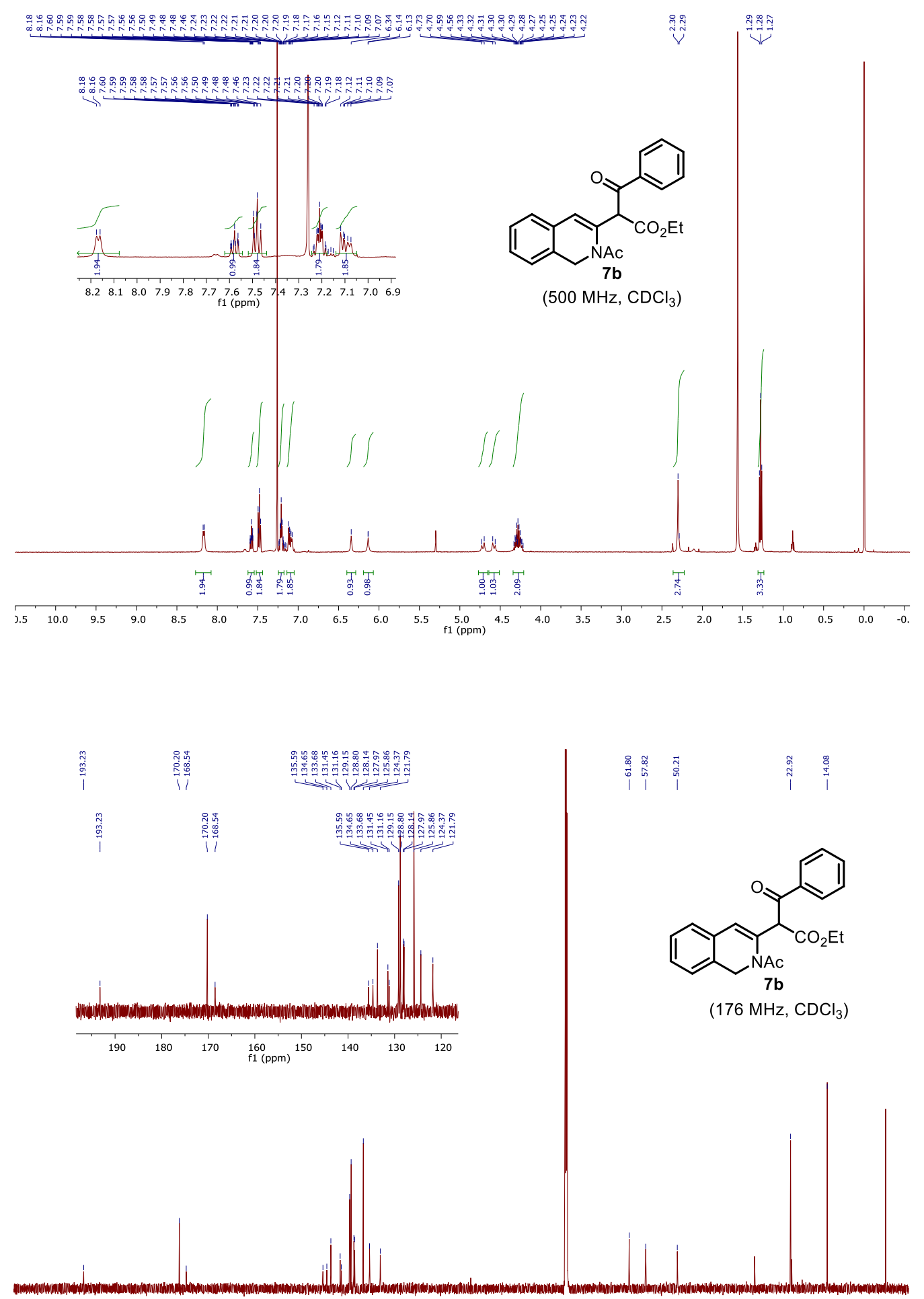

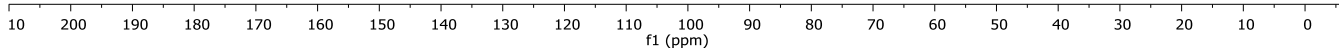




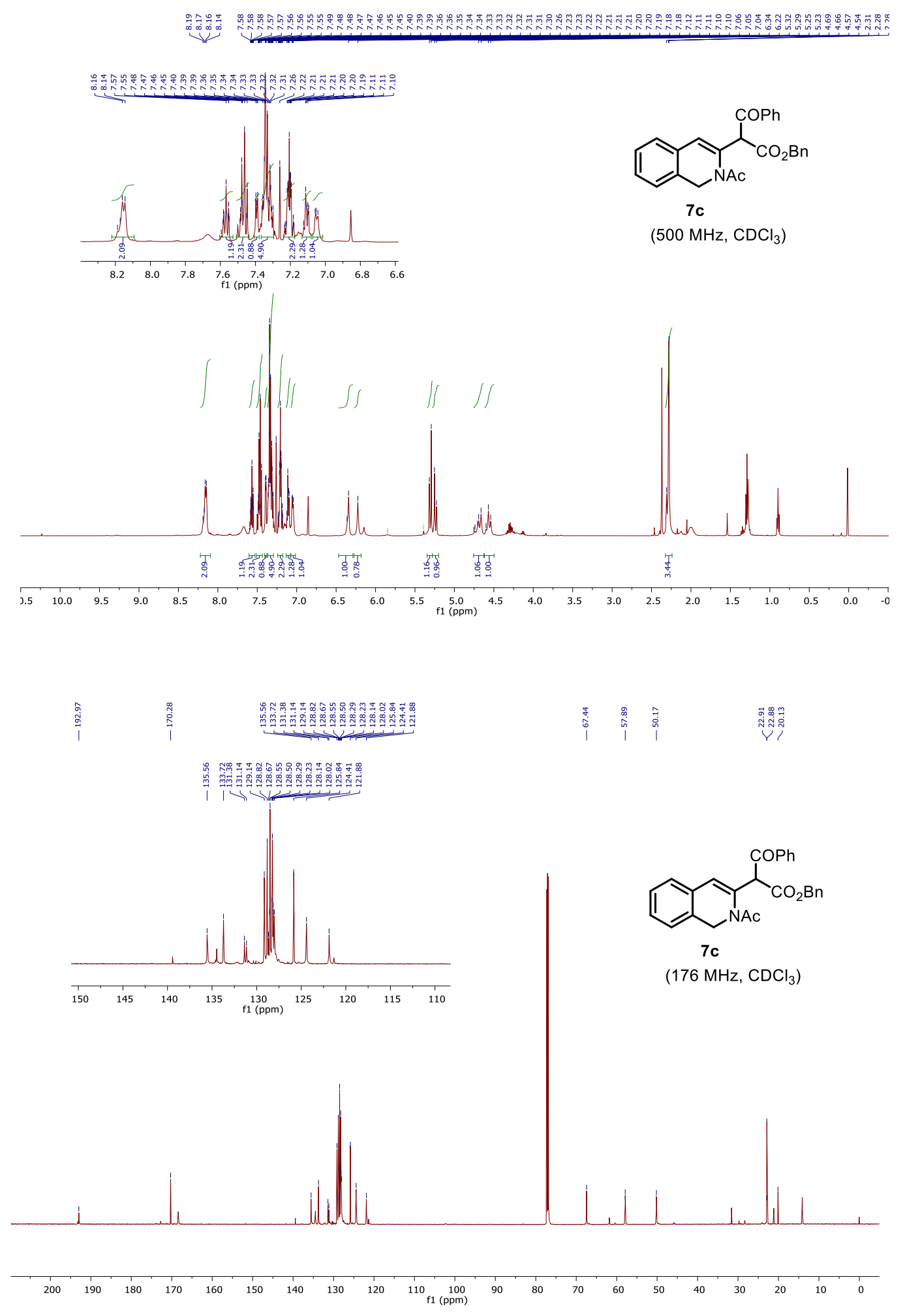



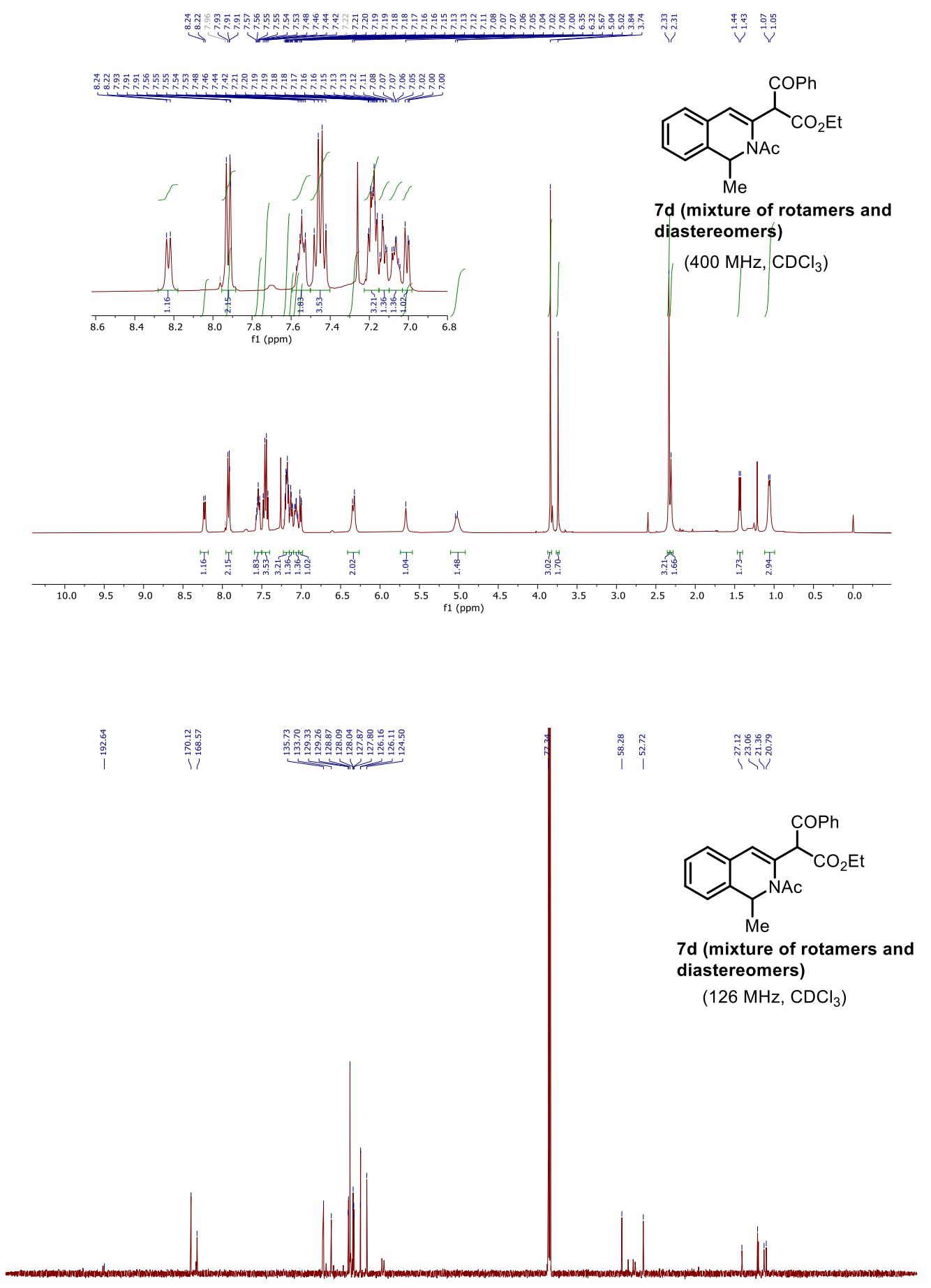

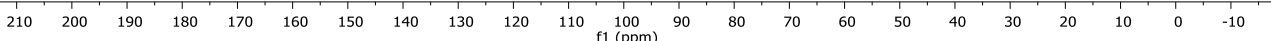




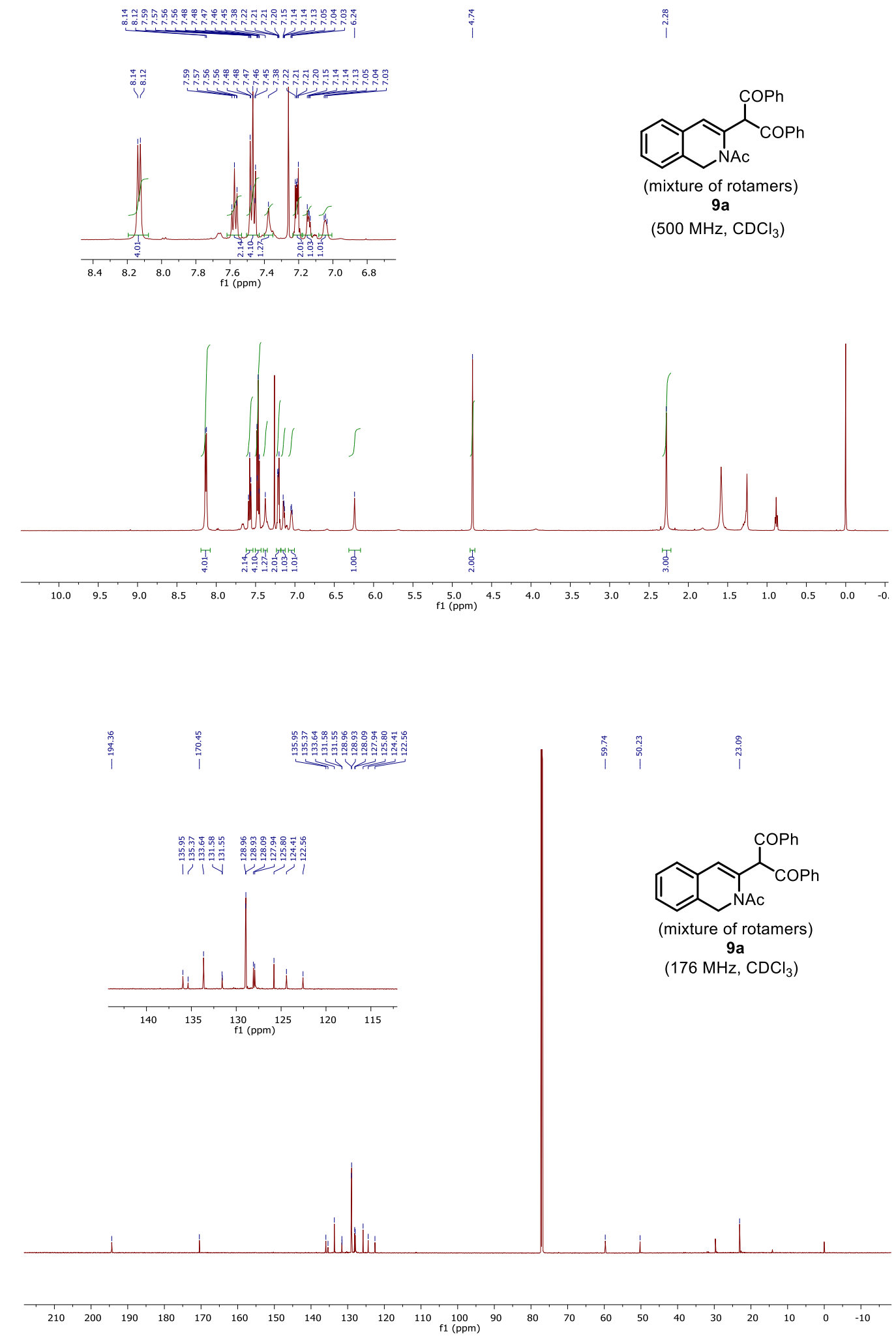

Page S82 of S103 

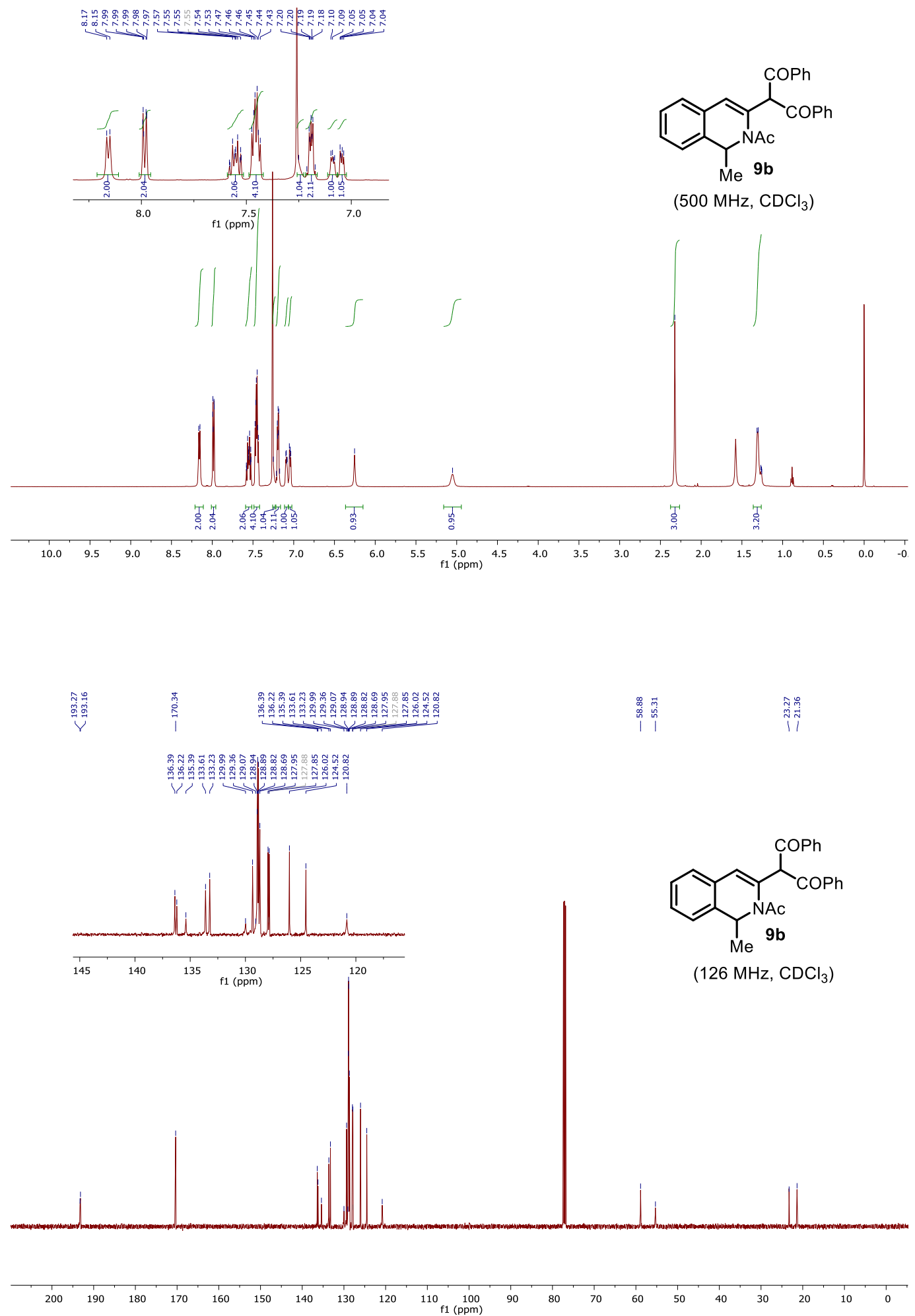

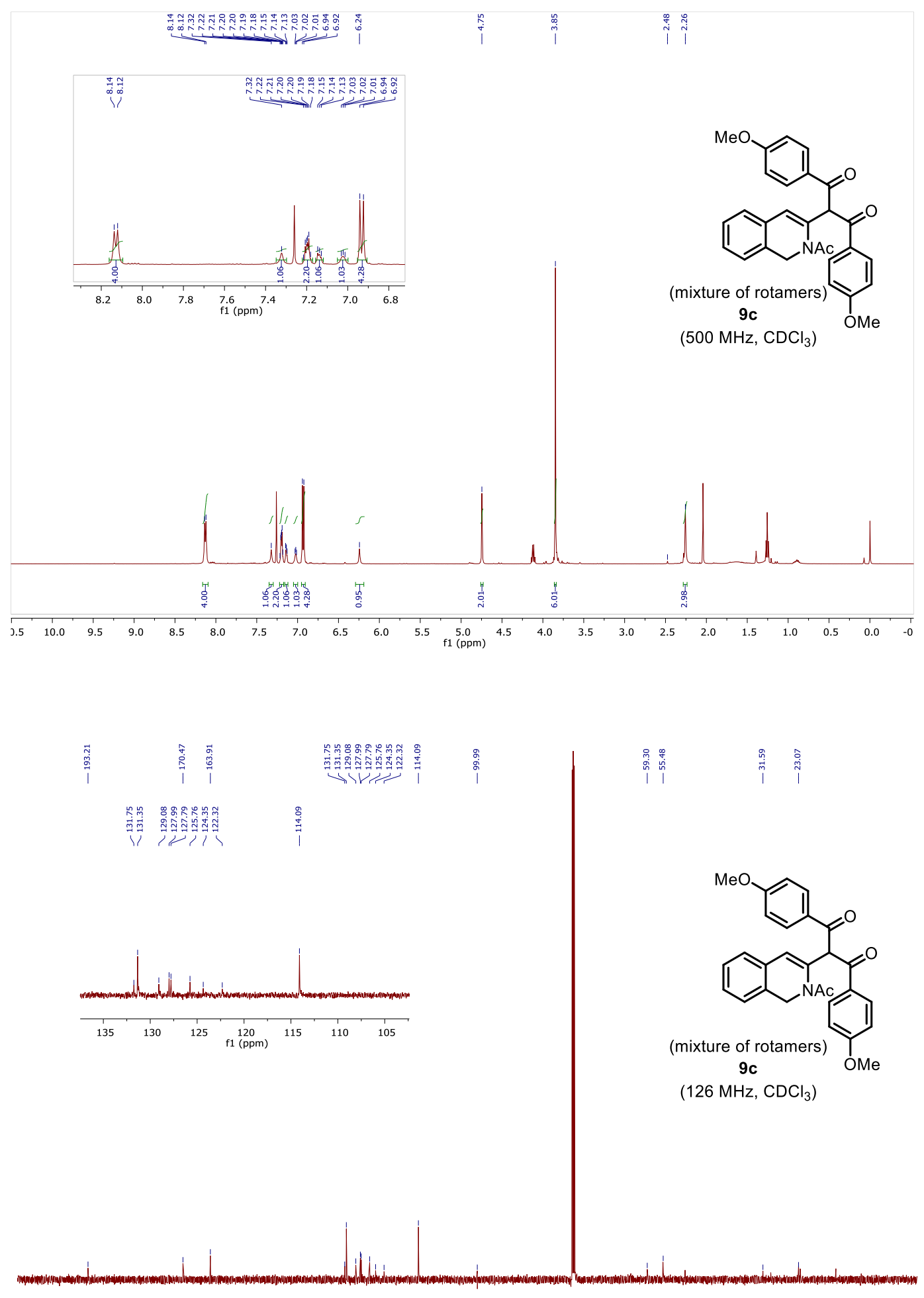

\begin{tabular}{lllllllllllllllllllllll}
\hline 10 & 200 & 190 & 180 & 170 & 160 & 150 & 140 & 130 & 120 & 110 & 100 & 10 & 80 & 70 & 60 & 50 & 40 & 30 & 20 & 10 & 0
\end{tabular} 

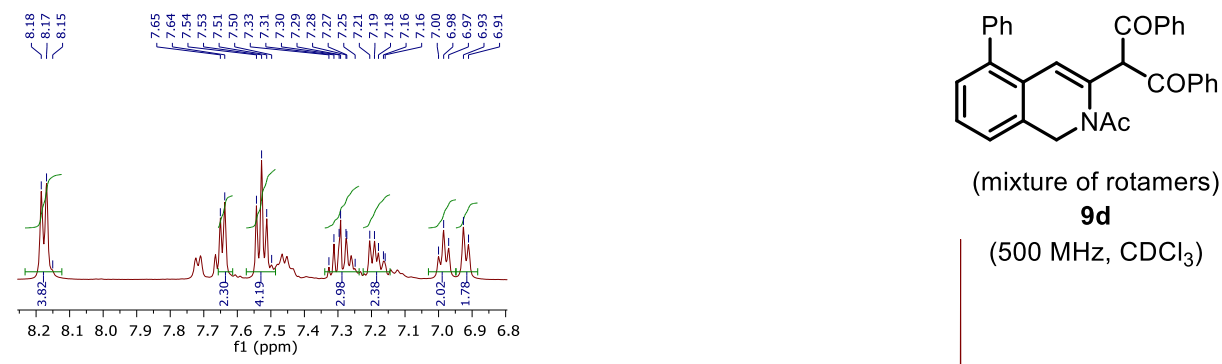

(mixture of rotamers)

9d

(500 MHz, CDCl ${ }_{3}$ )
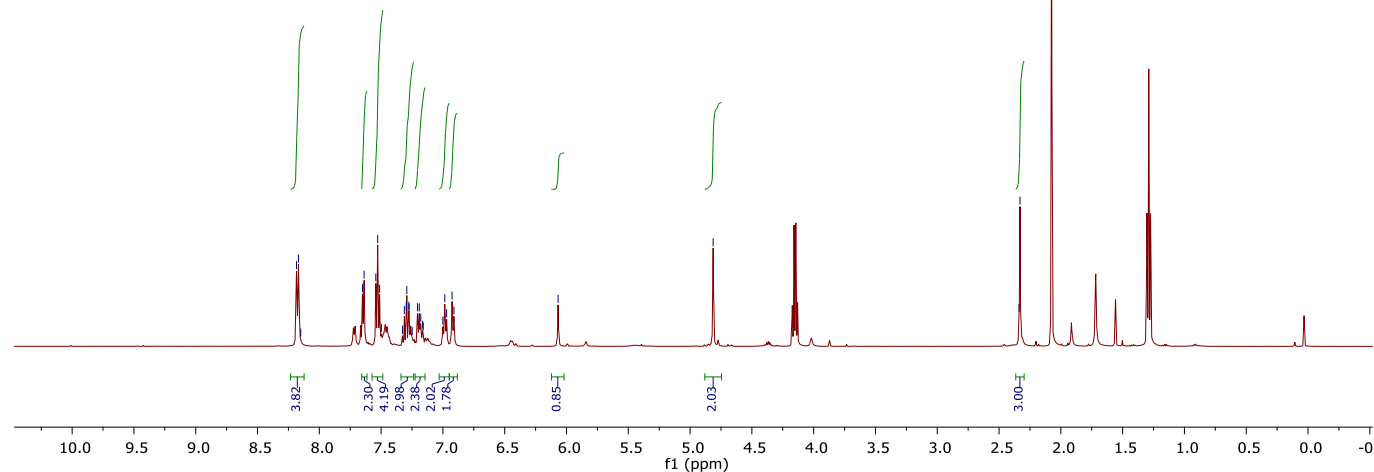

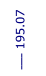

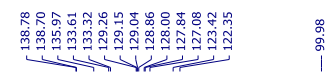

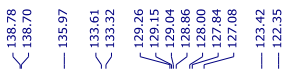
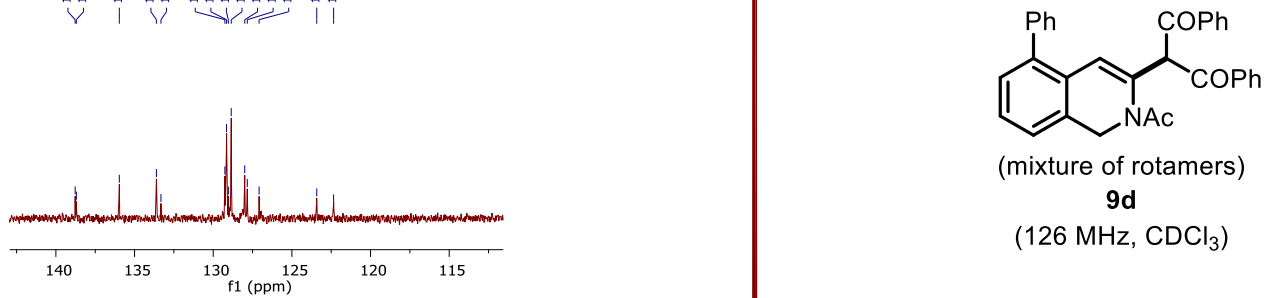

(mixture of rotamers)

9d

(126 MHz, $\mathrm{CDCl}_{3}$ )

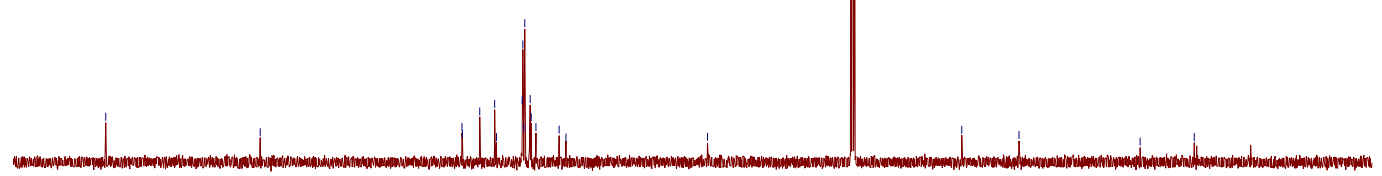

$\begin{array}{llllllllllllllllllllllllllllll} & 1 & 190 & 180 & 170 & 160 & 150 & 140 & 130 & 120 & 110 & 100 & 90 & 80 & 70 & 60 & 50 & 40 & 30 & 20 & 10 & 0\end{array}$ 


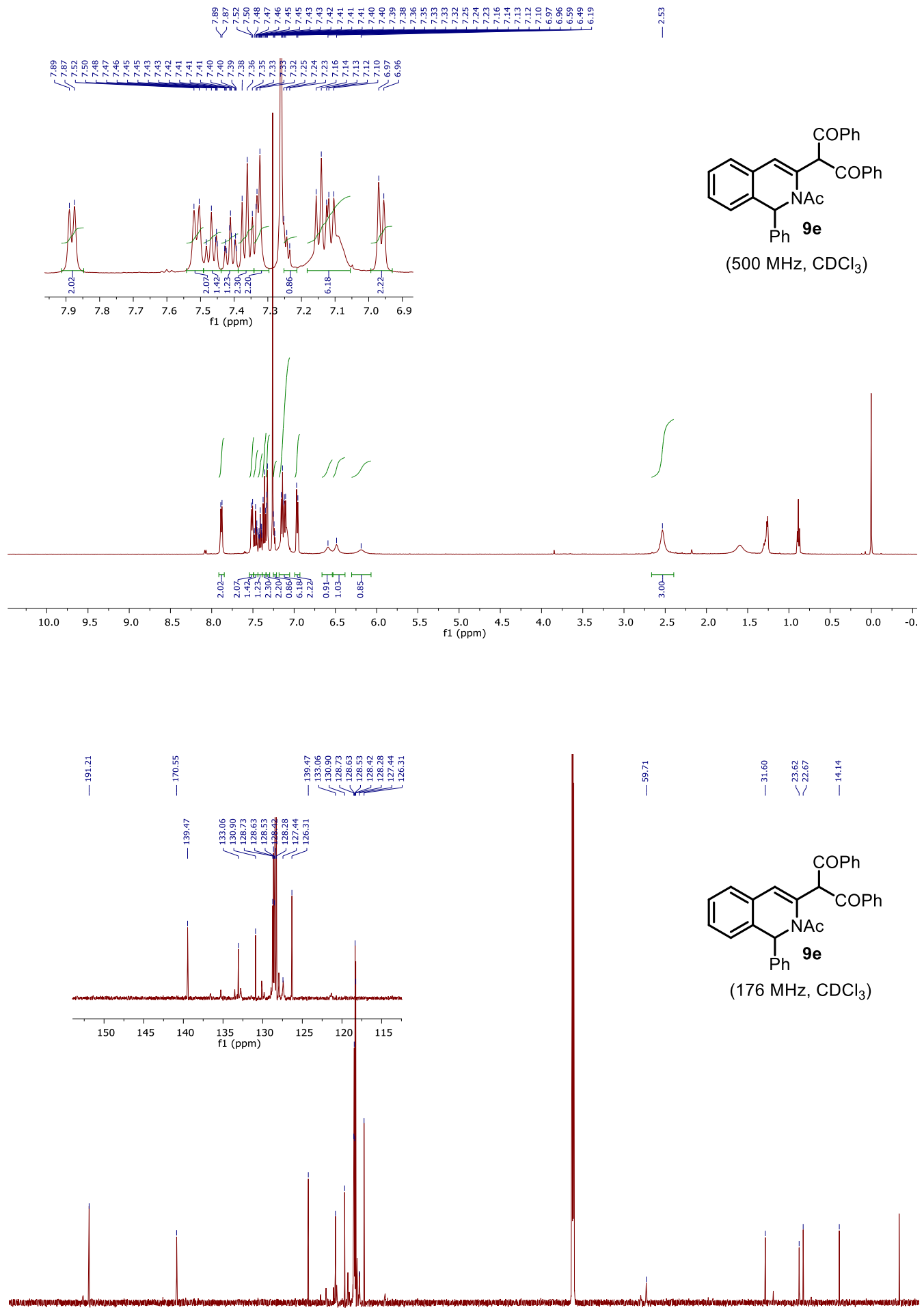

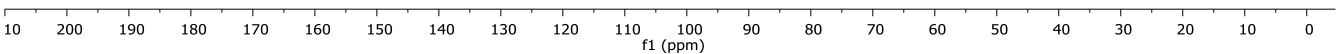



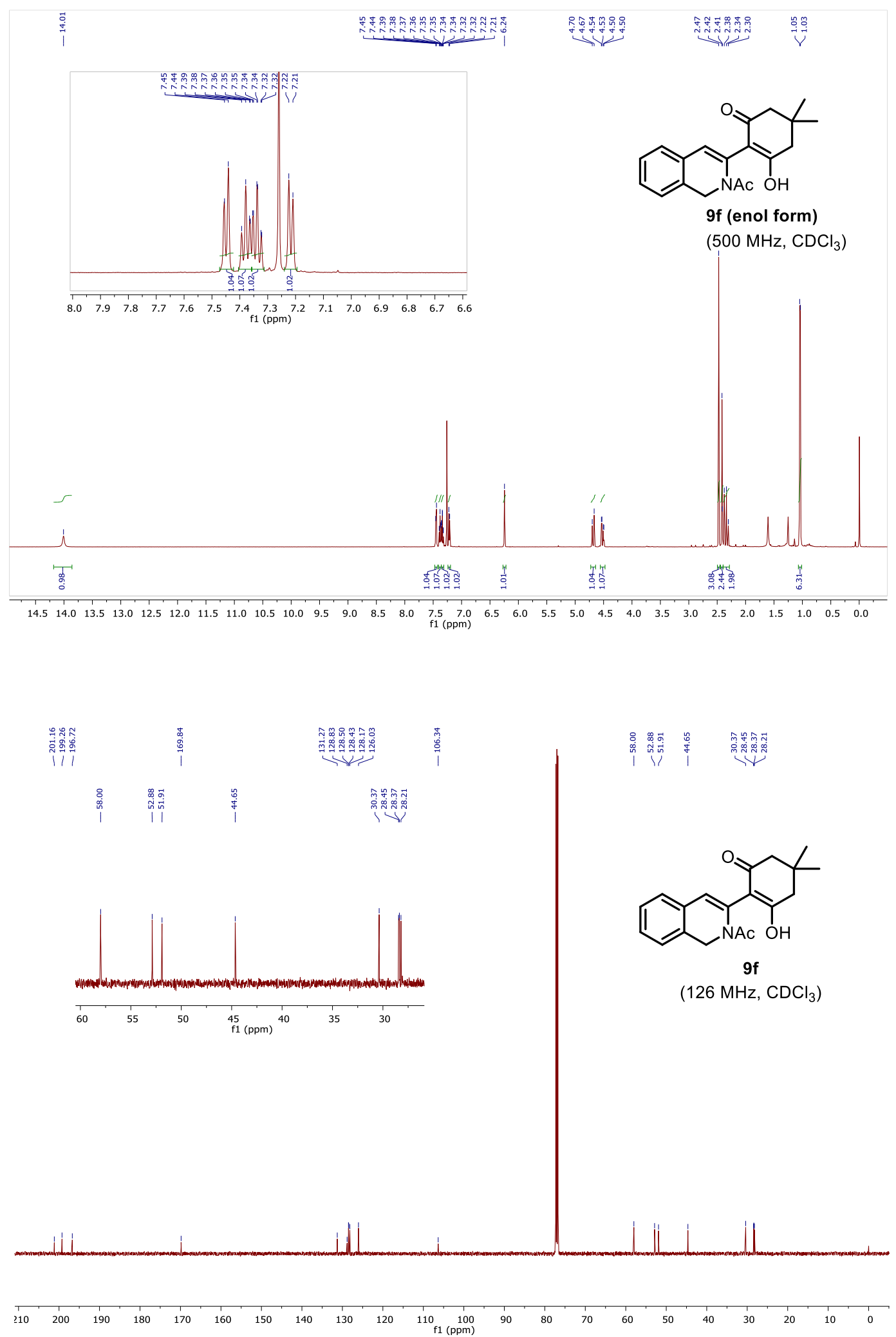


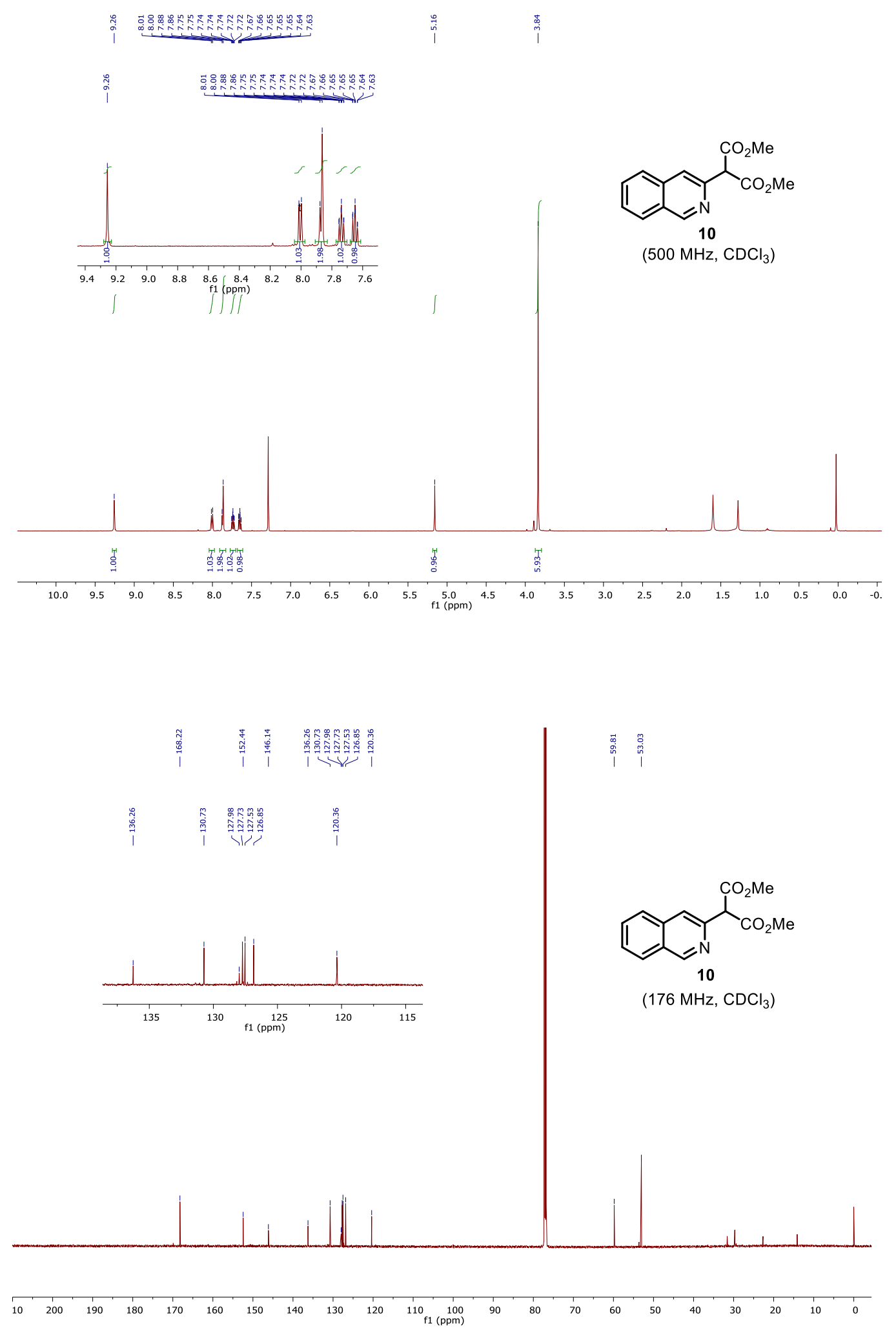

Page S88 of S103 

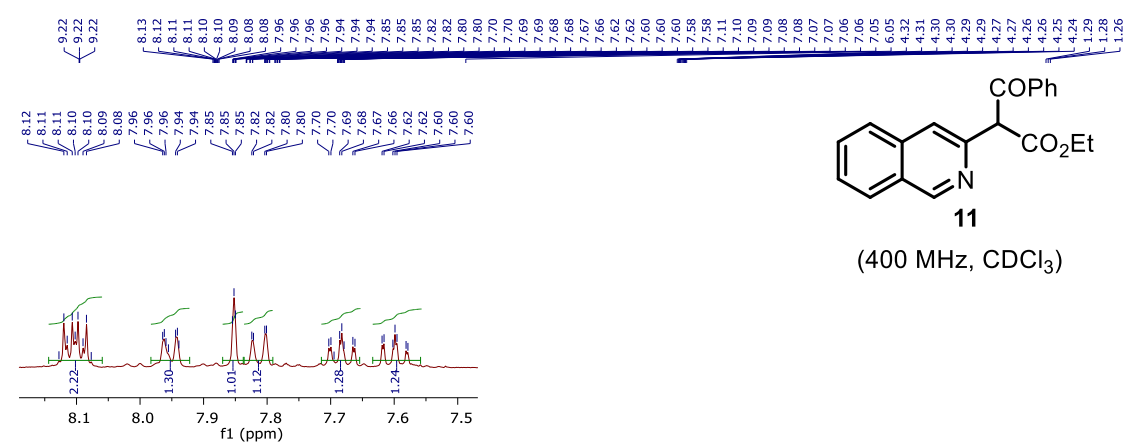

$\left(400 \mathrm{MHz}, \mathrm{CDCl}_{3}\right)$

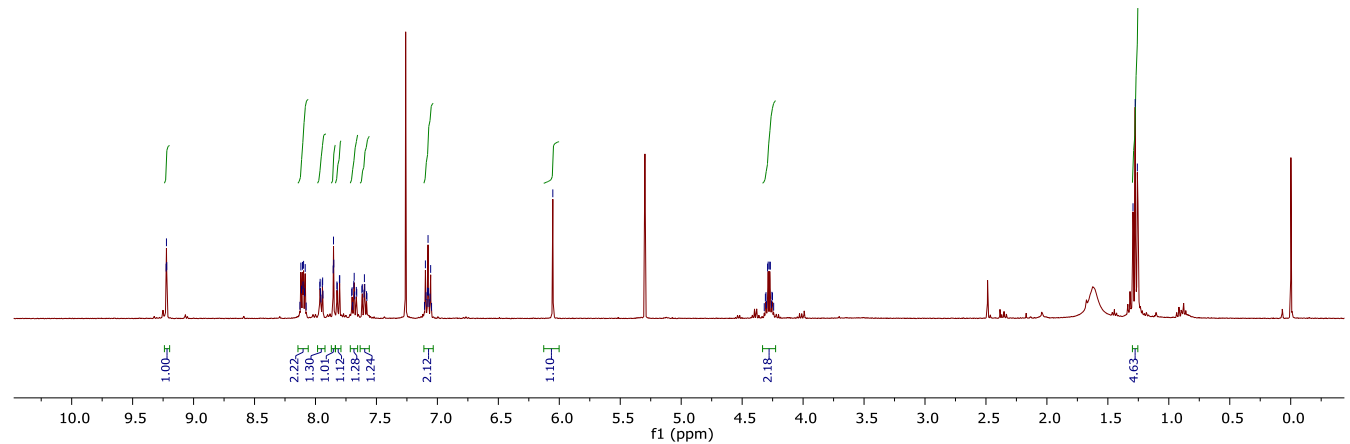

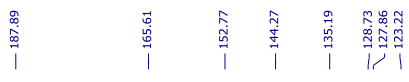
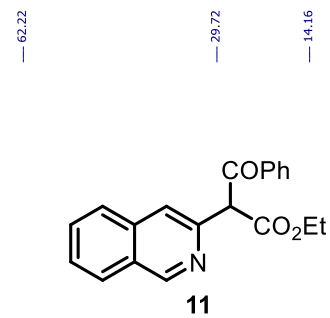

(176 MHz, $\mathrm{CDCl}_{3}$ )

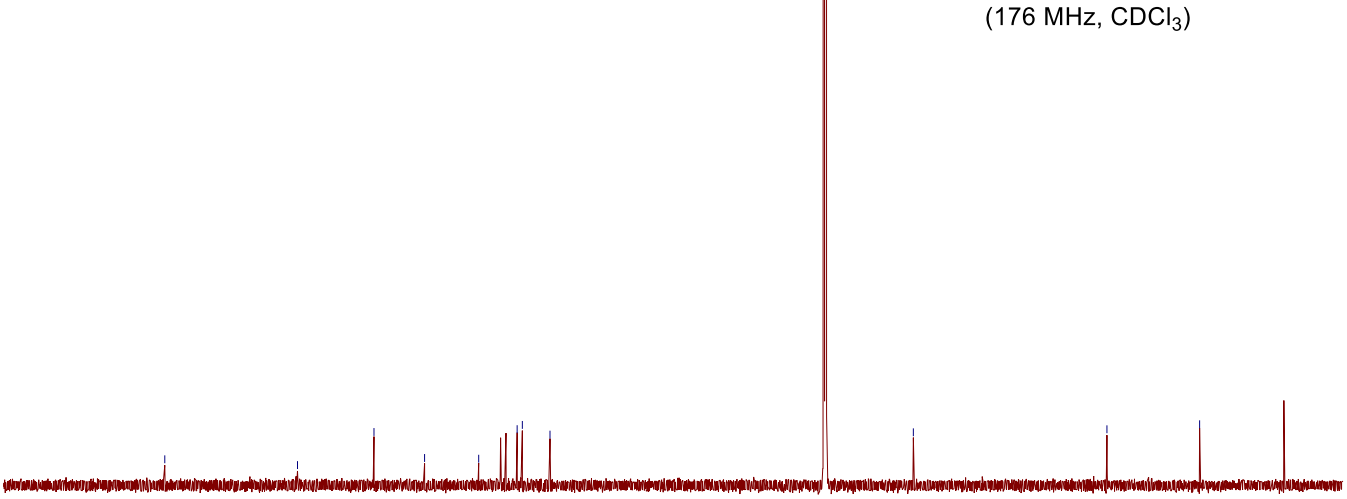

$\begin{array}{lllllllllllllllllllllllllllllllllll}210 & 200 & 190 & 180 & 170 & 160 & 150 & 140 & 130 & 120 & 110 & 100 & 90 & 80 & 70 & 60 & 50 & 40 & 30 & 20 & 10 & 0 & \end{array}$ 


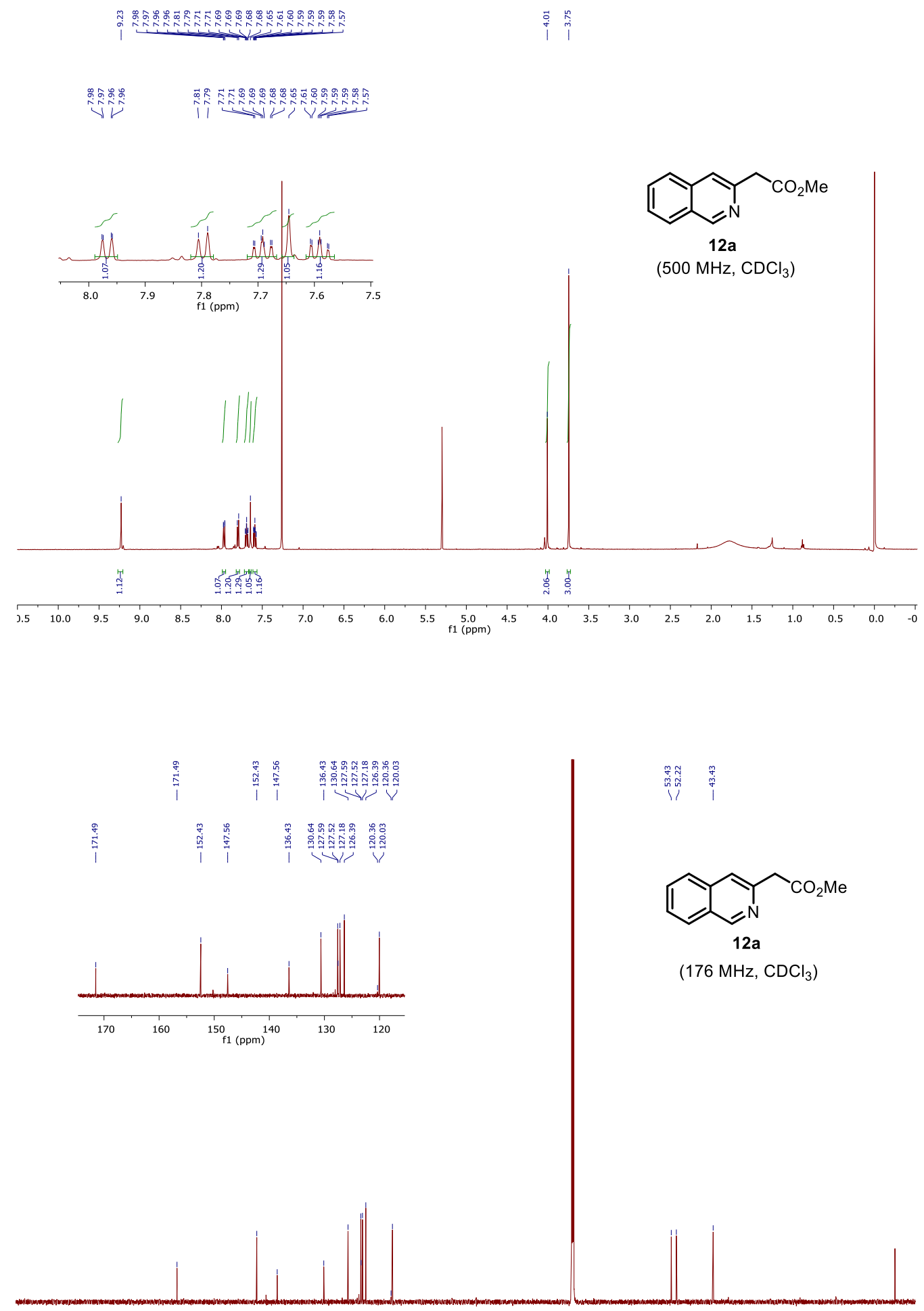

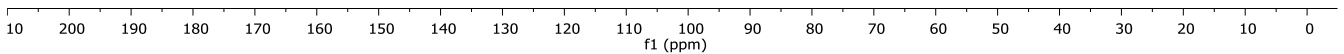



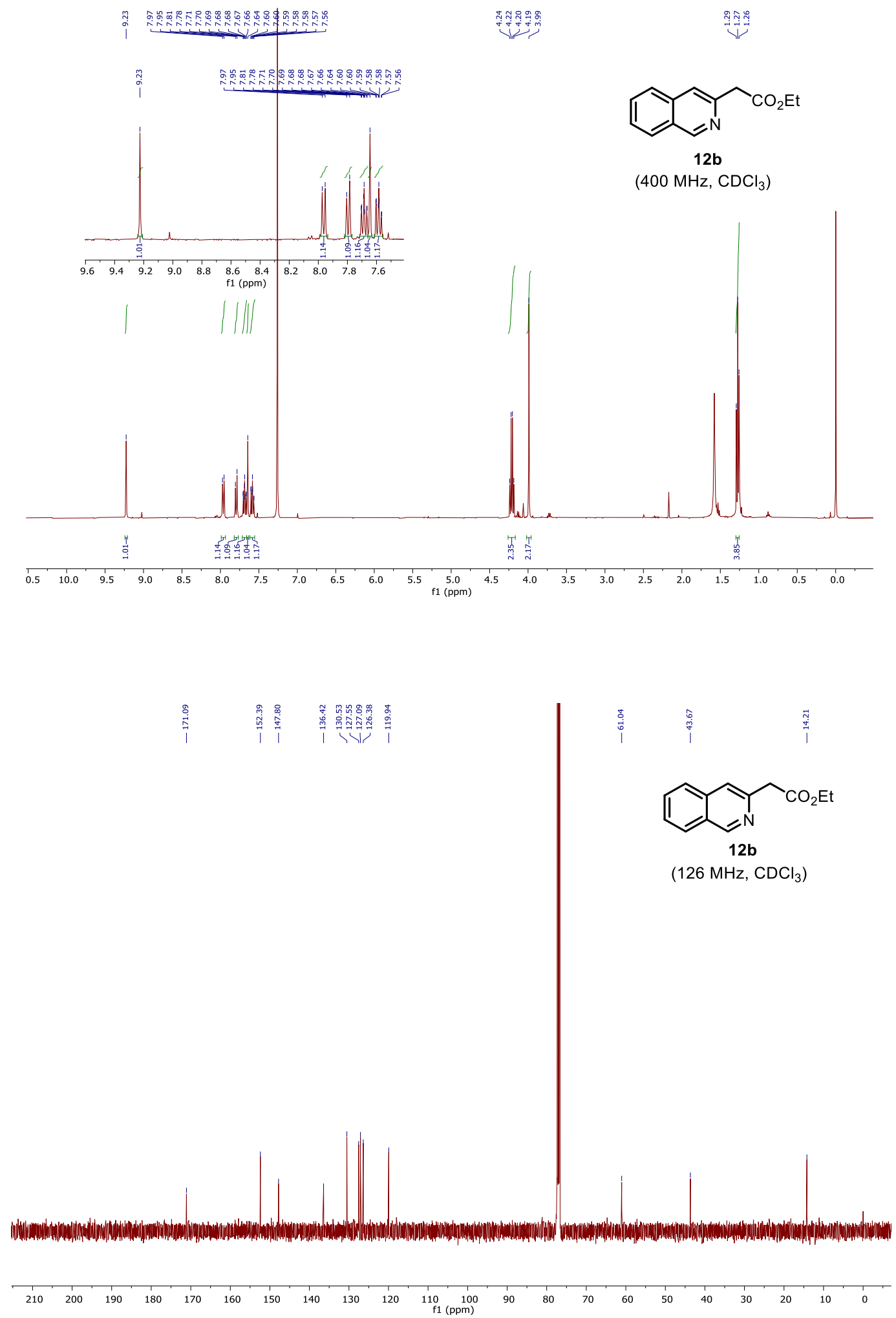


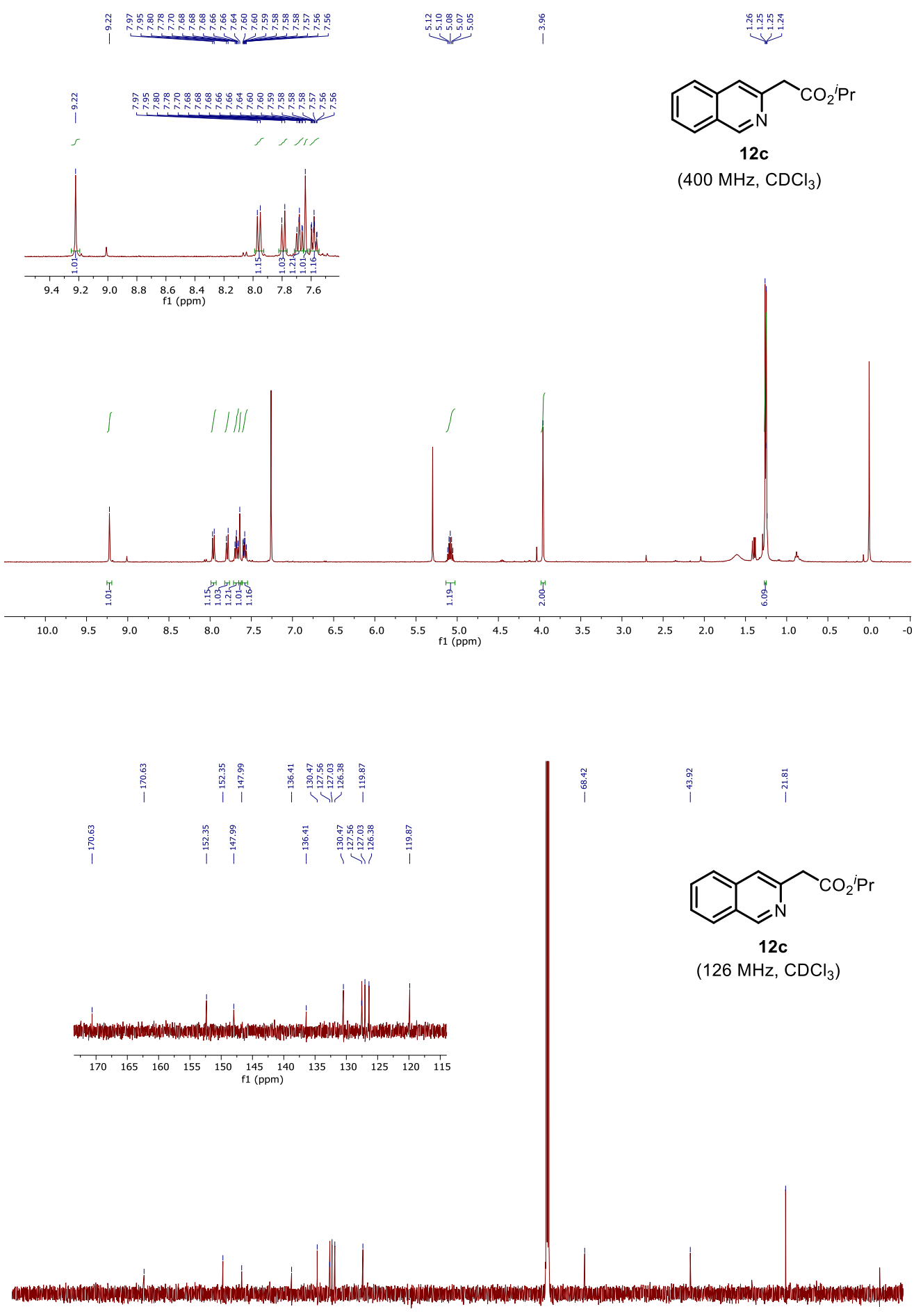

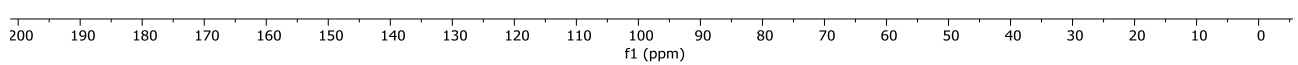




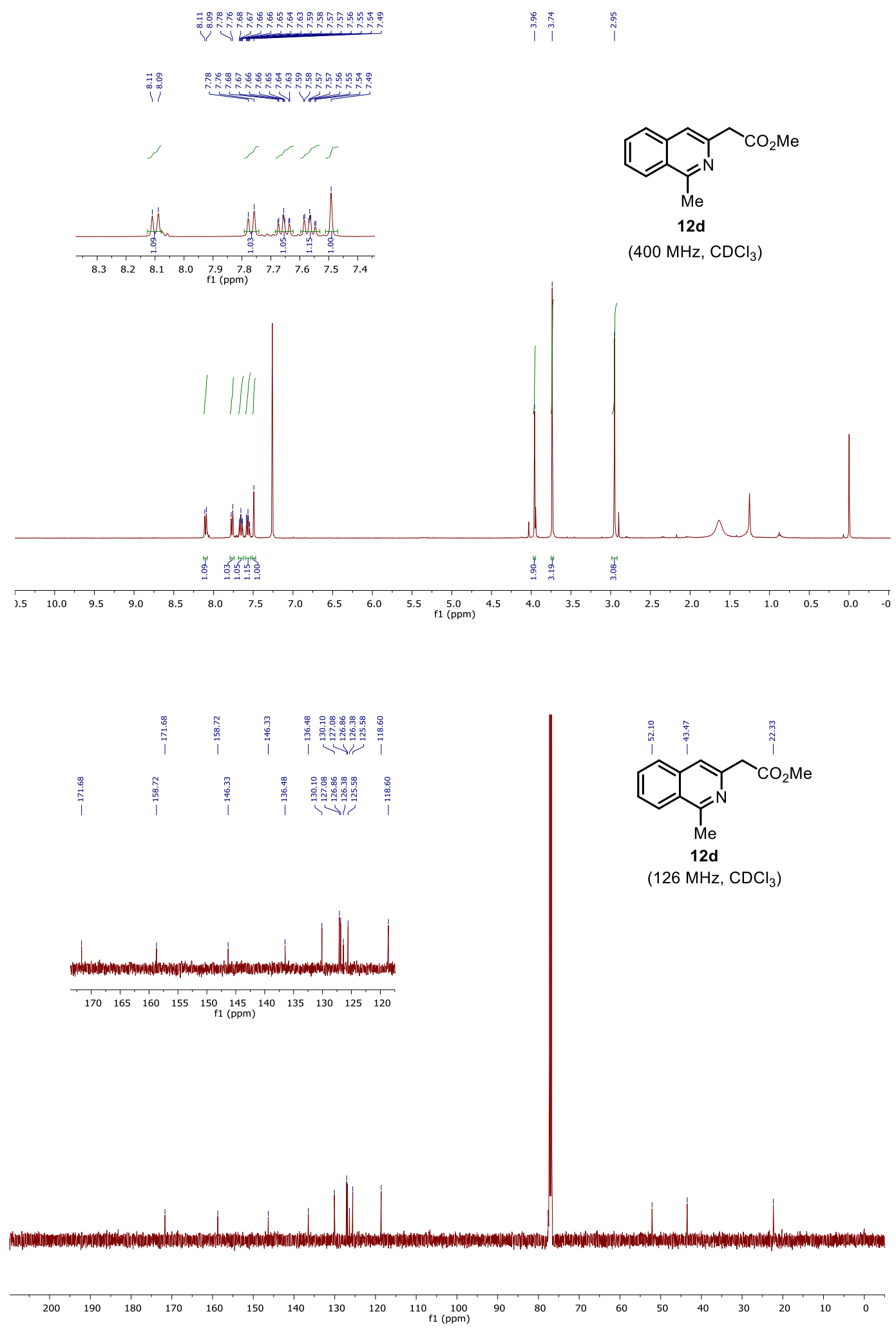

Page S93 of S103 


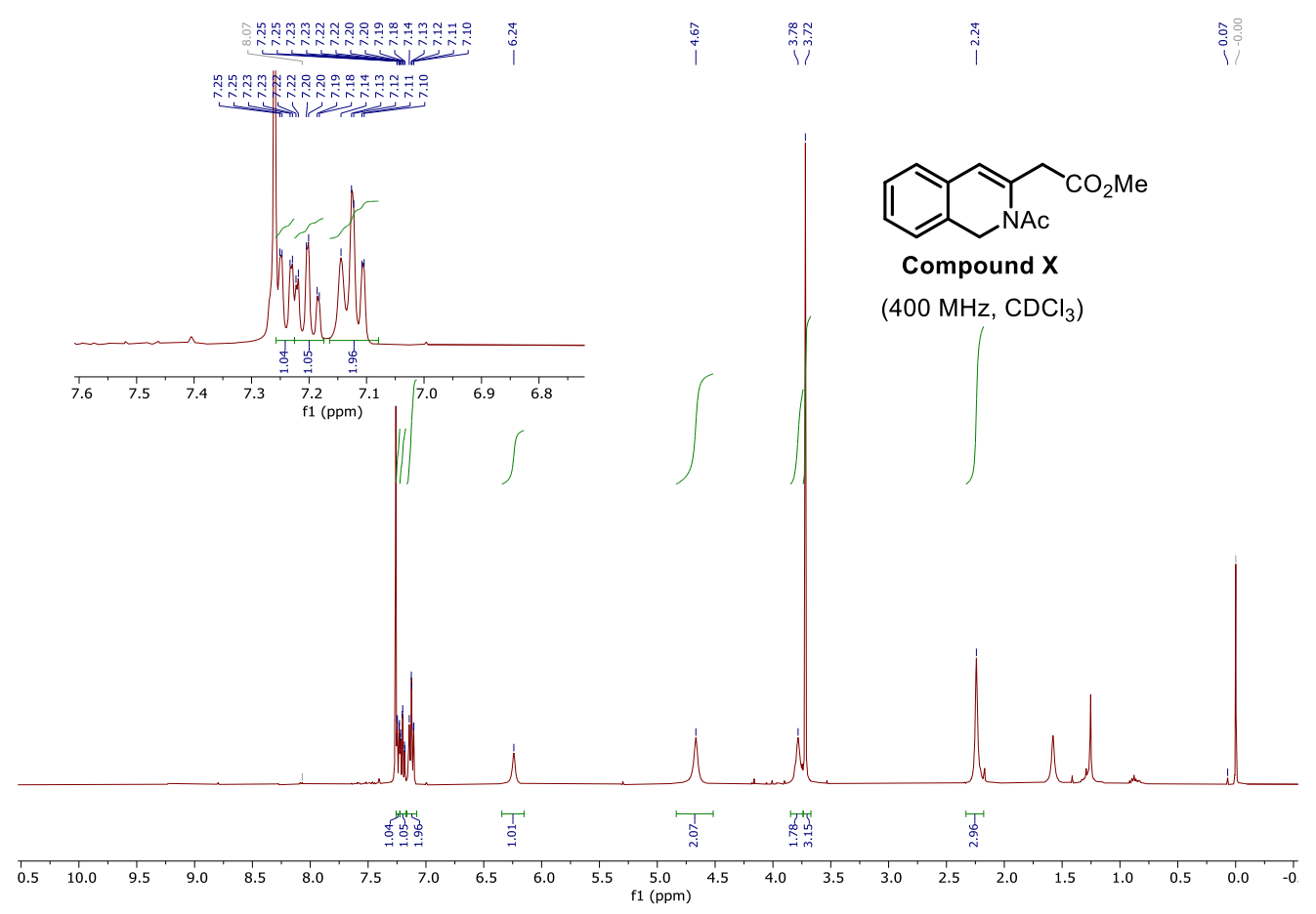

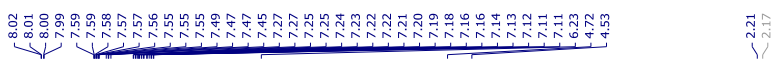
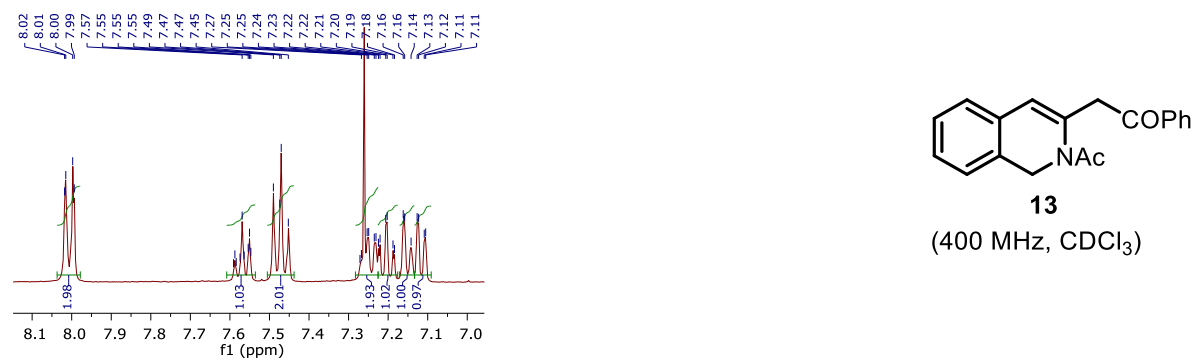

$\left(400 \mathrm{MHz}, \mathrm{CDCl}_{3}\right)$

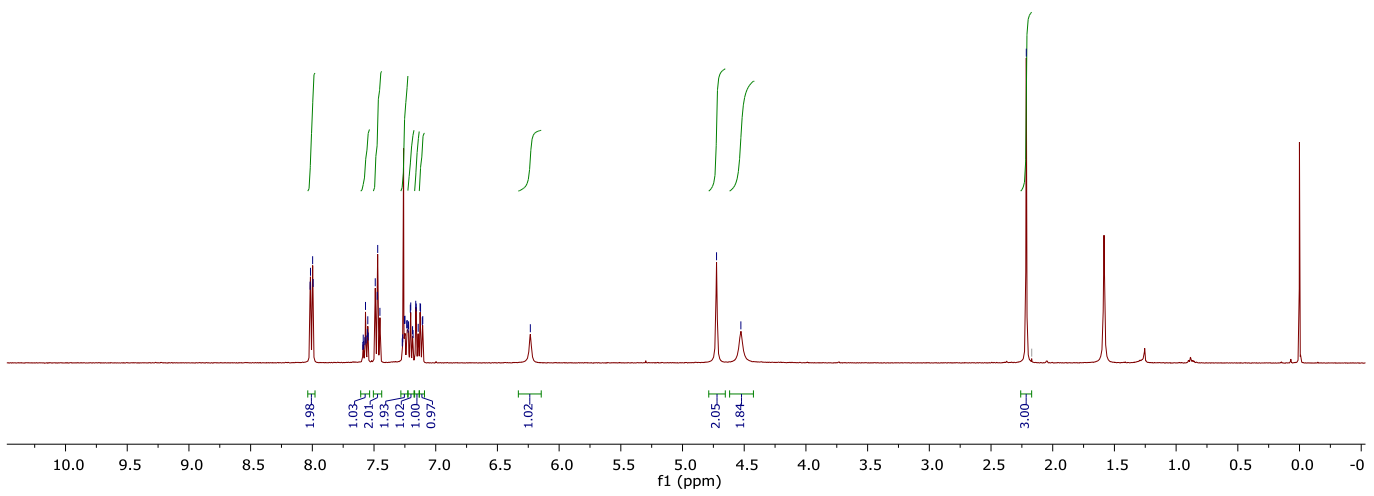

Page S94 of S103 


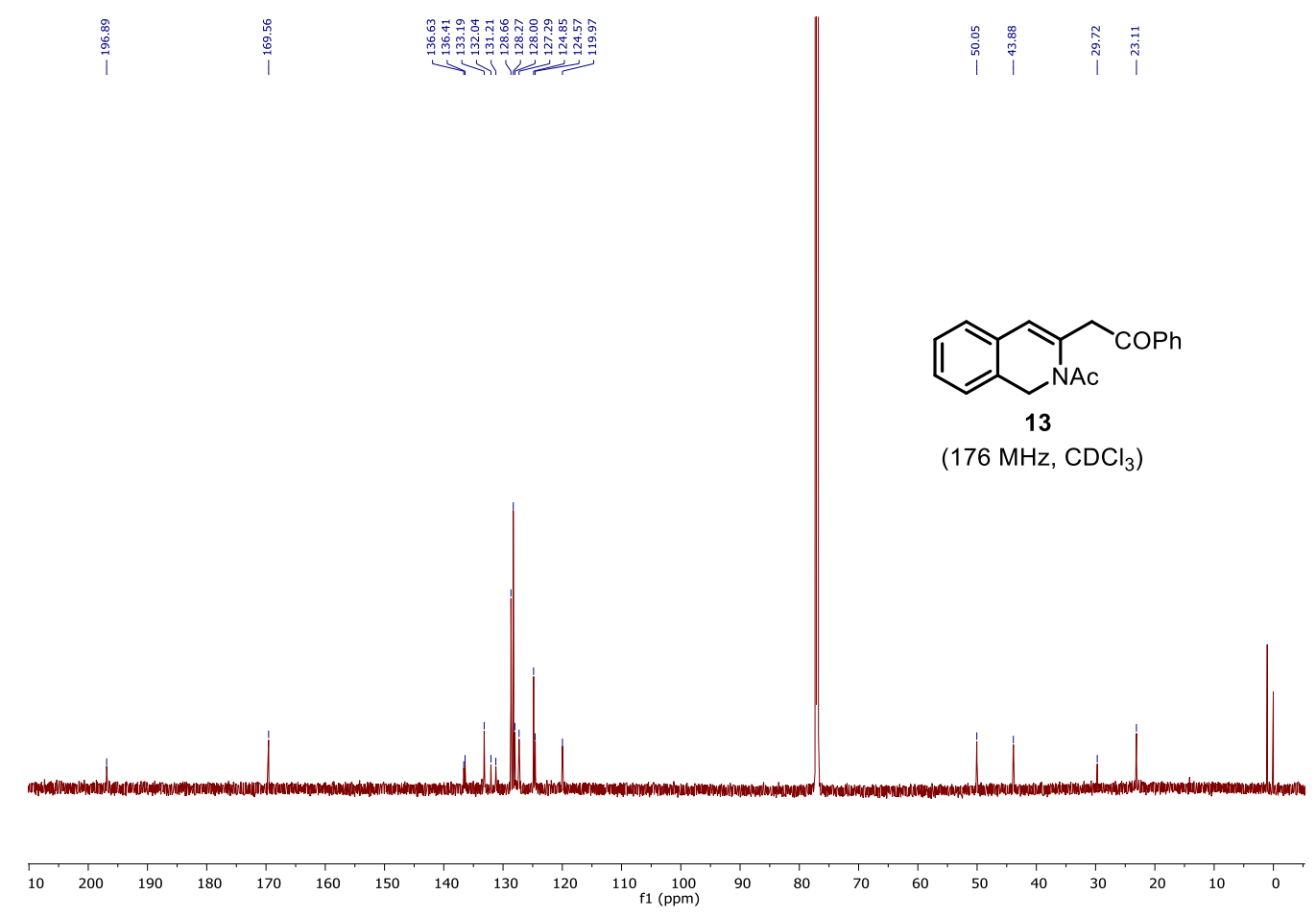

\section{Reversibility Experiment:}

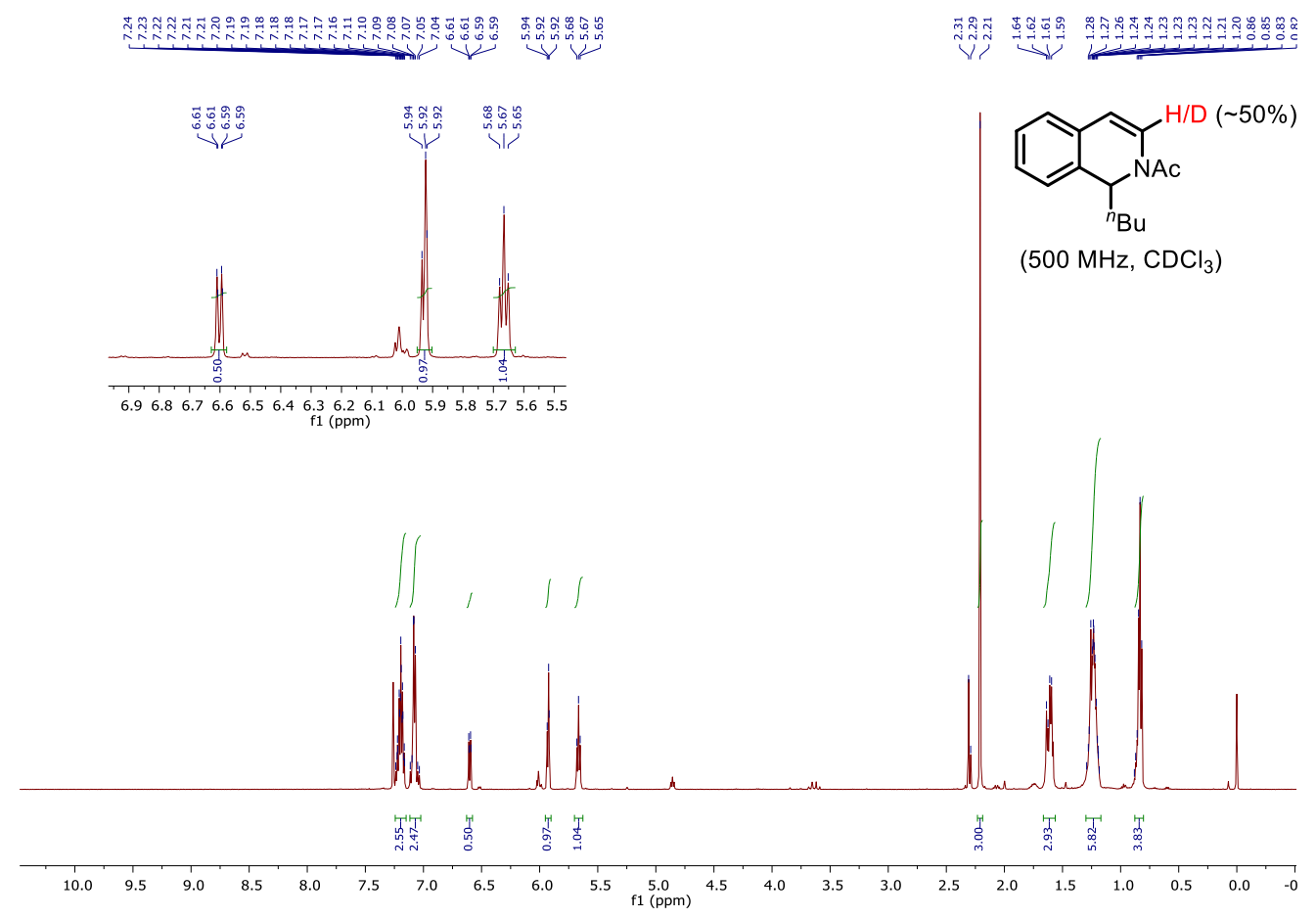

Page S95 of S103 


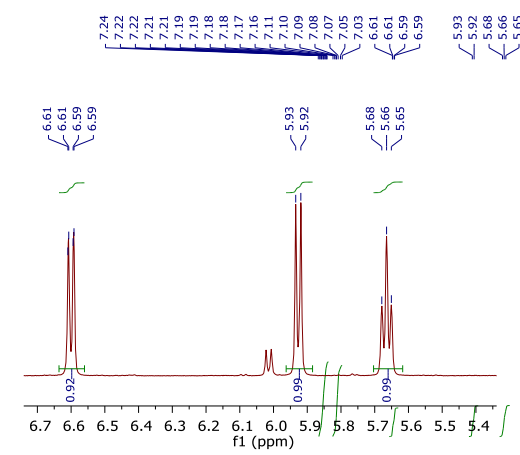

1

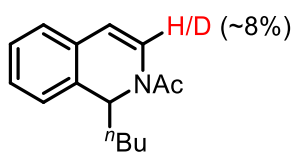

(500 MHz, $\mathrm{CDCl}_{3}$ )
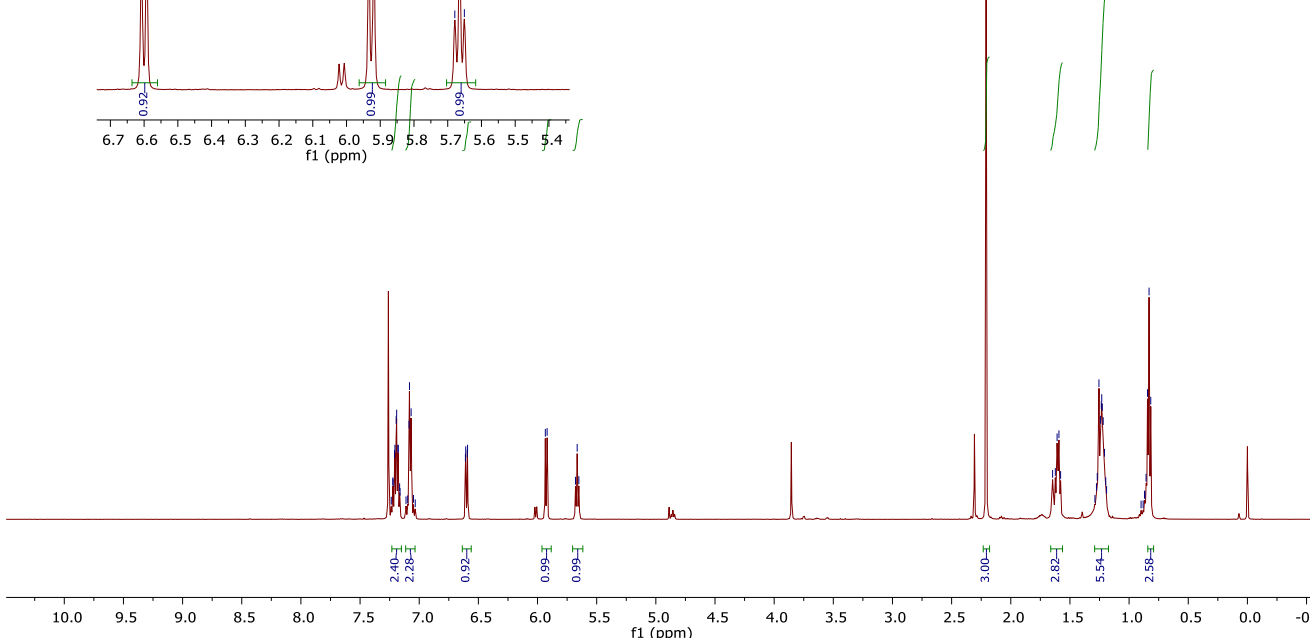
Variable Temperature NMR: in $\mathrm{CDCl}_{3}$

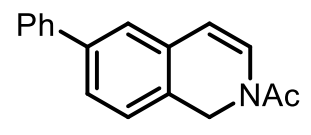

(mixture of rotamers)

Ip

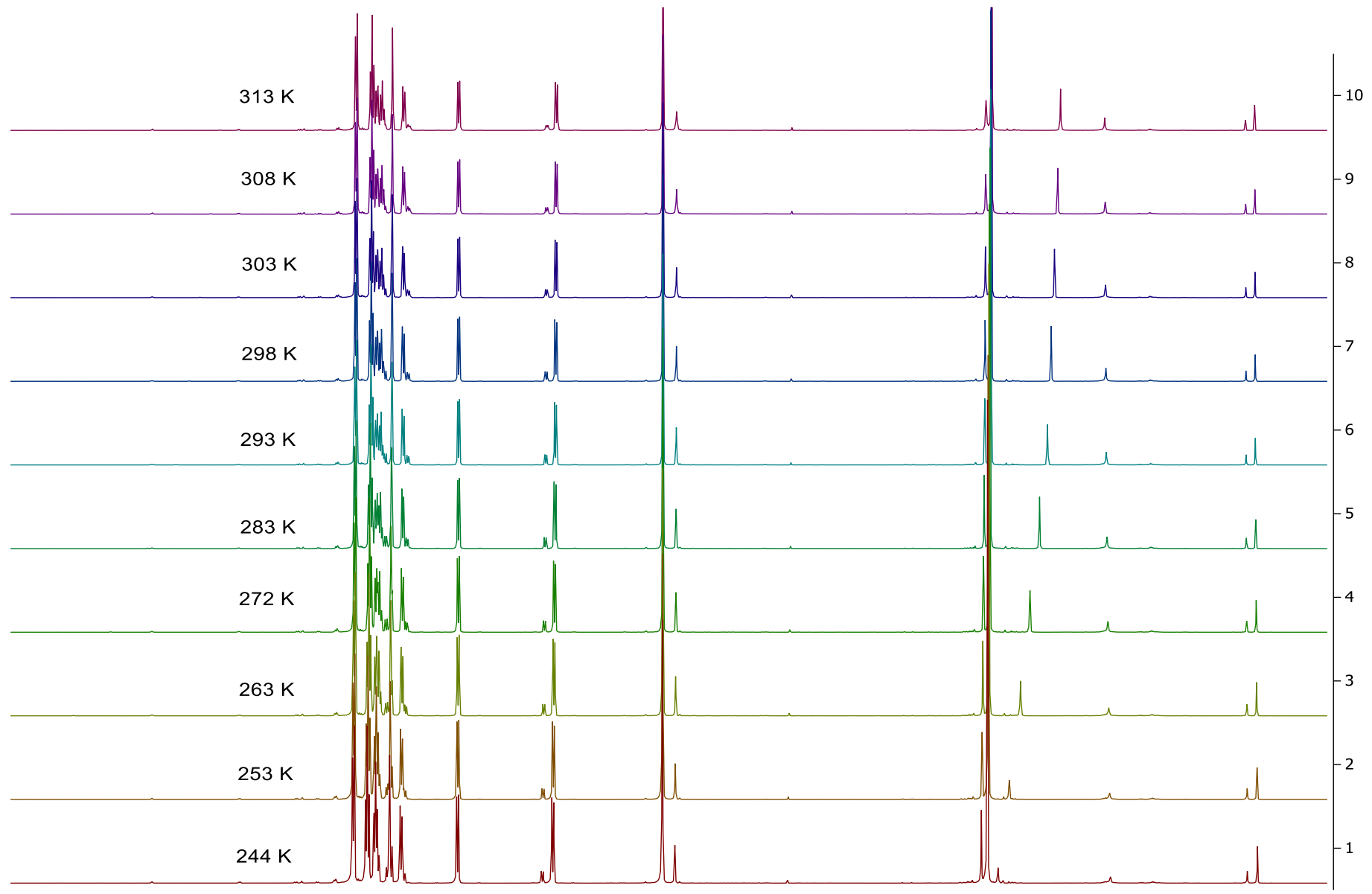

$\begin{array}{llllllllllllllllllllllllll}10.0 & 9.5 & 9.0 & 8.5 & 8.0 & 7.5 & 7.0 & 6.5 & 6.0 & 5.5 & \begin{array}{l}5.0 \\ \text { f }\end{array} & 4.5 & 4.0 & 3.5 & 3.0 & 2.5 & 2.0 & 1.5 & 1.0 & 0.5 & 0.0 & -0.5\end{array}$ 
Variable Temperature NMR: in $\mathrm{CDCl}_{3}$

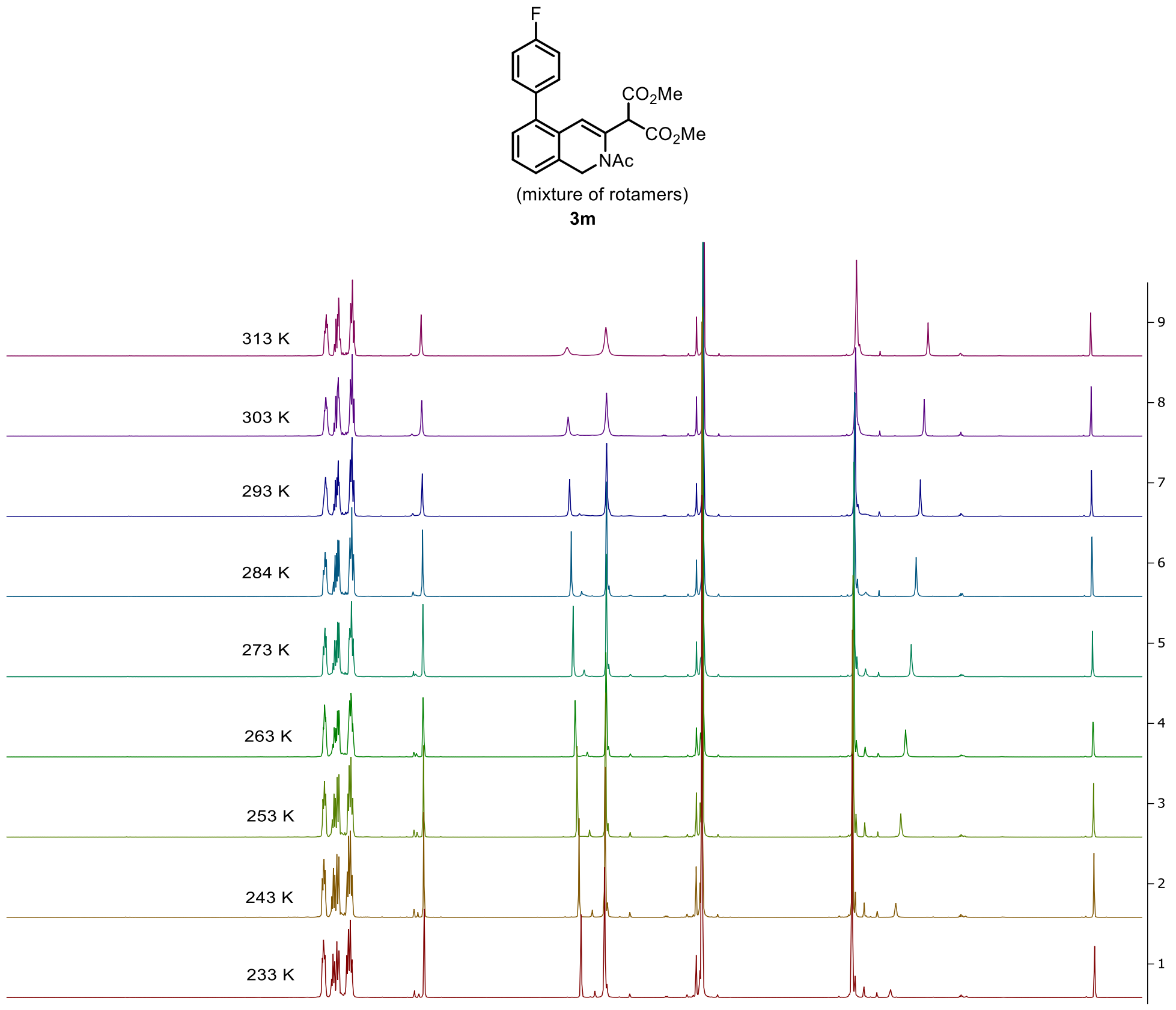

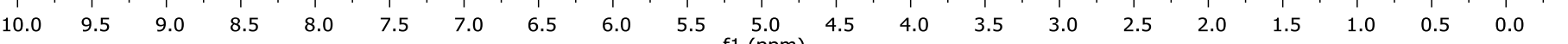




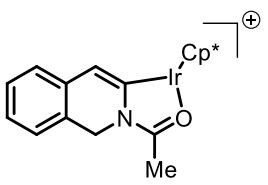

Chemical formula: $\mathrm{C}_{21} \mathrm{H}_{25} \mathrm{NOIr}$

Found [M]: 500.1578

Calcd [M]: 500.1561

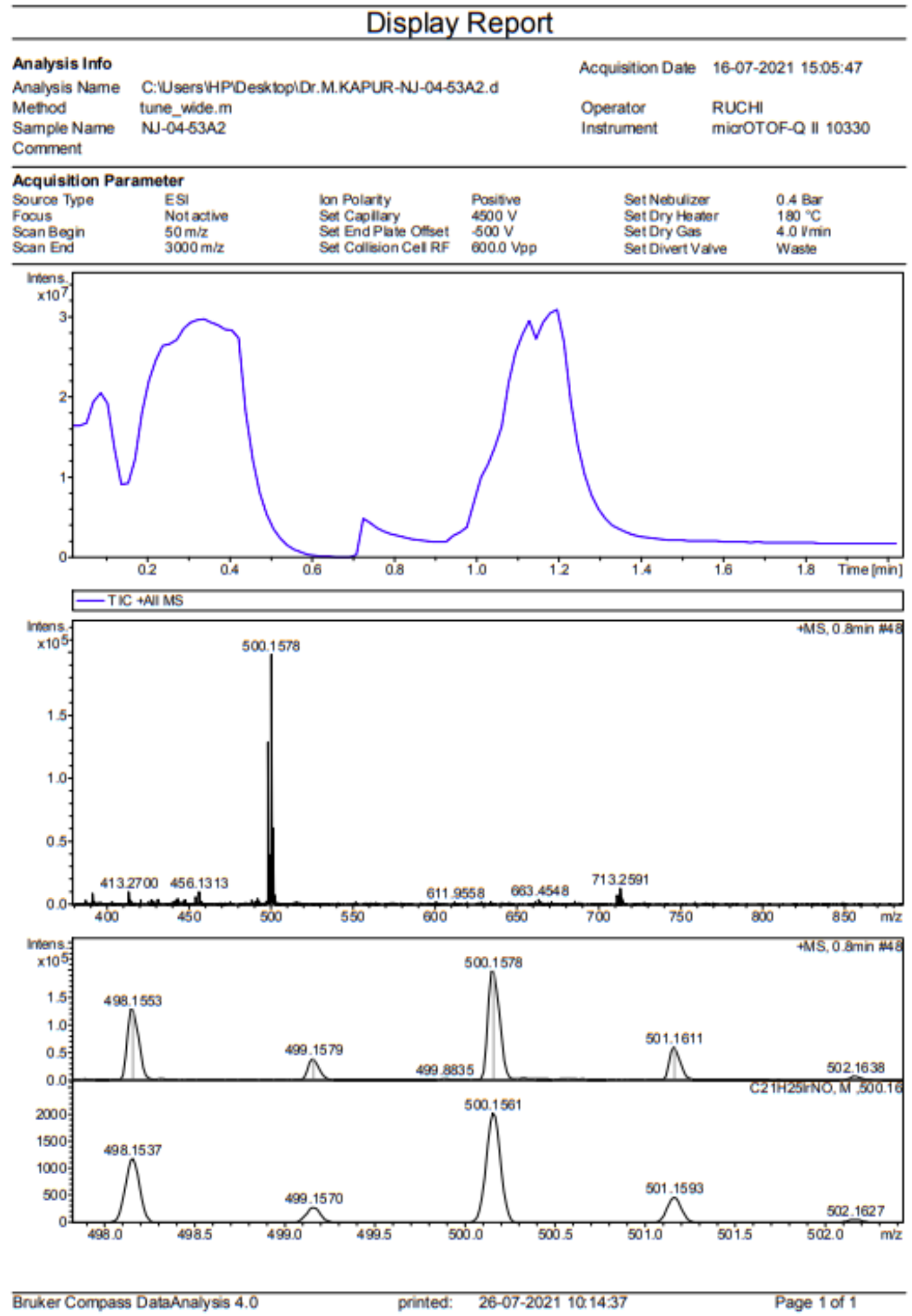




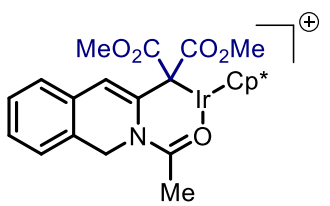

Chemical formula: $\mathrm{C}_{26} \mathrm{H}_{31} \mathrm{NO}_{5} \mathrm{Ir}$

Found [M]: 630.1850

Calcd [M]: 630.1827

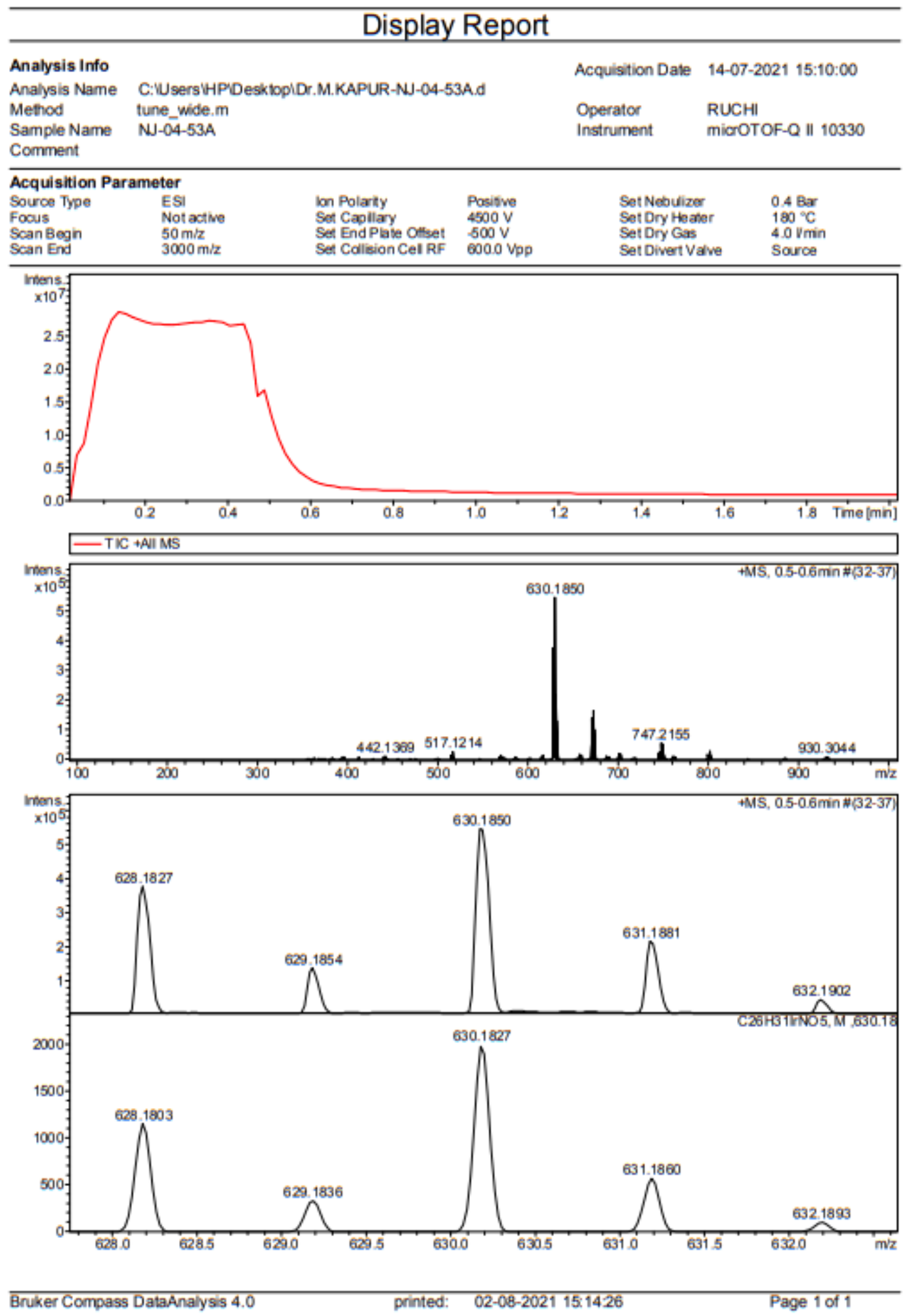




\section{X-ray crystallographic study of compound $7 \mathrm{~b}$ :}

Solvent system for crystal growth- 1:1 (DCM/Hexane); the compound is dissolved in DCM followed by dropwise addition of hexane through the walls of the vial. A perforated cap was placed on the vial. Crystals were observed after 3-4 days.

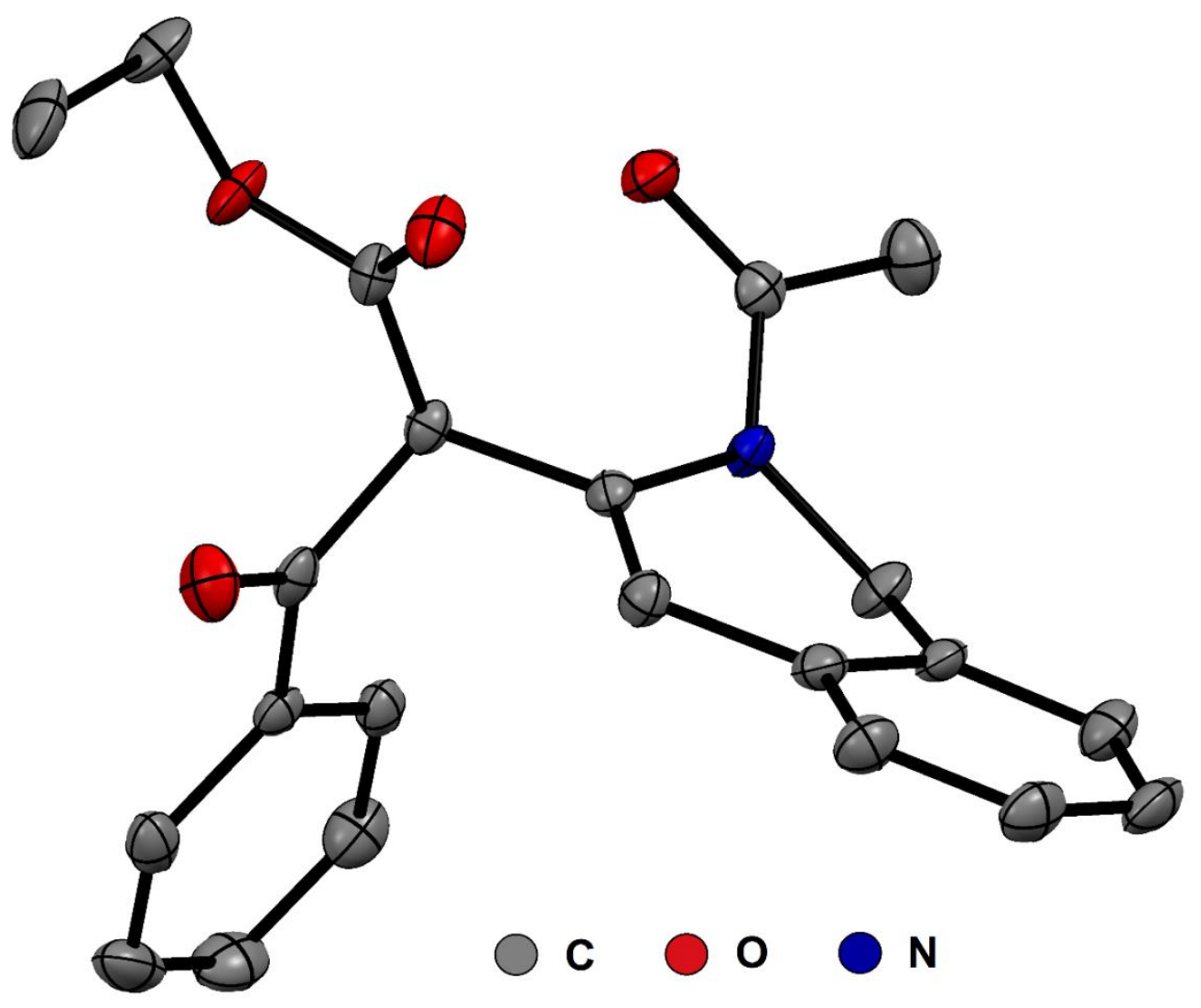

Figure 1. Thermal ellipsoidal plot (50\% probability) for compound PS13124. Hydrogen atoms have been removed for clarity.

Single crystal data of the compound PS13124 was collected on the Bruker D8 VENTURE diffractometer equipped with CMOS type (PHOTON 100) detector using monochromated Mo $\mathrm{K} \alpha$ radiation $(\lambda=0.71073 \AA)$. Unit cell measurement, data collection, integration, scaling and absorption corrections for the crystal were done using Bruker Apex II software ${ }^{[1]}$. Data reduction was done by Bruker SAINT Suite ${ }^{[2]}$. The crystal structure was solved by direct methods using SHELXT $2014^{[3]}$ and refined by the full matrix least squares method using SHELXL $2018{ }^{[4]}$ present in the program suite WinGX (version 2014.1) ${ }^{[5]}$. Absorption correction was applied using SADABS ${ }^{[6]}$. All non-hydrogen atoms were refined anisotropically 
and all hydrogen atoms were positioned geometrically and refined using ariding model with $\mathrm{Uiso}(\mathrm{H})=1.2 \mathrm{Ueq}\left[\mathrm{C}_{\text {aromatic }}, U_{\text {iso }}(\mathrm{H})=1.5 \mathrm{Ueq}\right.$ (methyl groups). ORTEP was generated using Mercury 3.5.1(CCDC) program[7]. Crystallographic and refinement data of the compound was tabulated in Table-1.

Table 1. Crystal data and structure refinement parameters for PS13124.

\begin{tabular}{|c|c|}
\hline Identification code & PS13124 \\
\hline CCDC & 1900717 \\
\hline Empirical formula & $\mathrm{C} 22 \mathrm{H} 21 \mathrm{NO} 4$ \\
\hline Formula weight & 363.40 \\
\hline Temperature & $130(2) \mathrm{K}$ \\
\hline Wavelength & $0.71073 \AA$ \\
\hline Crystal system & Monoclinic \\
\hline Space group & $C 2 / c$ \\
\hline Unit cell dimensions & $\begin{array}{l}a=24.0570(15) \AA \\
b=11.8340(7) \AA \\
c=17.5769(11) \AA \\
\alpha=90^{\circ} \\
\beta=131.290(3)^{\circ} \\
\gamma=90^{\circ}\end{array}$ \\
\hline Volume & $3759.9(4) \AA^{3}$ \\
\hline $\mathrm{Z}$ & 8 \\
\hline Density (calculated) & $1.284 \mathrm{Mg} / \mathrm{m}^{3}$ \\
\hline Absorption coefficient & $0.088 \mathrm{~mm}^{-1}$ \\
\hline $\mathrm{F}(000)$ & 1536 \\
\hline Crystal size & $0.350 \times 0.120 \times 0.050 \mathrm{~mm}^{3}$ \\
\hline Theta range for data collection & 2.253 to $24.998^{\circ}$. \\
\hline Index ranges & $\begin{array}{l}-28<=\mathrm{h}<=27,-14<=\mathrm{k}<=14,- \\
20<=1<=20\end{array}$ \\
\hline Reflections collected & 29921 \\
\hline Independent reflections & $3309[\mathrm{R}(\mathrm{int})=0.1574]$ \\
\hline Completeness to theta $=25.242^{\circ}$ & $99.9 \%$ \\
\hline Absorption correction & Semi-empirical from equivalents \\
\hline Max. and min. transmission & 0.7458 and 0.5696 \\
\hline Refinement method & Full-matrix least-squares on $\mathrm{F}^{2}$ \\
\hline Data / restraints / parameters & $3309 / 174 / 246$ \\
\hline
\end{tabular}




\begin{tabular}{|l|l|}
\hline Goodness-of-fit on $\mathrm{F}^{2}$ & 1.081 \\
\hline Final R indices [I>2sigma(I)] & $\mathrm{R} 1=0.0579, \mathrm{wR} 2=0.1085$ \\
\hline $\mathrm{R}$ indices (all data) & $\mathrm{R} 1=0.1126, \mathrm{wR} 2=0.1233$ \\
\hline Extinction coefficient & $\mathrm{n} / \mathrm{a}$ \\
\hline Largest diff. peak and hole & 0.284 and -0.311 e. $\AA^{-3}$ \\
\hline
\end{tabular}

\section{References:}

1. Apex2, Version 2 User Manual, M86-E01078, Bruker Analytical X-ray Systems Madison, WI, 2006.

2. Siemens, SMART System, Siemens Analytical X-ray Instruments Inc. Madison, MI, 1995.

3. Sheldrick, G. M. Acta Crystallogr. 2015, A71, 3.

4. Sheldrick, G. M. ActaCrystallogr. 2015, C71, 3.

5. Farrugia, L. J. J. Appl. Crystallogr. 2012, 45, 849.

6. Sheldrick, G. M. SADABS; Bruker AXS, Inc.: Madison, WI, 2007.

7. Macrae, C. F.; Bruno, I. J.; Chisholm, J. A.; Edgington, P. R.; McCabe, P.; Pidcock, E.; RodriguezMonge, L.; Taylor, R.; Streek, J.; Wood, P. A. J. Appl. Crystallogr. 2008, 41, 466. 